\title{
COHOMOLOGICAL ARITHMETIC CHOW RINGS
}

\author{
J. I. BURGOS GIL ${ }^{1}$, J. KRAMER ${ }^{2}$ AND U. KÜHN ${ }^{3}$ \\ ${ }^{1}$ Facultad de Matemáticas, Universidad de Barcelona, Gran Vía $3184^{\circ} 1^{a}$, \\ 08007 Barcelona, Spain (burgos@mat.ub.es) \\ ${ }^{2}$ Institut für Mathematik, Humboldt-Universität zu Berlin, \\ Unter den Linden 6, D-10099 Berlin, Germany \\ ${ }^{3}$ Department Mathematik, Universität Hamburg, \\ Bundesstraße 55, D-20146 Hamburg, Germany
}

(Received 24 October 2003; accepted 8 January 2005)

\begin{abstract}
We develop a theory of abstract arithmetic Chow rings, where the role of the fibres at infinity is played by a complex of abelian groups that computes a suitable cohomology theory. As particular cases of this formalism we recover the original arithmetic intersection theory of Gillet and Soulé for projective varieties. We introduce a theory of arithmetic Chow groups, which are covariant with respect to arbitrary proper morphisms, and we develop a theory of arithmetic Chow rings using a complex of differential forms with log-log singularities along a fixed normal crossing divisor. This last theory is suitable for the study of automorphic line bundles. In particular, we generalize the classical Faltings height with respect to logarithmically singular hermitian line bundles to higher dimensional cycles. As an application we compute the Faltings height of Hecke correspondences on a product of modular curves.
\end{abstract}

Keywords: Arakelov geometry; sheaf cohomology; Deligne-Beilinson cohomology; good hermitian metrics

AMS 2000 Mathematics subject classification: Primary 14G40; 14G35; 14C17; 14C30; $11 \mathrm{G} 18$

\section{Contents}

0. Introduction 2

1. Background results on $K$-theory 13

1.1. $K$-theory 14

1.2. $K$-theory as generalized sheaf cohomology 16

1.3. $\lambda$-structure in $K$-theory and absolute cohomology 22

1.4. Chow Groups of regular schemes $\quad 27$

1.5. Gillet cohomology and characteristic classes 33

2. Some topics from homological algebra 40

2.1. $k$-iterated complexes 40

2.2. Relative cohomology groups 46

2.3. Products in relative cohomology 49

2.4. Truncated relative cohomology groups 52

2.5. Products in truncated relative cohomology $\quad 54$ 
3. Green objects 58

$\begin{array}{ll}\text { 3.1. } \mathcal{G} \text {-complexes } & 58\end{array}$

3.2. Definition of Green objects $\quad 62$

3.3. The $*$-product of Green objects 66

$\begin{array}{ll}\text { 3.4. Associativity and commutativity } & 70\end{array}$

3.5. Functorial properties of Green objects 76

4. Abstract arithmetic Chow groups 83

4.1. Arithmetic varieties $\quad 83$

4.2. Arithmetic Chow groups 85

4.3. Arithmetic intersection pairing $\quad 87$

4.4. Inverse images $\quad 91$

4.5. Proper push-forward $\quad 93$

5. Deligne-Beilinson cohomology as a Gillet cohomology $\quad 95$

5.1. Review of Deligne-Beilinson cohomology $\quad 95$

5.2. Review of Deligne algebras $\quad 97$

5.3. A Gillet complex: the Deligne algebra $\mathcal{D}_{\log } \quad 102$

5.4. Deligne-Beilinson homology of proper smooth varieties 106

5.5. Deligne-Beilinson homology of arbitrary varieties 109

5.6. Classes of cycles and line bundles 116

$\begin{array}{ll}\text { 5.7. Real varieties } & 119\end{array}$

6. Examples of $\mathcal{D}_{\text {log-arithmetic Chow groups }} 121$

6.1. Contravariant $\mathcal{D}_{\text {log-arithmetic }}$ Chow rings 121

6.2. Covariant $\mathcal{D}_{\text {log-arithmetic }}$ Chow groups 130

6.3. Height of a cycle $\quad 135$

7. Arithmetic Chow rings with pre-log-log forms 137

$\begin{array}{ll}\text { 7.1. Pre-log-log forms } & 137\end{array}$

7.2. Pre-log forms 140

7.3. Mixed forms 141

7.4. A $\mathcal{D}_{\text {log-complex with pre-log-log forms }} \quad 144$

7.5. Properties of Green objects with values in $\mathcal{D}_{\text {pre }} \quad 145$

7.6. Push-forward of a $*$-product $\quad 149$

7.7. Arithmetic Chow rings with pre-log-log forms 157

7.8. Application to products of modular curves 164

$\begin{array}{ll}\text { References } & 169\end{array}$

\section{Introduction}

\section{Background}

Arakelov geometry was initiated by Arakelov in his paper [1], where he shows that one can compactify a curve defined over the (spectrum of the) ring of integers of a number field by considering Green functions on the associated complex curve. Subsequently, Arakelov's ideas have been successfully refined by Deligne [25] and Faltings [29], and generalized to higher dimensional arithmetic varieties by Gillet and Soulé who introduced arithmetic 
Chow groups for higher codimensional arithmetic cycles in [36]. An important application of this formalism was Vojta's proof of Faltings's theorem, formerly known as Mordell's conjecture (see $[\mathbf{7 4}]$ ).

The use of analytical objects such as Green functions allows various modifications and extensions of the formalism of arithmetic Chow groups, which is adapted to different situations. Among others, we mention Zhang's work [75] on admissible metrized line bundles and Maillot's work [60] taking into account hermitian line bundles whose metrics are no longer smooth but still continuous; Bost's work [9] on $L_{1}^{2}$-Green functions and Moriwaki's work [63] on degenerate Green currents; Kühn's work [58] treating hermitian line bundles on arithmetic surfaces having logarithmically singular metrics, e.g. the line bundle of modular forms equipped with the Petersson metric on a modular curve, and Burgos's work $[\mathbf{1 4}, \mathbf{1 7}]$ on arithmetic Chow groups and Deligne-Beilinson cohomology.

\section{Motivation}

The main motivation of these notes is to extend Kühn's generalized arithmetic intersection pairing on arithmetic surfaces to higher dimensional arithmetic varieties. While Kühn's intersection pairing, which was motivated by preliminary results of Kramer (see [53]), is given in terms of an explicit formula for the arithmetic intersection number of two divisors (in the spirit of Arakelov), the development of a corresponding higher dimensional theory needs to be approached in a more abstract way. Moreover, we will extend this theory, not only to line bundles with singular metrics, but also to higher rank vector bundles such as the Hodge bundle that appear when considering fibrations of semi-abelian varieties. The study of higher rank vector bundles will be the subject of the forthcoming paper $[\mathbf{1 9}]$.

\section{Arithmetic intersection theory}

An arithmetic ring $\left(A, \Sigma, F_{\infty}\right)$ is a triple consisting of an excellent regular noetherian integral domain $A$, a finite non-empty set $\Sigma$ of monomorphisms $\sigma: A \rightarrow \mathbb{C}$, and an antilinear involution $F_{\infty}: \mathbb{C}^{\Sigma} \rightarrow \mathbb{C}^{\Sigma}$ of $\mathbb{C}$-algebras. For simplicity, we will forget about the antilinear involution $F_{\infty}$ in this introduction. Then, an arithmetic variety is a flat, regular scheme $X$ over $S=\operatorname{Spec}(A)$ together with a complex analytic space $X_{\infty}$ obtained from $X$ by means of $\Sigma ; S$ will be called the base scheme. Intuitively, the elements of $\Sigma$ are the points at infinity of $S$ providing a 'compactification' of $S$, and the analytic space $X_{\infty}$ is the fibre at these points at infinity or archimedean fibre of $X$. An arithmetic intersection theory will involve three main ingredients: first, a geometric intersection theory over the scheme $X$, the geometric part; second, a 'refined' intersection theory over $X_{\infty}$, the analytic part; and finally an interface relating the geometric and the analytic part. The main theme of this paper is to study the second and third of these ingredients, and we will rely on existing geometric intersection theories.

\section{Geometric intersection theory}

It might be useful to review, at this point, the geometric intersection theories we have at our disposal. 
The first of these theories is based on the moving lemma to reduce the intersection product of two algebraic cycles to the case of proper intersection. In order to be able to apply the moving lemma we need the scheme $X$ under consideration to be quasiprojective and the arithmetic ring $A$ to be a field. Since we are interested in more general arithmetic rings, we cannot use this method.

The second approach is the deformation to the normal cone technique due to Fulton and MacPherson (see [33]). This method is much more general, since the scheme $X$ need not be quasi-projective and, moreover, it can also be applied to the case in which the base scheme is the spectrum of a Dedekind domain. But in this case $X$ needs to be not only regular, but smooth over $S$. Since most interesting arithmetic varieties are not smooth over the base scheme, this method is not general enough for our purposes. Note however that, in the case in which $X$ is smooth over $S$, this method provides an intersection pairing which is defined without tensoring with $\mathbb{Q}$. We should also note that, in contrast to the case of an intersection product, this method can be used to define the inverse image morphism for a morphism between regular schemes of finite type over the spectrum of a regular, noetherian ring without the assumption of smoothness. The lack of a general theorem of resolution of singularities prevents us from obtaining an intersection product from this inverse image morphism.

However, as a third approach, one can use de Jong's theorem on alterations [22] to reduce the intersection of algebraic cycles to the inverse image between regular schemes, and then apply the deformation to the normal cone technique. Nevertheless, these alterations are finite morphisms whose degree is larger that one, in general. Therefore, this method yields an intersection product with rational coefficients.

The last general approach that we will mention (and the one introduced originally by Gillet and Soulé) is to use the isomorphism between $K$-theory and Chow groups to transfer the ring structure of $K$-theory to the Chow groups. This method is valid for any regular, noetherian scheme $X$ of finite Krull dimension. The main drawback of this method is that the isomorphism between $K$-theory and Chow groups is only true in general after tensoring with $\mathbb{Q}$. Therefore, one also obtains a rational valued intersection product.

Finally, we note that the intersection product with a divisor on a regular scheme can be defined directly using a simple version of the moving lemma for divisors (see [36, 4.2.3.2]), which holds in complete generality.

The $K$-theoretical method and the alteration method are the most general of the above methods. Since the $K$-theoretical approach was the one used by Gillet and Soulé in $[\mathbf{3 6}]$ and, conceptually, fits very well with the cohomological approach we will be using for the analytical part, we also use it as the geometric part of our arithmetic intersection theory. But, since the geometric and the analytic part of our arithmetic intersection theory are isolated and related by a clear interface, we hope that the reader will have no difficulty in using any other of these geometric methods when applicable.

\section{Green currents and Green forms}

We now discuss the refined intersection theories which have been used as the analytic part of a higher dimensional arithmetic intersection theory. 
The first one is the notion of a Green current introduced in [36]. Let $y$ be a $p$ codimensional cycle on $X$. A Green current for $y$ is a class of currents

$$
g_{y} \in \widetilde{D}_{X_{\infty}}^{p-1, p-1}=D_{X_{\infty}}^{p-1, p-1} /\left(\partial D_{X_{\infty}}^{p-2, p-1}+\bar{\partial} D_{X_{\infty}}^{p-1, p-2}\right)
$$

satisfying the residue equation

$$
\mathrm{dd}^{\mathrm{c}} g_{y}+\delta_{y}=\omega_{y}
$$

where $\delta_{y}$ is the current given by integration along the cycle $y_{\infty}$ and $\omega_{y}$ is a smooth form uniquely determined by $(0.0)$.

If $y$ and $z$ are two cycles intersecting properly (i.e. with the appropriate codimension) in $X_{\infty}$, the product of two Green currents, called the star-product or $*$-product, is given by the formula

$$
g_{y} * g_{z}=g_{y} \wedge \delta_{z}+\omega_{y} \wedge g_{z}
$$

It is a Green current for the intersection cycle $y \cdot z$. This approach has many analytical difficulties. For instance, some care has to be taken to define $g_{y} \wedge \delta_{z}$. Moreover, this product is not obviously associative and commutative, and the proof that this is indeed the case, is not trivial.

As we have already mentioned this approach has been generalized in several directions. Typically, these generalizations allow the presence of certain singularities for the differential form $\omega_{y}$. But usually only the case when $X_{\infty}$ is of dimension one is treated (see $[\mathbf{9}, \mathbf{5 8}]$ ), or one does not obtain a full ring structure for the generalized arithmetic Chow rings (see $[60]$ ).

There are other proposals for the definition of the product of Green currents. For instance, Harris and Wang [41] have given a definition of the star-product of two Green currents for non-properly intersecting cycles that depends on a deformation of one of the cycles, and Dan [20] has given a definition of the star-product using meromorphic continuation of certain zeta functions.

In [17] Burgos introduced a new definition of Green forms along the following lines. To every complex algebraic manifold $X$ (not necessarily compact), there is associated a graded complex $\mathcal{D}_{\log }^{*}(X, *)$, which consists of smooth forms on $X$ with logarithmic singularities at infinity. For instance, if $X$ is proper, then

$$
\begin{aligned}
\mathcal{D}_{\log }^{2 p}(X, p) & =E^{p, p}(X) \cap(2 \pi \mathrm{i})^{p} E_{\mathbb{R}}^{2 p}(X), \\
\mathcal{D}_{\log }^{2 p-1}(X, p) & =E^{p-1, p-1}(X) \cap(2 \pi \mathrm{i})^{p-1} E_{\mathbb{R}}^{2 p-2}(X),
\end{aligned}
$$

where $E^{p, p}(X)$ is the space of smooth complex valued differential forms of type $(p, p)$ and $E_{\mathbb{R}}^{2 p}(X)$ is the space of smooth real valued differential forms. The boundary morphism

$$
\mathrm{d}_{\mathcal{D}}: \mathcal{D}_{\log }^{2 p-1}(X, p) \rightarrow \mathcal{D}_{\log }^{2 p}(X, p)
$$

is given by $\mathrm{d}_{\mathcal{D}} \eta=-2 \partial \bar{\partial} \eta$. Observe that, up to a normalization factor, this is the same differential operator as the one that appears in the residue equation (0.0).

The complex $\mathcal{D}_{\log }^{*}(X, *)$ computes the real Deligne-Beilinson cohomology of $X$, which is denoted by $H_{\mathcal{D}}^{*}(X, \mathbb{R}(p))$. If $Y$ is a closed subset of $X$, then the real Deligne-Beilinson 
cohomology of $X$ with supports on $Y$, denoted by $H_{\mathcal{D}, Y}^{*}(X, \mathbb{R}(p))$, is the cohomology of the simple complex associated to the morphism of complexes

$$
\mathcal{D}_{\log }^{*}(X, *) \rightarrow \mathcal{D}_{\log }^{*}(X \backslash Y, *) .
$$

Every $p$-codimensional algebraic cycle $y$ with support $Y$ defines a cohomology class $\operatorname{cl}(y) \in H_{\mathcal{D}, Y}^{2 p}(X, \mathbb{R}(p))$. Moreover, if $W \subseteq X$ is a closed subvariety of codimension $p-1$ and $f \in K(W)^{*}$, then $f$ defines a class

$$
\operatorname{cl}(f) \in H_{\mathcal{D}}^{2 p-1}(X \backslash \operatorname{div}(f), \mathbb{R}(p)) .
$$

These classes satisfy the compatibility condition

$$
\delta(\operatorname{cl}(f))=\operatorname{cl}(\operatorname{div}(f)),
$$

where

$$
\delta: H_{\mathcal{D}}^{2 p-1}(X \backslash \operatorname{div}(f), \mathbb{R}(p)) \rightarrow H_{\mathcal{D}, \operatorname{div}(f)}^{2 p}(X, \mathbb{R}(p))
$$

is the connecting homomorphism.

In this setting a Green form for a $p$-codimensional algebraic cycle $y$ is a representative of the class $\mathrm{cl}(y)$. More explicitly, we write

$$
\begin{aligned}
Z \mathcal{D}_{\log }^{*}(X, *) & =\operatorname{Ker}\left(\mathrm{d}_{\mathcal{D}}\right), \\
\widetilde{\mathcal{D}}_{\log }^{*}(X, *) & =\mathcal{D}_{\log }^{*}(X, *) / \operatorname{Im}\left(\mathrm{d}_{\mathcal{D}}\right) .
\end{aligned}
$$

The space of Green forms associated to a $p$-codimensional cycle $y$ with support $Y$ is then given by

$$
G E(y)=\left\{\left(\omega_{y}, \widetilde{g}_{y}\right) \in \mathrm{ZD}_{\log }^{2 p}(X, p) \oplus \widetilde{\mathcal{D}}_{\log }^{2 p-1}(X \backslash Y, p) \mid \begin{array}{c}
\mathrm{d}_{\mathcal{D}} \widetilde{g}_{y}=\omega_{y} \\
\operatorname{cl}\left(\left(\omega_{y}, \widetilde{g}_{y}\right)\right)=\operatorname{cl}(y)
\end{array}\right\} .
$$

The star-product of Green forms is now simply the cup product in cohomology with supports. With this approach, the proof of the associativity and commutativity of the star-product is straightforward and completely formal.

In $[\mathbf{1 6}]$ and $[\mathbf{1 7}]$ it is proven that, when $X$ is projective, the arithmetic Chow groups obtained by this method agree with the ones obtained by the method of Gillet and Soulé. It is interesting to note that all the analytical complexities appearing in the proof of the associativity and commutativity of the star-product in [36] are needed to prove the compatibility of the two definitions.

In contrast, in the quasi-projective case, the groups obtained by this new method have better Hodge theoretical properties. For instance, they possess a certain homotopy invariance with respect to vector bundles. Another advantage of this new definition is that it is very easy to make variants adapted to new problems just by changing the complex $\mathcal{D}_{\log }$.

We should stress here that a Green form associated to a cycle is a representative of the cohomology class of the cycle with support in the same cycle. In order for the starproduct of two Green forms to be a Green form for the intersection cycle, we need the 
cycles to intersect properly in $X_{\infty}$. Therefore, as was the case in [36], the arithmetic intersection product relies on the moving lemma for complex varieties. In particular, this implies that our varieties should be at least geometrically quasi-projective.

In the preprint [45], Hu uses the homotopy invariance and the flexibility of the definition of Green forms to give a new definition of the inverse image morphism of arithmetic Chow groups for a regular closed immersion by means of Fulton's deformation to the normal cone technique. The main result of this paper is the construction of a specialization morphism for Green forms. Combining this technique with de Jong's result on alterations mentioned above, one can define the arithmetic intersection pairing without using any moving lemma, thereby removing the hypothesis of quasi-projectivity.

\section{The interface between the geometric and the analytic part}

In the definition of arithmetic Chow groups by means of Green currents, this interface is implemented by very concrete objects. Namely, to any $p$-codimensional algebraic cycle $y$, one assigns the current 'integration along the cycle $y_{\infty}$ ', denoted by $\delta_{y}$, and to every rational function $f$, one associates the current $-\log \left|f_{\infty}\right|^{2}$. Compared with to the approach of Green currents, this interface is more abstract. It is implemented by the theory of characteristic classes for cycles and rational functions. The two approaches are compatible because in the appropriate complexes, the currents $\delta_{y}$ and $-\log \left|f_{\infty}\right|^{2}$ represent the cohomology class of $y_{\infty}$ and $f_{\infty}$, respectively.

\section{Abstract arithmetic Chow groups}

Recall that our main motivation is to extend Kühn's generalized arithmetic intersection pairing on arithmetic surfaces to higher dimensional arithmetic varieties. In order to accomplish this goal we will use the flexibility of the Green form approach, changing the complex $\mathcal{D}_{\log }$ by a complex of differential forms with certain mild singularities along a fixed subvariety. Nevertheless, the arithmetic Chow groups that we will define in this way, and their properties, depend strongly on the actual complex used. And there is no one best choice for this complex of singular differential forms. For instance, in this paper we introduce the complex of pre-log-log differential forms which, although it does not have all the cohomological properties one would expect, is enough to define an arithmetic intersection pairing and, in particular, the height with respect to log singular hermitian line bundles. On the other hand, in the paper [19], we introduce the complex of loglog singular differential forms. This complex has the expected cohomological properties, but is slightly more difficult to handle because one has to bound all the derivatives of the functions involved. Moreover, as we mentioned previously, in the literature there are several other variants of arithmetic Chow groups with singular differential forms.

In addition, if one uses completely different kinds of complexes, one can obtain arithmetic Chow groups with new properties. For instance, in his $\mathrm{PhD}$ thesis [14], Burgos constructed a fully covariant version of the arithmetic Chow groups by choosing a complex of currents instead of the complex of smooth differential forms. Similar arithmetic Chow groups were introduced independently by Moriwaki in [63]. 
In another direction, one may consider the following example. Let $p_{i}, i=1, \ldots, 4$, be four different points in $\mathbb{P}_{\mathbb{Q}}^{1}$. Then, the archimedean component of the height pairing of $p_{1}-p_{2}$ and $p_{3}-p_{4}$ is essentially given by the logarithm of the norm of the cross ratio of the four points. Thus, this height pairing has a canonical lifting from $\mathbb{R}$ to $\mathbb{C}^{*}$, namely the cross ratio of the four points. This suggests that one can define a finer version of arithmetic Chow groups, where the role of real Deligne-Beilinson cohomology is played by integral Deligne-Beilinson cohomology.

Even more, one can think of an adelic version of the arithmetic Chow groups, similar to the one introduced in [7], but where each geometric fibre is substituted by a suitable complex that computes a certain cohomology theory, or a theory, where only certain geometric fibres are substituted by a cohomological complex.

All these considerations lead us to the conclusion that it is worthwhile to develop a formalism of arithmetic Chow groups with respect to an abstract complex and to explore how the properties of the complex are reflected by the properties of the arithmetic Chow groups. In this way, different variants of arithmetic Chow groups can be obtained as particular cases.

\section{Results}

The main achievement of this paper is the systematic development of the formalism of abstract arithmetic Chow groups in arbitrary dimensions depending on a suitable cohomological complex. Every choice of such a complex gives rise to new types of arithmetic Chow groups with different properties reflected by the complexes under consideration. As special cases we recover Burgos's version of the arithmetic intersection theory developed by Gillet and Soulé in [36], we introduce a theory of arithmetic Chow groups which are covariant with respect to arbitrary proper morphisms, and we develop a theory of arithmetic Chow rings using a complex of differential forms with pre-log-log singularities along a fixed normal crossings divisor. This latter theory is suitable for the study of automorphic line bundles. In particular, we generalize the classical Faltings height with respect to a logarithmically singular hermitian line bundle to higher dimensional cycles. As an application we compute the Faltings height of Hecke correspondences on a product of modular curves.

This formalism of arithmetic Chow groups is an abstraction of [17]. Note however that the passage from the concrete example of [17] to the abstract version presented here is not completely straightforward. Although some constructions such as the definition of truncated cohomology classes and their product are already (at least implicitly) present in $[\mathbf{1 7}]$, others, like the notions of $\mathcal{G}$-complex and of covariant $f$-morphism of complexes, are new.

The basic idea of this paper is that the role of the complex $\mathcal{D}_{\log }$ can be played by any graded complex of sheaves in the Zariski topology $\mathcal{C}$. We only require two properties for this complex. The first is that the hypercohomology of this complex of sheaves always agrees with the cohomology of the complex of global sections. In this way we can represent cohomology classes by concrete elements of this complex. A convenient way to ensure this is to ask the complex to satisfy a Mayer-Vietoris condition. The second 
property we require is that the complex receives characteristic classes from cycles and rational functions (i.e. from $K_{1}$-chains). Typically, in order to ensure the existence of characteristic classes, one imposes a series of axioms to the cohomology. Nevertheless, in many applications it is not convenient to impose too many conditions to the cohomology. Therefore, we use an auxiliary cohomology, given by a graded complex of sheaves $\mathcal{G}$ satisfying the axioms of [34]; we call it a Gillet cohomology. The characteristic classes will be induced by a morphism $\mathfrak{c}_{\mathcal{C}}: \mathcal{G} \rightarrow \mathcal{C}$ in the derived category.

In this abstract setting, instead of Green forms, we will define Green objects that live in a space that we call 'truncated cohomology classes'. These truncated cohomology classes are something between relative cohomology classes and representatives of relative cohomology classes.

Of course, very little can be done with this minimal set of properties. Therefore, we undertake a complete study of how the properties of the complex $\mathcal{C}$ are reflected by the properties of the Green objects and the arithmetic Chow groups. For instance, in order to have an intersection product in the arithmetic Chow groups, we only need the existence of a cup product in the complex $\mathcal{C}$, which is compatible with the cup product of the complex $\mathcal{G}$. This implies that the cup product in $\mathcal{C}$ is compatible with the intersection product of cycles.

We emphasize here that this abstract approach also simplifies many difficulties that appear when working with Green currents. We have already mentioned the proof of the associativity and commutativity of the star-product, but our approach also provides a new and logically independent proof of the well-definedness of the arithmetic intersection product due to Gillet and Soulé. This proof does not rely on the $K_{1}$-chain moving lemma. We point out that in Burgos's preceding work the arguments for proving the welldefinedness of the arithmetic intersection product relied on the corresponding arguments in [36]. We will give a more detailed discussion on how we avoid the $K_{1}$-chain moving lemma in Remark 4.22. We also emphasize that some problems with the $K_{1}$-chain moving lemma have been discussed and successfully solved using completely different techniques by Gubler in [40].

Once the abstract theory is developed, in the subsequent sections, we study particular cases of this construction. For instance, we recover the original arithmetic intersection theory of Gillet and Soulé for projective varieties. We point out that we will show in [19] how to recover these arithmetic Chow groups for quasi-projective varieties as a particular case of our construction. This example agrees with the theory developed in [17].

As a second example we introduce a theory of arithmetic Chow groups which are covariant with respect to arbitrary proper morphisms. This construction was first introduced in [14], and a similar construction can be found in [63].

Furthermore, by choosing for $\mathcal{C}$ a complex of forms satisfying certain growth conditions of log- and log-log-type, we obtain a theory which is compatible with the theories developed by Bost and Kühn in the one-dimensional setting. This latter theory is specifically suited for the study of automorphic line bundles and allows to generalize the classical Faltings height with respect to a logarithmically singular hermitian line bundle to higher dimensional cycles. As an application, we compute the Faltings height of certain Hecke 
correspondences. Note that the same result has been obtained recently by Autissier [3] using the one-dimensional theory.

We point out that Bost's theory can also be seen as a particular case of our abstract setting. Nevertheless, the definition of the corresponding complex $\mathcal{C}$ involves a mixture of $L^{2}$-forms, $L_{1}^{2}$-forms, $L_{-1}^{2}$-currents, forms with logarithmic singularities, and currents; we will not write it explicitly.

It would be interesting to extend the abstract setting of this paper to cover also Hu's deformation to the normal cone technique. This would involve incorporating the specialization functor to the axiomatic system and asking for the existence of a specialization morphism at the level of complexes.

\section{Applications}

The theory developed in this paper is extensively used in the forthcoming paper [13] by Bruinier, Burgos and Kühn, where explicit calculations for the arithmetic self-intersection number of the line bundle of modular forms and the Faltings height of HirzebruchZagier cycles on Hilbert modular surfaces are carried out. Further calculations in this direction for other naturally metrized automorphic line bundles have been made in [12]. In his forthcoming thesis Freixas-Montplet will prove finiteness results for the height with respect to such naturally metrized line bundles, which generalize Faltings's original result for points to cycles of higher dimensions. In the sequel [19] of this paper, we will show that our abstract arithmetic Chow groups attached to the complex of forms having suitable growth conditions of log- and log-log-type combined with the work [18] allow us to construct arithmetic characteristic classes for vector bundles equipped with hermitian metrics, which are logarithmically singular along a divisor with normal crossings. These arithmetic characteristic classes give rise to operations on the arithmetic Chow groups, even for non-regular arithmetic varieties. In addition, we show in [19] that automorphic vector bundles equipped with the natural invariant metric (Petersson metric) on Shimura varieties of non-compact type are hermitian vector bundles of the type considered above.

The framework of our arithmetic Chow groups attached to forms having certain logand log-log-type singularities is one of the key ingredients in order to formulate various conjectures: in this context, we mention a conjecture of Köhler on arithmetic intersection numbers on the moduli space of principally polarized abelian varieties (see [52]); secondly, we mention a conjecture of Maillot and Roessler on arithmetic Chern numbers associated to fibrations of motives with complex multiplication (see [61]); finally, we emphasize the conjectures of Kudla on Faltings heights and generating series for special cycles on Shimura varieties of orthogonal type (see $[\mathbf{5 5}, \mathbf{5 6}])$.

\section{Outline of the paper}

Let us now give a more detailed outline of the contents of each section.

\section{Section 1}

In the first section we review various results relating $K$-theory, Chow groups and cohomology theories satisfying Gillet's axioms [34]; these facts will be needed in the 
sequel. For our purposes, the main interest in $K$-theory is that it provides a method to define an intersection product and an inverse image for algebraic cycles on regular, separated schemes of finite type over a base scheme, which is regular, separated, noetherian, and of finite Krull dimension. Most of the results needed from $K$-theory deal only with the groups $K_{0}$ and $K_{1}$, and can be found in the first chapter of [71]. The main exception to this is Proposition 1.16; its translation into Chow theory and cohomology theory, namely Theorem 1.34 and Corollary 1.35, is of crucial importance in the fourth section in the course of the proof of the well-definedness of the intersection product of arithmetic cycles. There is very little new in this section and its main purpose is to gather together all the needed results, some of which are difficult to find explicitly in the literature.

\section{Section 2}

The second section is devoted to a systematic study of relative cohomology groups $H^{*}(A, B)$ attached to a morphism $f: A \rightarrow B$ of abstract complexes of abelian groups and their product structure based on a product structure of the complexes under consideration. Furthermore, we study truncated relative cohomology groups $\widehat{H}^{*}(A, B)$ associated to the above data together with their product structure, which is the basis of the definition of the $*$-product in the third section. We have also included a discussion of the signs appearing when considering multidimensional complexes, complexes of complexes, and products between them. This section is an extended and much more detailed version of the corresponding chapter of $[\mathbf{1 7}]$.

\section{Section 3}

The aim of the third section is to develop an abstract theory of Green objects as elements of a suitable truncated cohomology theory. The main property for such a cohomology theory is that it receives characteristic classes from $K$-theory, at least from $K_{0}$ and $K_{1}$, and that it satisfies some additional natural properties. More precisely, we fix a Gillet complex $\mathcal{G}=\mathcal{G}^{*}(*)$ over the site of regular schemes $X$ of finite type over a field $k$. A graded complex $\mathcal{C}=\mathcal{C}^{*}(*)$ of sheaves of abelian groups together with a morphism $\mathfrak{c}_{\mathcal{C}}: \mathcal{G} \rightarrow \mathcal{C}$ in the derived category will be called a $\mathcal{G}$-complex over $X$. A Green object for a $p$-codimensional cycle $y$ on $X$ with values in $\mathcal{C}$ is then given by an element

$$
\mathfrak{g}_{y} \in \widehat{H}^{2 p}(\mathcal{C}(X, p), \mathcal{C}(U, p))
$$

such that the class of $\mathfrak{g}_{y}$ equals the class of the cycle $y$ in the relative cohomology group $H^{2 p}(\mathcal{C}(X, p), \mathcal{C}(U, p))$; here $U$ denotes the complement of the support of $y$ in $X$. After studying the basic properties of such Green objects, we define a *-product for two Green objects using the techniques developed in the second section. We end this section with a proof of the associativity and commutativity of the $*$-product under suitable assumptions on the $\mathcal{G}$-complexes under consideration. The material in this and the next section, although a generalization of the results of $[\mathbf{1 7}]$, is new. 


\section{Section 4}

In the fourth section we introduce generalized arithmetic Chow groups for arithmetic varieties $X$ over an arithmetic ring. The idea behind this definition is that the arithmetic variety $X$ can be 'compactified' by adding the associated complex manifold $X_{\infty}$ or, more precisely, a truncated cohomology theory on $X_{\infty}$ to the picture. More specifically, we proceed as follows: After fixing a Gillet complex $\mathcal{G}$ over the site of schemes over the real numbers, we call the pair $(X, \mathcal{C})$ a $\mathcal{G}$-arithmetic variety, when $X$ is an arithmetic variety and $\mathcal{C}$ a $\mathcal{G}$-complex over the associated real variety $X_{\mathbb{R}}$. The group of $p$-codimensional arithmetic cycles of $(X, \mathcal{C})$ is then given by the set of pairs $\left(y, \mathfrak{g}_{y}\right)$, where $y$ is a $p$ codimensional cycle on $X$ and $\mathfrak{g}_{y}$ is a Green object for (the class of) the cycle induced by $y$ on $X_{\mathbb{R}}$. The $\mathcal{C}$-arithmetic Chow group $\widehat{\mathrm{CH}}^{p}(X, \mathcal{C})$ is now obtained from the group of $p$-codimensional arithmetic cycles of $(X, \mathcal{C})$ by factoring out by a suitable rational equivalence relation. We prove various properties for these generalized arithmetic Chow groups, emphasizing how the properties of the $\mathcal{G}$-complexes involved are reflected in the properties of the arithmetic Chow groups; a typical example is the ring structure of the direct sum $\bigoplus_{p \geqslant 0} \widehat{\mathrm{CH}}^{p}(X, \mathcal{C})_{\mathbb{Q}}$.

\section{Section 5}

In all the examples of generalized arithmetic Chow groups presented in this paper, the underlying Gillet cohomology will be the Deligne-Beilinson cohomology. Therefore, we recall in the fifth section the basic definitions and facts of Deligne-Beilinson cohomology and homology, which will be needed in the sequel. In particular, we use the fact that real Deligne-Beilinson cohomology can be computed as the sheaf cohomology of the Deligne algebra associated to the Dolbeault algebra of differential forms with logarithmic singularities at infinity. We denote this graded complex of sheaves by $\mathcal{D}_{\log }$. Towards the end of this section, we give explicit representatives for the classes of cycles, in particular for the classes of divisors of sections of (hermitian) line bundles, in real Deligne-Beilinson cohomology in terms of the underlying singular differential forms. Most of the material in this section is well known; we include it for the convenience of the reader.

\section{Section 6}

In the sixth section we use our abstract theory of arithmetic Chow groups to define contravariant and covariant arithmetic Chow groups starting with the Gillet complex $\mathcal{G}=\mathcal{D}_{\text {log }}$. The contravariant Chow groups, which were introduced in $[\mathbf{1 7}]$, are obtained by considering $\mathcal{D}_{\log }$ itself as a $\mathcal{G}$-complex. In this way, we obtain the arithmetic Chow groups $\widehat{\mathrm{CH}}^{*}\left(X, \mathcal{D}_{\log }\right)$. By means of the properties of the Deligne algebra $\mathcal{D}_{\text {log }}$, we find that the direct sum $\bigoplus_{p \geqslant 0} \widehat{\mathrm{CH}}^{p}\left(X, \mathcal{D}_{\log }\right)_{\mathbb{Q}}$ has the structure of a commutative and associative ring. Furthermore, this ring coincides with the arithmetic Chow ring defined by Gillet and Soulé in [36] for arithmetic varieties with projective generic fibre. The covariant Chow groups, which were introduced in [14], are defined using as a $\mathcal{D}_{\text {log-complex a }}$ complex $\mathcal{D}_{\text {cur }}$, which is made out of certain currents and computes real Deligne-Beilinson homology. The properties of the $\mathcal{D}_{\log }$-complex $\mathcal{D}_{\text {cur }}$ show that the arithmetic Chow groups 
$\widehat{\mathrm{CH}}^{*}\left(X, \mathcal{D}_{\text {cur }}\right)$ are covariant for arbitrary proper morphisms and have the structure of a module over the contravariant Chow ring. We end this section with a reformulation of the definition of the height of a cycle, originally given in [10], in the framework of contravariant and covariant arithmetic Chow groups.

\section{Section 7}

In the seventh section we again fix the Deligne algebra $\mathcal{D}_{\log }$ as our Gillet complex $\mathcal{G}$. We then construct a $\mathcal{D}_{\log }$-complex by means of differential forms satisfying (together with their derivatives with respect to $\partial, \bar{\partial}$, and $\partial \bar{\partial}$ ) certain growth conditions of logand log-log-type. We call these differential forms pre-log and pre-log-log forms, respectively, and denote the corresponding $\mathcal{D}_{\text {log-complex by }} \mathcal{D}_{\text {pre }}$. The notation is justified in order to distinguish these differential forms from the forms satisfying the same type of growth conditions together with all their derivatives; the corresponding $\mathcal{D}_{\text {log-complex }}$ will be introduced and studied in $[\mathbf{1 9}]$. By means of the properties of the complex $\mathcal{D}_{\text {pre }}$, the direct sum $\bigoplus_{p \geqslant 0} \widehat{\mathrm{CH}}^{p}\left(X, \mathcal{D}_{\text {pre }}\right)_{\mathbb{Q}}$ has the structure of a commutative and associative ring. This provides the desired higher dimensional extension of the generalized arithmetic intersection pairing introduced in [57]. A useful application of this formalism is the extension of the definition of the height of a cycle with respect to line bundles equipped with a hermitian metric, which becomes logarithmically singular along a divisor with normal crossings. As an illustration, we compute the arithmetic self-intersection number of the line bundle of modular forms on the product of two modular curves equipped with the Petersson metric. Furthermore, we determine the Faltings height of Hecke correspondences on this product of modular curves with respect to the line bundle of modular forms. The same result has been obtained recently by Autissier [3] using a different approach. Related but more elaborate results in the case of Hilbert modular surfaces are contained in the paper [13] mentioned above. The general theory of arithmetic characteristic classes of automorphic vector bundles of arbitrary rank will be developed in $[\mathbf{1 9}]$.

\section{Background results on $K$-theory}

In this section we will review some facts relating to $K$-theory, Chow groups and cohomology theory that will be needed in the sequel. For our purposes, the main interest of $K$-theory is that it provides a method to define the intersection product and the inverse images of algebraic cycles for regular schemes. Most of the results we need are only concerned with the groups $K_{0}$; they are explained in the first chapter of [71]. The main exception to this is Proposition 1.16. Its translation into Chow theory, Theorem 1.34, and cohomology theory will be used in the proof of the fact that the intersection product of arithmetic cycles is well defined. Note however that Proposition 1.16 is proven in [34], and is used in $[\mathbf{3 6}]$.

In this section all schemes will be noetherian, separated and of finite Krull dimension. Given an abelian group $A$ we will write $A_{\mathbb{Q}}=A \otimes \mathbb{Q}$. 


\section{1. $K$-theory}

\section{$K$-theory of schemes}

For any exact category $\mathcal{A}$, Quillen has introduced a simplicial space $\Omega B Q \mathcal{A}$, and has defined the $K$-groups of the category $\mathcal{A}$ as the homotopy groups of this simplicial space (see $[68])$, i.e.

$$
K_{m}(\mathcal{A})=\pi_{m} \Omega B Q \mathcal{A} .
$$

Let $X$ be a scheme (recall that this means a noetherian, separated scheme of finite Krull dimension). Then, we will denote by $\mathcal{M}(X)$ the exact category of coherent sheaves on $X$, and by $\mathcal{P}(X)$ the exact category of locally free coherent sheaves on $X$. We write

$$
\begin{aligned}
& K_{m}^{\prime}(X)=K_{m}(\mathcal{M}(X)), \\
& K_{m}(X)=K_{m}(\mathcal{P}(X)) .
\end{aligned}
$$

In particular, $K_{0}(X)$ is the Grothendieck group of locally free coherent sheaves on $X$, and $K_{0}^{\prime}(X)$ is the Grothendieck group of coherent sheaves on $X$.

We will use many standard facts about the $K$-theory of schemes. For more details the reader is referred, for instance, to $[\mathbf{6 8}]$, or $[\mathbf{7 2}]$. Let us quote some of these facts.

\section{Functoriality of $K$-theory}

The first property we want to quote is the functoriality of $K$-theory. Let $F: \mathcal{A} \rightarrow \mathcal{B}$ be an exact functor between exact categories. Then, there is an induced morphism

$$
\Omega B Q \mathcal{A} \rightarrow \Omega B Q \mathcal{B}
$$

and hence morphisms

$$
K_{m}(\mathcal{A}) \rightarrow K_{m}(\mathcal{B})
$$

From this one can derive the following proposition.

Proposition 1.1. If $f: X \rightarrow X^{\prime}$ is a morphism of schemes, then the inverse image of locally free sheaves induces morphisms

$$
f^{*}: K_{m}\left(X^{\prime}\right) \rightarrow K_{m}(X)
$$

With these morphisms, $K_{*}$ is a contravariant functor between the category of schemes to the category of graded abelian groups.

Proposition 1.2. If $f: Y \rightarrow Y^{\prime}$ is a proper morphism of schemes, then the higher direct images of coherent sheaves induce morphisms

$$
f_{*}: K_{m}^{\prime}(Y) \rightarrow K_{m}^{\prime}\left(Y^{\prime}\right)
$$

With these morphisms, $K_{*}^{\prime}$ is a covariant functor between the category of schemes with proper morphisms and the category of graded abelian groups. 


\section{Localization}

Given exact functors $\mathcal{A} \rightarrow \mathcal{B} \rightarrow \mathcal{C}$ such that the composition $\mathcal{A} \rightarrow \mathcal{C}$ is zero and the induced maps $\Omega B Q(\mathcal{A}) \rightarrow \Omega B Q(\mathcal{B}) \rightarrow \Omega B Q(\mathcal{C})$ form a fibration up to homotopy, then there is a long exact sequence

$$
\cdots \rightarrow K_{m}(\mathcal{A}) \rightarrow K_{m}(\mathcal{B}) \rightarrow K_{m}(\mathcal{C}) \stackrel{\delta}{\rightarrow} K_{m-1}(\mathcal{A}) \rightarrow \cdots .
$$

This applies to the case when $\mathcal{A}$ is a Serre subcategory of $\mathcal{B}$, and $\mathcal{C}=\mathcal{B} / \mathcal{A}$. In particular, this can be used to derive the following result $[\mathbf{6 8}, \S 7,3.2]$.

Proposition 1.3 (localization). Let $X$ be a scheme, $Y \subseteq X$ a closed subscheme, and $U=X \backslash Y$. Then, there is a long exact sequence

$$
\cdots \rightarrow K_{m}^{\prime}(Y) \rightarrow K_{m}^{\prime}(X) \rightarrow K_{m}^{\prime}(U) \stackrel{\delta}{\rightarrow} K_{m-1}^{\prime}(Y) \rightarrow \cdots
$$

$K$ and $K^{\prime}$ of regular schemes

Another important result is the comparison between the groups $K$ and $K^{\prime}[\mathbf{6 8}, \S 7]$.

Proposition 1.4. If $X$ is a regular scheme, then the natural morphism

$$
K_{m}(X) \rightarrow K_{m}^{\prime}(X)
$$

is an isomorphism.

$K$-theory with support

Let $X$ be a scheme, $Y \subseteq X$ a closed subscheme, and $U=X \backslash Y$. Then, the $K$-theory groups of $X$ with support in $Y$ are defined by

$$
\left.K_{m}^{Y}(X)=\pi_{m+1} \text { (homotopy fibre }(B Q \mathcal{P}(X) \rightarrow B Q \mathcal{P}(U))\right) .
$$

By definition, there is a long exact sequence

$$
\cdots \rightarrow K_{m}^{Y}(X) \rightarrow K_{m}(X) \rightarrow K_{m}(U) \stackrel{\delta}{\rightarrow} K_{m-1}^{Y}(X) \rightarrow \cdots .
$$

Combining Proposition 1.4, the above exact sequence and Proposition 1.3 we obtain the following proposition.

Proposition 1.5 (purity). If $X$ is a regular scheme and $Y \subseteq X$ a closed subscheme, then there is a natural isomorphism

$$
K_{m}^{Y}(X) \rightarrow K_{m}^{\prime}(Y)
$$

The purity property clearly implies the following excision property.

Proposition 1.6. Let $X$ be a regular scheme, $Y$ a closed subscheme of $X$, and $V$ an open subscheme of $X$ satisfying $Y \subseteq V$. Then, the restriction map

$$
K_{m}^{Y}(X) \rightarrow K_{m}^{Y}(V)
$$

is an isomorphism. 


\section{2. $K$-theory as generalized sheaf cohomology}

The $K$-theory groups can also be realized as generalized cohomology groups [11]. This allows us to treat $K$-theory and cohomology in a uniform way, and is the basis of the construction by Gillet [34] of characteristic classes for higher $K$-theory. In this section we will briefly recall the definition of generalized sheaf cohomology and how to see higher $K$-theory as generalized sheaf cohomology. We will follow [37] and Appendix B of [46]. There is also a short account in [21]. The reader may also consult the book [50] for a slightly different point of view.

\section{Homotopy theory of spaces}

Let us fix a regular noetherian scheme $S$ of finite Krull dimension. Let $C$ be one of the following sites: $\operatorname{zar}(S)$ the small Zariski site over $S, \operatorname{ZAR}(S)$ the big Zariski site of all schemes of finite type over $S$, or ZAR the big Zariski site of all noetherian schemes of bounded Krull dimension. Let $T=T(C)$ be the topos of sheaves over the site $C$ as in [2]. Let us denote by $s T$ the category of pointed simplicial objects in $T$. The elements of $s T$ will be called spaces. Given a scheme $X$ in the site $C$, we can consider $X$ as a sheaf by

$$
U \mapsto \operatorname{Hom}_{C}(U, X)
$$

We will also denote by $X$ the corresponding constant simplicial object pointed by a disjoint base point.

Definition 1.7. A space is said to be constructed from schemes if all components are representable by a scheme in the site plus a disjoint base point. If $P$ is a property of schemes, we will say that a space $X$ constructed from schemes satisfies $P$, if all the schematic parts of $X$ satisfy $P$.

The following result, due to Joyal, is fundamental for passing to the homotopy category of spaces. A published proof in the non-pointed case can be found in [49] (see [37], and $[46])$.

Proposition 1.8. The category $s T$ is a pointed closed model category in the sense of Quillen [67].

A part of the definition of a closed model category comprises the concepts of weak equivalence, fibrations and cofibrations, and, in particular, the concept of fibrant objects and cofibrant objects. We refer to [46, Appendix B] for the definition of these concepts in the present setting.

Let us denote by $\operatorname{Ho}(s T)$ the homotopy category associated to the closed model category $s T$. If $X$ and $Y$ are spaces, we denote by $[X, Y]$ the morphism in $\operatorname{Ho}(s T)$. If $Y$ is fibrant, it is just the homotopy classes of morphisms. If $Y$ is not fibrant, then $[X, Y]=[X, \widetilde{Y}]$, where $\widetilde{Y}$ is a fibrant space weakly equivalent to $Y$. We will denote by $S X$ and $\Omega X$ the suspension and the loop space of a space $X$, respectively. The loop space functor $\Omega$ is the right adjoint functor of the suspension functor $S$ (see [67]). 


\section{Generalized cohomology}

Definition 1.9. Let $A$ be a space and let $X \in C$. Then, the cohomology space with coefficients in $A$ is defined for $m \geqslant 0$ by

$$
H_{s T}^{-m}(X, A)=\left[S^{m} \wedge X, A\right]
$$

where $S^{m} \wedge X$ is the smash product between the pointed $m$-dimensional sphere $S^{m}$ and $X$. Note that $S^{m} \wedge X$ is canonically isomorphic to the $m$-fold suspension $S^{m} X=S{ }^{m} \cdot S X$.

Remark 1.10. If $X$ is a scheme in the site ZAR and $A$ is an element of $s T(\mathrm{ZAR})$, we will denote also by $A$ the restriction of $A$ to $\operatorname{ZAR}(X)$ or to $\operatorname{zar}(X)$. By the argument at the beginning of the proof of [37, Proposition 5] the cohomology groups $H^{-m}(X, A)$ are the same in the three sites.

\section{Pseudo-flasque presheaves}

If $A$ is a fibrant space, the generalized cohomology can be computed as homotopy groups

$$
H_{s T}^{-m}(X, A)=\left[S^{m} \wedge X, A\right]=\pi_{m}(A(X))=\pi_{m}(\operatorname{Hom}(X, A)) .
$$

More generally, the above equality is a property of pseudo-flasque presheaves.

Definition 1.11. A presheaf $A$ is pseudo-flasque, if $A(U)$ is a fibrant simplicial set for all $U, A(\emptyset)$ is contractible, and for each pair of open sets the diagram

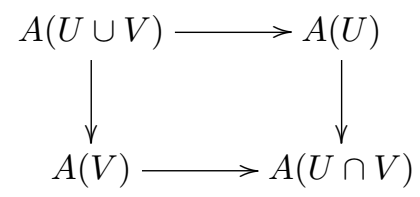

is homotopically Cartesian.

Proposition 1.12 (Brown-Gersten [11]; Gillet-Soulé [37]). Let $A$ be a pseudoflasque presheaf, and let $A^{\prime}$ be the associated sheaf. Then, we have for any scheme $X$ in ZAR

$$
H^{-m}\left(X, A^{\prime}\right)=\pi_{m}(A(X))
$$

\section{K-theory as generalized sheaf cohomology}

Let us denote by $\boldsymbol{K}$ the pointed simplicial sheaf $\mathbb{Z} \times \mathbb{Z}_{\infty}$ BGL (see [37, 3.1]), and by $\boldsymbol{K}^{N}$ the pointed simplicial sheaf $\mathbb{Z} \times \mathbb{Z}_{\infty} \mathrm{BGL}_{N}$. If $X$ is a space, we define for $m \geqslant 0$ its $m$ th $K$-theory group by $H^{-m}(X, \boldsymbol{K})$. We also define the unstable $m$ th $K$-theory group by $H^{-m}\left(X, \boldsymbol{K}^{N}\right)$. In general, $H^{0}\left(X, \boldsymbol{K}^{N}\right)$ are only pointed spaces, but $H^{-m}\left(X, \boldsymbol{K}^{N}\right)$ are abelian groups for $m \geqslant 1$. Following [37], we make the following definition. 
Definition 1.13. We say that a space $X$ is $K$-coherent, if the natural maps

$$
\underset{N}{\lim _{N}} H^{-m}\left(X, \boldsymbol{K}^{N}\right) \rightarrow H^{-m}(X, \boldsymbol{K})
$$

and

$$
\underset{N}{\lim _{\longrightarrow}} H^{m}\left(X, \pi_{n} \boldsymbol{K}^{N}\right) \rightarrow H^{m}\left(X, \pi_{n} \boldsymbol{K}\right)
$$

are isomorphisms for all $m, n \geqslant 0$.

The fact that the groups $H^{-m}(X, \boldsymbol{K})$ agree with the $K$-groups defined by Quillen is proved in [37]. They use the argument of $[\mathbf{1 1}]$ together with some modifications due to the different definitions. The main ingredients are the ' $+=Q$ ' result of Quillen [68], Proposition 1.12, and the fact that the homotopy category used in [11] agrees with the one considered in [37].

Proposition 1.14. Let $X$ be a scheme in the site $C$. Then, there are canonical isomorphisms

$$
K_{m}(X) \rightarrow H^{-m}(X, \boldsymbol{K})
$$

Moreover, $X$ is $K$-coherent.

From the above proposition and a little homological algebra (see [37, 3.2.3], [21, 2.1] and [46, B.2.3]) one can derive the following proposition.

Proposition 1.15. Let $X$ be a noetherian space constructed from schemes of finite Krull dimension (see Definition 1.7) that is degenerate above some simplicial degree, i.e. there exists $N$ such that $X=\operatorname{sk}_{N} X$, where $\operatorname{sk}_{N} X$ is the $N$ th skeleton of $X$. Then, $X$ is $K$-coherent.

The mapping cone

Let $f: X \rightarrow Y$ be a morphism of spaces. We can define the mapping cone $C(X, Y)=$ $C(f)$ of $f$ as the space

$$
C(X, Y)=(Y \amalg X \times I) / \sim,
$$

where $I$ is the simplicial unit interval and $\sim$ is the equivalence relation generated by

$$
\begin{aligned}
& (x, 1) \sim f(x), \\
& (x, 0) \sim *, \\
& (*, t) \sim *,
\end{aligned}
$$

with $*$ denoting the distinguished point in all spaces. Then, we write

$$
H^{-m}(Y, X, \boldsymbol{K})=H^{-m}(C(f), \boldsymbol{K}) .
$$

There is a long exact sequence

$$
\cdots \rightarrow H^{-m}(Y, \boldsymbol{K}) \rightarrow H^{-m}(X, \boldsymbol{K}) \stackrel{\delta}{\rightarrow} H^{-m+1}(Y, X, \boldsymbol{K}) \rightarrow \cdots
$$


The connection morphism of this exact sequence is defined as follows. Let

$$
\alpha: S^{m} \wedge X \rightarrow \boldsymbol{K}
$$

be an element of $H^{-m}(X, \boldsymbol{K})=\left[S^{m} \wedge X, \boldsymbol{K}\right]$. Then, $\delta(\alpha)$ is given as the following composition of maps

$$
\begin{aligned}
S^{m-1} \wedge C(X, Y) \cong C\left(S^{m-1} \wedge\right. & \left.X, S^{m-1} \wedge Y\right) \rightarrow \\
& \quad C\left(S^{m-1} \wedge X, *\right) \cong S^{1} \wedge S^{m-1} \wedge X=S^{m} \wedge X \stackrel{\alpha}{\rightarrow} \boldsymbol{K},
\end{aligned}
$$

where $*$ is the simplicial point; here we also use the fact that the smash product and the mapping cone commute, and that $C(X, *) \cong S^{1} \wedge X$ is the suspension.

$K$-theory with support

Let $X$ be a regular noetherian scheme of finite Krull dimension, $f: U \rightarrow X$ an open immersion, and set $Y=X \backslash U$. The mapping cone of $f$ is a space $C(f) \in s$ ZAR. It is clear that there are canonical isomorphisms

$$
K_{m}^{Y}(X) \rightarrow K_{m}(C(f)), \quad m \geqslant 0 .
$$

\section{Loday product in $K$-theory}

Let us discuss the existence of a product in $K$-theory and its basic properties. The choice of any bijection $\mathbb{N} \times \mathbb{N} \rightarrow \mathbb{N}$ induces a map [59] (see [46, p. 103] for details in this setting)

$$
\left(\mathbb{Z} \times \mathbb{Z}_{\infty} \mathrm{BGL}_{N}\right) \wedge\left(\mathbb{Z} \times \mathbb{Z}_{\infty} \mathrm{BGL}_{N}\right) \rightarrow \mathbb{Z} \times \mathbb{Z}_{\infty} \mathrm{BGL}
$$

Hence, if $X$ and $Y$ are $K$-coherent spaces, there is a well-defined external product

$$
H^{-m}(X, \boldsymbol{K}) \times H^{-n}(Y, \boldsymbol{K}) \rightarrow H^{-m-n}(X \wedge Y, \boldsymbol{K})
$$

via

$$
S^{m+n} \wedge X \wedge Y \cong S^{m} \wedge X \wedge S^{n} \wedge Y \rightarrow \boldsymbol{K}^{N} \wedge \boldsymbol{K}^{N} \rightarrow \boldsymbol{K}
$$

When $X=Y$, composing with the pull-back of the diagonal map $X \rightarrow X \wedge X$, we obtain an associative and graded commutative ring structure (possibly without unit) in $H^{-*}(X, \boldsymbol{K})$. Clearly, the product is functorial.

Let $X, Y$ and $Z$ be $K$-coherent spaces, and let $f: X \rightarrow Y$ be a morphism of spaces. Having defined the product for spaces and using the fact that the mapping cone commutes with the smash product, we observe that there is a product

$$
H^{-m}(Y, X, \boldsymbol{K}) \times H^{-n}(Z, \boldsymbol{K}) \rightarrow H^{-m-n}(Y \wedge Z, X \wedge Z, \boldsymbol{K}) .
$$

The following result is a version of [34, Corollary 7.14]. 
Proposition 1.16. Let $X, Y$ and $Z$ be $K$-coherent spaces, and let $f: X \rightarrow Y$ be a morphism of spaces. If $\alpha \in H^{-m}(X, \boldsymbol{K})$ and $\beta \in H^{-n}(Z, \boldsymbol{K})$, then the Loday product is compatible with long exact sequences, i.e. we have

$$
\begin{aligned}
& \delta(\alpha \cdot \beta)=\delta(\alpha) \cdot \beta \in H^{-m-n+1}(Y \wedge Z, X \wedge Z, \boldsymbol{K}), \\
& \delta(\beta \cdot \alpha)=(-1)^{n} \beta \cdot \delta(\alpha) \in H^{-m-n+1}(Z \wedge Y, Z \wedge X, \boldsymbol{K}) .
\end{aligned}
$$

Proof. The first equality follows from the commutative diagram

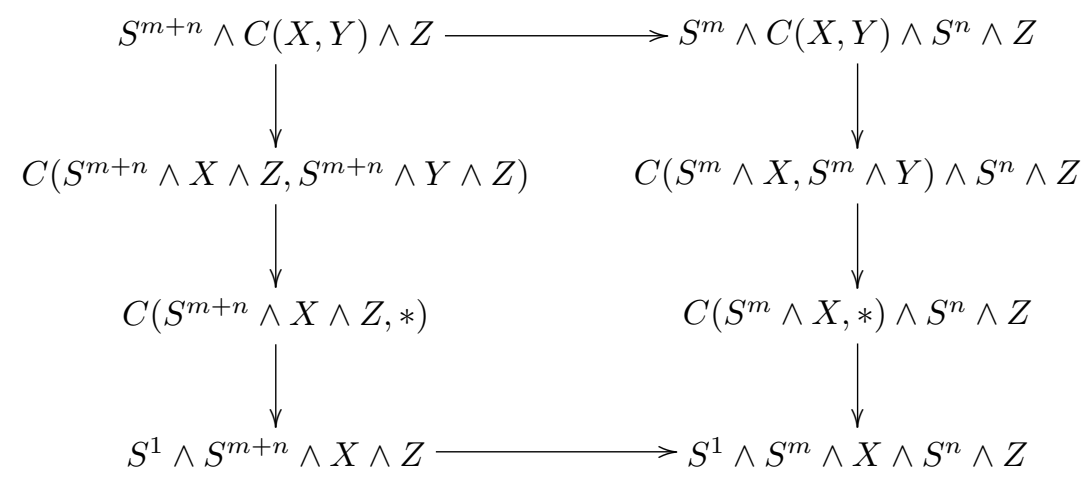

The second statement follows from a similar diagram that involves the isomorphism

$$
S^{n} \wedge S^{1} \wedge S^{m} \rightarrow S^{1} \wedge S^{n} \wedge S^{m}
$$

But identifying both sides with $S^{m+n+1}$, the above isomorphism has degree $(-1)^{n}$.

Products in $K$-theory with support

We will now construct the product of $K$-theory with support. Let $X$ be a scheme in the site, let $U, V$ be open subsets of $X$, and set $Y=X \backslash U, Z=X \backslash V$. Then, there is a pairing

$$
\begin{gathered}
K_{m}^{Y}(X) \otimes K_{n}^{Z}(X)=H^{-m}(C(U, X), \boldsymbol{K}) \otimes H^{-n}(C(V, X), \boldsymbol{K}) \rightarrow \\
H^{-m-n}(C(U, X) \wedge C(V, X), \boldsymbol{K}) .
\end{gathered}
$$

Using the fact that the smash product and the mapping cone commute, we obtain that $C(U, X) \wedge C(V, X)$ is weakly equivalent to either of the spaces

$$
C(C(U \wedge V, U \wedge X), C(X \wedge V, X \wedge X))
$$

or

$$
C(C(U \wedge V, X \wedge V), C(U \wedge X, X \wedge X)) .
$$

We will denote any of them as $C(U \wedge V ; X \wedge V, U \wedge X ; X \wedge X)$. The diagonal map induces a morphism of diagrams

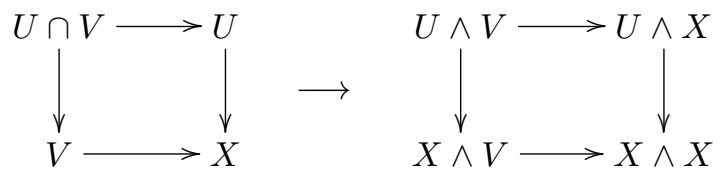


Since $\{U, V\}$ is an open covering of $U \cup V$, we find that $C(U \cap V ; U, V ; X)$ is weakly equivalent to $C(U \cup V, X)$. Therefore, we obtain a pairing

$$
K_{m}^{Y}(X) \otimes K_{n}^{Z}(X) \stackrel{\cup}{\rightarrow} H^{-m-n}(C(U \cup V, X), \boldsymbol{K})=K_{m+n}^{Y \cap Z}(X) .
$$

A consequence of Proposition 1.16 is the following proposition.

Proposition 1.17. Let $X$ be a scheme in the site, let $U, V$ be open subsets of $X$, and set $Y=X \backslash U, Z=X \backslash V$. Then, the following diagram commutes:

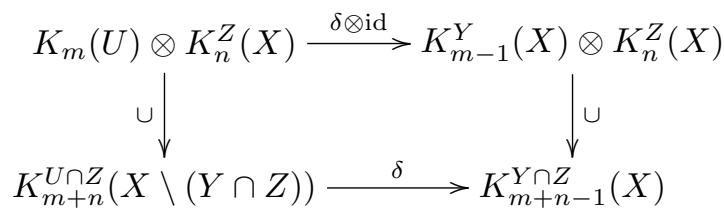

here we have identified $K_{m+n}^{U \cap Z}(U)$ with $K_{m+n}^{U \cap Z}(X \backslash(Y \cap Z))$ using excision, and $\delta$ is the connection morphism.

Direct images and the projection formula

Let $f: X \rightarrow X^{\prime}$ be a proper morphism of regular schemes in the site. Let $Z \subseteq X$ be a closed subscheme. The isomorphisms $K_{m}^{\prime}(Z) \rightarrow K_{m}^{Z}(X)$ and $K_{m}^{\prime}(f(Z)) \rightarrow K_{m}^{f \overline{(Z)}}\left(X^{\prime}\right)$ together with the direct image of the $K^{\prime}$-groups induce direct image morphisms

$$
f_{*}: K_{m}^{Z}(X) \rightarrow K_{m}^{f(Z)}\left(X^{\prime}\right) .
$$

These morphisms satisfy the projection formula.

Proposition 1.18. Let $f: X \rightarrow X^{\prime}$ be a proper morphism of regular schemes. Let $Z \subseteq X$, and $Z^{\prime} \subseteq X^{\prime}$ be closed subschemes. Then, for $\alpha \in K_{m}^{Z^{\prime}}\left(X^{\prime}\right)$ and $\beta \in K_{n}^{Z}(X)$, we have

$$
f_{*}\left(f^{*}(\alpha) \cup \beta\right)=\alpha \cup f_{*}(\beta) \in K_{m+n}^{f(Z) \cap Z^{\prime}}\left(X^{\prime}\right) .
$$

\section{Gersten-Quillen spectral sequence}

Let $X$ be a regular scheme in the site. Let us denote by $X^{(p)}$ the set of $p$-codimensional points of $X$. If $x \in X^{(p)}$, we will denote

$$
K_{m}^{x}(X)=\lim _{\rightarrow} K_{m}^{\overline{\{x\}} \cap U}(U),
$$

where the limit is taken over all open subsets $U$ of $X$ containing the point $x$.

Theorem 1.19. Let $X$ be a regular scheme in ZAR. Then, there exists a spectral sequence

$$
E_{r}^{p, q}(X) \Longrightarrow K_{-p-q}(X)
$$


with

$$
E_{1}^{p, q}(X)=\bigoplus_{x \in X^{(p)}} K_{-p-q}^{x}(X)
$$

Moreover, this spectral sequence is convergent (since $X$ has finite Krull dimension).

Observe, moreover, that, using purity, we can identify $K_{-p-q}^{x}(X)$ with $K_{-p-q}(k(x))$, where $k(x)$ is the residue field at the point $x$. See $[\mathbf{3 4}]$ for a proof of this theorem for generalized cohomology.

\section{3. $\lambda$-structure in $K$-theory and absolute cohomology}

$\lambda$-rings and $\lambda$-algebras

Recall that a $\lambda$-ring with involution is a ring $R$ equipped with a family of operators $\left\{\lambda^{k}\right\}_{k \geqslant 0}$ and an involution $\psi^{-1}$ satisfying certain relations (see [5]). In particular, any $\lambda$-ring has a unit. Let $R$ be a $\lambda$-ring with involution, and $A$ an $R$-algebra. We call $A$ a $\lambda$-R-algebra with involution, if it is equipped with a family of operators $\left\{\lambda^{k}\right\}_{k \geqslant 1}$ and an involution $\psi^{-1}$ such that $R \oplus A$ is a $\lambda$-ring (see [54] and [46]).

$\lambda$-structure in $K$-theory

For any $N \geqslant 1$, one can define a family of operators $\left\{\lambda_{N}^{k}\right\}_{k \geqslant 1}($ see $[\mathbf{3 7}, \S 4])$

$$
\lambda_{N}^{k}: \mathbb{Z} \times \mathbb{Z}_{\infty} \mathrm{BGL}_{N} \rightarrow \mathbb{Z} \times \mathbb{Z}_{\infty} \text { BGL }
$$

which are compatible with the inclusions $\mathrm{BGL}_{N-1} \rightarrow \mathrm{BGL}_{N}$. Therefore, for any space $X$, there are induced operators

$$
\lambda_{N}^{k}: H^{-m}\left(X, \boldsymbol{K}^{N}\right) \rightarrow H^{-m}(X, \boldsymbol{K})
$$

If the space $X$ is $K$-coherent, we have induced operators

$$
\lambda^{k}: H^{-m}(X, \boldsymbol{K}) \rightarrow H^{-m}(X, \boldsymbol{K})
$$

In the same way one can define an involution $\psi^{-1}$.

Let $S^{0}$ be the simplicial pointed 0 -sphere. Following [46], we will write $K_{0}(s T)=$ $H^{0}\left(S^{0}, \boldsymbol{K}\right)$. This is a $\lambda$-ring. If the site $C$ equals $\operatorname{ZAR}(S)$, we have $K_{0}(s T)=K_{0}(S)$. A proof of the following theorem can be found in $[\mathbf{3 7}]$ (see also $[\mathbf{4 6}]$ ).

Theorem 1.20. Let $X$ be a $K$-coherent space. The family of operators $\left\{\lambda^{k}\right\}_{k \geqslant 1}$ together with the involution $\psi^{-1}$ turn $H^{-m}(X, \boldsymbol{K})$ into a $\lambda-K_{0}(s T)$-algebra with involution. Moreover, $H^{0}(X, \boldsymbol{K})$ is provided with a natural augmentation

$$
\epsilon: H^{0}(X, \boldsymbol{K}) \rightarrow H^{0}(X, \mathbb{Z})
$$


Adams operations and $\gamma$-filtration

Let $\Phi$ be the monoid of natural operations on $\lambda$-rings with an involution. Among the operations in $\Phi$, there are the $\gamma$-operations $\gamma^{k}, k \geqslant 1$, and the Adams operations $\psi^{k}$, $k \in \mathbb{Z} \backslash\{0\}$. These operations are defined by the usual formulae

$$
\begin{gathered}
\gamma^{k}(x)=\lambda^{k}(x+k-1), \quad k \geqslant 1, \\
\psi^{k}-\lambda^{1} \psi^{k-1}+\cdots+(-1)^{k-1} \lambda^{k-1} \psi^{1}+(-1)^{k} \lambda^{k}=0, \quad k \geqslant 1,
\end{gathered}
$$

and $\psi^{-k}=\psi^{-1} \circ \psi^{k}$. Since these operations do not involve the unit, they are also operations of $\lambda$-algebras. By means of the $\gamma$-operations we can define a filtration in the $K$-groups.

Definition 1.21. Let $X$ be a $K$-coherent space. Then $F_{\gamma}^{i} H^{-m}(X, \boldsymbol{K})$ is defined as the subgroup of $H^{-m}(X, \boldsymbol{K})$ generated by the products

$$
\gamma^{i(1)}\left(x_{1}\right) \cdots \gamma^{i(r)}\left(x_{r}\right)
$$

where $i(1)+\cdots+i(r) \geqslant i, x_{j} \in H^{-m(j)}(X, \boldsymbol{K})$ with $m(1)+\cdots+m(r)=m$ and $\epsilon\left(x_{j}\right)=0$.

The following properties of the Adams operations are well known.

Proposition 1.22. The Adams operations respect the additive structure of $K$-theory, the Loday product and pull-backs. Moreover, we have $\psi^{k} \circ \psi^{k^{\prime}}=\psi^{k+k^{\prime}}$.

Observe that the $\lambda$-structure in $K$-theory involves only the product between $K_{0}$ and higher $K$-theory and not the Loday product. Nevertheless, in order to see that the Adams operations are compatible with the Loday product one can argue as in [54] (see also [21]).

Eigenspaces for the Adams operations

Let us write

$$
H^{-m}(X, \boldsymbol{K})^{(i)}=\left\{x \in H^{-m}(X, \boldsymbol{K}) \otimes \mathbb{Q} \mid \psi^{k}(x)=k^{i} x, \forall k \in \mathbb{Z} \backslash\{0\}\right\} .
$$

We will also write $K_{m}(X)^{(i)}=H^{-m}(X, \boldsymbol{K})^{(i)}$. The following proposition is proven in $[37]$.

Proposition 1.23. Let $X$ be a $K$-coherent space of dimension at most $d$. Then, for $m \geqslant 0$, there is a decomposition

$$
H^{-m}(X, \boldsymbol{K}) \otimes \mathbb{Q}=\sum_{i=\alpha}^{m+d} H^{-m}(X, \boldsymbol{K})^{(i)},
$$

where $\alpha=\min (m, 2)$. Moreover, we have

$$
H^{-m}(X, \boldsymbol{K})^{(i)}=\operatorname{Gr}_{\gamma}^{i} H^{-m}(X, \boldsymbol{K}) \otimes \mathbb{Q} .
$$


Riemann-Roch without denominators

The Riemann-Roch theorem without denominators studies the effect of closed immersions on the operations in $K$-theory. Let $X$ and $Y$ be regular schemes in $\operatorname{ZAR}(S)$, let $j: Y \rightarrow X$ be a closed immersion, and let us denote by $N=N_{X / Y}$ the class of the normal bundle of $Y$ in $X$ in $K_{0}(Y)$. For any operation $\tau \in \Phi$, let us write $\tau(N, x)$ as in $[\mathbf{7 0}, \S 4]$. Let $Z$ be a closed subset of $Y$. Using the identifications $K_{m}^{\prime}(Z)=K_{m}^{Z}(Y)$ and $K_{m}^{\prime}(Z)=K_{m}^{Z}(X)$, we obtain an isomorphism

$$
j_{*}: K_{m}^{Z}(Y) \rightarrow K_{m}^{Z}(X)
$$

Theorem 1.24 (Soulé [70]). For $x \in K_{m}^{Z}(Y)$ and $\tau \in \Phi$, we have

$$
\tau\left(j_{*}(x)\right)=j_{*}(\tau(N, x)) .
$$

Remark 1.25. This result is generalized in $[\mathbf{2 1}]$ and [46] to a certain class of closed immersions of spaces. Nevertheless, in these papers the schemes are assumed to be smooth over the base $S$.

Direct images and the gamma filtration

Proposition 1.26. Let $f: X \rightarrow X^{\prime}$ be a proper morphism between regular quasiprojective schemes of $\operatorname{ZAR}(S)$ of dimension $d$ and $d^{\prime}$, respectively. Let $Z$ be a closed subscheme of $X$. Then, there is an inclusion

$$
f_{*}\left(F_{\gamma}^{j} K_{m}^{Z}(X) \otimes \mathbb{Q}\right) \subseteq F_{\gamma}^{j+d^{\prime}-d} K_{m}^{f(Z)}\left(X^{\prime}\right) \otimes \mathbb{Q}
$$

Proof. The Riemann-Roch theorem [70, 7.2] states that there are increasing filtrations $F$. of $K_{m}^{\prime}(Z) \otimes \mathbb{Q}$ and $K_{m}^{\prime}(f(Z)) \otimes \mathbb{Q}$ satisfying $f_{*}\left(F_{j}\right) \subseteq F_{j}$. These filtrations are defined as follows. One chooses a closed immersion $i: Z \rightarrow M$, where $M$ is an equidimensional scheme of dimension $n$, smooth and surjective over the base $S$. Then, there is an isomorphism $i_{*}: K_{m}(Z) \rightarrow K_{m}^{Z}(M)$, and we have

$$
F_{j} K_{m}^{\prime}(Z) \otimes \mathbb{Q}=i_{*}^{-1}\left(F_{\gamma}^{n-j} K_{m}^{Z}(M)\right)
$$

We can choose $M$ such that the inclusion $i$ factors as $Z \stackrel{i^{\prime}}{\hookrightarrow} X \stackrel{j}{\hookrightarrow} M$. Using the RiemannRoch theorem without denominators (as in the proof of the independence of $M$ in [70, $7.2,3])$ one obtains that $j_{*}\left(F_{\gamma}^{j} K_{m}^{Z}(X) \otimes \mathbb{Q}\right)=F_{\gamma}^{j-d+n} K_{m}^{Z}(M)$. This leads to

$$
F_{\gamma}^{j} K_{m}^{Z}(X) \otimes \mathbb{Q}=F_{d-j} K_{m}^{\prime}(Z) \otimes \mathbb{Q}
$$

which implies the claim. 


\section{Absolute cohomology}

The graded pieces for the $\gamma$-filtration tensored by $\mathbb{Q}$ or, put another way, the eigenspaces for the Adams operations, form a cohomology theory. By the RiemannRoch theorem for higher $K$-theory given in [34], it is a universal cohomology. One can define it for coherent spaces satisfying certain technical conditions. Since we will not need it in the sequel, we will define it only for a pair of schemes.

Definition 1.27. Let $X$ be a regular scheme in the site and let $Y$ be a closed subset of $X$. Then, the absolute cohomology groups of $X$ with support on $Y$ are defined by

$$
H_{\mathcal{A}, Y}^{2 i-m}(X, \mathbb{Q}(i))=K_{m}^{Y}(X)^{(i)}
$$

Let us summarize the basic properties of absolute cohomology. The next theorem follows easily from the properties of $K$-theory and the Adams operations.

\section{Theorem 1.28.}

(i) Let $X$ be a regular scheme in ZAR, and let $Y, Z$ be closed subsets of $X$. Then, the product in $K$-theory induces a product

$$
H_{\mathcal{A}, Y}^{m}(X, \mathbb{Q}(i)) \otimes H_{\mathcal{A}, Z}^{n}(X, \mathbb{Q}(j)) \stackrel{\cup}{\rightarrow} H_{\mathcal{A}, Y \cap Z}^{m+n}(X, \mathbb{Q}(i+j)) .
$$

(ii) Let $f: X^{\prime} \rightarrow X$ be a morphism of regular schemes in ZAR. Let $Y$ be a closed subset of $X$, and $Y^{\prime}$ a closed subset of $X^{\prime}$ satisfying $f^{-1}(Y) \subseteq Y^{\prime}$. Then, there is pull-back morphism

$$
f^{*}: H_{\mathcal{A}, Y}^{n}(X, \mathbb{Q}(i)) \rightarrow H_{\mathcal{A}, Y^{\prime}}^{n}\left(X^{\prime}, \mathbb{Q}(i)\right)
$$

respecting the multiplicative structure and turning absolute cohomology into a contravariant functor.

(iii) Let $X$ be a regular scheme in ZAR, and $Y$ closed a subset of $X$. Then, there is a long exact sequence

$$
\cdots \rightarrow H_{\mathcal{A}}^{n}(X, \mathbb{Q}(i)) \rightarrow H_{\mathcal{A}}^{n}(X \backslash Y, \mathbb{Q}(i)) \stackrel{\delta}{\rightarrow} H_{\mathcal{A}, Y}^{n+1}(X, \mathbb{Q}(i)) \rightarrow \cdots
$$

(iv) Let $X$ be a regular scheme in ZAR, let $Y, Z$ be closed subsets of $X$, and set $U=X \backslash Y$. Then, the following diagram commutes:

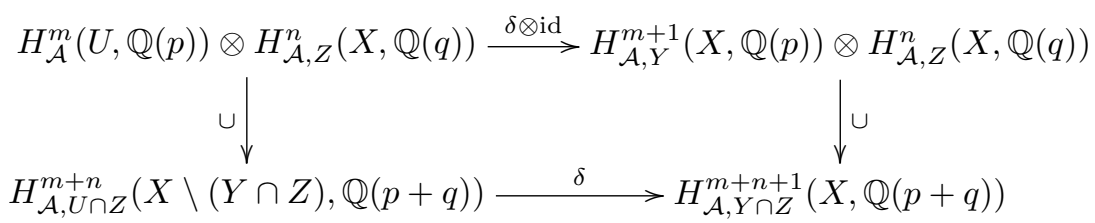


(v) Let $f: X \rightarrow X^{\prime}$ be a proper morphism of regular, equidimensional, quasi-projective schemes in $\operatorname{ZAR}(S)$ of dimension $d$ and $d^{\prime}$, respectively. Let $Z$ be a closed subscheme of $X$. Then, there are morphisms

$$
f_{*}: H_{\mathcal{A}, Z}^{n}(X, \mathbb{Q}(p)) \rightarrow H_{\mathcal{A}, f(Z)}^{n-2 d+2 d^{\prime}}\left(X^{\prime}, \mathbb{Q}\left(p-d+d^{\prime}\right)\right) .
$$

If $g: X^{\prime} \rightarrow X^{\prime \prime}$ is another proper morphism, then $(g \circ f)_{*}=g_{*} \circ f_{*}$. Moreover, if $Z^{\prime}$ is a closed subscheme of $X^{\prime}, \alpha \in H_{\mathcal{A}, Z^{\prime}}^{m}\left(X^{\prime}, \mathbb{Q}(p)\right)$, and $\beta \in H_{\mathcal{A}, Z}^{n}(X, \mathbb{Q}(q))$, we have

$$
f_{*}\left(f^{*}(\alpha) \cup \beta\right)=\alpha \cup f_{*}(\beta) \in H_{\mathcal{A}, f(Z) \cap Z^{\prime}}^{m+n-2 d+2 d^{\prime}}\left(X^{\prime}, \mathbb{Q}\left(p+q-d+d^{\prime}\right)\right) .
$$

The Adams operations and the Gersten-Quillen spectral sequence

Since the Adams operations are defined on the level of sheaves of simplicial spaces, they induce morphisms of Gersten-Quillen spectral sequences. Therefore, after tensoring with $\mathbb{Q}$, the Quillen spectral sequence splits as a direct sum of spectral sequences

$$
E_{r}^{p, q}(X)^{(i)} \Longrightarrow K_{-p-q}(X)^{(i)},
$$

where $E_{r}^{p, q}(X)^{(i)} \subseteq E_{r}^{p, q}(X)_{\mathbb{Q}}$ is the eigenspace of $\psi^{k}$ of eigenvalue $k^{i}$. By the RiemannRoch theorem without denominators (see Theorem 1.24, or [70, Theorem 4]), we have for $x \in X^{(p)}$

$$
K_{m}^{x}(X)^{(i)}=K_{m}(k(x))^{(i-p)} .
$$

Therefore, we have the equality

$$
E_{1}^{p, q}(X)^{(i)}=\bigoplus_{x \in X^{(p)}} K_{-p-q}(k(x))^{(i-p)} .
$$

Since we have for a field $k$ (see [70])

$$
\begin{aligned}
& K_{0}(k)_{\mathbb{Q}}=K_{0}(k)^{(0)}, \\
& K_{1}(k)_{\mathbb{Q}}=K_{1}(k)^{(1)} \text { and } \\
& K_{2}(k)_{\mathbb{Q}}=K_{2}(k)^{(2)},
\end{aligned}
$$

we obtain the following proposition.

Proposition 1.30. Let $X$ be a regular scheme in the site. Then, the lines $p=-q$ and $p=-q-1$ of the spectral sequence $E_{r}^{p, q}(X)_{\mathbb{Q}}$ degenerate at the term $E_{2}$. Thus there are natural isomorphisms

$$
\begin{aligned}
& E_{2}^{p,-p}(X)_{\mathbb{Q}} \cong K_{0}(X)^{(p)}, \\
& E_{2}^{p-1,-p}(X)_{\mathbb{Q}} \cong K_{1}(X)^{(p)} .
\end{aligned}
$$


Proof. By (1.28) we have $E_{1}^{p,-p}(X)_{\mathbb{Q}}=E_{1}^{p,-p}(X)^{(p)}$. Again by (1.28) we have for $r \geqslant 2$

$$
E_{1}^{p-r,-p+r-1}(X)^{(p)}=\bigoplus_{x \in X^{(p-r)}} K_{1}(k(x))^{(r)}=0 .
$$

This leads to the isomorphism $E_{2}^{p,-p}(X)_{\mathbb{Q}} \cong K_{0}(X)^{(p)}$. The same argument applies to the line $p=-q-1$.

Note that in $[\mathbf{7 0}]$ it is also proven that the line $p=-q-2$ degenerates at the term $E_{2}$. A consequence of Proposition 1.30 is the following.

Proposition 1.31. Let $X$ be a regular scheme in the site. Then, there are natural isomorphisms

$$
\begin{aligned}
E_{2}^{p,-p}(X)_{\mathbb{Q}} & \cong H_{\mathcal{A}}^{2 p}(X, \mathbb{Q}(p)), \\
E_{2}^{p-1,-p}(X)_{\mathbb{Q}} & \cong H_{\mathcal{A}}^{2 p-1}(X, \mathbb{Q}(p)) .
\end{aligned}
$$

\subsection{Chow Groups of regular schemes}

$K$-chains and Chow groups

Let $X$ be a regular scheme in ZAR. Let us denote

$$
R_{p}^{q}(X)=E_{1}^{q,-p}(X)=\bigoplus_{x \in X^{(q)}} K_{p-q}(k(x))
$$

where $E_{1}^{q,-p}(X)$ are the terms of the Gersten-Quillen spectral sequence.

Recall that $K_{0}(k(x))=\mathbb{Z}$, and $K_{1}(k(x))=k^{*}(x)$. Therefore, the group $R_{p}^{p}(X)$ equals the group $\mathrm{Z}^{p}(X)$ of $p$-codimensional cycles on $X$. Any element $f \in R_{p}^{p-1}(X)$ can be written as $f=\sum_{x \in X^{(p-1)}} f_{x}$ with $f_{x} \in k^{*}(x)$. The elements of $R_{p}^{p-1}(X)$ are called $K_{1^{-}}$ chains. As usual we denote by $\mathrm{CH}^{p}(X)$ the $p$ th Chow group of $X$. In $[6 \mathbf{6 8}, \S 5]$, the following proposition is proven.

Proposition 1.32. The differential d : $R_{p}^{p-1}(X) \rightarrow R_{p}^{p}(X)$ in the Gersten-Quillen spectral sequence is given by $\mathrm{d} f=\operatorname{div}(f)=\sum_{x \in X^{(p-1)}} \operatorname{div}\left(f_{x}\right)$.

Therefore, we obtain an identification

$$
\mathrm{CH}^{p}(X)=R_{p}^{p}(X) / \mathrm{d} R_{p}^{p-1}(X)=E_{2}^{p,-p}(X) .
$$

Hence, by Proposition 1.31, we obtain a natural isomorphism

$$
\mathrm{CH}^{p}(X)_{\mathbb{Q}} \cong H_{\mathcal{A}}^{2 p}(X, \mathbb{Q}(p)) .
$$

The discrepancy in sign between Proposition 1.32 and [17, p. 365] stems from the fact that we are using a different convention for the connecting morphism (see Remark 2.71). 
Let us write

$$
\mathrm{CH}^{p-1, p}(X)=\mathrm{Z} R_{p}^{p-1}(X) / \mathrm{d} R_{p}^{p-2}(X)=E_{2}^{p-1,-p}(X),
$$

where $\mathrm{Z} R_{p}^{p-1}(X)=\operatorname{Ker}\left(\mathrm{d}: R_{p}^{p-1}(X) \rightarrow R_{p}^{p}(X)\right)$ is the subgroup of cycles. Then, again by Proposition 1.31, we have an isomorphism

$$
\mathrm{CH}^{p-1, p}(X)_{\mathbb{Q}} \cong H_{\mathcal{A}}^{2 p-1}(X, \mathbb{Q}(p)) .
$$

Chow groups with support

For a closed subset $Y \subseteq X$, we define

$$
R_{p, Y}^{q}(X)=\bigoplus_{x \in X^{(q)} \cap Y} K_{p-q}(k(x)) .
$$

In particular, $R_{p, Y}^{p}(X)$ equals the group $\mathrm{Z}_{Y}^{p}(X)$ of $p$-codimensional cycles on $X$ with support on $Y$. We write

$$
\begin{aligned}
\mathrm{CH}_{Y}^{p}(X) & =R_{p, Y}^{p}(X) / \mathrm{d} R_{p, Y}^{p-1}(X), \\
\mathrm{CH}_{Y}^{p-1, p}(X) & =\mathrm{Z} R_{p, Y}^{p-1}(X) / \mathrm{d} R_{p, Y}^{p-2}(X) .
\end{aligned}
$$

We call $\mathrm{CH}_{Y}^{p}(X)$ the Chow group of $p$-codimensional cycles on $X$ with support on $Y$. As before we have isomorphisms

$$
\begin{aligned}
& \mathrm{CH}_{Y}^{p}(X)_{\mathbb{Q}} \cong E_{2, Y}^{p,-p}(X)_{\mathbb{Q}} \cong H_{\mathcal{A}, Y}^{2 p}(X, \mathbb{Q}(p)), \\
& \mathrm{CH}_{Y}^{p-1, p}(X)_{\mathbb{Q}} \cong E_{2, Y}^{p-1,-p}(X)_{\mathbb{Q}} \cong H_{\mathcal{A}, Y}^{2 p-1}(X, \mathbb{Q}(p)) .
\end{aligned}
$$

Writing $U=X \backslash Y$, we have a long exact sequence

$$
\mathrm{CH}^{p-1, p}(U) \stackrel{\delta}{\rightarrow} \mathrm{CH}_{Y}^{p}(X) \rightarrow \mathrm{CH}^{p}(X) \rightarrow \mathrm{CH}^{p}(U) \rightarrow 0 .
$$

A family $\varphi$ of supports on $X$ is a family of closed subsets of $X$ such that, if $Y, Z \in \varphi$, then $Y \cup Z \in \varphi$. For any family $\varphi$ of supports on $X$, we define

$$
\begin{aligned}
R_{p, \varphi}^{q}(X) & =\lim _{Y \in \varphi} R_{p, Y}^{q}(X), \\
\mathrm{CH}_{\varphi}^{p}(X) & =\underset{Y \in \varphi}{\lim _{Y \in \varphi}} \mathrm{CH}_{Y}^{p}(X), \\
\mathrm{CH}_{\varphi}^{p-1, p}(X) & =\underset{Y \in \varphi}{\lim _{Y \in \varphi}} \mathrm{CH}_{Y}^{p-1, p}(X), \\
H_{\mathcal{A}, \varphi}^{n}(X, \mathbb{Q}(p)) & =\underset{Y \in \varphi}{\lim _{Y \in \varphi}} H_{\mathcal{A}, Y}^{n}(X, \mathbb{Q}(p)) .
\end{aligned}
$$

We have the isomorphisms

$$
\begin{aligned}
& \mathrm{CH}_{\varphi}^{p}(X)_{\mathbb{Q}} \cong H_{\mathcal{A}, \varphi}^{2 p}(X, \mathbb{Q}(p)), \\
& \mathrm{CH}_{\varphi}^{p-1, p}(X)_{\mathbb{Q}} \cong H_{\mathcal{A}, \varphi}^{2 p-1}(X, \mathbb{Q}(p)) .
\end{aligned}
$$


Intersection of cycles on regular schemes

A method of defining products for the Chow groups of regular schemes, where no moving lemma is available and it is not possible to use the deformation to the normal cone technique, is given by means of the isomorphism between Chow groups and absolute cohomology groups. In this way we can transfer the multiplicative properties of $K$-theory to the Chow groups. Namely, we define a pairing

$$
\mathrm{CH}_{Y}^{p}(X)_{\mathbb{Q}} \otimes \mathrm{CH}_{Z}^{q}(X)_{\mathbb{Q}} \dot{\rightarrow} \mathrm{CH}_{Y \cap Z}^{p+q}(X)_{\mathbb{Q}}
$$

by means of the commutative diagram

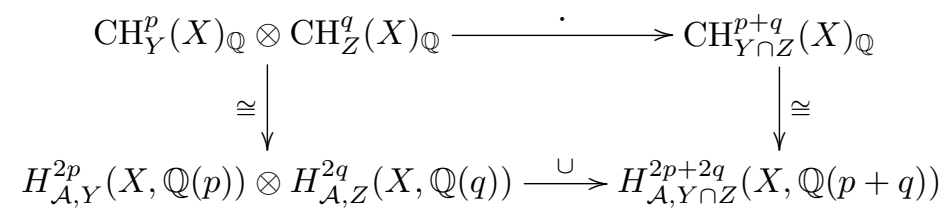

Theorem 1.33 (Gillet-Soulé [35]). If $X$ is a regular scheme of finite Krull dimension, and $Y, Z$ are closed subsets, then the above pairing

$$
\mathrm{CH}_{Y}^{p}(X)_{\mathbb{Q}} \otimes \mathrm{CH}_{Z}^{q}(X)_{\mathbb{Q}} \dot{\rightarrow} \mathrm{CH}_{Y \cap Z}^{p+q}(X)_{\mathbb{Q}}
$$

satisfies the following properties.

(i) It turns $\bigoplus_{Y, p} \mathrm{CH}_{Y}^{p}(X)_{\mathbb{Q}}$ into a commutative ring with unit $[X] \in \mathrm{CH}^{0}(X)$.

(ii) It is compatible with the change of support maps

$$
\mathrm{CH}_{Y}^{p}(X)_{\mathbb{Q}} \rightarrow \mathrm{CH}_{Y^{\prime}}^{p}(X)_{\mathbb{Q}}
$$

whenever $Y \subseteq Y^{\prime} \subseteq X$.

(iii) Let $y \in \mathrm{CH}_{Y}^{p}(X)_{\mathbb{Q}}, z \in \mathrm{CH}_{Z}^{q}(X)_{\mathbb{Q}}$ with $Y=\operatorname{supp} y, Z=\operatorname{supp} z$, and $Y, Z$ having proper intersection. Then, their intersection product $y \cdot z \in \mathrm{CH}_{Y \cap Z}^{p+q}(X)_{\mathbb{Q}}$ is given by Serre's Tor-formula

$$
y \cdot z=\left[\sum_{x \in Y \cap Z}\left(\sum_{i \geqslant 0}(-1)^{i} \ell_{\mathcal{O}_{X, x}}\left(\operatorname{Tor}_{i}^{\mathcal{O}_{X, x}}\left(\mathcal{O}_{Y, x}, \mathcal{O}_{Z, x}\right)\right)\right) \overline{\{x\}}\right],
$$

where $\ell_{\mathcal{O}_{X, x}}$ denotes the length of a $\mathcal{O}_{X, x}$-module.

It is clear from this result that, if $\varphi$ and $\psi$ are two families of supports, then there is an induced pairing

$$
\mathrm{CH}_{\varphi}^{p}(X)_{\mathbb{Q}} \otimes \mathrm{CH}_{\psi}^{q}(X)_{\mathbb{Q}} \stackrel{\cdot}{\rightarrow} \mathrm{CH}_{\varphi \cap \psi}^{p+q}(X)_{\mathbb{Q}},
$$

where $\varphi \cap \psi$ is the family of supports given by

$$
\varphi \cap \psi=\{Y \cap Z \mid Y \in \varphi, Z \in \psi\} .
$$

We will also need products between $\mathrm{CH}^{p-1, p}(X)$ and $\mathrm{CH}^{q}(X)$. 
Theorem 1.34. Let $X$ be a regular scheme, let $Y, Z$ be closed subsets of $X$, and set $U=X \backslash Y$. Then, there exists a well-defined pairing

$$
\mathrm{CH}^{p-1, p}(U)_{\mathbb{Q}} \otimes \mathrm{CH}_{Z}^{q}(X)_{\mathbb{Q}} \stackrel{\cdot}{\rightarrow} \mathrm{CH}_{U \cap Z}^{p+q-1, p+q}(X \backslash(Y \cap Z))_{\mathbb{Q}}
$$

such that the diagram

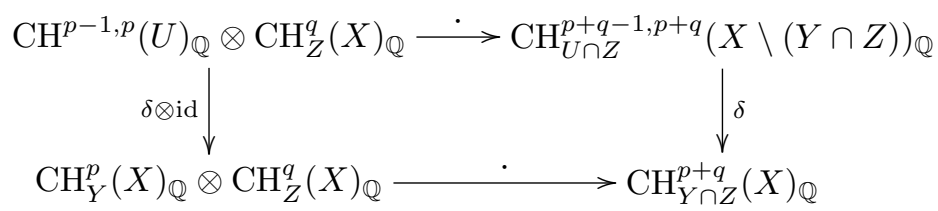

commutes.

Proof. The pairing is defined by imposing the commutativity of the diagram

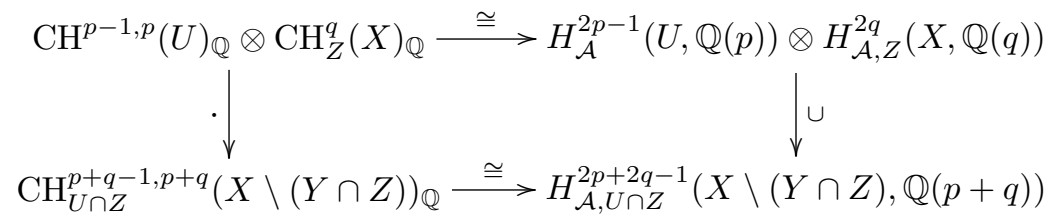

The compatibility of the pairing with the connection morphism now follows from Theorem 1.28 (iv).

For a $K_{1}$-chain $f \in R_{p}^{p-1}(X)$, put $y=\operatorname{div}(f), Y=\operatorname{supp} y$, and $U=X \backslash Y$; observe that $f$ determines an element $[f] \in \mathrm{CH}^{p-1, p}(U)$. Let $z$ be a $q$-codimensional cycle with $Z=\operatorname{supp} z$. By the above theorem, there is a well-defined element

$$
[f] \cdot[z] \in \mathrm{CH}_{U \cap Z}^{p+q-1, p+q}(X \backslash(Y \cap Z))_{\mathbb{Q}} .
$$

Using that $R_{p+q}^{p+q-1}(X \backslash(Y \cap Z))$ is a direct summand of $R_{p+q}^{p+q-1}(X)$, the above theorem implies the following corollary.

Corollary 1.35. Assume that $U \cap Z \neq \emptyset$. Then, there exists a $K_{1}$-chain $g \in R_{p+q}^{p+q-1}(X)_{\mathbb{Q}}$, determined up to $\mathrm{d} R_{p+q}^{p+q-2}(X)_{\mathbb{Q}}+R_{p+q, Y \cap Z}^{p+q-1}(X)_{\mathbb{Q}}$, such that its class in $\mathrm{CH}_{U \cap Z}^{p+q-1, p+q}(X \backslash$ $(Y \cap Z))_{\mathbb{Q}}$ is equal to $[f] \cdot[z]$. Moreover, we have

$$
[\operatorname{div}(g)]=[\operatorname{div}(f)] \cdot[z]
$$

in the group $\mathrm{CH}_{Y \cap Z}^{p+q}(X)_{\mathbb{Q}}$.

Remark 1.37. If we have $\operatorname{codim}(Y \cap Z)=p+q$ in Corollary 1.35, the equality $\mathrm{CH}_{Y \cap Z}^{p+q}(X)=\mathrm{Z}_{Y \cap Z}^{p+q}(X)$ shows that identity (1.35) holds true on the level of cycles, i.e. we have

$$
\operatorname{div}(g)=\operatorname{div}(f) \cdot z .
$$




\section{Pull-back}

Here we recall the properties of the pull-back of algebraic cycles and $K_{1}$-chains by a quasi-projective morphism of regular schemes. Let us start with the case of cycles. Since any such morphism can be factored as the composition of a closed immersion and a flat morphism, we will discuss both cases separately. For flat morphisms the pull-back is defined as in $[\mathbf{3 3}, 1.7]$, and for closed immersions one uses the deformation to the normal cone technique (see $[\mathbf{3 6}, 4.4 .1])$. Hence one can define the inverse image for a quasi-projective morphism using any factorization into a closed immersion followed by a flat morphism, and then proves that the result is independent of the chosen factorization. The main properties we need are those in the following theorem.

Theorem 1.38. Let $f: X \rightarrow Y$ be a quasi-projective morphism of regular schemes. Let $\varphi$ be a family of supports on $Y$, and let $\psi=f^{-1}(\varphi)$ be the corresponding family of supports on $X$.

(i) Assuming that $f=g \circ i=h \circ j$, where $i, j$ are closed immersions and $g, h$ are flat morphisms, we have

$$
i^{*} \circ g^{*}=j^{*} \circ h^{*} .
$$

Therefore, for all $p \geqslant 0$, there is a well-defined homomorphism of Chow groups

$$
f^{*}: \mathrm{CH}_{\varphi}^{p}(Y) \rightarrow \mathrm{CH}_{\psi}^{p}(X)
$$

(ii) The map $f^{*}$ induces a ring homomorphism

$$
f^{*}: \bigoplus_{p \geqslant 0} \mathrm{CH}^{p}(Y)_{\mathbb{Q}} \rightarrow \bigoplus_{p \geqslant 0} \mathrm{CH}^{p}(X)_{\mathbb{Q}} .
$$

(iii) The pull-back map $f^{*}: \mathrm{CH}^{p}(Y)_{\mathbb{Q}} \rightarrow \mathrm{CH}^{p}(X)_{\mathbb{Q}}$ corresponds via the isomorphisms

$$
\mathrm{CH}^{p}(Y)_{\mathbb{Q}} \cong H_{\mathcal{A}}^{2 p}(Y, \mathbb{Q}(p)) \text { and } \mathrm{CH}^{p}(X)_{\mathbb{Q}} \cong H_{\mathcal{A}}^{2 p}(X, \mathbb{Q}(p))
$$

to the pull-back defined in absolute cohomology.

(iv) If $g: Y \rightarrow Z$ is another morphism, then we have $(g \circ f)^{*}=f^{*} \circ g^{*}$.

To define the pull-back for $K_{1}$-chains, one observes first that the Gersten-Quillen spectral sequence is contravariant for flat morphisms. Moreover, in [34], there is a definition for a pull-back of $K_{1}$-chains for closed immersions using the deformation to the normal cone technique. The next result follows from [34] and [36, 4.4.2].

Theorem 1.39. Let $f: X \rightarrow Y$ be a quasi-projective morphism of regular schemes. Let $f=g \circ i$ be a factorization of $f$ into a closed immersion $i$ followed by a flat morphism $g$. Let $U \subseteq Y$ be an open subset and $Z \subseteq U$ a closed subset. Let us write $U^{\prime}=f^{-1}(U)$ and $Z^{\prime}=f^{-1}(Z)$. 
(i) For any $p \geqslant 0$, there is a homomorphism

$$
f^{*}=i^{*} \circ g^{*}: \mathrm{CH}_{Z}^{p-1, p}(U) \rightarrow \mathrm{CH}_{Z^{\prime}}^{p-1, p}\left(U^{\prime}\right)
$$

which does not depend on the factorization chosen.

(ii) Let us denote by $\delta$ the connection morphisms

$$
\begin{gathered}
\mathrm{CH}_{Z}^{p-1, p}(U) \stackrel{\delta}{\rightarrow} \mathrm{CH}_{\bar{Z} \backslash U}^{p}(Y), \\
\mathrm{CH}_{Z^{\prime}}^{p-1, p}\left(U^{\prime}\right) \stackrel{\delta}{\rightarrow} \mathrm{CH}_{\overline{Z^{\prime}} \backslash U^{\prime}}^{p}(X) .
\end{gathered}
$$

Then, we have $f^{*} \circ \delta=\delta \circ f^{*}$.

(iii) The induced map $f^{*}: \mathrm{CH}_{Z}^{p-1, p}(U)_{\mathbb{Q}} \rightarrow \mathrm{CH}_{Z^{\prime}}^{p-1, p}\left(U^{\prime}\right)_{\mathbb{Q}}$ corresponds via the isomorphisms

$$
\mathrm{CH}_{Z}^{p-1, p}(U)_{\mathbb{Q}} \cong H_{\mathcal{A}, Z}^{2 p-1}(U, \mathbb{Q}(p)) \quad \text { and } \quad \mathrm{CH}_{Z^{\prime}}^{p-1, p}\left(U^{\prime}\right)_{\mathbb{Q}} \cong H_{\mathcal{A}, Z^{\prime}}^{2 p-1}\left(U^{\prime}, \mathbb{Q}(p)\right)
$$

to the pull-back defined in absolute cohomology.

Proof. The definition of $i^{*}$ is given in $[\mathbf{3 6}, 4.4 .2]$. By definition, to show that $f^{*}$ is compatible with the connection morphisms and with the pull-back in absolute cohomology, it is enough to treat the flat morphisms and the closed immersions separately. For flat morphisms the result is clear. The compatibility with the connection morphisms for closed immersions is proven in $[\mathbf{3 6}, 4.4 .2]$. To prove the compatibility with the pullback in absolute cohomology, one observes that all the steps in the definition of $i^{*}$ are compatible with the isomorphism with absolute cohomology.

Push-forward and the projection formula

Let $f: X \rightarrow X^{\prime}$ be a proper morphism of regular, equidimensional, quasi-projective schemes in $\operatorname{ZAR}(S)$ of relative dimension $d$. Then, the Gersten-Quillen spectral sequence is covariant for proper morphisms (see [34]). In particular, for any closed subset $Y \subseteq X$, we have morphisms

$$
\begin{aligned}
f_{*}: \mathrm{CH}_{Y}^{p}(X) & \rightarrow \mathrm{CH}_{f(Y)}^{p-d}\left(X^{\prime}\right), \\
f_{*}: \mathrm{CH}_{Y}^{p-1, p}(X) & \rightarrow \mathrm{CH}_{f(Y)}^{p-d-1, p-d}\left(X^{\prime}\right) .
\end{aligned}
$$

These morphisms satisfy the following properties.

Theorem 1.40. Let $f: X \rightarrow X^{\prime}$ be a proper morphism of regular, equidimensional, quasi-projective schemes in $\operatorname{ZAR}(S)$, of relative dimension $d$.

(i) Let $Y$ be a closed subset of $X^{\prime}$. Let us write $U^{\prime}=X^{\prime} \backslash Y$ and $U=X \backslash f^{-1}(Y)$. Then, the following diagram commutes:

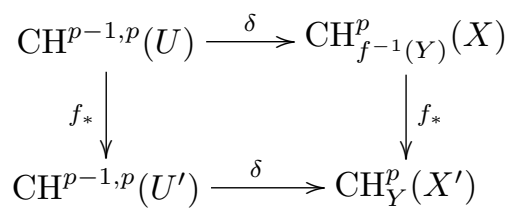


(ii) If $g: X^{\prime} \rightarrow X^{\prime \prime}$ is another proper morphism of regular, equidimensional, quasiprojective schemes in $\operatorname{ZAR}(S)$, we have

$$
(g \circ f)_{*}=g_{*} \circ f_{*} .
$$

(iii) The direct image of cycles and $K_{1}$-chains is compatible with the direct image in absolute cohomology.

(iv) For $\alpha \in \mathrm{CH}^{p}\left(X^{\prime}\right)$ and $\beta \in \mathrm{CH}^{q}(X)$ we have

$$
f_{*}\left(f^{*}(\alpha) \cdot \beta\right)=\alpha \cdot f_{*}(\beta) \in \mathrm{CH}^{p+q-d}\left(X^{\prime}\right)_{\mathbb{Q}} .
$$

Proof. The first and second statement follow from the covariance of the Gersten-Quillen spectral sequence. The third statement follows from the Riemann-Roch theorem, and the fourth statement follows from the third and the projection formula for absolute cohomology.

\subsection{Gillet cohomology and characteristic classes}

In this section we will recall some facts about characteristic classes from higher $K$ theory to a suitable cohomology theory. We will follow the axiomatic approach of [34]; hence, we will ask the cohomology theory to satisfy all the properties stated in [34]. Any such cohomology theory will be called a Gillet cohomology. Note however that the definition of Gillet cohomology given here is slightly different from the definition in [34].

\section{Gillet cohomologies}

We fix a field $k$. Let $\mathcal{V}$ be the category of smooth schemes over $k$ (recall that by scheme we mean a noetherian, separated scheme of finite Krull dimension). Let $\mathcal{V}_{*}$ be a category of schemes and proper morphisms that contains all closed immersions $Y \rightarrow X$ with $X$ in $\mathcal{V}$. Let $C$ be the big Zariski site of the category $\mathcal{V}$.

Definition 1.41. A Gillet cohomology is given by the following data.

(1) A graded complex of sheaves of abelian groups $\mathcal{G}^{*}(*)$ in the site $C$, together with a pairing in the derived category of graded complexes of abelian sheaves on $C$

$$
\mathcal{G}^{*}(*) \underset{\mathbb{Z}}{\stackrel{L}{\otimes}} \mathcal{G}^{*}(*) \rightarrow \mathcal{G}^{*}(*),
$$

which is associative, (graded-)commutative, and has a unit. Given such a complex $\mathcal{G}=\mathcal{G}^{*}(*)$, for each pair $(Y, X)$, with $X$ in $\mathcal{V}$ and $Y$ a closed subscheme of $X$, we define the cohomology groups of $X$ with coefficients in $\mathcal{G}$ and support in $Y$ by

$$
H_{Y}^{i}(X, \mathcal{G}(j))=\mathbb{H}_{Y}^{i}\left(X, \mathcal{G}^{*}(j)\right) .
$$

Since these cohomology groups are defined as the hypercohomology groups of a multiplicative Zariski sheaf, they satisfy the usual multiplicative and functorial properties (see [34, Definition 1.1]). 
(2) A homology theory, that is, a covariant functor from the category $\mathcal{V}_{*}$ to bigraded abelian groups

$$
X \mapsto \bigoplus_{\substack{i \geqslant 0 \\ j \in \mathbb{Z}}} H_{i}(X, \mathcal{G}(j))
$$

This homology-cohomology theory has to satisfy the properties (i)-(xi) of [34, Definition 1.2] with $d=2$. Since we are distinguishing between objects of $\mathcal{V}$ and of $\mathcal{V}_{*}$, we have to specify to which category the objects in question in properties (i)-(xi) belong: in (i), (ii), (viii), $X, Y \in \mathcal{V}_{*}$; in (iii), (v), $X \in \mathcal{V}, Y \in \mathcal{V}_{*}$; in (iv), $X \in \mathcal{V}_{*}$; in (vi), (vii), $X, Y \in \mathcal{V}$; and in (ix), (x), $X \in \mathcal{V}$. In addition to Gillet's axioms, following Beilinson [4], we will also assume

(xii) $H^{i}(\cdot, \mathcal{G}(j))=0$ for all $i<0$ and all $j \in \mathbb{Z}$.

Definition 1.42. A Gillet complex over $k$ is the graded complex of sheaves of abelian groups $\mathcal{G}=\mathcal{G}^{*}(*)$ of a Gillet cohomology.

Remark 1.43. The main discrepancy between the definition here and that of $[\mathbf{3 4}]$ is that the objects of $\mathcal{V}$ and of $\mathcal{V}_{*}$ are not the same. The reason for this is that we want a Gillet complex as a sheaf only over smooth schemes. On the other hand, we want to have cycle classes for arbitrary subschemes. Therefore, we need the homology also to be defined for singular schemes.

\section{Purity}

Property (vi) of the Gillet axioms includes the following purity condition: If $(X, Y)$ is a pair of schemes in $C$ with $Y$ a closed subscheme of $X$ of pure codimension $p$, then the natural map

$$
H^{i}(Y, \mathcal{G}(j)) \rightarrow H_{Y}^{i+2 p}(X, \mathcal{G}(j+p))
$$

is an isomorphism.

This purity condition, together with the vanishing assumption (xii), implies the following proposition.

Proposition 1.44. Let $\mathcal{G}$ be a Gillet complex over $k$, and $X$ a regular scheme in $\mathrm{ZAR}(\operatorname{Spec}(k))$. For any closed subset $Y$ of $X$ of codimension greater or equal to $p$, we then have

$$
H_{Y}^{i}(X, \mathcal{G}(p))=0
$$

for all $i<2 p$.

\section{Sheaf cohomology as generalized cohomology}

Usual sheaf cohomology of abelian sheaves can also be realized as generalized cohomology. The reader is referred to $[\mathbf{1 1}],[\mathbf{3 4}],[\mathbf{2 1}]$ and $[\mathbf{4 6}$, Appendix B] for details.

Given a cohomological complex of abelian groups $G^{*}$ indexed by non-positive integers, the Dold-Puppe functor associates to it a simplicial abelian group $K(G)$ pointed by 0 such that

$$
h^{-i}\left(G^{*}\right)=\pi_{i}(K(G), 0)
$$


Given a general cohomological complex $G$, we will denote by $\sigma_{p}$ the bête filtration (see $\S 2.4)$. Then, we can define an infinite loop spectrum

$$
K(G)_{N}=K\left(\sigma_{0}(G[N])\right) .
$$

We can recover all the cohomology groups of $G$ from the stable homotopy groups of this spectrum.

We can sheafify the above construction and then associate to each complex of abelian sheaves $\mathcal{G}$ an infinite loop spectrum of spaces $K(\mathcal{G})$. Moreover, we have the following proposition (see $[\mathbf{4 6}]$ ).

Proposition 1.45. Let $\mathcal{G}$ be a bounded below abelian sheaf over the site $C$. Then, we have for every $X$ in $C$,

$$
H_{s T}^{i}(X, K(\mathcal{G}))=H_{\mathrm{ZAR}}^{i}(X, \mathcal{G})
$$

\section{Characteristic classes}

To a Gillet cohomology theory, one can associate a theory of characteristic classes from higher $K$-theory (see $[\mathbf{4}, \mathbf{3 4}])$. These characteristic classes can be constructed on the level of spaces (see $[\mathbf{2 1}, \mathbf{3 4}]$ ). More specifically, we have the following result.

Theorem 1.46. Let $\bigoplus_{j \in \mathbb{Z}} \mathcal{G}^{*}(j)$ be a Gillet complex over $k$. Moreover, assume that the sheaves $\mathcal{G}^{i}(j)$ are injective for all $i, j$. Then, for every $j \geqslant 0$, there is a Chern class map of spaces

$$
c_{j}: \boldsymbol{K} \rightarrow K(\mathcal{G}(j)[2 j]) .
$$

These maps induce morphisms

$$
c_{j}: H_{s T}^{i}(X, \boldsymbol{K}) \rightarrow H_{s T}^{2 j+i}(X, K(\mathcal{G}(j)))=H_{\mathrm{ZAR}}^{2 j+i}(X, \mathcal{G}(j))
$$

for all spaces $X$.

Proof. The injectivity of $\mathcal{G}^{i}(j)$ implies that $\mathcal{G}^{*}(j)$ are pseudo-flasque complexes (see $\S 1.2$ ). This result is a particular case of [46, Theorem B.3.7].

This theorem implies in particular that there is a definition of Chern classes with support. That is, if $Y$ is a closed subscheme of $X$, then there are classes

$$
c_{j}^{Y}: K_{m}^{Y}(X) \rightarrow H_{Y}^{2 j-m}(X, \mathcal{G}(j)) .
$$

Moreover, these Chern classes are natural and satisfy the following proposition.

Proposition 1.47. Let $Y$ be a closed subset of $X$ and $U=X \backslash Y$. Then, the Chern classes form a morphism of exact sequences

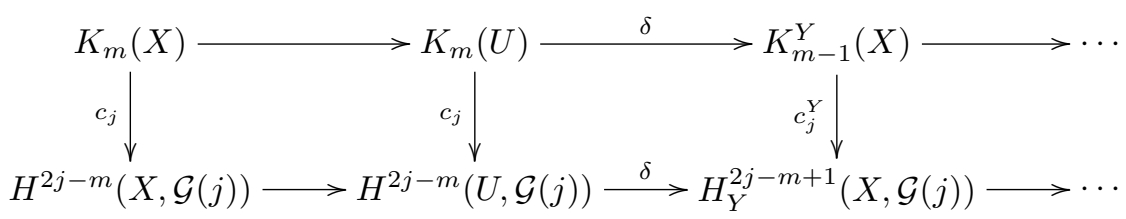




\section{Bloch-Ogus spectral sequence}

Since the set of axioms in [34] include in particular all the axioms of $[\mathbf{6}]$, we can form the Bloch-Ogus spectral sequence associated to the cohomology $\mathcal{G}$. Moreover, by the fact that characteristic classes are defined on the level of sheaves, characteristic classes induce morphisms from the Gersten-Quillen spectral sequence of $K$-theory to the Bloch-Ogus spectral sequence of $\mathcal{G}$-cohomology (see $[34, \S 3]$ ).

Let $x \in X^{(p)}$, and let $\iota: \overline{\{x\}} \rightarrow X$ be the inclusion. We will write

$$
\begin{aligned}
H^{i}(x, \mathcal{G}(j)) & =\underset{U}{\lim } H^{i}(\overline{\{x\}} \cap U, \mathcal{G}(j)), \\
H_{x}^{i}(X, \mathcal{G}(j)) & =\underset{U}{\lim } H_{\overline{\{x\}} \cap U}^{i}(X, \mathcal{G}(j)),
\end{aligned}
$$

where the limit is taken over all open sets $U$ of $X$ containing $x$. By the purity property of a Gillet cohomology, $\iota$ induces isomorphisms

$$
\iota !: H^{i}(x, \mathcal{G}(j)) \rightarrow H_{x}^{i+2 p}(X, \mathcal{G}(j+p)) .
$$

The Bloch-Ogus spectral sequence is a spectral sequence

$$
E_{r}^{p, q}(j) \Longrightarrow H^{p+q}(X, \mathcal{G}(j))
$$

with

$$
\begin{aligned}
E_{1}^{p, q}(j) & =\bigoplus_{x \in X^{(p)}} H_{x}^{p+q}(X, \mathcal{G}(j)) \\
& \cong \bigoplus_{x \in X^{(p)}} H^{q-p}(x, \mathcal{G}(j-p))
\end{aligned}
$$

Characteristic classes induce morphisms between the Gersten-Quillen spectral sequence and the Bloch-Ogus spectral sequence (see $[\mathbf{3 4}, \S 3]$ ).

Proposition 1.48. For each Chern class $c_{j}$ there is a morphism of spectral sequences

$$
\bigoplus_{x \in X^{(p)}} K_{-p-q}^{x}(X) \stackrel{\oplus c_{j}^{x}}{\longrightarrow} \bigoplus_{x \in X^{(p)}} H_{x}^{2 j+p+q}(X, \mathcal{G}(j)) .
$$

Moreover, this morphism is contravariant for flat morphisms.

We can form a commutative diagram

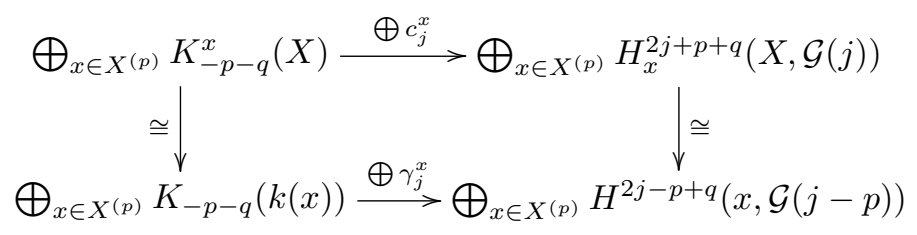

where the bottom arrow is determined as a consequence of the Riemann-Roch theorem without denominators (see [34, Theorem 3.9]). 
Lemma 1.49. Let $X$ be a smooth, noetherian $k$-scheme of finite Krull dimension. Let $x \in X^{(p)}$, and let $\eta_{x} \in H^{0}(x, \mathcal{G}(0))$ be the fundamental class. Then, there is a commutative diagram

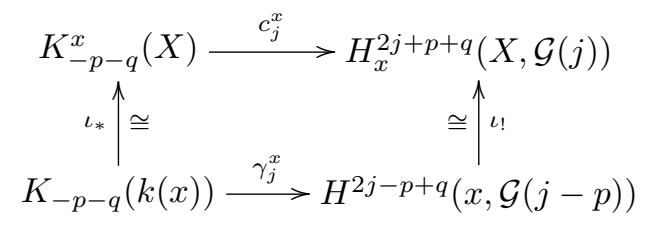

such that $\alpha \in K_{-p-q}(k(x))$ satisfies

$$
\gamma_{j}^{x}(\alpha)= \begin{cases}\frac{(-1)^{p}(j-1) !}{(j-p-1) !} c_{j-p}^{x}(\alpha), & \text { if } j>p, \\ \operatorname{rk}(\alpha) \eta_{x}, & \text { if } j=p .\end{cases}
$$

Classes for cycles and $K_{1}$-chains

For the construction of arithmetic Chow rings we are mainly interested in the induced classes of algebraic cycles and $K_{1}$-chains.

Definition 1.50. Let $x \in X^{(p)}$, and let $\iota: \overline{\{x\}} \rightarrow X$ be the inclusion. For $\alpha \in K_{0}(k(x))$, we will denote by $\operatorname{cl}_{\mathcal{G}}(\alpha)$ the class

$$
\operatorname{cl}_{\mathcal{G}}(\alpha)=\iota_{!}\left(\gamma_{p}^{x}(\alpha)\right)=\iota_{!}\left(\operatorname{rk}(\alpha) \eta_{x}\right) \in H_{x}^{2 p}(X, \mathcal{G}(p)) .
$$

Furthermore, for $f \in K_{1}(k(x))$, we will denote by $\operatorname{cl}_{\mathcal{G}}(f)$ the class

$$
\mathrm{cl}_{\mathcal{G}}(f)=\iota_{!}\left((-1)^{p} \gamma_{p+1}^{x}(f) / p !\right)=\iota_{!}\left(c_{1}^{x}(f)\right) \in H_{x}^{2 p+1}(X, \mathcal{G}(p+1)) .
$$

By linearity, we obtain well-defined morphisms

$$
\begin{aligned}
\mathrm{cl}_{\mathcal{G}}: R_{p}^{p}(X) & \rightarrow \bigoplus_{x \in X^{(p)}} H_{x}^{2 p}(X, \mathcal{G}(p)), \\
\mathrm{cl}_{\mathcal{G}}: R_{p}^{p-1}(X) & \rightarrow \bigoplus_{x \in X^{(p-1)}} H_{x}^{2 p-1}(X, \mathcal{G}(p)) .
\end{aligned}
$$

These classes should be interpreted as the Chern character. Namely, using the isomorphism between Chow groups and absolute cohomology, the classes of Definition 1.50 induce classes between absolute cohomology groups and the Gillet cohomology groups which agree with the Chern character.

Proposition 1.51. There are commutative diagrams

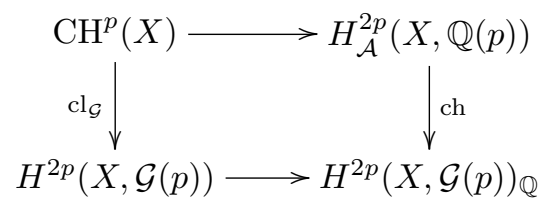


and

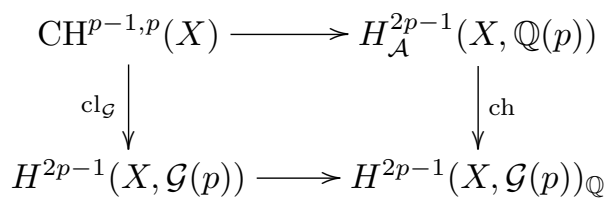

Proof. This follows from Lemma 1.49 and the power series expansion of the Chern character.

Basic properties of the morphism $\mathrm{cl}_{\mathcal{G}}$

The next result follows from the purity of a Gillet cohomology, Proposition 1.47 and Proposition 1.32.

\section{Proposition 1.52.}

(i) Let $y \in \bigoplus_{x \in X^{(p)}} K_{0}(k(x))$ be a $p$-codimensional cycle with $Y=\operatorname{supp} y$. Then, $\operatorname{cl}_{\mathcal{G}}(y)$ determines a well-defined class $\operatorname{cl}_{\mathcal{G}}(y) \in H_{Y}^{2 p}(X, \mathcal{G}(p))$.

(ii) For a $K_{1}$-chain $f \in R_{p}^{p-1}(X)$, put $y=\operatorname{div}(f), Y=\operatorname{supp} y$, and $U=X \backslash Y$. Then, $\operatorname{cl}_{\mathcal{G}}(f)$ determines a well-defined class $\operatorname{cl}_{\mathcal{G}}(f) \in H^{2 p-1}(U, \mathcal{G}(p))$.

(iii) With $f$ as in (ii), the equality

$$
\operatorname{cl}_{\mathcal{G}}(\operatorname{div}(f))=\delta \operatorname{cl}_{\mathcal{G}}(f) \in H_{Y}^{2 p}(X, \mathcal{G}(p))
$$

holds, where $\delta$ is the connection morphism

$$
\delta: H^{2 p-1}(U, \mathcal{G}(p)) \rightarrow H_{Y}^{2 p}(X, \mathcal{G}(p)) .
$$

(iv) If $h \in R_{p}^{p-2}(X)$ is a $K_{2}$-chain, then the class $\mathrm{cl}_{\mathcal{G}}(\mathrm{d} h)$ vanishes in the group $H^{2 p-1}(X, \mathcal{G}(p))$.

Corollary 1.53. Let $\varphi$ be any family of closed supports. Then, the morphisms $\mathrm{cl}_{\mathcal{G}}$ induce morphisms

$$
\begin{gathered}
\operatorname{cl}_{\mathcal{G}}: \mathrm{CH}_{\varphi}^{p}(X) \rightarrow H_{\varphi}^{2 p}(X, \mathcal{G}(p)), \\
\operatorname{cl}_{\mathcal{G}}: \mathrm{CH}_{\varphi}^{p-1, p}(X) \rightarrow H_{\varphi}^{2 p-1}(X, \mathcal{G}(p)) .
\end{gathered}
$$

Moreover, if $Y \subseteq X$ is a closed subset with $U=X \backslash Y$, then there is a morphism of exact sequences

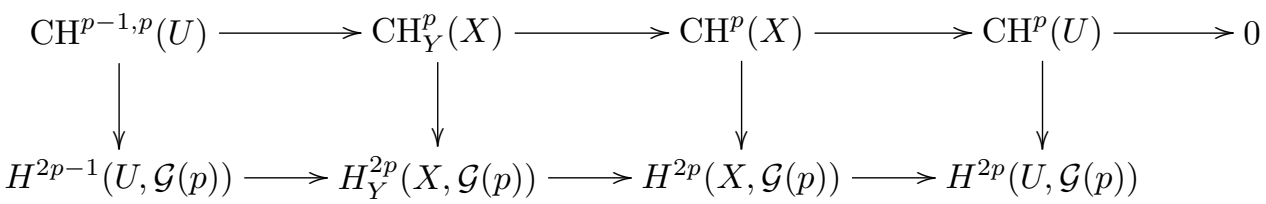


Product, pull-back and push-forward

Let us summarize the compatibility properties of characteristic classes from Chow groups with products, inverse images and direct images. Recall that in this section all schemes are defined over a field.

Proposition 1.54. The morphism

$$
\mathrm{cl}_{\mathcal{G}}: \bigoplus_{p \geqslant 0} \mathrm{CH}^{p}(X) \rightarrow \bigoplus_{p \geqslant 0} H^{2 p}(X, \mathcal{G}(p))
$$

is multiplicative.

Proposition 1.55. For a $K_{1}$-chain $f \in R_{p}^{p-1}(X)$, put $y=\operatorname{div}(f), Y=\operatorname{supp} y$, and $U=X \backslash Y$; for $z \in R_{q}^{q}(X)$, put $Z=\operatorname{supp} z$. Then, for any $K_{1}$-chain $g \in R_{p+q}^{p+q-1}(X)_{\mathbb{Q}}$ representing $[f \cdot z]$, the equality

$$
\operatorname{cl}_{\mathcal{G}}(g)=\operatorname{cl}_{\mathcal{G}}(f) \cdot \operatorname{cl}_{\mathcal{G}}(z) \in H_{U \cap Z}^{2 p+2 q-1}(X \backslash(Y \cap Z), \mathcal{G}(p+q))_{\mathbb{Q}}
$$

holds. Moreover, we have $\operatorname{cl}_{\mathcal{G}}(\operatorname{div}(g))=\operatorname{cl}_{\mathcal{G}}(\operatorname{div}(f)) \cdot \operatorname{cl}_{\mathcal{G}}(z)$.

Proposition 1.56. Let $f: X \rightarrow Y$ be a quasi-projective morphism of regular schemes. Let $\varphi$ be a family of supports on $Y$, and let $\psi=f^{-1}(\varphi)$ be the corresponding family of supports on $X$. Then, we have a commutative diagram

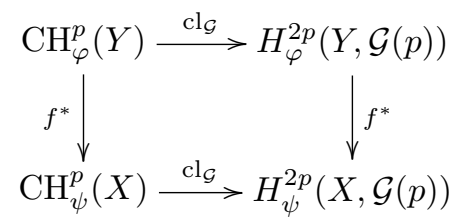

Furthermore, let $U \subseteq Y$ be an open subset and $Z \subseteq U$ a closed subset. Let us write $U^{\prime}=f^{-1}(U)$ and $Z^{\prime}=f^{-1}(Z)$. Then, we have a commutative diagram

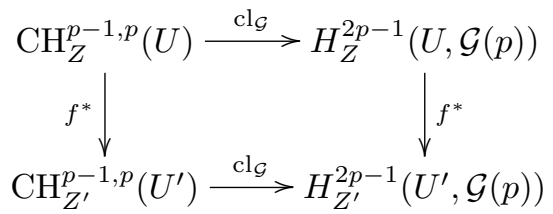

Proposition 1.57. Let $f: X \rightarrow X^{\prime}$ be a projective morphism of regular, equidimensional schemes of relative dimension $d$. Let $Y$ be a closed subset of $X$. Then, the diagrams

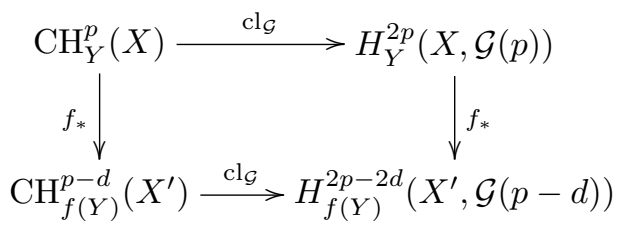


and

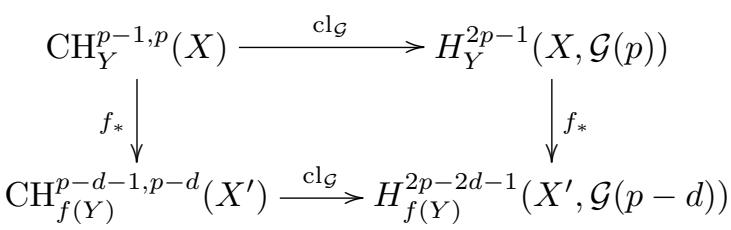

are commutative.

\section{Some topics from homological algebra}

\section{1. $k$-iterated complexes}

In this section we will introduce the notion of $k$-iterated complexes and discuss its relationship with $k$-complexes. Let $R$ be a commutative ring and $\mathcal{A}$ the category of $R$ modules.

$k$-complexes and $k$-iterated complexes

Definition 2.1. A $k$-complex $A=\left(A^{*, \ldots, *}, \mathrm{~d}_{1}, \ldots, \mathrm{d}_{k}\right)$ is a $k$-graded $R$-module $A$ together with $k$ endomorphisms $\mathrm{d}_{1}, \ldots, \mathrm{d}_{k}$ of multi-degrees

$$
(1,0, \ldots, 0), \ldots,(0, \ldots, 0,1),
$$

respectively, such that for all $i, j$

$$
\mathrm{d}_{i}^{2}=0, \quad \mathrm{~d}_{i} \mathrm{~d}_{j}=-\mathrm{d}_{j} \mathrm{~d}_{i} .
$$

We will denote by $k-\mathcal{C}^{+}(\mathcal{A})$ the category of $k$-complexes which are bounded from below, i.e. there is an integer $m$ such that $A^{n_{1}, \ldots, n_{k}}=0$, whenever $n_{i} \leqslant m(i=1, \ldots, k)$.

Definition 2.2. A $k$-iterated complex $A=\left(A^{*, \ldots, *}, \mathrm{~d}_{1}, \ldots, \mathrm{d}_{k}\right)$ is a $k$-graded $R$-module $A$ together with $k$ endomorphisms $\mathrm{d}_{1}, \ldots, \mathrm{d}_{k}$ of multi-degrees

$$
(1,0, \ldots, 0), \ldots,(0, \ldots, 0,1),
$$

respectively, such that for all $i, j$

$$
\mathrm{d}_{i}^{2}=0, \quad \mathrm{~d}_{i} \mathrm{~d}_{j}=\mathrm{d}_{j} \mathrm{~d}_{i} .
$$

We will denote by $\left(\mathcal{C}^{+}\right)^{k}(\mathcal{A})$ the category of $k$-iterated complexes which are bounded from below.

By convention, in the sequel all $k$-complexes and $k$-iterated complexes will be bounded from below, even if it is not stated explicitly.

Definition 2.3. Let $\mathcal{C}_{k}:\left(\mathcal{C}^{+}\right)^{k}(\mathcal{A}) \rightarrow k-\mathcal{C}^{+}(\mathcal{A})$ be the functor given by associating to a $k$-iterated complex $\left(A^{n_{1}, \ldots, n_{k}}, \mathrm{~d}_{1}, \mathrm{~d}_{2}, \ldots, \mathrm{d}_{k}\right)$ the $k$-complex

$$
\left(A^{n_{1}, \ldots, n_{k}}, \mathrm{~d}_{1},(-1)^{n_{1}} \mathrm{~d}_{2}, \ldots,(-1)^{n_{1}+\cdots+n_{k-1}} \mathrm{~d}_{k}\right) .
$$

This functor is an equivalence of categories. 
The simple complex

\section{Definition 2.4.}

(i) Let $A=\left(A^{n_{1}, \ldots, n_{k}}, \mathrm{~d}_{1}, \mathrm{~d}_{2}, \ldots, \mathrm{d}_{k}\right)$ be a $k$-complex. Then, the associated simple complex $s(A)$ is defined by

$$
s(A)^{n}=\bigoplus_{n_{1}+\cdots+n_{k}=n} A^{n_{1}, \ldots, n_{k}}, \quad \mathrm{~d}=\sum_{i=1}^{k} \mathrm{~d}_{i} .
$$

(ii) Let $A=\left(A^{n_{1}, \ldots, n_{k}}, \mathrm{~d}_{1}, \mathrm{~d}_{2}, \ldots, \mathrm{d}_{k}\right)$ be a $k$-iterated complex. Then, the associated simple complex is defined by

$$
s(A)=s\left(\mathcal{C}_{k}(A)\right)
$$

We have defined the simple complex of a $k$-iterated complex by contracting all the degrees into one degree. Sometimes it will be also useful to contract only some of the degrees making a partial simple complex.

Definition 2.5. Let $A=\left(A^{*, \ldots, *}, \mathrm{~d}_{A, 1}, \ldots, d_{A, k}\right)$ be a $k$-iterated complex with $k \geqslant 2$. We denote by $s_{j, j+1}(A)$ the $(k-1)$-iterated complex given by

$$
s_{j, j+1}(A)^{n_{1}, \ldots, n_{k-1}}=\bigoplus_{p+q=n_{j}} A^{n_{1}, \ldots, n_{j-1}, p, q, n_{j+1}, \ldots, n_{k-1}}
$$

with differentials

$$
\mathrm{d}_{i} x= \begin{cases}\mathrm{d}_{A, i} x, & \text { if } i<j, \\ \mathrm{~d}_{A, i} x+(-1)^{n_{i}} \mathrm{~d}_{A, i+1} x, & \text { if } i=j, \\ \mathrm{~d}_{A, i+1} x, & \text { if } i>j,\end{cases}
$$

where $x \in A^{n_{1}, \ldots, n_{j}, \ldots, n_{k}}$.

\section{Signs}

The simple complex of a $k$-iterated complex depends on the choice of an ordering of the degrees. If we choose a different ordering, we obtain a naturally isomorphic complex. In order to describe explicitly these isomorphisms it suffices to treat the case of the transposition of two adjacent degrees.

Definition 2.6. Let $T_{i, i+1}$ be the functor on $\left(\mathcal{C}^{+}\right)^{k}(\mathcal{A})$ to itself given, for $A \in\left(\mathcal{C}^{+}\right)^{k}(\mathcal{A})$, by

$$
T_{i, i+1}(A)^{n_{1}, \ldots, n_{i}, n_{i+1}, \ldots, n_{k}}=A^{n_{1}, \ldots, n_{i+1}, n_{i}, \ldots, n_{k}} .
$$

Proposition 2.7. Let $A$ be a $k$-iterated complex. The map

$$
s(A) \rightarrow s\left(T_{i, i+1}(A)\right)
$$

given by

$$
x \mapsto(-1)^{n_{i} n_{i+1}} x \quad\left(x \in A^{n_{1}, \ldots, n_{k}}\right)
$$

is an isomorphism of simple complexes. 
External product

Definition 2.8. Let $A$ be a $k$-iterated complex, and let $B$ be an $l$-iterated complex. Then, the external product $A \otimes B$ is the $(k+l)$-iterated complex with groups

$$
(A \otimes B)^{n_{1}, \ldots, n_{k+l}}=A^{n_{1}, \ldots, n_{k}} \otimes B^{n_{k+1}, \ldots, n_{k+l}},
$$

and differentials

$$
\mathrm{d}_{j}= \begin{cases}\mathrm{d}_{i} \otimes \mathrm{id}, & \text { if } i \leqslant k, \\ \mathrm{id} \otimes \mathrm{d}_{i-k}, & \text { if } i>k .\end{cases}
$$

The definitions of the functors $\mathcal{C}_{k}$ and of the simple complex of a $k$-iterated complex are chosen in order to have compatibility with the usual sign convention in the tensor product of complexes.

Lemma 2.9. Let $A$ be a $k$-iterated and $B$ be an $l$-iterated complex. The map

$$
s(A) \otimes s(B) \rightarrow s(A \otimes B)
$$

given by $a \otimes b \mapsto a \otimes b$ is an isomorphism of complexes.

Tensor product

Let us show how to define the tensor product in the category of 2-iterated complexes.

Definition 2.10. Let $A, B$ be a pair of 2-iterated complexes. Then, the tensor product $A \otimes B$ of $A$ and $B$ is the 2-iterated complex

$$
A \otimes B=s_{1,2} s_{3,4}\left(T_{2,3}(A \otimes B)\right) .
$$

Remark 2.11. This definition is justified by the fact that $A \otimes B$ satisfies the universal properties of a tensor product of $A$ and $B$ in the category of 2-iterated complexes. In particular, there are canonical isomorphisms $A \otimes B \cong B \otimes A$ and $A \otimes(B \otimes C) \cong(A \otimes B) \otimes C$; moreover, if $A, B, C, D$ are complexes of $R$-modules, then the identity map on the level of elements induces an isomorphism

$$
(A \otimes B) \otimes(C \otimes D) \cong(A \otimes C) \otimes(B \otimes D) .
$$

Examples

Let us discuss some fundamental examples of 2-iterated complexes.

Example 2.12. Let $f:\left(A^{*}, \mathrm{~d}_{A}\right) \rightarrow\left(B^{*}, \mathrm{~d}_{B}\right)$ be a morphism of complexes. Then, we can consider the 2 -iterated complex $\left(f^{* * *}, \mathrm{~d}^{\prime}, \mathrm{d}^{\prime \prime}\right)$ defined by

$$
f^{0, q}=A^{q}, \quad f^{1, q}=B^{q}, \quad \mathrm{~d}^{\prime}=f,\left.\quad \mathrm{~d}^{\prime \prime}\right|_{f^{0, *}}=\mathrm{d}_{A},\left.\quad \mathrm{~d}^{\prime \prime}\right|_{f^{1, *}}=\mathrm{d}_{B} .
$$

By abuse of notation we denote this 2-iterated complex by $f$. By definition, the simple complex $s(f)=s(A, B)$ of $f$ is given by $s(f)=s\left(\mathcal{C}_{2}\left(f^{*, *}\right)\right)$. Explicitly, we have

$$
s(f)^{n}=s(A, B)^{n}=A^{n} \oplus B^{n-1}, \quad \mathrm{~d}(a, b)=\left(\mathrm{d}_{A} a, f(a)-\mathrm{d}_{B} b\right) .
$$


Definition 2.13. Given a complex $\left(A^{*}, \mathrm{~d}\right)$, and an integer $k$, the shifted complex $A[k]$ is given by $A[k]^{n}=A^{n+k}$ with differential $(-1)^{k} \mathrm{~d}$.

Remark 2.14. Recall that the mapping cone of a morphism $f: A \rightarrow B$ of complexes is defined as

$$
\operatorname{cone}(f)^{n}=A^{n+1} \oplus B^{n}, \quad \mathrm{~d}(a, b)=\left(-\mathrm{d}_{A} a, f(a)+\mathrm{d}_{B} b\right) .
$$

Therefore, we have $s(f)=\operatorname{cone}(-f)[-1]$.

Example 2.15. Let $f: A \rightarrow B$ and $g: B \rightarrow C$ be morphisms of complexes with $g \circ f=0$. We may consider the diagram

$$
\xi=(A \stackrel{f}{\longrightarrow} B \stackrel{g}{\longrightarrow} C)
$$

as a 2-iterated complex $\left(\xi^{*, *}, \mathrm{~d}^{\prime}, \mathrm{d}^{\prime \prime}\right)$, where the groups are

$$
\xi^{0, q}=A^{q}, \quad \xi^{1, q}=B^{q}, \quad \xi^{2, q}=C^{q},
$$

the differential $\mathrm{d}^{\prime}$ is either $f$ or $g$ and the differential $\mathrm{d}^{\prime \prime}$ is the differential of the complexes $A, B$ or $C$. By abuse of notation, we denote this 2-iterated complex by $\xi$.

Proposition 2.16. With the notation of Example 2.15, let $\iota: A \rightarrow s(-g)$ be the morphism given by $\iota(a)=(f(a), 0)$. Then, there is a natural isomorphism of complexes

$$
s(\xi) \rightarrow s(A \stackrel{\iota}{\rightarrow} s(-g))
$$

given by $(a, b, c) \mapsto(a,(b, c))$.

The simple complex of a tensor product

For the rest of this section $f_{1}: A_{1} \rightarrow B_{1}$ and $f_{2}: A_{2} \rightarrow B_{2}$ will be morphisms of complexes. Considering these morphisms as 2-iterated complexes as in Example 2.12, we have by Definition 2.10

$$
f_{1} \otimes f_{2}=s_{1,2} s_{3,4}\left(T_{2,3}\left(f_{1} \otimes f_{2}\right)\right) .
$$

This 2-iterated complex is naturally identified with the 2-iterated complex associated to the diagram

$$
\xi=\left(A_{1} \otimes A_{2} \stackrel{\left(f_{1} \otimes \mathrm{id}, \mathrm{id} \otimes f_{2}\right)}{\longrightarrow} B_{1} \otimes A_{2} \oplus A_{1} \otimes B_{2} \stackrel{-\mathrm{id} \otimes f_{2}+f_{1} \otimes \mathrm{id}}{\longrightarrow} B_{1} \otimes B_{2}\right)
$$

as in Example 2.15.

Remark 2.19. Note that, if we consider $f_{1}: A_{1} \rightarrow B_{1}$ and $f_{2}: A_{2} \rightarrow B_{2}$ as morphisms and not as 2-iterated complexes, then $f_{1} \otimes f_{2}$ is the morphism $f_{1} \otimes f_{2}: A_{1} \otimes A_{2} \rightarrow B_{1} \otimes B_{2}$. It will be clear from the context which point of view is adopted in each case. If there is a danger of confusion, we will point out whether $f_{1} \otimes f_{2}$ is a 2-iterated complex or a morphism. 
Proposition 2.20. There is an isomorphism of complexes

$$
s\left(f_{1}\right) \otimes s\left(f_{2}\right) \rightarrow s\left(f_{1} \otimes f_{2}\right)
$$

given by

$$
\left(a_{1}, b_{1}\right) \otimes\left(a_{2}, b_{2}\right) \mapsto\left(a_{1} \otimes a_{2},\left(b_{1} \otimes a_{2},(-1)^{n} a_{1} \otimes b_{2}\right),(-1)^{n-1} b_{1} \otimes b_{2}\right),
$$

where $\left(a_{1}, b_{1}\right) \in s\left(f_{1}\right)^{n}$ and $\left(a_{2}, b_{2}\right) \in s\left(f_{2}\right)^{m}$.

Proof. By Lemma 2.9, we have an isomorphism $s\left(f_{1}\right) \otimes s\left(f_{2}\right) \cong s\left(f_{1} \otimes f_{2}\right)$. Since $s\left(f_{1} \otimes\right.$ $\left.f_{2}\right) \cong s\left(T_{2,3}\left(f_{1} \otimes f_{2}\right)\right)$ by Proposition 2.7 , and since $s\left(T_{2,3}\left(f_{1} \otimes f_{2}\right)\right)=s\left(s_{1,2} s_{3,4}\left(T_{2,3}\left(f_{1} \otimes\right.\right.\right.$ $\left.\left.f_{2}\right)\right)$ ), the existence of the claimed isomorphism follows by Definition 2.10 of $f_{1} \otimes f_{2}$.

Let us compute this isomorphism explicitly: for this we take $\left(a_{1}, b_{1}\right) \in s\left(f_{1}\right)^{n}$ and $\left(a_{2}, b_{2}\right) \in s\left(f_{2}\right)^{m}$. Viewing $a_{1}, b_{1}$, respectively $a_{2}, b_{2}$, as elements of the 2 -iterated complex $f_{1}$, respectively $f_{2}$, the elements $a_{1}, b_{1}, a_{2}, b_{2}$ have bidegree equal to $(0, n),(1, n-1)$, $(0, m),(1, m-1)$, respectively. Then, the element $\left(a_{1}, b_{1}\right) \otimes\left(a_{2}, b_{2}\right) \in s\left(f_{1}\right) \otimes s\left(f_{2}\right)$ is mapped to the element

$$
a_{1} \otimes a_{2}+b_{1} \otimes a_{2}+a_{1} \otimes b_{2}+b_{1} \otimes b_{2}
$$

of $s\left(f_{1} \otimes f_{2}\right)$, where the summands have the following 4-degree:

\begin{tabular}{|c|c|}
\hline element & 4-degree \\
\hline$a_{1} \otimes a_{2}$ & $(0, n, 0, m)$ \\
$b_{1} \otimes a_{2}$ & $(1, n-1,0, m)$ \\
$a_{1} \otimes b_{2}$ & $(0, n, 1, m-1)$ \\
$b_{1} \otimes b_{2}$ & $(1, n-1,1, m-1)$ \\
\hline
\end{tabular}

Applying $T_{2,3}$ to $f_{1} \otimes f_{2}$, the latter element is mapped to

$$
a_{1} \otimes a_{2}+b_{1} \otimes a_{2}+(-1)^{n} a_{1} \otimes b_{2}+(-1)^{n-1} b_{1} \otimes b_{2},
$$

which by the identification (2.17) is mapped to the element claimed in formula $(2.20)$.

\section{Commutativity of the external product}

The following observations will be needed in the discussion of commutativity and associativity of the product in relative cohomology defined in the next section.

Proposition 2.22. There is a commutative diagram of complexes

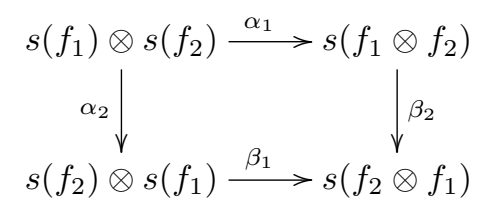

where $\alpha_{1}$ and $\beta_{1}$ are the isomorphisms determined in (2.20), $\alpha_{2}$ is given by

$$
\alpha_{2}\left(\left(a_{1}, b_{1}\right) \otimes\left(a_{2}, b_{2}\right)\right)=(-1)^{n m}\left(a_{2}, b_{2}\right) \otimes\left(a_{1}, b_{1}\right),
$$


and $\beta_{2}$ is given by

$$
\begin{aligned}
\beta_{2}\left(a_{1}\right. & \left.\otimes a_{2},\left(b_{1} \otimes a_{2}, a_{1} \otimes b_{2}\right), b_{1} \otimes b_{2}\right) \\
& =\left((-1)^{n m} a_{2} \otimes a_{1},\left((-1)^{n(m-1)} b_{2} \otimes a_{1},(-1)^{(n-1) m} a_{2} \otimes b_{1}\right),(-1)^{n m-n-m} b_{2} \otimes b_{1}\right)
\end{aligned}
$$

with $\operatorname{deg}\left(a_{1}\right)=n, \operatorname{deg}\left(b_{1}\right)=n-1, \operatorname{deg}\left(a_{2}\right)=m$ and $\operatorname{deg}\left(b_{2}\right)=m-1$.

Proof. First we note that $\beta_{2}$ is well defined by Proposition 2.20. The commutativity can now be checked directly. The signs in the definition of the morphisms $\alpha_{2}$ and $\beta_{2}$ are obtained as in the proof of Proposition 2.20.

Associativity of the external product

Furthermore, let $f_{3}: A_{3} \rightarrow B_{3}$ be a third morphism of complexes, and put $\phi_{1}=$ $f_{1} \otimes \mathrm{id} \otimes \mathrm{id}, \phi_{2}=\mathrm{id} \otimes f_{2} \otimes \mathrm{id}, \phi_{3}=\mathrm{id} \otimes \mathrm{id} \otimes f_{3}$. Then, the 2-iterated complexes $\left(f_{1} \otimes f_{2}\right) \otimes f_{3}$ and $f_{1} \otimes\left(f_{2} \otimes f_{3}\right)$ are both naturally identified with the 2 -iterated complex associated to the diagram

$$
\begin{aligned}
A_{1} \otimes A_{2} \otimes A_{3} & \stackrel{\delta_{1}}{\longrightarrow} B_{1} \otimes A_{2} \otimes A_{3} \oplus A_{1} \otimes B_{2} \otimes A_{3} \oplus A_{1} \otimes A_{2} \otimes B_{3} \\
& \stackrel{\delta_{2}}{\longrightarrow} B_{1} \otimes B_{2} \otimes A_{3} \oplus B_{1} \otimes A_{2} \otimes B_{3} \oplus A_{1} \otimes B_{2} \otimes B_{3} \\
& \stackrel{\delta_{3}}{\longrightarrow} B_{1} \otimes B_{2} \otimes B_{3},
\end{aligned}
$$

where

$$
\begin{aligned}
\delta_{1}(a) & =\left(\phi_{1} a, \phi_{2} a, \phi_{3} a\right), \\
\delta_{2}(a, b, c) & =\left(\phi_{1} b-\phi_{2} a, \phi_{1} c-\phi_{3} a, \phi_{2} c-\phi_{3} b\right), \\
\delta_{3}(a, b, c) & =\phi_{3} a-\phi_{2} b+\phi_{1} c .
\end{aligned}
$$

In the sequel we will denote any of the two 2-iterated complexes $\left(f_{1} \otimes f_{2}\right) \otimes f_{3}$ or $f_{1} \otimes$ $\left(f_{2} \otimes f_{3}\right)$ by $f_{1} \otimes f_{2} \otimes f_{3}$.

Proposition 2.26. There is a commutative diagram of complexes

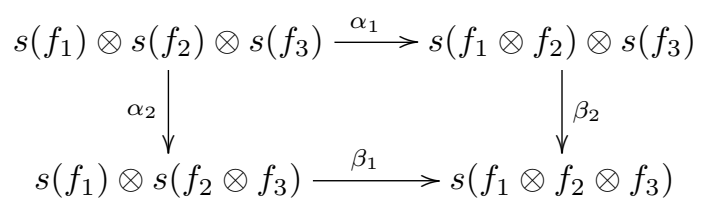

where $\alpha_{1}$ and $\alpha_{2}$ are isomorphisms induced by (2.20), $\beta_{1}$ is given by

$$
\begin{aligned}
\beta_{1}((a, b) \otimes(c,(d, e), f))=\left(a \otimes c,\left(b \otimes c,(-1)^{n} a \otimes d,(-1)^{n} a \otimes e\right),\right. & \left.\left((-1)^{n-1} b \otimes d,(-1)^{n-1} b \otimes e, a \otimes f\right), b \otimes f\right),
\end{aligned}
$$


with $n=\operatorname{deg}(a, b)$, and $\beta_{2}$ is given by

$$
\begin{aligned}
\beta_{2}((a,(b, c), d) \otimes(e, f))= & \left(a \otimes e,\left(b \otimes e, c \otimes e,(-1)^{n} a \otimes f\right),\right. \\
& \left.\left(d \otimes e,(-1)^{n-1} b \otimes f,(-1)^{n-1} c \otimes f\right),(-1)^{n-2} d \otimes f\right),
\end{aligned}
$$

with $n=\operatorname{deg}(a,(b, c), d)$.

Proof. The commutativity can be checked directly. The signs in the definition of the morphisms $\beta_{1}$ and $\beta_{2}$ are obtained as in the proof of Proposition 2.20.

\subsection{Relative cohomology groups}

Relative cohomology

Definition 2.29. Let $f: A \rightarrow B$ be a morphism of complexes. Then, the relative cohomology groups (of the pair $A, B$ ) are defined by

$$
H^{*}(A, B)=H^{*}(s(f)) .
$$

For a cycle $(a, b) \in s(f)^{n}$ we will denote by $[a, b]$ its class in $H^{n}(A, B)$. Observe that a cocycle of $s(f)$ is a pair $(a, b)$ with $\mathrm{d}_{A} a=0$ and $\mathrm{d}_{B} b=f(a)$. Moreover, the subspace of coboundaries is generated by elements of the form $\left(\mathrm{d}_{A} a, f(a)\right)$ and $\left(0, \mathrm{~d}_{B} b\right)$.

\section{Natural maps}

There are two natural maps relating the simple $s(f)$ of a morphism $f: A \rightarrow B$ with the underlying complexes $A, B$ :

$$
\alpha: s(f) \rightarrow A, \quad \text { given by }(a, b) \mapsto a,
$$

and

$$
\beta: B \rightarrow s(f)[1], \quad \text { given by } b \mapsto(0, b) \text {. }
$$

We will denote also by $\alpha$ and $\beta$ the corresponding morphisms for the cone given by the same formulae.

Distinguished exact triangles

Given a short exact sequence of complexes there are two possible choices for the connecting morphism; one is the opposite of the other. To choose the sign of the connecting morphism is equivalent to choose a set of distinguished exact triangles.

Given a morphism of complexes $f: A \rightarrow B$, one has chosen in [26, p. 269] the exact triangle

$$
A \stackrel{f}{\rightarrow} B \stackrel{\beta}{\rightarrow} \text { cone }(f) \stackrel{-\alpha}{\longrightarrow} A[1]
$$

as a distinguished exact triangle.

There are two basic principles that can be applied to obtain a distinguished exact triangle from another. The first one is that any exact triangle isomorphic (in the derived 
category) to a distinguished exact triangle is also distinguished. For instance, by means of the isomorphism cone $(f) \cong s(f)[1]$ given by mapping $(a, b)$ to $(-a, b)$, we find that the exact triangle

$$
A \stackrel{f}{\rightarrow} B \stackrel{\beta}{\rightarrow} s(f)[1] \stackrel{\alpha[1]}{\rightarrow} A[1]
$$

is also distinguished. As another example we can change the sign of two out of the three morphisms of a distinguished exact triangle, and the resulting exact triangle will again be distinguished; this results from the following isomorphism of exact triangles

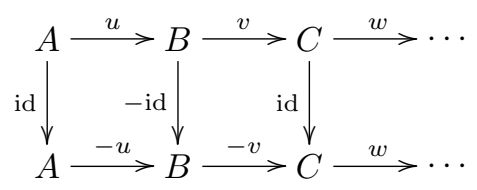

The second principle is that a distinguished exact triangle can be shifted by one and the resulting exact triangle is again exact provided that the sign of one of the morphisms is changed.

Applying these two principles to the distinguished exact triangle (2.29), we see that the exact triangles

$$
\begin{aligned}
s(f) \stackrel{\alpha}{\rightarrow} A \stackrel{f}{\rightarrow} B \stackrel{-\beta}{\rightarrow} \cdots, \\
s(-f) \stackrel{\alpha}{\rightarrow} A \stackrel{f}{\rightarrow} B \stackrel{\beta}{\rightarrow} \cdots
\end{aligned}
$$

are also distinguished. We note that these exact triangles are isomorphic by means of the isomorphism

$$
s(f) \rightarrow s(-f), \quad \text { given by }(a, b) \mapsto(a,-b) .
$$

Kernel, cokernel and simple complex of a morphism of complexes

Let

$$
0 \rightarrow A \stackrel{f}{\rightarrow} B \stackrel{g}{\rightarrow} C \rightarrow 0
$$

be a short exact sequence of complexes. The sign of the connecting homomorphism $\delta$ is determined by the isomorphisms (in the derived category) of exact triangles

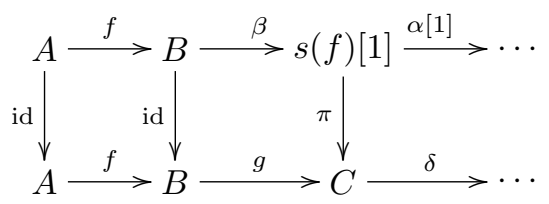

with $\pi(a, b)=g(b)$, and

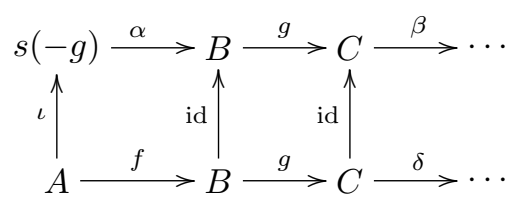

with $\iota(a)=(f(a), 0)$. 
Definition 2.37. The morphism $\pi$ will be called the simple-cokernel quasi-isomorphism, and the morphism $\iota$ will be called the kernel-simple quasi-isomorphism.

The sign of the connecting morphism determined by the above isomorphisms is given as follows. If $c$ is a cycle in $C^{n}$ satisfying $c=g(b)$ with $b \in B^{n}$, then we have

$$
\delta(c)=f^{-1}\left(\mathrm{~d}_{B} b\right) .
$$

Remark 2.39. Observe that the above connecting morphism is minus the connecting morphism considered in [17]. This forces us to adjust many of the signs.

The connecting morphism in relative cohomology

Since the exact triangle (2.31) is distinguished, we are led to the following definition.

Definition 2.40. Let $f: A \rightarrow B$ be a morphism of complexes. The connecting homomorphism in relative cohomology is the morphism

$$
\delta: H^{n-1}(B) \rightarrow H^{n}(A, B)
$$

induced by the natural map $-\beta$.

Split exact sequences

Let

$$
0 \rightarrow A \stackrel{f}{\rightarrow} B \stackrel{g}{\rightarrow} C \rightarrow 0
$$

be a short exact sequence of complexes. Assume that the short exact sequence

$$
0 \rightarrow A^{n} \stackrel{f}{\rightarrow} B^{n} \stackrel{g}{\rightarrow} C^{n} \rightarrow 0
$$

is split for all $n$. Thus there are sections $\sigma: C^{n} \rightarrow B^{n}$ such that $g \circ \sigma=\operatorname{id}_{C^{n}}$. These sections allow us to give a quasi-inverse of the simple-cokernel quasi-isomorphism $\pi$ and the kernel-simple quasi-isomorphism $\iota$.

Proposition 2.41. Let

$$
0 \rightarrow A \stackrel{f}{\rightarrow} B \stackrel{g}{\rightarrow} C \rightarrow 0
$$

be a short exact sequence as above such that for each $n$ there is a section $\sigma: C^{n} \rightarrow B^{n}$ of $g$. Then, we have the following statements.

(i) The map $\iota^{\prime}: C \rightarrow s(f)[1]$ given by

$$
\iota^{\prime}(c)=\left(f^{-1}\left(\mathrm{~d}_{B} \sigma(c)-\sigma\left(\mathrm{d}_{C} c\right)\right), \sigma(c)\right)
$$

is a morphism of complexes, and satisfies $\pi \circ \iota^{\prime}=\mathrm{id}_{C}$ and $\iota^{\prime} \circ \pi \sim \mathrm{id}_{s(f)[1]}$, where $\sim$ means homotopically equivalent.

(ii) The map $\pi^{\prime}: s(-g) \rightarrow A$ given by

$$
\pi^{\prime}(b, c)=f^{-1}\left(b-\sigma(g(b))-\sigma\left(\mathrm{d}_{C} c\right)+\mathrm{d}_{B} \sigma(c)\right)
$$

is a morphism of complexes satisfying $\pi^{\prime} \circ \iota=\mathrm{id}_{A}$, and $\iota \circ \pi^{\prime} \sim \mathrm{id}_{s(-g)}$, where ' $\sim$ ' means again homotopically equivalent. 


\section{Change of complex}

The following result is obvious. We quote it for future reference.

Proposition 2.42. Let $f: A \rightarrow B$ and $g: B \rightarrow C$ be morphisms of complexes such that $g$ is a quasi-isomorphism. Then, there is a natural quasi-isomorphism $s(f) \rightarrow s(g \circ f)$.

De Rham cohomology with support

Example 2.43. Let $X$ be a differentiable manifold and $Y$ a closed subset of $X$. Let us denote by $E(X)$ the complex of $\mathbb{C}$-valued differential forms and by $\sigma: E(X) \rightarrow E(X \backslash Y)$ the restriction morphism. Then, the relative de Rham cohomology groups of the pair $(X, X \backslash Y)$ are the cohomology groups of the simple $s(\sigma)$. By standard arguments in sheaf theory these groups can be naturally identified with the cohomology groups of the constant sheaf $\mathbb{C}$ with support in $Y$, denoted by $H_{Y}^{*}(X, \mathbb{C})$. Now let $Z$ be another closed subset of $X$ and $j$ the morphism

$$
j: E(X \backslash Y) \oplus E(X \backslash Z) \rightarrow E(X \backslash(Y \cup Z))
$$

given by $j(\omega, \eta)=-\omega+\eta$. The restriction of differential forms induces the Mayer-Vietoris sequence

$$
0 \rightarrow E(X \backslash(Y \cap Z)) \rightarrow E(X \backslash Y) \oplus E(X \backslash Z) \stackrel{j}{\rightarrow} E(X \backslash(Y \cup Z)) \rightarrow 0,
$$

where the first map assigns to $\omega$ the pair $(\omega, \omega)$. In this case the kernel-simple quasiisomorphism of Definition 2.37 is the quasi-isomorphism

$$
\iota: E(X \backslash(Y \cap Z)) \rightarrow s(-j)
$$

Using the quasi-isomorphism (2.43) together with Proposition 2.42, we obtain an isomorphism

$$
H_{Y \cap Z}^{n+m}(X, \mathbb{C}) \cong H^{n+m}(E(X), s(-j))
$$

Using partitions of unity and Proposition 2.41, one can construct a quasi-inverse of the kernel-simple quasi-isomorphism $\iota$, hence an inverse of the isomorphism (2.44).

\subsection{Products in relative cohomology}

External product

If $A$ and $B$ are complexes of $R$-modules, there is a well-defined external product

$$
H^{*}(A) \otimes H^{*}(B) \rightarrow H^{*}(A \otimes B)
$$

In particular, there is an external product in relative cohomology

$$
H^{n}\left(s\left(f_{1}\right)\right) \otimes H^{m}\left(s\left(f_{2}\right)\right) \rightarrow H^{n+m}\left(s\left(f_{1}\right) \otimes s\left(f_{2}\right)\right) .
$$


Proposition 2.46. The external product in relative cohomology can be identified with the pairing

$$
H^{n}\left(s\left(f_{1}\right)\right) \otimes H^{m}\left(s\left(f_{2}\right)\right) \rightarrow H^{n+m}\left(A_{1} \otimes A_{2}, s\left(\mathrm{id} \otimes f_{2}-f_{1} \otimes \mathrm{id}\right)\right)
$$

given by formula (2.20).

Proof. The result is a direct consequence of Proposition 2.20, the identification (2.17), and Proposition 2.16.

\section{Cup product}

We have defined an external product in relative cohomology. Moreover, if there is a product defined on the level of complexes, then we obtain a cup product in relative cohomology.

Theorem 2.47. Let

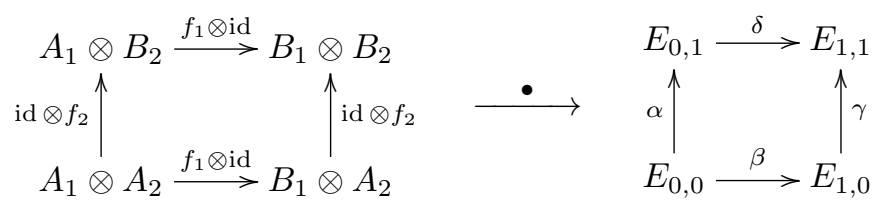

be a morphism of commutative diagrams in the category of complexes. Let $i: E_{0,0} \rightarrow$ $E_{1,0} \oplus E_{0,1}$, respectively $j: E_{1,0} \oplus E_{0,1} \rightarrow E_{1,1}$, be the morphism given by

$$
i(x)=(\beta(x), \alpha(x)), \quad \text { respectively } j(x, y)=-\gamma(x)+\delta(y) .
$$

Then, there is a pairing

$$
H^{n}\left(A_{1}, B_{1}\right) \otimes H^{m}\left(A_{2}, B_{2}\right) \stackrel{\bullet}{\rightarrow} H^{n+m}\left(E_{0,0}, s(-j)\right)
$$

given by

$$
\left[a_{1}, b_{1}\right] \bullet\left[a_{2}, b_{2}\right]=\left[a_{1} \bullet a_{2},\left(\left(b_{1} \bullet a_{2},(-1)^{n} a_{1} \bullet b_{2}\right),(-1)^{n-1} b_{1} \bullet b_{2}\right)\right] ;
$$

note that the map $E_{0,0} \rightarrow s(-j)$ is given by $x \mapsto(i(x), 0)$.

Proof. By Proposition 2.20 there is a pairing

$$
H^{n}\left(A_{1}, B_{1}\right) \otimes H^{m}\left(A_{2}, B_{2}\right) \rightarrow H^{n+m}(s(\xi)),
$$

where $\xi$ is given by (2.17). Let $\eta$ be the 2-iterated complex

$$
\eta=\left(E_{0,0} \stackrel{i}{\rightarrow} E_{1,0} \oplus E_{0,1} \stackrel{j}{\rightarrow} E_{1,1}\right) .
$$

From the data given in the assumptions, we obtain a morphism $\bullet$ of 2 -iterated complexes

$$
\bullet: \xi \rightarrow \eta
$$

hence we obtain a morphism $H^{n+m}(s(\xi)) \rightarrow H^{n+m}(s(\eta))$. By Proposition 2.16 the latter cohomology group is isomorphic to $H^{n+m}\left(E_{0,0}, s(-j)\right)$, which completes the proof. 
Remark 2.49. Letting $A_{1}=0$ in the above notation and observing that $H^{n}\left(0, B_{1}\right) \cong$ $H^{n-1}\left(B_{1}\right)$, we obtain from Theorem 2.47 with $E_{0,0}=0$ a pairing, also denoted by $\bullet$

$$
H^{n-1}\left(B_{1}\right) \otimes H^{m}\left(A_{2}, B_{2}\right) \stackrel{\bullet}{\rightarrow} H^{n+m-1}(s(-j))
$$

given by

$$
\left[b_{1}\right] \bullet\left[a_{2}, b_{2}\right]=\left[\left(b_{1} \bullet a_{2}, 0\right),(-1)^{n-1} b_{1} \bullet b_{2}\right] .
$$

Corollary 2.51. With the assumptions of Theorem 2.47 and Remark 2.49 we have a commutative diagram:

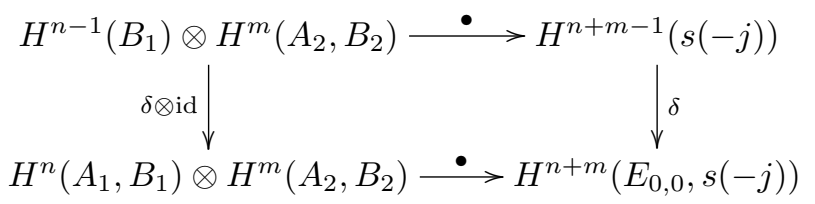

where $\delta$ is the connecting morphism in the long exact sequence of relative cohomology described in Definition 2.40.

The cup product in de Rham cohomology with support

Example 2.52. Recall the notation of Example 2.43. Let us illustrate how the formalism developed in Theorem 2.47 allows us to compute the product

$$
H_{Y}^{n}(X, \mathbb{C}) \otimes H_{Z}^{m}(X, \mathbb{C}) \rightarrow H_{Y \cap Z}^{n+m}(X, \mathbb{C})
$$

by means of differential forms. Let us write

$$
\begin{aligned}
& A_{1}=A_{2}=E_{0,0}=E(X), \quad B_{1}=E_{1,0}=E(X \backslash Y), \\
& B_{2}=E_{0,1}=E(X \backslash Z), \quad E_{1,1}=E(X \backslash(Y \cup Z)),
\end{aligned}
$$

and let $\bullet$ be the wedge product $\wedge$. Then, the assumptions of Theorem 2.47 are satisfied. Hence the wedge product induces a pairing

$$
H_{Y}^{n}(X, \mathbb{C}) \otimes H_{Z}^{m}(X, \mathbb{C}) \rightarrow H^{n+m}(E(X), s(-j))
$$

given, for appropriate cocycles $(\omega, \eta)$ and $\left(\omega^{\prime}, \eta^{\prime}\right)$, by

$$
\left(\omega \wedge \omega^{\prime},\left(\left(\eta \wedge \omega^{\prime},(-1)^{n} \omega \wedge \eta^{\prime}\right),(-1)^{n-1} \eta \wedge \eta^{\prime}\right)\right)
$$

this is a cocycle in $s(E(X), s(-j))$. Then, the desired pairing is defined by composing the pairing (2.52) with the inverse of the isomorphism (2.44). In particular, the cycle (2.53) represents a class in $H_{Y \cap Z}^{n+m}(X, \mathbb{C})$.

Using standard arguments from sheaf theory one can see that the product constructed above agrees with the sheaf theoretic product in cohomology with support (see, for example, [16, Proposition 2.5]). One can also compare this construction with the definition of the Loday product in $K$-theory with support given in $\S 1.2$. 


\subsection{Truncated relative cohomology groups}

Definition of truncated relative cohomology groups

Let $A=\left(A^{*}, \mathrm{~d}_{A}\right)$ be a complex of $R$-modules. We will denote by $\mathrm{Z}^{n}(A)$ the submodule of cocycles of $A^{n}$ and by $\widetilde{A}^{n}=A^{n} / \operatorname{Imd}_{A}$. For $a \in A^{n}$ we write $\widetilde{a}$ for its class in $\widetilde{A}^{n}$. Recall that the bête filtration $\sigma_{p} A$ of $A$ is given by

$$
\left(\sigma_{p} A\right)^{n}= \begin{cases}A^{n}, & \text { if } n \geqslant p, \\ 0, & \text { if } n<p .\end{cases}
$$

Definition 2.55. Let $f:\left(A^{*}, \mathrm{~d}_{A}\right) \rightarrow\left(B^{*}, \mathrm{~d}_{B}\right)$ be a morphism of complexes of $R$ modules. The truncated relative cohomology groups associated with $f$ are defined by

$$
\widehat{H}^{n}(A, B)=H^{n}\left(\sigma_{n} A, B\right) .
$$

In other words,

$$
\widehat{H}^{n}(A, B)=\left\{(a, \widetilde{b}) \in \mathrm{Z}^{n}(A) \oplus \widetilde{B}^{n-1} \mid f(a)=\mathrm{d}_{B} b\right\} .
$$

\section{Basic properties}

If $(a, b) \in \mathrm{Z}^{n}(s(A, B))$, we denote by $(a, \widetilde{b})$ its class in $\widehat{H}^{n}(A, B)$. The relative and truncated relative cohomology groups are $R$-modules in a natural way, and there are various natural maps relating them. The most important ones are the class map

$$
\mathrm{cl}: \widehat{H}^{n}(A, B) \rightarrow H^{n}(A, B), \quad \operatorname{cl}(a, \widetilde{b})=[a, b],
$$

and the cycle map

$$
\omega: \widehat{H}^{n}(A, B) \rightarrow \mathrm{Z}^{n}(A), \quad \omega(a, \widetilde{b})=a .
$$

We also recall the maps a and b, namely

$$
\mathrm{a}: \widetilde{A}^{n-1} \rightarrow \widehat{H}^{n}(A, B), \quad \mathrm{a}(\widetilde{a})=\left(-\mathrm{d}_{A} a,-\widetilde{f(a)}\right),
$$

and

$$
\mathrm{b}: H^{n-1}(B) \rightarrow \widehat{H}^{n}(A, B), \quad \mathrm{b}([b])=(0,-\widetilde{b}) .
$$

Denoting the induced morphism a $\left.\right|_{H^{n-1}(A)}: H^{n-1}(A) \rightarrow \widehat{H}^{n}(A, B)$ also by a, we observe for $[a] \in H^{n-1}(A)$ the relation

$$
\mathrm{a}([a])=\mathrm{b}(f(a))
$$

Finally, we note the following commutative diagram:

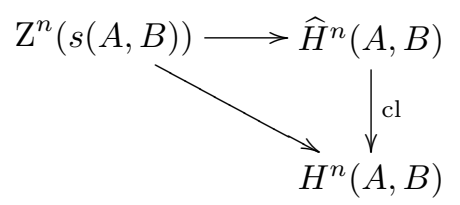


Remark 2.57. The signs of the maps $a$ and $b$ are the reverse of the signs in $[\mathbf{1 7}]$. This is due to the fact that the map b represents the connecting morphism in relative cohomology and we have changed the convention on the sign of the connecting morphism in order to be compatible with [26, p. 269]. The signs in the definition of the map a change accordingly.

For more details about truncated relative cohomology groups and the proof of the following proposition we refer to $[\mathbf{1 7}, \mathrm{p} .352]$.

Proposition 2.58. The following sequences are exact:

$$
\begin{aligned}
& H^{n-1}(A, B) \rightarrow \widetilde{A}^{n-1} \stackrel{\mathrm{a}}{\rightarrow} \widehat{H}^{n}(A, B) \stackrel{\mathrm{cl}}{\rightarrow} H^{n}(A, B) \rightarrow 0, \\
& 0 \rightarrow H^{n-1}(B) \stackrel{\mathrm{b}}{\rightarrow} \widehat{H}^{n}(A, B) \stackrel{\omega}{\rightarrow} \mathrm{Z}^{n}(A) \rightarrow H^{n}(B), \\
& H^{n-1}(A, B) \rightarrow H^{n-1}(A) \stackrel{\mathrm{a}}{\rightarrow} \widehat{H}^{n}(A, B) \stackrel{\mathrm{cl} \oplus \omega}{\rightarrow} H^{n}(A, B) \oplus \mathrm{Z}^{n}(A) \rightarrow H^{n}(A) \rightarrow 0 .
\end{aligned}
$$

Functorial properties

Let $f: A \rightarrow B$ and $f^{\prime}: A^{\prime} \rightarrow B^{\prime}$ be morphisms of complexes and let $g: f \rightarrow f^{\prime}$ be a morphism of the 2-iterated complexes $f, f^{\prime}$, i.e. $g$ is a pair of morphisms $\left(g_{A}, g_{B}\right)$ such that the diagram

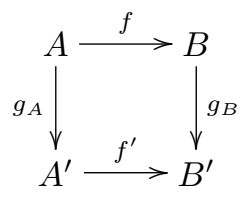

commutes.

Definition 2.62. The morphism induced (in truncated relative cohomology) by $g$ is the morphism

$$
\widehat{g}: \widehat{H}^{n}(A, B) \rightarrow \widehat{H}^{n}\left(A^{\prime}, B^{\prime}\right)
$$

defined by $\widehat{g}(a, \widetilde{b})=\left(g_{A}(a), \widetilde{g_{B}(b)}\right)$.

The exact sequence (2.59) implies the following corollary.

Corollary 2.63. Let $g$ be a morphism of 2-iterated complexes as in Definition 2.62 such that $g_{A}$ is an isomorphism and $g_{B}$ is a quasi-isomorphism. Then, the morphism $\widehat{g}$ induced by $g$ is an isomorphism.

This fundamental property of truncated relative cohomology groups will be of great use later. We also note that the requirement of $g_{A}$ being an isomorphism is essential. If we replace $A$ by a quasi-isomorphic complex, the truncated relative cohomology groups are no longer isomorphic. 


\subsection{Products in truncated relative cohomology}

\section{The *-product}

We now specialize the discussion about products in relative cohomology groups to the case of truncated relative cohomology. Let $f_{1}$ and $f_{2}$ be as in $\S 2.3$. Moreover, suppose that we have the morphism of commutative diagrams (2.47). Then, there is an induced morphism

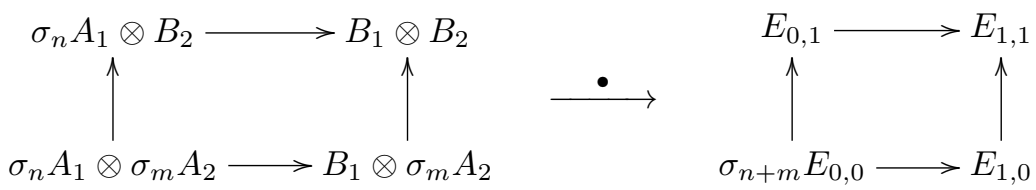

Definition 2.64. The $*$-product in truncated relative cohomology (induced by $\bullet$ ) is the pairing

$$
\widehat{H}^{n}\left(A_{1}, B_{1}\right) \otimes \widehat{H}^{m}\left(A_{2}, B_{2}\right) \stackrel{*}{\longrightarrow} \widehat{H}^{n+m}\left(E_{0,0}, s(-j)\right)
$$

provided by Theorem 2.47. In particular, for $\left(a_{1}, \widetilde{b}_{1}\right) \in \widehat{H}^{n}\left(A_{1}, B_{1}\right)$ and $\left(a_{2}, \widetilde{b}_{2}\right) \in$ $\widehat{H}^{m}\left(A_{2}, B_{2}\right)$, it is given by

$$
\left(a_{1}, \widetilde{b}_{1}\right) *\left(a_{2}, \widetilde{b}_{2}\right)=\left(a_{1} \bullet a_{2},\left(\left(b_{1} \bullet a_{2},(-1)^{n} a_{1} \bullet b_{2}\right),(-1)^{n-1} b_{1} \bullet b_{2}\right)^{\sim}\right) .
$$

Notice that, since the $*$-product is induced by a pairing of complexes, the right-hand side of the above equation is independent of the choice of representatives $b_{1}$ and $b_{2}$ of $\widetilde{b}_{1}$ and $\widetilde{b}_{2}$.

\section{Properties of the *-product}

We now summarize the basic properties of the $*$-product.

Proposition 2.66. With the above notation, there are commutative diagrams

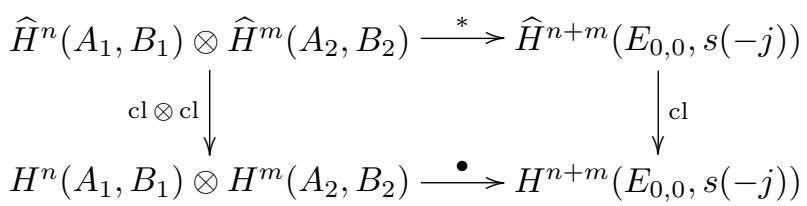

and

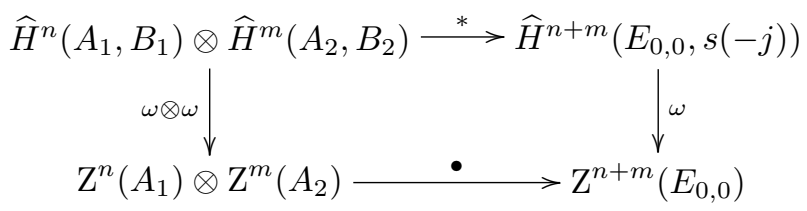


Proposition 2.67. With the above notation we have the following statements.

(i) $\mathrm{b}\left(H\left(B_{1}\right)\right) * \mathrm{a}(\widetilde{A} 2)=0$.

(ii) If $\left[b_{1}\right] \in H^{n-1}\left(B_{1}\right)$ and $g=\left(a_{2}, \widetilde{b}_{2}\right) \in \widehat{H}^{m}\left(A_{2}, B_{2}\right)$, then the following equality holds in the group $\widehat{H}^{n+m}\left(E_{0,0}, s(-j)\right)$ :

$$
\mathrm{b}\left(\left[b_{1}\right]\right) * g=\mathrm{b}\left(\left[b_{1}\right] \bullet \mathrm{cl}(g)\right)
$$

with $\left[b_{1}\right] \bullet \operatorname{cl}(g) \in H^{n+m-1}(s(-j))$ given by the pairing (2.49).

(iii) If $\widetilde{a}_{1} \in \widetilde{A}_{1}^{n-1}$ and $g=\left(a_{2}, \widetilde{b}_{2}\right) \in \widehat{H}^{m}\left(A_{2}, B_{2}\right)$, then the following equality holds in the group $\widehat{H}^{n+m}\left(E_{0,0}, s(-j)\right)$ :

$$
\mathrm{a}\left(\widetilde{a}_{1}\right) * g=\mathrm{a}\left(\widetilde{a_{1} \bullet \omega(g)}\right)
$$

with $\widetilde{a_{1} \bullet \omega(g)} \in \widetilde{E}_{0,0}^{n+m-1}$ given by the pairing $A_{1} \otimes A_{2} \stackrel{\bullet}{\rightarrow} E_{0,0}$.

(iv) If $\left[a_{1}\right] \in H^{n-1}\left(A_{1}\right)$, and $g=\left(a_{2}, \widetilde{b}_{2}\right) \in \widehat{H}^{m}\left(A_{2}, B_{2}\right)$, then, again in the group $\widehat{H}^{n+m}\left(E_{0,0}, s(-j)\right)$, we have the equality

$$
\mathrm{a}\left(\left[a_{1}\right]\right) * g=\mathrm{a}\left(\left[a_{1}\right] \bullet\left[a_{2}\right]\right) ;
$$

here $\left[a_{2}\right]$ is the class of $a_{2}$ in $H^{m}\left(A_{2}\right)$.

Proof. (i) For $\left[b_{1}\right] \in H^{n-1}\left(B_{1}\right)$ and $\widetilde{a}_{2} \in \widetilde{A}_{2}$, we have

$$
\begin{aligned}
\mathrm{b}\left(\left[b_{1}\right]\right) * \mathrm{a}\left(\widetilde{a}_{2}\right) & =\left(0,-\widetilde{b}_{1}\right) *\left(-\mathrm{d}_{A_{2}} a_{2},-\widetilde{f_{2}\left(a_{2}\right)}\right) \\
& =\left(0,\left(\left(b_{1} \bullet \mathrm{d}_{A_{2}} a_{2}, 0\right),(-1)^{n-1} b_{1} \bullet f_{2}\left(a_{2}\right)\right)^{\sim}\right) .
\end{aligned}
$$

Since $b_{1}$ is a cycle, we have

$$
(-1)^{n-1} \mathrm{~d}\left(\left(b_{1} \bullet a_{2}, 0\right), 0\right)=\left(\left(b_{1} \bullet \mathrm{d}_{A_{2}} a_{2}, 0\right),(-1)^{n-1} b_{1} \bullet f_{2}\left(a_{2}\right)\right),
$$

which immediately proves the first statement.

(ii) The formula we seek follows by a straightforward computation.

(iv) For a cycle $a_{1} \in A_{1}^{n-1}$, we have

$$
\begin{aligned}
\mathrm{a}\left(\widetilde{a}_{1}\right) *\left(a_{2}, \widetilde{b}_{2}\right) & =\left(0,-\widetilde{f_{1}\left(a_{1}\right)}\right) *\left(a_{2}, \widetilde{b}_{2}\right) \\
& =\left(0,-\left(\left(f_{1}\left(a_{1}\right) \bullet a_{2}, 0\right),(-1)^{n-1} f_{1}\left(a_{1}\right) \bullet b_{2}\right)^{\sim}\right) .
\end{aligned}
$$

Now we note

$$
\mathrm{d}\left(\left(0, a_{1} \bullet b_{2}\right), 0\right)=\left(\left(0, \mathrm{~d}_{A_{1}} a_{1} \bullet b_{2}+(-1)^{n-1} a_{1} \bullet \mathrm{d}_{B_{2}} b_{2}\right),-f_{1}\left(a_{1}\right) \bullet b_{2}\right),
$$

hence

$$
(-1)^{n} \mathrm{~d}\left(\left(0, a_{1} \bullet b_{2}\right), 0\right)=\left(\left(0,-a_{1} \bullet f_{2}\left(a_{2}\right)\right),(-1)^{n-1} f_{1}\left(a_{1}\right) \bullet b_{2}\right) .
$$

This immediately leads to

$$
\mathrm{a}\left(\widetilde{a}_{1}\right) *\left(a_{2}, \widetilde{b}_{2}\right)=\left(0,-\left(\left(f_{1}\left(a_{1}\right) \bullet a_{2}, a_{1} \bullet f_{2}\left(a_{2}\right)\right), 0\right)^{\sim}\right)=\mathrm{a}\left(\widetilde{a_{1} \bullet a_{2}}\right),
$$

which proves the claim.

(iii) Since $\omega(g)=a_{2}$, the equality follows immediately from the proof given for part (iv). 


\section{Example}

The following gives an example of the formalism developed for truncated cohomology.

Let $A$ be an associative, graded commutative, differential algebra over $R$, and $B$ a graded commutative, differential algebra over $R$, which is associative up to homotopy, i.e. there exist morphisms

$$
h: B^{n} \otimes B^{m} \otimes B^{l} \rightarrow B^{n+m+l-1}
$$

satisfying

$$
\left(b_{1} b_{2}\right) b_{3}-b_{1}\left(b_{2} b_{3}\right)=\mathrm{d} h\left(b_{1} \otimes b_{2} \otimes b_{3}\right)+h \mathrm{~d}\left(b_{1} \otimes b_{2} \otimes b_{3}\right) .
$$

Furthermore, let $f: A \rightarrow B$ be a morphism of graded differential algebras satisfying

$$
h\left(f\left(a_{1}\right) \otimes f\left(a_{2}\right) \otimes f\left(a_{3}\right)\right)=0
$$

for all elements $a_{1}, a_{2}, a_{3}$ in $A$.

Proposition 2.68. Let $A, B, f: A \rightarrow B$ be as above. Then, we have the following statements.

(i) The truncated cohomology groups $\widehat{H}^{*}(A, B)$ have a natural structure of an associative, graded commutative algebra.

(ii) In the particular case assumed we have $\mathrm{b}\left(H^{*}(B)\right) * \widehat{H}^{*}(A, B)=0$.

(iii) The class map cl : $\widehat{H}^{*}(A, B) \rightarrow H^{*}(A, B)$ is a morphism of algebras.

(iv) The cycle map $\omega: \widehat{H}^{*}(A, B) \rightarrow \mathrm{Z}^{*}(A)$ is a morphism of algebras.

Proof. The key point of the proof is to show (i). Then (iii), (iv) will follow from Proposition 2.16; part (ii) is a direct consequence of the explicit description of the $*$-product under the above assumptions and will be shown at the end of the proof.

By assumption, we have a morphism of commutative diagrams

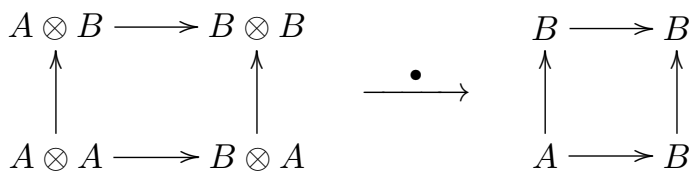

where $\bullet$ is given by the underlying algebra structure. Therefore, if $j: B \oplus B \rightarrow B$ is the morphism given by $j(x, y)=-x+y$, we obtain a pairing

$$
\widehat{H}^{n}(A, B) \otimes \widehat{H}^{m}(A, B) \stackrel{*}{\rightarrow} \widehat{H}^{n+m}(A, s(-j)) .
$$

Now consider the short exact sequence

$$
0 \longrightarrow B \stackrel{i}{\longrightarrow} B \oplus B \stackrel{j}{\longrightarrow} B \longrightarrow 0
$$


where the morphism $i: B \rightarrow B \oplus B$ is given by $i(x)=(x, x)$. By the kernelsimple quasi-isomorphism (see Definition 2.37), $i$ induces a quasi-isomorphism between $B$ and $s(-j)$. By means of Corollary 2.63 we then obtain a natural isomorphism $\widehat{H}^{n+m}(A, s(-j)) \cong \widehat{H}^{n+m}(A, B)$. Therefore, the *-product defines an inner product on $\widehat{H}^{*}(A, B)$.

We now give an explicit formula for the $*$-product. Since the exact sequence $(2.68)$ is split, we easily find an explicit quasi-inverse for the quasi-isomorphism induced by $i$. Namely, by applying Proposition 2.41, we can choose a quasi-inverse from $s(-j)$ to $B$ by the morphism given by

$$
((x, y), z) \mapsto x
$$

With this choice we derive from (2.64) the formula

$$
\left(a_{1}, \widetilde{b}_{1}\right) *\left(a_{2}, \widetilde{b}_{2}\right)=\left(a_{1} a_{2}, \widetilde{b_{1} f\left(a_{2}\right)}\right) .
$$

By Corollary 2.63 this product does not depend on the choice of the quasi-inverse.

We now show that the $*$-product is graded commutative. To do so, let $\left(a_{1}, \widetilde{b}_{1}\right) \in$ $\widehat{H}^{n}(A, B),\left(a_{2}, \widetilde{b}_{2}\right) \in \widehat{H}^{m}(A, B)$, and let $\left(a_{1}, b_{1}\right),\left(a_{2}, b_{2}\right)$ be respective representatives. Then, the difference

$$
\left(a_{1}, \widetilde{b}_{1}\right) *\left(a_{2}, \widetilde{b}_{2}\right)-(-1)^{n m}\left(a_{2}, \tilde{b}_{2}\right) *\left(a_{1}, \widetilde{b}_{1}\right)
$$

is represented by

$$
\left(a_{1} a_{2}-(-1)^{n m} a_{2} a_{1}, b_{1} f\left(a_{2}\right)-(-1)^{n m} b_{2} f\left(a_{1}\right)\right)=\left(0, b_{1} f\left(a_{2}\right)-(-1)^{n} f\left(a_{1}\right) b_{2}\right) .
$$

Since $\mathrm{d} b_{1}=f\left(a_{1}\right)$ and $\mathrm{d} b_{2}=f\left(a_{2}\right)$, we obtain

$$
(-1)^{n-1} \mathrm{~d}\left(b_{1} b_{2}\right)=b_{1} f\left(a_{2}\right)-(-1)^{n} f\left(a_{1}\right) b_{2} .
$$

This shows

$$
\left(a_{1}, \widetilde{b}_{1}\right) *\left(a_{2}, \widetilde{b}_{2}\right)=(-1)^{n m}\left(a_{2}, \widetilde{b}_{2}\right) *\left(a_{1}, \widetilde{b}_{1}\right) .
$$

Finally, we show that the $*$-product is associative. For this purpose, let $\left(a_{3}, \widetilde{b}_{3}\right) \in$ $\widehat{H}^{l}(A, B)$ be a third element. Consider the difference

$$
\left(\left(a_{1}, \widetilde{b}_{1}\right) *\left(a_{2}, \widetilde{b}_{2}\right)\right) *\left(a_{3}, \widetilde{b}_{3}\right)-\left(a_{1}, \widetilde{b}_{1}\right) *\left(\left(a_{2}, \widetilde{b}_{2}\right) *\left(a_{3}, \widetilde{b}_{3}\right)\right),
$$

which is represented by

$$
\left(\left(a_{1} a_{2}\right) a_{3},\left(b_{1} f\left(a_{2}\right)\right) f\left(a_{3}\right)\right)-\left(a_{1}\left(a_{2} a_{3}\right), b_{1}\left(f\left(a_{2}\right) f\left(a_{3}\right)\right)\right) .
$$

We have to show that the class of the latter difference in $\widehat{H}^{n+m+l}(A, B)$ is zero. By the associativity of $A$, we have $\left(a_{1} a_{2}\right) a_{3}=a_{1}\left(a_{2} a_{3}\right)$. Moreover, by the associativity of $B$ up to homotopy we have

$$
\left(b_{1} f\left(a_{2}\right)\right) f\left(a_{3}\right)-b_{1}\left(f\left(a_{2}\right) f\left(a_{3}\right)\right)=\mathrm{d} h\left(b_{1} \otimes f\left(a_{2}\right) \otimes f\left(a_{3}\right)\right)+h \mathrm{~d}\left(b_{1} \otimes f\left(a_{2}\right) \otimes f\left(a_{3}\right)\right) .
$$


Since $f\left(a_{2}\right)$ and $f\left(a_{3}\right)$ are cycles, and since $\mathrm{d} b_{1}=f\left(a_{1}\right)$, we obtain by assumption

$$
h \mathrm{~d}\left(b_{1} \otimes f\left(a_{2}\right) \otimes f\left(a_{3}\right)\right)=h\left(f\left(a_{1}\right) \otimes f\left(a_{2}\right) \otimes f\left(a_{3}\right)\right)=0 .
$$

From this the associativity of the $*$-product follows.

Statement (ii) is obvious from the explicit formula (2.69) given for the $*$-product.

Remark 2.71. There are many discrepancies in the signs between this paper and the previous papers $[\mathbf{1 6}, \mathbf{1 7}]$. The main source for these discrepancies is that the convention for the sign of the connecting morphism in this paper is minus the connecting morphism in $[\mathbf{1 7}]$. In particular, compare formula $(2.37)$ with $[\mathbf{1 7}, \S 2]$. But there are also some differences in the sign of the product.

\section{Green objects}

The aim of this section is to develop an abstract theory of Green objects. It is a generalization of $[\mathbf{1 7}]$ and some arguments, like the proof of the associativity and the commutativity of $[\mathbf{1 7}]$, carry over to this abstract setting directly.

\section{1. $\mathcal{G}$-complexes}

\section{The Mayer-Vietoris principle}

Definition 3.1. Let $X$ be a topological space. We say that a sheaf $\mathcal{F}$ is a totally acyclic sheaf, if the restriction $\left.\mathcal{F}\right|_{U}$ of $\mathcal{F}$ to $U$ is acyclic for all open subsets $U$ of $X$.

We note that for instance every flasque or fine sheaf on $X$ is totally acyclic.

Definition 3.2. We say that a presheaf $\mathcal{F}$ on $X$ satisfies the Mayer-Vietoris principle, if the sequence

$$
0 \rightarrow \mathcal{F}(U \cup V) \stackrel{i}{\rightarrow} \mathcal{F}(U) \oplus \mathcal{F}(V) \stackrel{j}{\rightarrow} \mathcal{F}(U \cap V) \rightarrow 0
$$

with $i(\eta)=(\eta, \eta)$ and $j(\omega, \eta)=-\omega+\eta$ is exact for any pair of open subsets $U$ and $V$ of $X$.

Proposition 3.3. Let $X$ be a noetherian space. Then, a presheaf $\mathcal{F}$ on $X$ satisfies the Mayer-Vietoris principle, if and only if it is a totally acyclic sheaf.

Proof. Since $X$ is noetherian, the exactness of

$$
0 \rightarrow \mathcal{F}(U \cup V) \stackrel{i}{\rightarrow} \mathcal{F}(U) \oplus \mathcal{F}(V) \stackrel{j}{\rightarrow} \mathcal{F}(U \cap V)
$$

is equivalent to the fact that $\mathcal{F}$ is a sheaf. If $\mathcal{F}$ is totally acyclic, then the map $j$ is surjective. Hence $\mathcal{F}$ satisfies the Mayer-Vietoris principle. Conversely, if $\mathcal{F}$ satisfies the Mayer-Vietoris principle, one can use induction on the number of open sets to show that, for any finite open covering $\mathfrak{U}$ of $X$, the Čech cohomology group $H^{1}(\mathfrak{U}, \mathcal{F})$ vanishes. 
This implies that, if $\mathcal{F}^{\prime}$ satisfies the Mayer-Vietoris principle, then for any short exact sequence

$$
0 \rightarrow \mathcal{F}^{\prime} \rightarrow \mathcal{F} \rightarrow \mathcal{F}^{\prime \prime} \rightarrow 0
$$

and any open set $U$, the sequence

$$
0 \rightarrow \mathcal{F}^{\prime}(U) \rightarrow \mathcal{F}(U) \rightarrow \mathcal{F}^{\prime \prime}(U) \rightarrow 0
$$

is exact. Then, the Nine Lemma implies that, if the sheaves $\mathcal{F}^{\prime}$ and $\mathcal{F}$ in an exact sequence as above satisfy the Mayer-Vietoris principle, then $\mathcal{F}^{\prime \prime}$ also does. One finally concludes the proof as in $[\mathbf{4 2}$, III 2.5].

\section{Definition of $\mathcal{G}$-complexes}

In order to define arithmetic Chow groups with Green objects in a certain cohomology theory, the main property we have to require of the cohomology theory is that it receives characteristic classes from $K$-theory (at least from $K_{0}$ and $K_{1}$ ) with natural properties. Since the arithmetic Chow groups will depend on the choice of the complex used to compute the cohomology, it might be more important to have a particular property of this complex than to have all the properties of a Gillet cohomology. Thus, in order to have characteristic classes, but also to retain flexibility in the choice of the complexes, we will ask only that the cohomology theory factors through a Gillet cohomology. Thus, we fix a field $k$ and an auxiliary Gillet complex $\mathcal{G}=\mathcal{G}^{*}(*)$ for regular schemes of finite type over $k$. Furthermore, until the discussion of the functoriality, we also fix a regular separated scheme $X$ of finite type over $k$.

Definition 3.4. A $\mathcal{G}$-complex over $X$ is a graded complex $\mathcal{C}=\left(\mathcal{C}^{*}(*), \mathrm{d}\right)$ of sheaves of abelian groups over $X$ together with a morphism

$$
\mathfrak{c}_{\mathcal{C}}: \mathcal{G} \rightarrow \mathcal{C}
$$

in the derived category of graded complexes of sheaves of abelian groups over $X$ such that the sheaves $\mathcal{C}^{n}(p)$ satisfy the Mayer-Vietoris principle for all $n, p$. For a $\mathcal{G}$-complex $\left(\mathcal{C}, \mathfrak{c}_{\mathcal{C}}\right)$ over $X$ we will simply write $\mathcal{C}$ as a shorthand.

Definition 3.5. A morphism of $\mathcal{G}$-complexes over $X$ is a morphism $f: \mathcal{C} \rightarrow \mathcal{C}^{\prime}$ of graded complexes of sheaves of abelian groups over $X$ such that the diagram

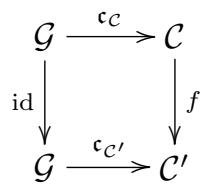

commutes in the derived category of graded complexes of sheaves of abelian groups over $X$. 
Cohomology groups of a $\mathcal{G}$-complex

Notation 3.6. For an open subset $U$ of $X$, the sections of $\mathcal{C}^{n}(p)$ over $U$ will be denoted by $\mathcal{C}^{n}(U, p)$. If $\omega \in \mathcal{C}^{n}(X, p)$, we will write $\left.\omega\right|_{U}$ for the restriction of $\omega$ to $U$; moreover, if the open set $U$ is clear from the context, we will simply write $\omega$ instead of $\left.\omega\right|_{U}$. Furthermore, if $Y$ is a closed subset of $X$ and $U=X \backslash Y$, we introduce the following notation:

$$
\begin{aligned}
H_{\mathcal{C}}^{*}(X, p) & =H^{*}(\mathcal{C}(X, p)), \\
H_{\mathcal{C}}^{*}(U, p) & =H^{*}(\mathcal{C}(U, p)), \\
H_{\mathcal{C}, Y}^{*}(X, p) & =H^{*}(\mathcal{C}(X, p), \mathcal{C}(U, p)) .
\end{aligned}
$$

Analogously, for any family $\varphi$ of supports on $X$, we define the cohomology groups with support in $\varphi$ by $H_{\mathcal{C}, \varphi}^{*}(X, p)$.

Since the sheaves $\mathcal{C}^{*}(p)$ are totally acyclic, the cohomology of global sections agrees with the hypercohomology of the complex. Hence, there are induced morphisms

$$
\begin{aligned}
H^{*}(X, \mathcal{G}(p)) & \rightarrow H_{\mathcal{C}}^{*}(X, p), \\
H^{*}(U, \mathcal{G}(p)) & \rightarrow H_{\mathcal{C}}^{*}(U, p), \\
H_{Y}^{*}(X, \mathcal{G}(p)) & \rightarrow H_{\mathcal{C}, Y}^{*}(X, p),
\end{aligned}
$$

which, by abuse of notation, will be denoted again by $\mathfrak{c}_{\mathcal{C}}$.

Lemma 3.7. Let $Y$ be a closed subset of $X$, and $U=X \backslash Y$. Then, there is a morphism of exact sequences

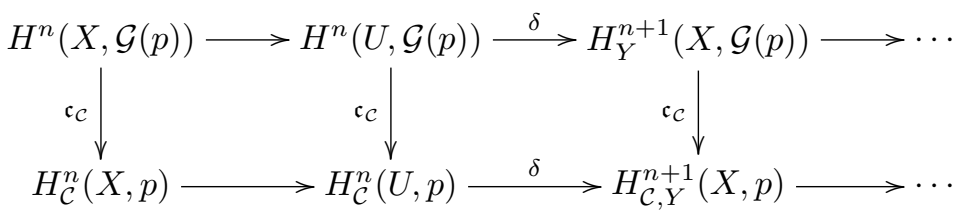

Proof. The proof follows immediately from the fact that $\mathfrak{c}_{\mathcal{C}}: \mathcal{G} \rightarrow \mathcal{C}$ is a morphism of sheaves.

\section{Characteristic classes in $\mathcal{C}$-cohomology}

Using the fact that there are well-defined characteristic classes in $\mathcal{G}$-cohomology, we can also define classes for cycles and $K_{1}$-chains in $\mathcal{C}$-cohomology.

Definition 3.8. Let $y$ be a $p$-codimensional cycle of $X$, and $Y=\operatorname{supp} y$. We define $\operatorname{cl}_{\mathcal{C}}(y) \in H_{\mathcal{C}, Y}^{2 p}(X, p)$ to be the class

$$
\operatorname{cl}_{\mathcal{C}}(y)=\mathfrak{c}_{\mathcal{C}}\left(\operatorname{cl}_{\mathcal{G}}(y)\right)
$$

Let $f \in R_{p}^{p-1}(X)$ be a $K_{1}$-chain, $y=\operatorname{div}(f), Y=\operatorname{supp} y$, and $U=X \backslash Y$. We define $\operatorname{cl}_{\mathcal{C}}(f) \in H_{\mathcal{C}}^{2 p-1}(U, p)$ to be the class

$$
\operatorname{cl}_{\mathcal{C}}(f)=\mathfrak{c}_{\mathcal{C}}\left(\operatorname{cl}_{\mathcal{G}}(f)\right) .
$$

Here $\mathrm{cl}_{\mathcal{G}}$ is given in Definition 1.50. 
If $Y=\operatorname{supp} y$ and $Y \subseteq Z$ for a closed subset $Z$ of $X$, by abuse of notation, we will also denote by $\operatorname{cl}_{\mathcal{C}}(y)$ the class in $H_{\mathcal{C}, Z}^{2 p}(X, p)$. If there is a danger of confusion, we will write explicitly $\operatorname{cl}_{\mathcal{C}}(y) \in H_{\mathcal{C}, Z}^{2 p}(X, p)$ to indicate in which cohomology group the class is considered. We will use the same convention for $\mathrm{cl}_{\mathcal{C}}(f)$. In particular, if $y=\operatorname{div}(f), Y=$ $\operatorname{supp} y, U=X \backslash Y$, and $W=\operatorname{supp} f$, we will consider the classes $\operatorname{cl}_{\mathcal{C}}(f) \in H_{\mathcal{C}}^{2 p-1}(U, p)$, and $\operatorname{cl}_{\mathcal{C}}(f) \in H_{\mathcal{C}, W \backslash Y}^{2 p-1}(U, p)$.

Observe that the cohomology $\mathcal{G}$ is only an auxiliary device to ensure the existence of classes for cycles and $K_{1}$-chains having good properties. The properties which will be needed to define arithmetic Chow groups are summarized in the following lemma. This lemma can be taken as an alternative starting point for the definition of arithmetic Chow groups. It is an immediate consequence of the properties of characteristic classes in $\mathcal{G}$-cohomology.

Lemma 3.9. Let $\mathcal{C}$ be a $\mathcal{G}$-complex over $X$. Then, we have the following statements.

(i) For any family $\varphi$ of supports on $X$, the map $\mathrm{cl}_{\mathcal{C}}$ induces a morphism of groups

$$
\mathrm{Z}_{\varphi}^{p}(X) \rightarrow H_{\mathcal{C}, \varphi}^{2 p}(X, p)
$$

which is compatible with change of support; here $\mathrm{Z}_{\varphi}^{p}(X)=R_{p, \varphi}^{p}(X)$ is the group of $p$-codimensional cycles on $X$ with support on $\varphi$.

(ii) For a $K_{1}$-chain $f \in R_{p}^{p-1}(X)$, put $y=\operatorname{div}(f), Y=\operatorname{supp} y$, and $U=X \backslash Y$. Then, the equality

$$
\operatorname{cl}_{\mathcal{C}}(\operatorname{div}(f))=\delta \operatorname{cl}_{\mathcal{C}}(f) \in H_{\mathcal{C}, Y}^{2 p}(X, p)
$$

holds, where $\delta$ is the connection morphism

$$
\delta: H_{\mathcal{C}}^{2 p-1}(U, p) \rightarrow H_{\mathcal{C}, Y}^{2 p}(X, p) .
$$

Moreover, if $g \in R_{p}^{p-1}(X)$ is another $K_{1}$-chain, $z=\operatorname{div}(g)$, and $Z=\operatorname{supp} z$, we have

$$
\operatorname{cl}_{\mathcal{C}}(f)+\operatorname{cl}_{\mathcal{C}}(g)=\operatorname{cl}_{\mathcal{C}}(f+g) \in H_{\mathcal{C}}^{2 p-1}(X \backslash(Y \cup Z), p) .
$$

(iii) If $h \in R_{p}^{p-2}(X)$ is a $K_{2}$-chain, then the class $\mathrm{cl}_{\mathcal{C}}(\mathrm{d} h)$ vanishes in the group $H_{\mathcal{C}}^{2 p-1}(X, p)$.

Lemma 3.9 has the following direct consequences.

Corollary 3.10. For any family $\varphi$ of supports on $X$, there are well-defined morphisms

$$
\begin{aligned}
\operatorname{cl}_{\mathcal{C}}: \mathrm{CH}_{\varphi}^{p}(X) \rightarrow H_{\mathcal{C}, \varphi}^{2 p}(X, p), \\
\operatorname{cl}_{\mathcal{C}}: \mathrm{CH}_{\varphi}^{p-1, p}(X) \rightarrow H_{\mathcal{C}, \varphi}^{2 p-1}(X, p) .
\end{aligned}
$$


Following [6], let us denote by $\mathcal{Z}^{p}=\mathcal{Z}^{p}(X)$ the set of all closed subsets of $X$ of codimension greater or equal to $p$ ordered by inclusion. We then write

$$
\begin{aligned}
H_{\mathcal{C}}^{n}\left(X \backslash \mathcal{Z}^{p}, p\right) & =\underset{Y \in \mathcal{Z}^{p}}{\lim _{\mathcal{C}}} H_{\mathcal{C}}^{n}(X \backslash Y, p), \\
H_{\mathcal{C}, \mathcal{Z}^{p}}^{n}(X, p) & ={\underset{Y \in \mathcal{Z}^{p}}{\lim }}_{\mathcal{C}^{n}, Y}^{n}(X, p) .
\end{aligned}
$$

Corollary 3.11. The following diagram is commutative and has exact rows:

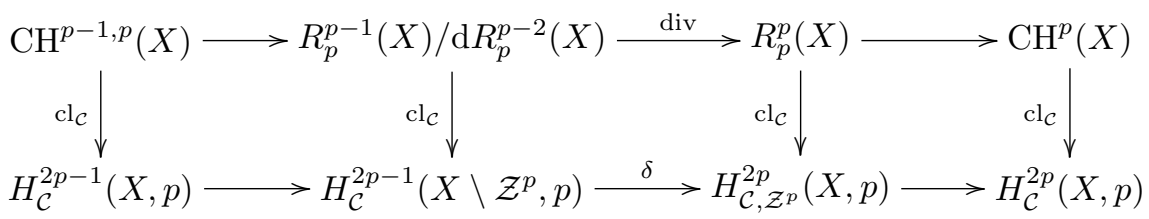

Purity

In many cases the cohomology theory associated with a $\mathcal{G}$-complex $\mathcal{C}$ will satisfy a purity axiom, e.g. if $\mathcal{C}$-cohomology and $\mathcal{G}$-cohomology agree. Later on we will see that the arithmetic Chow groups obtained in this case have better properties.

Definition 3.12. We say that the $\mathcal{G}$-complex $\mathcal{C}$ satisfies the weak purity condition, if for any closed subset $Y$ of $X$ of codimension greater or equal to $p$, we have

$$
H_{\mathcal{C}, Y}^{2 p-1}(X, p)=0
$$

Recall that for any complex $A$, we let $\widetilde{A}=A$ /Imd. In order to be able to deal with complexes which may not satisfy the weak purity condition, we introduce the following notation:

$$
\begin{aligned}
\widetilde{\mathcal{C}}^{2 p-1}(X, p)^{\text {pure }} & =\widetilde{\mathcal{C}}^{2 p-1}(X, p) / \operatorname{Im}\left(H_{\mathcal{C}, \mathcal{Z}^{p}}^{2 p-1}(X, p) \rightarrow \widetilde{\mathcal{C}}^{2 p-1}(X, p)\right) \\
H_{\mathcal{C}}^{2 p-1}(X, p)^{\text {pure }} & =H_{\mathcal{C}}^{2 p-1}(X, p) / \operatorname{Im}\left(H_{\mathcal{C}, \mathcal{Z}^{p}}^{2 p-1}(X, p) \rightarrow H_{\mathcal{C}}^{2 p-1}(X, p)\right) .
\end{aligned}
$$

Note that, if $\mathcal{C}$ satisfies the weak purity condition, then

$$
\begin{aligned}
\widetilde{\mathcal{C}}^{2 p-1}(X, p)^{\text {pure }} & =\widetilde{\mathcal{C}}^{2 p-1}(X, p), \\
H_{\mathcal{C}}^{2 p-1}(X, p)^{\text {pure }} & =H_{\mathcal{C}}^{2 p-1}(X, p) .
\end{aligned}
$$

\subsection{Definition of Green objects}

\section{Preliminaries}

Given a $\mathcal{G}$-complex $\mathcal{C}=\left(\mathcal{C}^{*}(*)\right.$,d) over $X$, we will define Green objects with values in this complex. For concrete examples we refer to [17], or $\S \S 6$ and 7 below. 
Notation 3.13. Let $Y$ be a closed subset of $X$, and $U=X \backslash Y$. We will then write

$$
\widehat{H}_{\mathcal{C}, Y}^{n}(X, p)=\widehat{H}^{n}(\mathcal{C}(X, p), \mathcal{C}(U, p)),
$$

where $\widehat{H}^{n}(\mathcal{C}(X, p), \mathcal{C}(U, p))$ are the truncated relative cohomology groups defined in $\S 2.4$. We will also write

$$
\widehat{H}_{\mathcal{C}, \mathcal{Z}^{p}}^{n}(X, p)=\underset{Y \in \mathcal{Z}^{p}}{\lim _{\mathcal{C}, Y}} \widehat{H}_{\mathcal{C}}^{n}(X, p)
$$

By writing

$$
\mathcal{C}\left(X \backslash \mathcal{Z}^{p}, p\right)=\underset{Y \in \mathcal{Z}^{p}}{\lim } \mathcal{C}(X \backslash Y, p)
$$

we have

$$
\widehat{H}_{\mathcal{C}, \mathcal{Z}^{p}}^{n}(X, p)=\widehat{H}^{n}\left(\mathcal{C}(X, p), \mathcal{C}\left(X \backslash \mathcal{Z}^{p}, p\right)\right)
$$

From the definition of the truncated relative cohomology groups we recall the morphisms

$$
\begin{aligned}
& \mathrm{cl}: \widehat{H}_{\mathcal{C}, \mathcal{Z}^{p}}^{n}(X, p) \rightarrow H_{\mathcal{C}, \mathcal{Z}^{p}}^{n}(X, p), \\
& \omega: \widehat{H}_{\mathcal{C}, \mathcal{Z}^{p}}^{n}(X, p) \rightarrow \mathrm{Z}\left(\mathcal{C}^{n}(X, p)\right), \\
& \mathrm{a}: \widetilde{\mathcal{C}}^{n-1}(X, p) \rightarrow \widehat{H}_{\mathcal{C}, \mathcal{Z}^{p}}^{n}(X, p), \\
& \mathrm{a}: H_{\mathcal{C}}^{n-1}(X, p) \rightarrow \widehat{H}_{\mathcal{C}, \mathcal{Z}^{p}}^{n}(X, p), \\
& \mathrm{b}: H_{\mathcal{C}}^{n-1}\left(X \backslash \mathcal{Z}^{p}, p\right) \rightarrow \widehat{H}_{\mathcal{C}, \mathcal{Z}^{p}}^{n}(X, p) ;
\end{aligned}
$$

analogous morphisms exist for $\widehat{H}_{\mathcal{C}, Y}^{n}(X, p)$ with $Y$ a closed subset of $X$. We leave it to the reader to write down the exact sequences of Proposition 2.58 in these cases. In particular, for $Y \in \mathcal{Z}^{p}$ we note the commutative diagram with exact rows

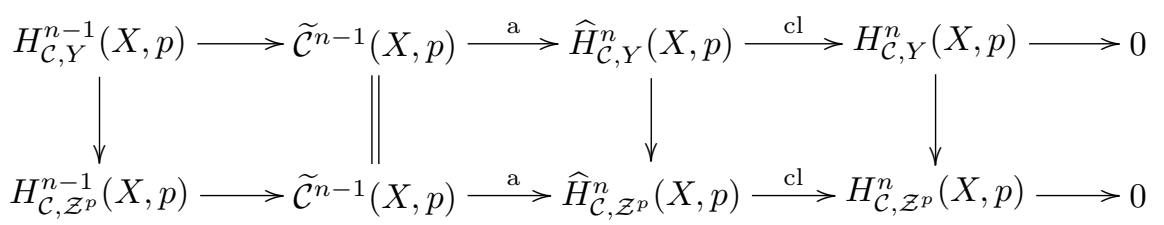

While dealing with $p$-codimensional cycles, we will be mainly interested in the groups with $n=2 p$. In this case we obtain as a direct consequence of Proposition 2.58.

Proposition 3.15. There are exact sequences

$$
\begin{aligned}
0 & \rightarrow \widetilde{\mathcal{C}}^{2 p-1}(X, p)^{\text {pure }} \stackrel{\text { a }}{\rightarrow} \widehat{H}_{\mathcal{C}, \mathcal{Z}^{p}}^{2 p}(X, p) \stackrel{\text { cl }}{\rightarrow} H_{\mathcal{C}, \mathcal{Z}^{p}}^{2 p}(X, p) \rightarrow 0, \\
0 & \rightarrow H_{\mathcal{C}}^{2 p-1}(X, p)^{\text {pure }} \stackrel{\text { a }}{\rightarrow} \widehat{H}_{\mathcal{C}, \mathcal{Z}^{p}}^{2 p}(X, p) \stackrel{\text { cl } \oplus \omega}{\rightarrow} H_{\mathcal{C}, \mathcal{Z}^{p}}^{2 p}(X, p) \oplus \mathrm{ZC}^{2 p}(X, p) \rightarrow H_{\mathcal{C}}^{2 p}(X, p) \rightarrow 0 .
\end{aligned}
$$




\section{Green objects}

Definition 3.18. Let $y$ be a $p$-codimensional cycle on $X$. A Green object for the class of $y$ (with values in $\mathcal{C}$ ) is an element $\mathfrak{g}_{y} \in \widehat{H}_{\mathcal{C}, \mathcal{Z}^{p}}^{2 p}(X, p)$ such that

$$
\operatorname{cl}\left(\mathfrak{g}_{y}\right)=\operatorname{cl}_{\mathcal{C}}(y) \in H_{\mathcal{C}, \mathcal{Z}^{p}}^{2 p}(X, p) .
$$

In other words, a Green object for the class of $y$ is a pair $\mathfrak{g}_{y}=\left(\omega_{y}, \widetilde{g}_{y}\right)$ with $\omega_{y} \in \mathrm{Z} \mathcal{C}^{2 p}(X, p)$ and $\widetilde{g}_{y} \in \widetilde{\mathcal{C}}^{2 p-1}\left(X \backslash \mathcal{Z}^{p}, p\right)$ such that $\omega_{y}=\mathrm{d} g_{y}$ and such that this pair represents the class of $y$ in $H_{\mathcal{C}, \mathcal{Z}^{p}}^{2 p}(X, p)$; note that $g_{y}$ here denotes a representative of $\widetilde{g}_{y}$ in $\mathcal{C}^{2 p-1}\left(X \backslash \mathcal{Z}^{p}, p\right)$.

Observe that the Green objects for the class of any $p$-codimensional cycle belong to the same space $\widehat{H}_{\mathcal{C}, \mathcal{Z}^{p}}^{2 p}(X, p)$. This is the reason for taking the limit over all $p$-codimensional cycles of $X$. Nevertheless, sometimes it will be necessary to have a more precise control on the group in which a Green object is defined.

Definition 3.19. Let $y$ be a $p$-codimensional cycle on $X$ with $Y=\operatorname{supp} y$. A Green object for the cycle $y$ is an element $\mathfrak{g}_{y} \in \widehat{H}_{\mathcal{C}, Y}^{2 p}(X, p)$ such that

$$
\operatorname{cl}\left(\mathfrak{g}_{y}\right)=\operatorname{cl}_{\mathcal{C}}(y) \in H_{\mathcal{C}, Y}^{2 p}(X, p) .
$$

Definition 3.20. Let $Z$ be a closed subset of $X$, and $y \in \mathrm{CH}_{Z}^{p}(X)$. A weak Green object for $y$ with support in $Z$ is an element $\mathfrak{g}_{y} \in \widehat{H}_{\mathcal{C}, Z}^{2 p}(X, p)$ such that

$$
\operatorname{cl}\left(\mathfrak{g}_{y}\right)=\operatorname{cl}_{\mathcal{C}}(y) \in H_{\mathcal{C}, Z}^{2 p}(X, p) .
$$

Remark 3.21. A Green object for the cycle $y$ represents a cohomology class with support exactly equal to the support of $y$. A Green object for the class of $y$ represents a cohomology class whose support has the same codimension as $y$, but may be bigger than the support of $y$. From Proposition 3.23 (iii) below, it will be clear that, if the $\mathcal{G}$-complex $\mathcal{C}$ satisfies the weak purity condition, there is no difference between a Green object for the class of $y$ and a Green object for the cycle $y$. As we will see, even in the case of a complex which does not satisfy the weak purity condition, the distinction between these two kinds of Green objects is a minor technical point. In contrast to this, a weak Green object for $y$ represents a cohomology class whose support may be bigger than the support of $y$, and may even have codimension smaller than the codimension of $y$. In general, a weak Green object $\mathfrak{g}_{y}$ carries less information than a Green object for $y$ and is not a Green object for the class of $y$. Weak Green objects appear naturally when handling non-proper intersections and are therefore useful in intermediate steps.

\section{Additivity}

Remark 3.22. If $\mathfrak{g}_{y}$ is a Green object for $y$, and $\mathfrak{g}_{z}$ a Green object for $z$ such that $\operatorname{supp}(y+z)=\operatorname{supp}(y) \cup \operatorname{supp}(z)$, Lemma 3.9 implies that $\mathfrak{g}_{y}+\mathfrak{g}_{z}$ is a Green object for $y+z$. On the other hand, if $\operatorname{supp}(y+z) \subsetneq \operatorname{supp}(y) \cup \operatorname{supp}(z)$, then $\mathfrak{g}_{y}+\mathfrak{g}_{z}$ determines only a Green object for the class of $y+z$. 


\section{Existence of Green objects}

Proposition 3.23. Let $\mathcal{C}$ be a $\mathcal{G}$-complex on $X$, and let $y$ be any $p$-codimensional cycle on $X$ with $Y=\operatorname{supp} y$. Then, we have the following statements.

(i) There exists a Green object $\mathfrak{g}_{y} \in \widehat{H}_{\mathcal{C}, \mathcal{Z}^{p}}^{2 p}(X, p)$ for the class of $y$.

(ii) Any Green object $\mathfrak{g}_{y}$ for the class of $y$ can be lifted to a Green object $\mathfrak{g}_{y}^{\prime} \in \widehat{H}_{\mathcal{C}, Y}^{2 p}(X, p)$ for $y$.

(iii) If the $\mathcal{G}$-complex $\mathcal{C}$ satisfies the weak purity condition, the morphism

$$
\widehat{H}_{\mathcal{C}, Y}^{2 p}(X, p) \rightarrow \widehat{H}_{\mathcal{C}, \mathcal{Z}^{p}}^{2 p}(X, p)
$$

is injective. Therefore, the Green object $\mathfrak{g}_{y}^{\prime}$ for $y$ is uniquely determined by the Green object $\mathfrak{g}_{y}$ for the class of $y$ in this case.

Proof. (i) The first statement is an immediate consequence of the surjectivity of the class map $\mathrm{cl}$ in the exact sequence (3.15).

(ii) Since the class of the cycle $y$ lies in $H_{\mathcal{C}, Y}^{2 p}(X, p)$, the second statement follows from a standard diagram chase in the commutative diagram (3.13).

(iii) If $\mathcal{C}$ satisfies the weak purity condition, the commutative diagram (3.13) gives rise to the diagram

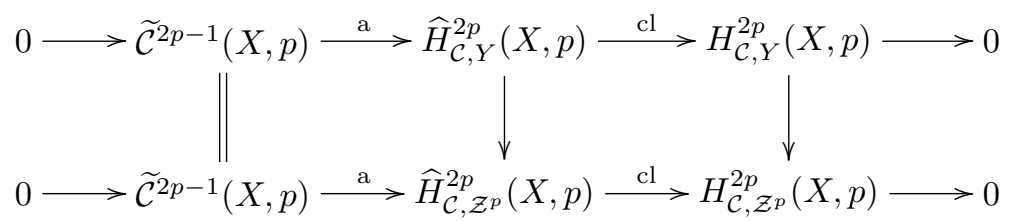

where the vertical map on the right is injective. Therefore, by the five lemma, the vertical map in the middle is also injective.

The Green object associated to a $K_{1}$-chain

Definition 3.24. For a $K_{1}$-chain $f \in R_{p}^{p-1}(X)$ with $y=\operatorname{div}(f)$, and $Y=\operatorname{supp} y$, we denote by $\mathfrak{g}(f)$ the distinguished Green object

$$
\mathfrak{g}(f)=\mathrm{b}\left(\operatorname{cl}_{\mathcal{C}}(f)\right) \in \widehat{H}_{\mathcal{C}, Y}^{2 p}(X, p) .
$$

If we need to specify the $\mathcal{G}$-complex $\mathcal{C}$, we will write $\mathfrak{g}_{\mathcal{C}}(f)$ instead of $\mathfrak{g}(f)$.

Remark 3.25. By Lemma $3.9(\mathrm{ii}), \mathfrak{g}(f)$ is a well-defined Green object for $y=\operatorname{div} f$. By Lemma 3.9 (iii), we have $\mathfrak{g}(\mathrm{d} h)=0$ for any $K_{2}$-chain $h$. This means in particular that the distinguished Green object $\mathfrak{g}(f)$ depends only on the class of $f$ in $\mathrm{CH}^{p-1, p}(X \backslash Y)$. In other words, for any closed subset $Z$ of $X$ and $U=X \backslash Z$, there is a well-defined morphism

$$
\mathrm{CH}^{p-1, p}(U) \rightarrow \widehat{H}_{\mathcal{C}, Z}^{2 p}(X, p)
$$

which we also denote by $\mathfrak{g}$. 


\subsection{The *-product of Green objects}

In this section we will define a pairing of Green objects, the *-product, following the strategy of Proposition 2.68. We will define the $*$-product for any pairing of $\mathcal{G}$ complexes which is compatible with the product given in $\mathcal{G}$-cohomology. This will ensure the compatibility with the intersection product of cycles.

Pairing of $\mathcal{G}$-complexes

Definition 3.26. Let $\mathcal{C}, \mathcal{C}^{\prime}$ and $\mathcal{C}^{\prime \prime}$ be $\mathcal{G}$-complexes over $X$ with the same auxiliary cohomology $\mathcal{G}$. A $\mathcal{G}$-pairing is a pairing of graded complexes of sheaves of abelian groups

$$
\mathcal{C} \otimes \mathcal{C}^{\prime} \stackrel{\bullet}{\rightarrow} \mathcal{C}^{\prime \prime}
$$

such that, in the derived category of graded complexes of sheaves of abelian groups over $X$, there is a commutative diagram

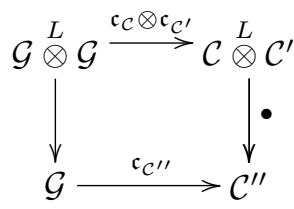

where the vertical arrow on the left is the product in $\mathcal{G}$-cohomology (see $[\mathbf{3 4}, 1.1]$ ).

The *-product of Green objects

Definition 3.27. Let $\mathcal{C}$ be a $\mathcal{G}$-complex over $X$. Let $Y, Z$ be closed subsets of $X$, $U=X \backslash Y, V=X \backslash Z$, respectively, and let

$$
j: \mathcal{C}^{n}(U, p) \oplus \mathcal{C}^{n}(V, p) \rightarrow \mathcal{C}^{n}(U \cap V, p)
$$

be the map given by $j(\omega, \eta)=-\omega+\eta$; we then put

$$
\mathcal{C}(X ; Y, Z, p)=s(-j) \text {. }
$$

Observe that this complex is, up to the sign of the morphism, the Čech complex associated to the sheaf $\mathcal{C}$ and the open covering $\{U, V\}$.

The kernel-simple quasi-isomorphism (see Definition 2.37) associated to the short exact sequence

$$
0 \rightarrow \mathcal{C}^{n}(U \cup V, p) \stackrel{i}{\rightarrow} \mathcal{C}^{n}(U, p) \oplus \mathcal{C}^{n}(V, p) \stackrel{j}{\rightarrow} \mathcal{C}^{n}(U \cap V, p) \rightarrow 0
$$

with $i(\eta)=(\eta, \eta)$, is the induced morphism

$$
\iota: \mathcal{C}(U \cup V, p) \rightarrow \mathcal{C}(X ; Y, Z, p) .
$$

Throughout this section we will assume that there are given three $\mathcal{G}$-complexes $\mathcal{C}, \mathcal{C}^{\prime}$, $\mathcal{C}^{\prime \prime}$ over $X$, and that $\bullet$ is a $\mathcal{G}$-pairing between $\mathcal{C}$ and $\mathcal{C}^{\prime}$ to $\mathcal{C}^{\prime \prime}$. Furthermore, let $Y, Z$ be closed subsets of $X$, and $U=X \backslash Y, V=X \backslash Z$, respectively. Since $\bullet$ is a pairing of 
sheaves, and therefore compatible with restrictions, it induces for all $p, q$ a morphism of commutative diagrams

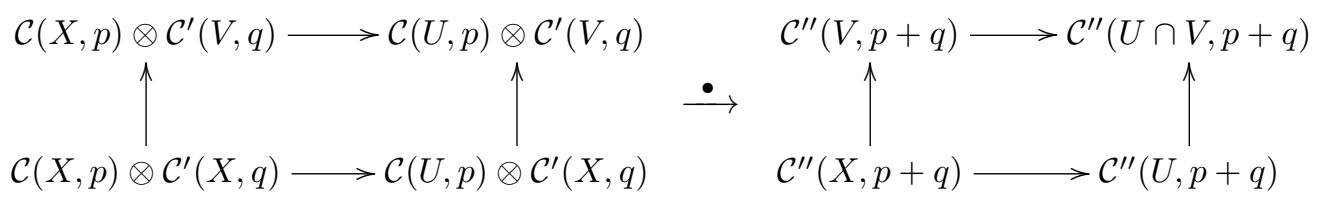

Theorem 3.31. There exist well-defined pairings and a commutative diagram

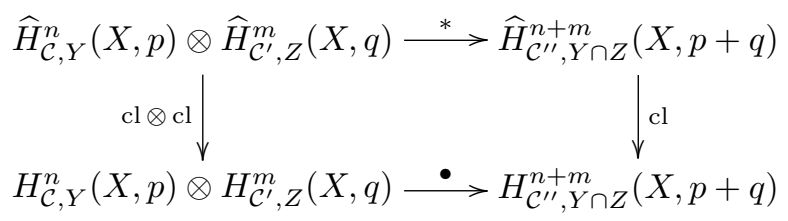

Proof. By Proposition 2.47 and Proposition 2.66, we have with $r=p+q$, well-defined pairings and a commutative diagram

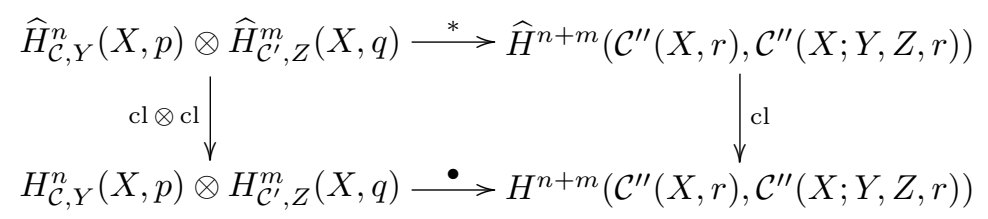

Using the kernel-simple quasi-isomorphism (3.28) and Corollary 2.63, we obtain the isomorphisms

$$
\begin{aligned}
& H_{\mathcal{C}^{\prime \prime}, Y \cap Z}^{n+m}(X, r) \stackrel{\cong}{\rightrightarrows} H^{n+m}\left(\mathcal{C}^{\prime \prime}(X, r), \mathcal{C}^{\prime \prime}(X ; Y, Z, r)\right), \\
& \widehat{H}_{\mathcal{C}^{\prime \prime}, Y \cap Z}^{n+m}(X, r) \stackrel{\cong}{\rightarrow} \widehat{H}^{n+m}\left(\mathcal{C}^{\prime \prime}(X, r), \mathcal{C}^{\prime \prime}(X ; Y, Z, r)\right) .
\end{aligned}
$$

This completes the proof of the claim.

Remark 3.35. Let $(\omega, \widetilde{g}) \in \widehat{H}_{\mathcal{C}, Y}^{n}(X, p)$, and $\left(\omega^{\prime}, \widetilde{g}^{\prime}\right) \in \widehat{H}_{\mathcal{C}^{\prime}, Z}^{m}(X, q)$. A representative of $(\omega, \widetilde{g}) *\left(\omega^{\prime}, \widetilde{g}^{\prime}\right)$ in the group $\hat{H}^{n+m}\left(\mathcal{C}^{\prime \prime}(X, r), \mathcal{C}^{\prime \prime}(X ; Y, Z, r)\right)$ is then given by formula (2.64). Observe that, in order to obtain an explicit representative for this class in the group

$$
\widehat{H}_{\mathcal{C}^{\prime \prime}, Y \cap Z}^{n+m}(X, p+q)=\widehat{H}^{n+m}\left(\mathcal{C}^{\prime \prime}(X, p+q), \mathcal{C}^{\prime \prime}(X \backslash(Y \cap Z), p+q)\right),
$$

we have to use an explicit quasi-inverse of the kernel-simple quasi-isomorphism. This will be done in the examples (see, for example, $\S 6.1$ ). 
The *-product and the intersection product

The above pairing induced by • in cohomology with support is compatible with the product defined in $\mathcal{G}$-cohomology.

Theorem 3.36. There is a commutative diagram

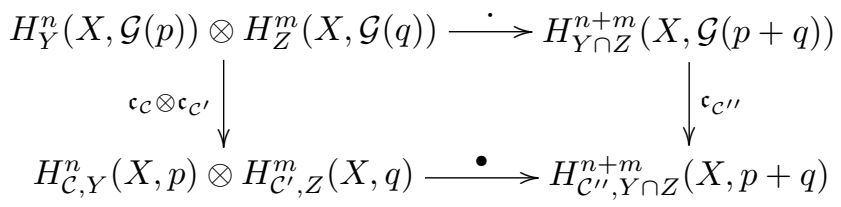

Proof. The proof follows from standard arguments in sheaf theory (see $[\mathbf{3 8}, 6.2],[\mathbf{4 7}$, II.10], [16, 2.5] and Example 2.52).

Theorem 3.37. Let $y \in \mathrm{CH}_{Y}^{p}(X), z \in \mathrm{CH}_{Z}^{q}(X)$ with closed subsets $Y, Z$ of $X$, respectively. Let $\mathfrak{g}_{y} \in \widehat{H}_{\mathcal{C}, Y}^{2 p}(X, p), \mathfrak{g}_{z} \in \widehat{H}_{\mathcal{C}^{\prime}, Z}^{2 q}(X, q)$ be weak Green objects for $y, z$, respectively, and let $w=y \cdot z \in \mathrm{CH}_{Y \cap Z}^{p+q}(X)$. Then, the *-product $\mathfrak{g}_{y} * \mathfrak{g}_{z}$ is a weak Green object for $w$. Moreover, if $\mathfrak{g}_{y}=\left(\omega_{y}, \widetilde{g}_{y}\right)$, and $\mathfrak{g}_{z}=\left(\omega_{z}, \widetilde{g}_{z}\right)$, then $\mathfrak{g}_{y} * \mathfrak{g}_{z}$ is represented in the group $\widehat{H}^{2 p+2 q}\left(\mathcal{C}^{\prime \prime}(X, p+q), \mathcal{C}^{\prime \prime}(X ; Y, Z, p+q)\right)$ by the element

$$
\left(\omega_{y} \bullet \omega_{z},\left(\left(g_{y} \bullet \omega_{z}, \omega_{y} \bullet g_{z}\right),-g_{y} \bullet g_{z}\right)^{\sim}\right) .
$$

Proof. We have to show that $\operatorname{cl}\left(\mathfrak{g}_{y} * \mathfrak{g}_{z}\right)=\operatorname{cl}_{\mathcal{C}^{\prime \prime}}(w) \in H_{\mathcal{C}^{\prime \prime}, Y \cap Z}^{2 p+2 q}(X, p+q)$. But by Theorem 3.31, we have

$$
\operatorname{cl}\left(\mathfrak{g}_{y} * \mathfrak{g}_{z}\right)=\operatorname{cl}\left(\mathfrak{g}_{y}\right) \bullet \operatorname{cl}\left(\mathfrak{g}_{z}\right)=\operatorname{cl}_{\mathcal{C}}(y) \bullet \operatorname{cl}_{\mathcal{C}^{\prime}}(z)=\mathfrak{c}_{\mathcal{C}}\left(\operatorname{cl}_{\mathcal{G}}(y)\right) \bullet \mathfrak{c}_{\mathcal{C}^{\prime}}\left(\operatorname{cl}_{\mathcal{G}}(z)\right) .
$$

Now, by Theorem 3.36, $\mathfrak{c}_{\mathcal{C}}\left(\operatorname{cl}_{\mathcal{G}}(y)\right) \bullet \mathfrak{c}_{\mathcal{C}^{\prime}}\left(\operatorname{cl}_{\mathcal{G}}(z)\right)=\mathfrak{c}_{\mathcal{C}^{\prime \prime}}\left(\operatorname{cl}_{\mathcal{G}}(y) \cdot \operatorname{cl}_{\mathcal{G}}(z)\right)$. Finally, by Proposition 1.54, $\operatorname{cl}_{\mathcal{G}}(y) \cdot \operatorname{cl}_{\mathcal{G}}(z)=\operatorname{cl}_{\mathcal{G}}(w)$. The claimed formula (3.37) follows from (2.64) taking into account the degrees of $\omega_{y}$ and $g_{y}$.

Theorem 3.39. Let $y$ be a $p$-codimensional, respectively $z$ a $q$-codimensional, cycle of $X$, and $Y=\operatorname{supp} y$, respectively $Z=\operatorname{supp} z$. Let $\mathfrak{g}_{y} \in \widehat{H}_{\mathcal{C}, \mathcal{Z}^{p}}^{2 p}(X, p)$, respectively $\mathfrak{g}_{z} \in \widehat{H}_{\mathcal{C}^{\prime}, \mathcal{Z}^{q}}^{2 q}(X, q)$ be Green objects for the class of $y$, respectively $z$. Let $\mathfrak{g}_{y}^{\prime} \in \widehat{H}_{\mathcal{C}, Y}^{2 p}(X, p)$, respectively $\mathfrak{g}_{z}^{\prime} \in \widehat{H}_{\mathcal{C}^{\prime}, Z}^{2 q}(X, q)$ be representatives of $\mathfrak{g}_{y}$, respectively $\mathfrak{g}_{z}$. If $y$ and $z$ intersect properly, we have the following statements.

(i) The product $\mathfrak{g}_{y}^{\prime} * \mathfrak{g}_{z}^{\prime}$ is a Green object for the cycle $y \cdot z$.

(ii) The image of $\mathfrak{g}_{y}^{\prime} * \mathfrak{g}_{z}^{\prime}$ in $\widehat{H}_{\mathcal{C}^{\prime \prime}, \mathcal{Z}^{p+q}}^{2 p+2 q}(X, p+q)$ does not depend on the choice of the representatives $\mathfrak{g}_{y}^{\prime}$ and $\mathfrak{g}_{z}^{\prime}$.

Proof. The first statement is a direct consequence of Theorem 3.37. Observe that, if the complexes $\mathcal{C}$ and $\mathcal{C}^{\prime}$ satisfy the weak purity condition, the second statement is automatically fulfilled. 
To prove the second statement in the case in which the weak purity condition is not satisfied, we let $\mathfrak{g}_{y}^{\prime \prime}$ be another choice for a lift of $\mathfrak{g}_{y}$. We then consider the commutative diagram (3.13), i.e.

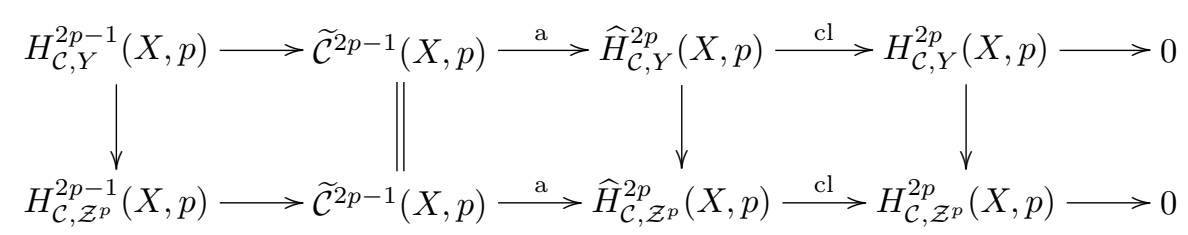

Since we are not assuming weak purity, the vertical map on the right of this diagram need not be injective. But, by the definition of a Green object for a cycle, we know that the images of $\mathfrak{g}_{y}^{\prime}$ and $\mathfrak{g}_{y}^{\prime \prime}$ by the class map agree in $H_{\mathcal{C}, Y}^{2 p}(X, p)$. Therefore, we have

$$
\mathfrak{g}_{y}^{\prime}-\mathfrak{g}_{y}^{\prime \prime}=\mathrm{a}(\widetilde{x})
$$

for some $x \in \mathcal{C}^{2 p-1}(X, p)$. Moreover, since $\mathfrak{g}_{y}^{\prime}$ and $\mathfrak{g}_{y}^{\prime \prime}$ have the same image in $\widehat{H}_{\mathcal{C}, \mathcal{Z}^{p}}^{2 p}(X, p)$, we have a $(\widetilde{x})=0$ in the group $\widehat{H}_{\mathcal{C}, \mathcal{Z}^{p}}^{2 p}(X, p)$ which implies $\omega(\mathrm{a}(\widetilde{x}))=0$, and hence shows $\mathrm{d} x=0$. Therefore, $\widetilde{x} \in H_{\mathcal{C}, \mathcal{Z}^{p}}^{2 p-1}(X, p) \subseteq \widetilde{\mathcal{C}}^{2 p-1}(X, p)$ which implies that $\widetilde{x}$ arises as the image of a class in $H_{\mathcal{C}, W}^{2 p-1}(X, p)$ for a suitable closed subset $W$ of $X$ of codimension greater or equal to $p$. Writing $\mathfrak{g}_{z}^{\prime}=\left(\omega_{z}, \widetilde{g}_{z}^{\prime}\right)$, we obtain by Proposition 2.67 (iii), the equality

$$
\mathrm{a}(\widetilde{x}) * \mathfrak{g}_{z}^{\prime}=\mathrm{a}\left(\widetilde{x \bullet \omega_{z}}\right)
$$

in the group $\widehat{H}_{\mathcal{C}^{\prime \prime}, W \cap Z}^{2 p+2 q}(X, p+q)$. By the moving lemma for cycles, there exists a cycle $z^{\prime}$ linearly equivalent to $z$ which intersects $W$ properly. Since the class of $z^{\prime}$ in $H_{\mathcal{C}^{\prime}}^{2 q}(X, q)$ can also be represented by $\omega_{z}$, the class

$$
\widetilde{x \bullet \omega_{z}} \in \widetilde{\mathcal{C}}^{\prime \prime 2 p+2 q-1}(X, p+q)
$$

arises as the image of a class with support in the closed subset $W \cap \operatorname{supp} z^{\prime}$ which has codimension greater or equal to $p+q$; this shows that this class also arises as the image of a class in $H_{\mathcal{C}^{\prime \prime}}^{2 p+2 q-1} \mathcal{Z}^{p+q}(X, p+q)$. By exactness, the element a $\left(\widetilde{x \bullet \omega_{z}}\right)$ therefore vanishes in $\widehat{H}_{\mathcal{C}^{\prime \prime}, \mathcal{Z}^{p+q}}^{2 p+2 q}(X, p+q)$. This proves the claim.

Definition 3.40. Let $y$ be a $p$-codimensional cycle on $X, \mathfrak{g}_{y} \in \widehat{H}_{\mathcal{C}, \mathcal{Z}^{p}}^{2 p}(X, p)$ a Green object for the class of $y$; let $z$ be a $q$-codimensional cycle on $X, \mathfrak{g}_{z} \in \widehat{H}_{\mathcal{C}^{\prime}, \mathcal{Z}^{q}}^{2 q}(X, q)$ a Green object for the class of $z$. Then, the *-product of $\mathfrak{g}_{y}$ with $\mathfrak{g}_{z}$, denoted by $\mathfrak{g}_{y} * \mathfrak{g}_{z}$, is defined as the image of $\mathfrak{g}_{y}^{\prime} * \mathfrak{g}_{z}^{\prime}$ in the group $\widehat{H}_{\mathcal{C}^{\prime \prime}, \mathcal{Z}^{p+q}}^{2 p+2 q}(X, p+q)$, where $\mathfrak{g}_{y}^{\prime}$, respectively $\mathfrak{g}_{z}^{\prime}$, is any lift of $\mathfrak{g}_{y}$, respectively $\mathfrak{g}_{z}$.

Note that, in the above definition, the image of $\mathfrak{g}_{y} * \mathfrak{g}_{z} \in \widehat{H}_{\mathcal{C}^{\prime \prime}, \mathcal{Z}^{p+q}}^{2 p+2 q}(X, p+q)$ is a welldefined Green object for the class of $y \cdot z$ by Theorem 3.39 . 


\subsection{Associativity and commutativity}

\section{Associativity}

Let $\mathcal{C}_{1}, \mathcal{C}_{2}, \mathcal{C}_{3}, \mathcal{C}_{12}, \mathcal{C}_{23}, \mathcal{C}_{123}$ be $\mathcal{G}$-complexes and assume that there are $\mathcal{G}$-pairings

$$
\begin{aligned}
\mathcal{C}_{1} \otimes \mathcal{C}_{2} & \rightarrow \mathcal{C}_{12}, \\
\mathcal{C}_{2} \otimes \mathcal{C}_{3} & \rightarrow \mathcal{C}_{23}, \\
\mathcal{C}_{12} \otimes \mathcal{C}_{3} & \rightarrow \mathcal{C}_{123}, \\
\mathcal{C}_{1} \otimes \mathcal{C}_{23} & \rightarrow \mathcal{C}_{123}
\end{aligned}
$$

which we will denote collectively by $\bullet$. Recall that the product $\bullet$ is called associative up to homotopy, if there exist sheaf morphisms

$$
h_{a}: \mathcal{C}_{1}^{n}(\cdot, p) \otimes \mathcal{C}_{2}^{m}(\cdot, q) \otimes \mathcal{C}_{3}^{l}(\cdot, r) \rightarrow \mathcal{C}_{123}^{n+m+l-1}(\cdot, p+q+r)
$$

satisfying

$$
(\alpha \bullet \beta) \bullet \gamma-\alpha \bullet(\beta \bullet \gamma)=\mathrm{d} h_{a}(\alpha \otimes \beta \otimes \gamma)+h_{a} \mathrm{~d}(\alpha \otimes \beta \otimes \gamma)
$$

In order to have an associative product in the arithmetic Chow groups, we will need a slightly stronger condition than associativity up to homotopy.

Definition 3.41. The product $\bullet$ is called pseudo-associative, if it is associative up to homotopy and the equality

$$
h_{a}(\alpha \otimes \beta \otimes \gamma)=0
$$

holds for all $\alpha \in \mathrm{ZC}_{1}^{2 p}(X, p), \beta \in \mathrm{ZC}_{2}^{2 q}(X, q), \gamma \in \mathrm{ZC}_{3}^{2 r}(X, r)$.

Theorem 3.42. Assume that the product $\bullet$ is associative up to homotopy. Furthermore, let $Y, Z, W$ be closed subsets of $X$, and $\mathfrak{g}_{1} \in \widehat{H}_{\mathcal{C}_{1}, Y}^{2 p}(X, p), \mathfrak{g}_{2} \in \widehat{H}_{\mathcal{C}_{2}, Z}^{2 q}(X, q)$, $\mathfrak{g}_{3} \in \widehat{H}_{\mathcal{C}_{3}, W}^{2 r}(X, r)$ with $\mathfrak{g}_{j}=\left(\omega_{j}, \widetilde{g}_{j}\right)$ for $j=1,2,3$. Then, we have the following statements.

(i) In the group $\widehat{H}_{\mathcal{C}_{123}, Y \cap Z \cap W}^{2 p+2 q+2 r}(X, p+q+r)$, there is an equality

$$
\left(\mathfrak{g}_{1} * \mathfrak{g}_{2}\right) * \mathfrak{g}_{3}-\mathfrak{g}_{1} *\left(\mathfrak{g}_{2} * \mathfrak{g}_{3}\right)=\mathrm{a}\left(h_{a}\left(\omega_{1} \otimes \omega_{2} \otimes \omega_{3}\right)\right) .
$$

(ii) For $\mathfrak{g}_{1}$, or $\mathfrak{g}_{2}$, or $\mathfrak{g}_{3} \in \operatorname{Ker}(\omega)$, the equality

$$
\left(\mathfrak{g}_{1} * \mathfrak{g}_{2}\right) * \mathfrak{g}_{3}=\mathfrak{g}_{1} *\left(\mathfrak{g}_{2} * \mathfrak{g}_{3}\right)
$$

holds.

(iii) Moreover, if the product $\bullet$ is pseudo-associative, again the equality

$$
\left(\mathfrak{g}_{1} * \mathfrak{g}_{2}\right) * \mathfrak{g}_{3}=\mathfrak{g}_{1} *\left(\mathfrak{g}_{2} * \mathfrak{g}_{3}\right)
$$

holds. 
Proof. The statements (ii) and (iii) follow immediately from (i). To prove (i), let us write $t=p+q+r$, and let

$$
\begin{aligned}
& f_{1}: \mathcal{C}_{1}(X, p) \rightarrow \mathcal{C}_{1}(X \backslash Y, p), \\
& f_{2}: \mathcal{C}_{2}(X, p) \rightarrow \mathcal{C}_{2}(X \backslash Z, p), \\
& f_{3}: \mathcal{C}_{3}(X, p) \rightarrow \mathcal{C}_{3}(X \backslash W, p)
\end{aligned}
$$

be the restriction morphisms. For simplicity, let us denote $\mathcal{C}_{123}$ by $\mathcal{C}$. By means of the identification (2.24) we now observe that the product $\bullet$ induces a morphism of 2 -iterated complexes between $f_{1} \otimes f_{2} \otimes f_{3}$ and the 2-iterated complex

$$
\begin{aligned}
\xi=(\mathcal{C}(X, t) & \stackrel{\delta_{1}}{\longrightarrow} \mathcal{C}(X \backslash Y, t) \oplus \mathcal{C}(X \backslash Z, t) \oplus \mathcal{C}(X \backslash W, t) \\
& \stackrel{\delta_{2}}{\longrightarrow} \mathcal{C}(X \backslash(Y \cup Z), t) \oplus \mathcal{C}(X \backslash(Y \cup W), t) \oplus \mathcal{C}(X \backslash(Z \cup W), t) \\
& \left.\stackrel{\delta_{3}}{\longrightarrow} \mathcal{C}(X \backslash(Y \cup Z \cup W), t)\right) ;
\end{aligned}
$$

here

$$
\begin{aligned}
\delta_{1}(a) & =(a, a, a), \\
\delta_{2}(a, b, c) & =(b-a, c-a, c-b), \\
\delta_{3}(a, b, c) & =a-b+c .
\end{aligned}
$$

Let us denote by $\mathcal{C}(X ; Y, Z, W, t)$ the simple complex of the 2-iterated complex

$$
\begin{aligned}
\mathcal{C}(X \backslash Y, t) \oplus & \mathcal{C}(X \backslash Z, t) \oplus \mathcal{C}(X \backslash W, t) \\
& \stackrel{-\delta_{2}}{\longrightarrow} \mathcal{C}(X \backslash(Y \cup Z), t) \oplus \mathcal{C}(X \backslash(Y \cup W), t) \oplus \mathcal{C}(X \backslash(Z \cup W), t) \\
& \stackrel{-\delta_{3}}{\longrightarrow} \mathcal{C}(X \backslash(Y \cup Z \cup W), t) .
\end{aligned}
$$

Except for the signs of the morphisms this is the Čech complex associated to the open covering $\{X \backslash Y, X \backslash Z, X \backslash W\}$. As in Proposition 2.16, there is a natural isomorphism

$$
s(\xi) \rightarrow s\left(\mathcal{C}(X, t) \stackrel{\delta_{1}}{\longrightarrow} \mathcal{C}(X ; Y, Z, W, t)\right)
$$

which is given by the identity on the level of elements.

Let us consider the commutative diagram

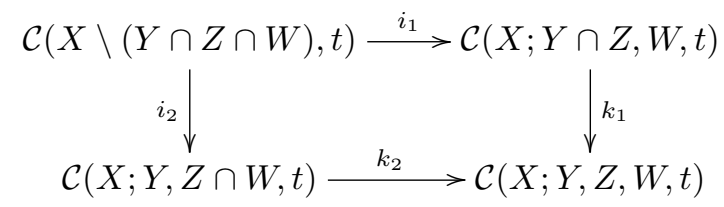

where

$$
\begin{aligned}
i_{1}(a) & =((a, a), 0), \\
i_{2}(a) & =((a, a), 0), \\
k_{1}((a, b), c) & =((a, a, b),(0, c, c), 0), \\
k_{2}((a, b), c) & =((a, b, b),(c, c, 0), 0) .
\end{aligned}
$$


The Mayer-Vietoris principle of the sheaf $\mathcal{C}$ implies that all the arrows in the above diagram are quasi-isomorphisms.

Recalling $\mathcal{C}(X ; Y, Z, t)$ from Definition 3.27, we introduce the notation

$$
\begin{aligned}
\widehat{H}_{\mathcal{C}, Y, Z}^{2 t}(X, t) & =\widehat{H}^{2 t}(\mathcal{C}(X, t), \mathcal{C}(X ; Y, Z, t)), \\
\widehat{H}_{\mathcal{C}, Y, Z, W}^{2 t}(X, t) & =\widehat{H}^{2 t}(\mathcal{C}(X, t), \mathcal{C}(X ; Y, Z, W, t)),
\end{aligned}
$$

and similar notation for the other closed subsets under consideration. We then obtain from the commutative diagram (3.45) and Corollary 2.63 the commutative diagram

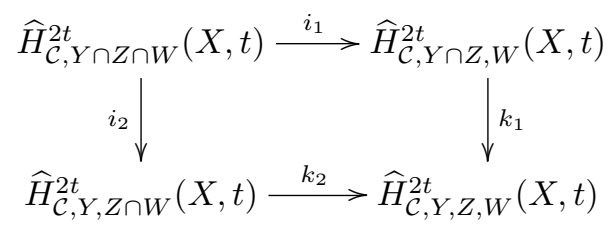

where all the arrows are isomorphisms. Therefore, in order to prove equation (3.42), it is enough to compare explicit representatives of both elements in the group $\widehat{H}_{\mathcal{C}, Y, Z, W}^{2 t}(X, t)$.

To obtain these representatives, we consider the commutative diagram

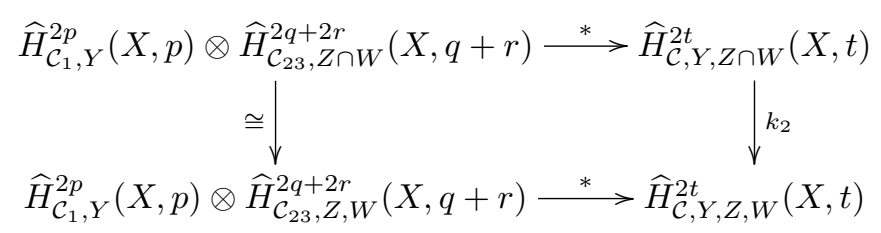

where the bottom arrow is induced from the morphism $\beta_{1}$ given in Proposition 2.26. We thus obtain

$$
(a, b) *(c,(d, e), f)=(a \bullet c,(b \bullet c, a \bullet d, a \bullet e),(-b \bullet d,-b \bullet e, a \bullet f), b \bullet f) .
$$

Analogously, there is another commutative diagram

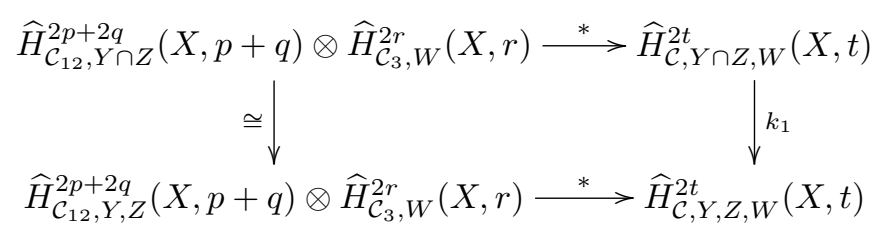

where the bottom arrow is induced from the morphism $\beta_{2}$ given in Proposition 2.26. We obtain

$$
(a,(b, c), d) *(e, f)=(a \bullet e,(b \bullet e, c \bullet e, a \bullet f),(d \bullet e,-b \bullet f,-c \bullet f), d \bullet f) .
$$

Summarizing, we find the following identities in the group $\widehat{H}_{\mathcal{C}, Y, Z, W}^{2 t}(X, t)$

$$
\begin{aligned}
\left(\mathfrak{g}_{1} * \mathfrak{g}_{2}\right) * \mathfrak{g}_{3}= & \left(\left(\omega_{1} \bullet \omega_{2}\right) \bullet \omega_{3},\left(\left(\left(g_{1} \bullet \omega_{2}\right) \bullet \omega_{3},\left(\omega_{1} \bullet g_{2}\right) \bullet \omega_{3},\left(\omega_{1} \bullet \omega_{2}\right) \bullet g_{3}\right),\right.\right. \\
& \left.\left.\left(-\left(g_{1} \bullet g_{2}\right) \bullet \omega_{3},-\left(g_{1} \bullet \omega_{2}\right) \bullet g_{3},-\left(\omega_{1} \bullet g_{2}\right) \bullet g_{3}\right),-\left(g_{1} \bullet g_{2}\right) \bullet g_{3}\right)^{\sim}\right),
\end{aligned}
$$


and

$$
\begin{aligned}
\mathfrak{g}_{1} *\left(\mathfrak{g}_{2} * \mathfrak{g}_{3}\right)= & \left(\omega_{1} \bullet\left(\omega_{2} \bullet \omega_{3}\right),\left(\left(g_{1} \bullet\left(\omega_{2} \bullet \omega_{3}\right), \omega_{1} \bullet\left(g_{2} \bullet \omega_{3}\right), \omega_{1} \bullet\left(\omega_{2} \bullet g_{3}\right)\right),\right.\right. \\
& \left.\left.\left(-g_{1} \bullet\left(g_{2} \bullet \omega_{3}\right),-g_{1} \bullet\left(\omega_{2} \bullet g_{3}\right),-\omega_{1} \bullet\left(g_{2} \bullet g_{3}\right)\right),-g_{1} \bullet\left(g_{2} \bullet g_{3}\right)\right)^{\sim}\right) .
\end{aligned}
$$

With the element $y \in \mathcal{C}^{2 t-2}(X ; Y, Z, W, t)$ given by

$$
\begin{aligned}
y=\left(\left(h_{a}\left(g_{1} \otimes \omega_{2} \otimes \omega_{3}\right), h_{a}\left(\omega_{1} \otimes g_{2} \otimes \omega_{3}\right), h_{a}\left(\omega_{1} \otimes \omega_{2} \otimes g_{3}\right)\right),\right. \\
\left.\left(h_{a}\left(g_{1} \otimes g_{2} \otimes \omega_{3}\right), h_{a}\left(g_{1} \otimes \omega_{2} \otimes g_{3}\right), h_{a}\left(\omega_{1} \otimes g_{2} \otimes g_{3}\right)\right),-h_{a}\left(g_{1} \otimes g_{2} \otimes g_{3}\right)\right),
\end{aligned}
$$

we derive the identity

$$
\left(\mathfrak{g}_{1} * \mathfrak{g}_{2}\right) * \mathfrak{g}_{3}-\mathfrak{g}_{1} *\left(\mathfrak{g}_{2} * \mathfrak{g}_{3}\right)-(0, \mathrm{~d} y)=\mathrm{a}\left(h_{a}\left(\omega_{1} \otimes \omega_{2} \otimes \omega_{3}\right)\right),
$$

which proves (i).

\section{Commutativity}

Let $\mathcal{C}_{1}$ and $\mathcal{C}$ be $\mathcal{G}$-complexes and let

$$
\bullet: \mathcal{C}_{1} \otimes \mathcal{C}_{1} \rightarrow \mathcal{C}
$$

be a $\mathcal{G}$-pairing. Recall that the pairing $\bullet$ is commutative up to homotopy, if there exist sheaf morphisms

$$
h_{c}: \mathcal{C}_{1}^{n}(\cdot, p) \otimes \mathcal{C}_{1}^{m}(\cdot, q) \rightarrow \mathcal{C}^{n+m-1}(\cdot, p+q)
$$

satisfying

$$
\alpha \bullet \beta-(-1)^{n m} \beta \bullet \alpha=\mathrm{d} h_{c}(\alpha \otimes \beta)+h_{c} \mathrm{~d}(\beta \otimes \alpha) .
$$

As in the case of the associativity, in order to have a commutative product on the level of arithmetic groups, we need a slightly stronger condition than commutativity up to homotopy.

Definition 3.48. The product $\bullet$ is called pseudo-commutative, if it is commutative up to homotopy and if the equality

$$
h_{c}(\alpha \otimes \beta)=0
$$

holds for all $\alpha \in \mathrm{ZC}_{1}^{2 p}(X, p)$ and $\beta \in \mathrm{ZC}_{1}^{2 q}(X, q)$.

Theorem 3.49. Let $Y$ and $Z$ be closed subsets of $X$, and $\mathfrak{g}_{1} \in \widehat{H}_{\mathcal{C}_{1}, Y}^{2 p}(X, p), \mathfrak{g}_{2} \in$ $\widehat{H}_{\mathcal{C}_{1}, Z}^{2 q}(X, q)$ with $\mathfrak{g}_{j}=\left(\omega_{j}, \widetilde{g}_{j}\right)$ for $j=1,2$. Then, we have the following statements.

(i) In the group $\widehat{H}_{\mathcal{C}, Y \cap Z}^{2 p+2 q}(X, p+q)$, there is an equality

$$
\mathfrak{g}_{1} * \mathfrak{g}_{2}-\mathfrak{g}_{2} * \mathfrak{g}_{1}=\mathrm{a}\left(h_{c}\left(\omega_{1} \otimes \omega_{2}\right)\right) .
$$

(ii) For $\mathfrak{g}_{1}$ or $\mathfrak{g}_{2} \in \operatorname{Ker}(\omega)$, the equality

$$
\mathfrak{g}_{1} * \mathfrak{g}_{2}=\mathfrak{g}_{2} * \mathfrak{g}_{1}
$$

holds. 
(iii) Moreover, if the product $\bullet$ is pseudo-commutative, again the equality

$$
\mathfrak{g}_{1} * \mathfrak{g}_{2}=\mathfrak{g}_{2} * \mathfrak{g}_{1}
$$

holds.

Proof. The statements (ii) and (iii) follow immediately from (i). To prove (i), let us write $s=p+q$. We recall that $\mathfrak{g}_{1} * \mathfrak{g}_{2}$ is defined as an element of

$$
\widehat{H}_{\mathcal{C}, Y, Z}^{2 s}(X, s)=\widehat{H}^{2 s}(\mathcal{C}(X, s), \mathcal{C}(X ; Y, Z, s))
$$

with $\mathcal{C}(X ; Y, Z, s)$ given in Definition 3.27. By means of the inverse of the isomorphism

$$
\widehat{H}_{\mathcal{C}, Y \cap Z}^{2 s}(X, s) \stackrel{\cong}{\longrightarrow} \widehat{H}_{\mathcal{C}, Y, Z}^{2 s}(X, s),
$$

which is induced by the kernel-simple quasi-isomorphism, the element $\mathfrak{g}_{1} * \mathfrak{g}_{2}$ is sent to $\widehat{H}_{\mathcal{C}, Y \cap Z}^{2 s}(X, s)$. We now consider the commutative diagram of complexes

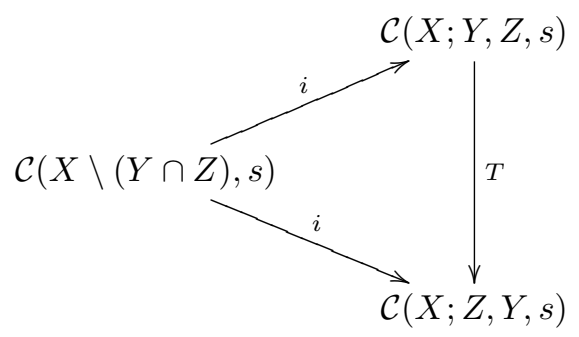

where $T((\alpha, \beta), \gamma)=((\beta, \alpha),-\gamma)$. We note that the isomorphism $T$ induces an isomorphism, also denoted by $T$,

$$
T: \widehat{H}_{\mathcal{C}, Y, Z}^{2 s}(X, s) \stackrel{\cong}{\rightrightarrows} \widehat{H}_{\mathcal{C}, Z, Y}^{2 s}(X, s) .
$$

We are left to compare the element

$$
\begin{aligned}
T\left(\left(\omega_{1}, \widetilde{g}_{1}\right) *\left(\omega_{2}, \widetilde{g}_{2}\right)\right) & =T\left(\omega_{1} \bullet \omega_{2},\left(\left(g_{1} \bullet \omega_{2}, \omega_{1} \bullet g_{2}\right),-g_{1} \bullet g_{2}\right)^{\sim}\right) \\
& =\left(\omega_{1} \bullet \omega_{2},\left(\left(\omega_{1} \bullet g_{2}, g_{1} \bullet \omega_{2}\right), g_{1} \bullet g_{2}\right)^{\sim}\right)
\end{aligned}
$$

with the element

$$
\left(\omega_{2}, \widetilde{g}_{2}\right) *\left(\omega_{1}, \widetilde{g}_{1}\right)=\left(\omega_{2} \bullet \omega_{1},\left(\left(g_{2} \bullet \omega_{1}, \omega_{2} \bullet g_{1}\right),-g_{2} \bullet g_{1}\right)^{\sim}\right)
$$

in the group $\widehat{H}_{\mathcal{C}, Z, Y}^{2 s}(X, s)$. To do this, let us consider the element $y \in \mathcal{C}^{2 s-2}(X ; Z, Y, s)$ given by

$$
y=\left(\left(h_{c}\left(\omega_{1} \otimes g_{2}\right), h_{c}\left(g_{1} \otimes \omega_{2}\right)\right), h_{c}\left(g_{1} \otimes g_{2}\right)\right) .
$$

With this element we derive

$$
T\left(\mathfrak{g}_{1} * \mathfrak{g}_{2}\right)-\mathfrak{g}_{2} * \mathfrak{g}_{1}-(0, \mathrm{~d} y)=\mathrm{a}\left(h_{c}\left(\omega_{1} \otimes \omega_{2}\right)\right) ;
$$

here we have used the fact that the elements $\omega_{j}$ have even degree, and the elements $g_{j}$ have odd degree. This completes the proof of (i). 
$\mathcal{G}$-algebras and $\mathcal{G}$-modules

The above results can be applied to the case of $\mathcal{G}$-algebras and $\mathcal{G}$-modules.

Definition 3.51. A $\mathcal{G}$-algebra over $X$ is a $\mathcal{G}$-complex $\mathcal{C}$ together with a $\mathcal{G}$-pairing

$$
\bullet: \mathcal{C} \otimes \mathcal{C} \rightarrow \mathcal{C}
$$

making $(\mathcal{C}, \bullet)$ into a graded differential algebra with unit element which is associative up to homotopy and commutative up to homotopy. We call $\mathcal{C}$ pseudo-associative or pseudocommutative, if the product $\bullet$ is.

A direct consequence of Theorems 3.42 and 3.49 is the following theorem.

Theorem 3.52. Let $\mathcal{C}$ be a pseudo-associative and pseudo-commutative algebra over $X$. Then, the direct sum

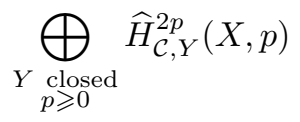

is an associative and commutative algebra.

Once we have the notion of $\mathcal{G}$-algebra, we can define the concept of a $\mathcal{G}$-module.

Definition 3.53. Let $\mathcal{C}$ be a $\mathcal{G}$-algebra over $X$. A $\mathcal{G}$-module over $\mathcal{C}$ is a $\mathcal{G}$-complex $\mathcal{C}^{\prime}$ over $X$ together with a $\mathcal{G}$-pairing

$$
\mathcal{C} \otimes \mathcal{C}^{\prime} \rightarrow \mathcal{C}^{\prime}
$$

which is associative up to homotopy. We call such a pairing a $\mathcal{G}$-action. We call the $\mathcal{G}$-action pseudo-associative, if the pairing is pseudo-associative.

As a consequence of Theorem 3.42, we have the following theorem.

Theorem 3.54. Let $\mathcal{C}$ be a pseudo-associative $\mathcal{G}$-algebra over $X$, and $\mathcal{C}^{\prime}$ a $\mathcal{G}$-complex provided with a pseudo-associative $\mathcal{G}$-action. Then, the direct sum

$$
\bigoplus_{\substack{\text { closed } \\ p \geqslant 0}} \widehat{H}_{\mathcal{C}^{\prime}, Y}^{2 p}(X, p)
$$

is an associative module over the associative algebra $\bigoplus_{Y, p} \widehat{H}_{\mathcal{C}, Y}^{2 p}(X, p)$.

Multiple products

Proposition 3.55. Let $y_{j}$ be $p_{j}$-codimensional cycles of $X, Y_{j}=\operatorname{supp} y_{j}$, and $\mathfrak{g}_{j}$ Green objects for the class of $y_{j}(j=1, \ldots, r)$ such that $\operatorname{codim}\left(Y_{1} \cap \cdots \cap Y_{r}\right)=p_{1}+\cdots+p_{r}$. Then, the $r$-fold $*$-product $\mathfrak{g}_{1} * \cdots * \mathfrak{g}_{r}$ is a well-defined Green object for the class of $y_{1} \cdots y_{r}$ even though partial intersections of the $Y_{j}$ need not be proper.

Proof. The proof of the proposition is an immediate consequence of Theorems 3.37, 3.39 and 3.52 . 


\subsection{Functorial properties of Green objects}

Throughout this section, $k$-scheme will mean regular separated scheme of finite type over $k$.

Direct image of a $\mathcal{G}$-complex

Let $f: X \rightarrow Y$ be a morphism of $k$-schemes, and $\mathcal{C}_{X}$ a $\mathcal{G}$-complex over $X$. Since $\mathcal{C}_{X}$ satisfies the Mayer-Vietoris principle, it is clear that $f_{*} \mathcal{C}_{X}$ also satisfies the MayerVietoris principle. Since $\mathcal{G}$ is a sheaf in the big Zariski site over $k$, there is a morphism of sheaves $\mathcal{G}_{Y} \rightarrow f_{*} \mathcal{G}_{X}$ on $Y$. Therefore, the composition

$$
\mathcal{G}_{Y} \rightarrow f_{*} \mathcal{G}_{X} \rightarrow f_{*} \mathcal{C}_{X}
$$

determines a structure of $\mathcal{G}$-complex on $f_{*} \mathcal{C}_{X}$.

Pull-back of Green objects

Definition 3.56. Let $f: X \rightarrow Y$ be a morphism of $k$-schemes, and let $\mathcal{C}_{X}, \mathcal{C}_{Y}$ be $\mathcal{G}$-complexes over $X, Y$, respectively. A contravariant $f$-morphism (of $\mathcal{G}$-complexes) is a morphism of $\mathcal{G}$-complexes over $Y$ :

$$
f^{\#}: \mathcal{C}_{Y} \rightarrow f_{*} \mathcal{C}_{X}
$$

If $g: Y \rightarrow Z$ is another morphism of $k$-schemes, and $g^{\#}: \mathcal{C}_{Z} \rightarrow g_{*} \mathcal{C}_{Y}$ a contravariant $g$-morphism, then $g_{*}\left(f^{\#}\right) \circ g^{\#}$ is a contravariant $(g \circ f)$-morphism.

Let $f^{\#}$ be as in Definition 3.56. Then, for any open subset $U \subseteq Y$, any closed subset $Z \subseteq U$, and any integer $p$, we have a morphism

$$
s\left(\mathcal{C}_{Y}(U, p), \mathcal{C}_{Y}(U \backslash Z, p)\right) \rightarrow s\left(\mathcal{C}_{X}\left(f^{-1}(U), p\right), \mathcal{C}_{X}\left(f^{-1}(U \backslash Z), p\right)\right)
$$

given by the assignment

$$
(\omega, g) \mapsto\left(f^{\#} \omega, f^{\#} g\right) .
$$

Therefore, we have induced morphisms, again denoted by $f^{\#}$,

$$
\begin{gathered}
f^{\#}: H_{\mathcal{C}_{Y}, Z}^{n}(U, p) \rightarrow H_{\mathcal{C}_{X}, f^{-1}(Z)}^{n}\left(f^{-1}(U), p\right), \\
f^{\#}: \widehat{H}_{\mathcal{C}_{Y}, Z}^{n}(U, p) \rightarrow \widehat{H}_{\mathcal{C}_{X}, f^{-1}(Z)}^{n}\left(f^{-1}(U), p\right) .
\end{gathered}
$$

Theorem 3.57. Let $f: X \rightarrow Y$ be a morphism of $k$-schemes, let $\mathcal{C}_{X}, \mathcal{C}_{Y}$ be $\mathcal{G}$-complexes over $X, Y$, respectively, and let $f^{\#}$ be a contravariant $f$-morphism. Then, we have the following statements.

(i) There is a commutative diagram

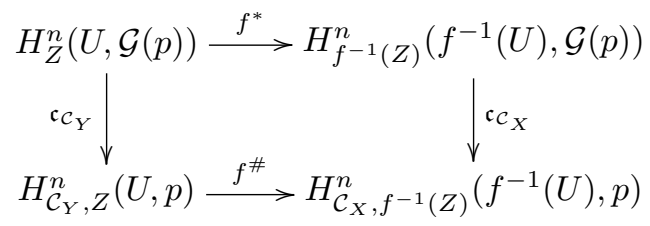


(ii) Setting $U^{\prime}=f^{-1}(U)$ and $Z^{\prime}=f^{-1}(Z)$, we have the commutative diagram

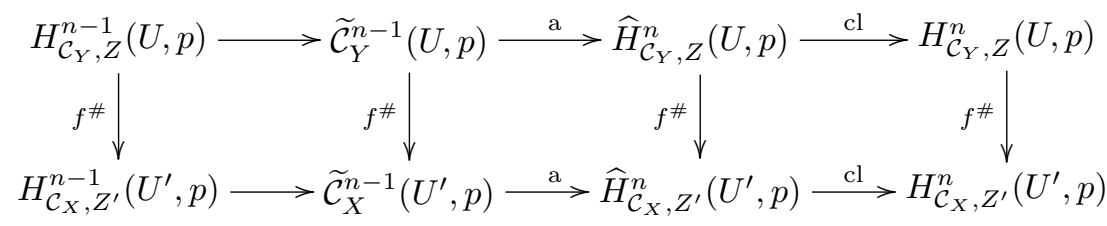

Corollary 3.58. Let $z \in \mathrm{Z}^{p}(Y)$ be a $p$-codimensional cycle on $Y$ with $Z=\operatorname{supp} z$, and $\mathfrak{g}_{z}=\left(\omega_{z}, \widetilde{g}_{z}\right)$ a Green object for the cycle $z$. Then, we have the following.

(i) The element $f^{\#}\left(\mathfrak{g}_{z}\right)$ is a weak Green object for the class of $f^{*}(z)$ in the group $\mathrm{CH}_{f^{-1}(Z)}^{p}(X)$.

(ii) If the cycle $f^{-1}(Z)$ has codimension $p$ in $X$, the element $f^{\#}\left(\mathfrak{g}_{z}\right)$ is a Green object for the cycle $f^{*}(z)$.

By a similar argument to the one used in the course of the proof of Theorem 3.39, we derive the following proposition.

Proposition 3.59. Let $z \in \mathrm{Z}^{p}(Y)$ be a $p$-codimensional cycle on $Y$ with $Z=\operatorname{supp} z$ and codim $f^{-1}(Z)=p$. Let $\mathfrak{g}_{z}$ be a Green object for the class of $z$, and $\mathfrak{g}_{z}^{\prime} \in \widehat{H}_{\mathcal{C}_{Y}, Z}^{2 p}(Y, p)$ be any representative of $\mathfrak{g}_{z}$. Then, the image of $f^{\#}\left(\mathfrak{g}_{z}^{\prime}\right)$ in the group $\widehat{H}_{\mathcal{C}_{X}, \mathcal{Z}^{p}}^{2 p}(X, p)$ is independent of the choice of $\mathfrak{g}_{z}^{\prime}$.

Definition 3.60. With the hypothesis of Proposition 3.59, we will denote the image of $f^{\#}\left(\mathfrak{g}_{z}^{\prime}\right)$ in the group $\widehat{H}_{\mathcal{C}_{X}, \mathcal{Z}^{p}}^{2 p}(X, p)$ by $f^{\#}\left(\mathfrak{g}_{z}\right)$.

Definition 3.61. Let $\mathcal{C}_{X} \otimes \mathcal{C}_{X}^{\prime} \stackrel{\bullet}{\longrightarrow} \mathcal{C}_{X}^{\prime \prime}$, and $\mathcal{C}_{Y} \otimes \mathcal{C}_{Y}^{\prime} \stackrel{\bullet}{\longrightarrow} \mathcal{C}_{Y}^{\prime \prime}$ be $\mathcal{G}$-pairings, and let

$$
\begin{aligned}
& f_{\mathcal{C}}^{\#}: \mathcal{C}_{Y} \rightarrow f_{*} \mathcal{C}_{X}, \\
& f_{\mathcal{C}^{\prime}}^{\#}: \mathcal{C}_{Y}^{\prime} \rightarrow f_{*} \mathcal{C}_{X}^{\prime}, \\
& f_{\mathcal{C}^{\prime \prime}}^{\#}: \mathcal{C}_{Y}^{\prime \prime} \rightarrow f_{*} \mathcal{C}_{X}^{\prime \prime}
\end{aligned}
$$

be contravariant $f$-morphisms. We will call the $f$-morphisms $f_{\mathcal{C}}^{\#}, f_{\mathcal{C}^{\prime}}^{\#}, f_{\mathcal{C}^{\prime \prime}}^{\#}$ compatible with the pairings $\bullet_{X}$ and $\bullet_{Y}$, if the equality

$$
f_{\mathcal{C}}^{\#}(y) \bullet X f_{\mathcal{C}^{\prime}}^{\#}\left(y^{\prime}\right)=f_{\mathcal{C}^{\prime \prime}}^{\#}\left(y \bullet Y y^{\prime}\right)
$$

holds for all sections $y, y^{\prime}$ of $\mathcal{C}_{Y}, \mathcal{C}_{Y}^{\prime}$, respectively.

Proposition 3.62. Let $f_{\mathcal{C}}^{\#}, f_{\mathcal{C}^{\prime}}^{\#}$, and $f_{\mathcal{C}^{\prime \prime}}^{\#}$ be contravariant $f$-morphisms which are compatible with the pairings $\bullet_{X}$ and $\bullet_{Y}$ as in Definition 3.61. Then, for any pair of Green objects $\mathfrak{g}_{1}, \mathfrak{g}_{2}$ the equality

$$
f^{\#}\left(\mathfrak{g}_{1} * \mathfrak{g}_{2}\right)=f^{\#}\left(\mathfrak{g}_{1}\right) * f^{\#}\left(\mathfrak{g}_{2}\right)
$$

holds. 
Change of $\mathcal{G}$-complex

Remark 3.63. We point out that all the results concerning inverse images are compatible with the change of $\mathcal{G}$-complex, i.e. they apply to the case in which we have two $\mathcal{G}$-complexes $\mathcal{C}$ and $\mathcal{C}^{\prime}$ over $X$ together with a morphism $f^{\#}: \mathcal{C} \rightarrow \mathcal{C}^{\prime}$ of $\mathcal{G}$-complexes.

By Corollary 2.63, there is a case, when the change of $\mathcal{G}$-complex does not change the space of Green objects.

Proposition 3.64. Let $X$ be a $k$-scheme, and $\mathcal{C}, \mathcal{C}^{\prime}$ two $\mathcal{G}$-complexes over $X$. Let $f^{\#}$ : $\mathcal{C} \rightarrow \mathcal{C}^{\prime}$ be a morphism of $\mathcal{G}$-complexes such that the morphism

$$
f^{\#}: \mathcal{C}(X, p) \rightarrow \mathcal{C}^{\prime}(X, p)
$$

is an isomorphism for any integer $p$, and such that the morphism

$$
f^{\#}: \mathcal{C}(U, p) \rightarrow \mathcal{C}^{\prime}(U, p)
$$

is a quasi-isomorphism for any open subset $U \subseteq X$ and any integer $p$. Then, the induced morphism

$$
f^{\#}: \widehat{H}_{\mathcal{C}, Z}^{n}(X, p) \rightarrow \widehat{H}_{\mathcal{C}^{\prime}, Z}^{n}(X, p)
$$

is an isomorphism for any closed subset $Z \subseteq X$ and any pair of integers $n, p$.

Proof. This follows immediately from Corollary 2.63 .

Push-forward of Green objects

Definition 3.65. Given a graded complex $A=\left(A^{*}(*), \mathrm{d}\right)$, the twisted complex $A(d)$ is given by $A(d)^{n}(p)=A^{n}(p+d)$ with the same differential.

Definition 3.66. Let $f: X \rightarrow Y$ be a proper morphism of equidimensional $k$-schemes of relative dimension $d$. Let $\mathcal{C}_{X}, \mathcal{C}_{Y}$ be $\mathcal{G}$-complexes over $X, Y$, respectively. A covariant $f$-morphism (of $\mathcal{G}$-complexes) is a morphism of graded complexes of sheaves over $Y$

$$
f_{\#}: f_{*} \mathcal{C}_{X} \rightarrow \mathcal{C}_{Y}(-d)[-2 d]
$$

such that for any open subset $U \subseteq Y$, and any closed subset $Z \subseteq f^{-1}(U)$, the induced diagram

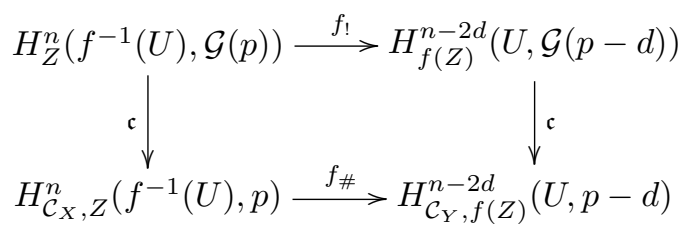

is commutative. In the above diagram, the arrow $f_{\#}$ is given by the composition of morphisms

$$
H_{\mathcal{C}_{X}, Z}^{n}\left(f^{-1}(U), p\right) \rightarrow H_{\mathcal{C}_{X}, f^{-1}(f(Z))}^{n}\left(f^{-1}(U), p\right) \rightarrow H_{\mathcal{C}_{Y}, f(Z)}^{n-2 d}(U, p-d)
$$


here the first map is the restriction morphism, and the second map is induced by the morphism of complexes

$$
s\left(\mathcal{C}_{X}\left(f^{-1}(U), p\right), \mathcal{C}_{X}\left(f^{-1}(U \backslash f(Z)), p\right)\right) \rightarrow s\left(\mathcal{C}_{Y}(U, p-d), \mathcal{C}_{Y}(U \backslash f(Z), p-d)\right)[-2 d]
$$

which is given by the assignment

$$
(\omega, g) \mapsto\left(f_{\#} \omega, f_{\#} g\right)
$$

With the notation and assumptions of Definition 3.66, a covariant $f$-morphism $f_{\#}$ induces a morphism by means of the restriction morphism followed by the morphism of complexes (3.66); this morphism is again denoted $f_{\#}$,

$$
f_{\#}: \widehat{H}_{\mathcal{C}_{X}, Z}^{n}\left(f^{-1}(U), p\right) \rightarrow \widehat{H}_{\mathcal{C}_{Y}, f(Z)}^{n-2 d}(U, p-d) .
$$

If $g: Y \rightarrow Z$ is another proper morphism of equidimensional $k$-schemes of relative dimension $e$, and $g_{\#}: g_{*} \mathcal{C}_{Y} \rightarrow \mathcal{C}_{Z}(-e)[-2 e]$ is a covariant $g$-morphism, then $g_{\#} \circ g_{*}\left(f_{\#}\right)$ is a covariant $(g \circ f)$-morphism.

Theorem 3.68. Let $f: X \rightarrow Y$ be a proper morphism of equidimensional $k$-schemes of relative dimension $d$, and let $f_{\#}$ be a covariant $f$-morphism. Given $U, Z$ as in Definition 3.66, set $U^{\prime}=f^{-1}(U), Z^{\prime}=f(Z)$, respectively. Then, for every $n$, $p$, we have the commutative diagram

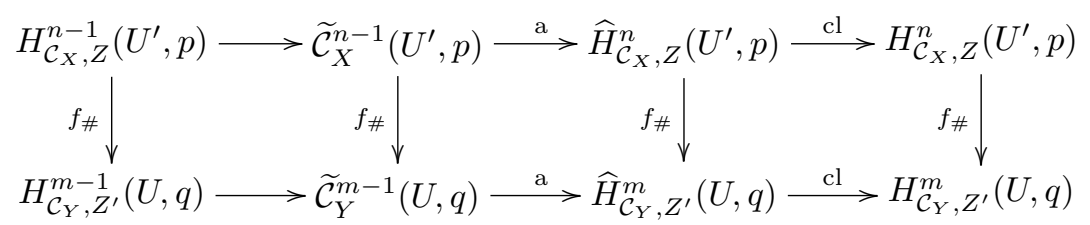

where $q=p-d$ and $m=n-2 d$.

Given a proper morphism $f: X \rightarrow Y$ of equidimensional $k$-schemes of relative dimension $d$, and a closed subset $Z \subseteq X$, we have $\operatorname{codim} f(Z) \geqslant \max (\operatorname{codim} Z-d, 0)$. Therefore, the following result is simpler to prove than the corresponding statement for the inverse image of Green objects.

Proposition 3.69. Let $f: X \rightarrow Y$ be a proper morphism of equidimensional $k$-schemes of relative dimension $d$, and let $f_{\#}$ be a covariant $f$-morphism. Let $z \in Z^{p}(X)$ be a $p$-codimensional cycle on $X$ with $Z=\operatorname{supp} z$. Let $\mathfrak{g}_{z}$ be a Green object for the class of $z$, and $\mathfrak{g}_{z}^{\prime} \in \widehat{H}_{\mathcal{C}_{X}, Z}^{2 p}(X, p)$ be any representative of $\mathfrak{g}_{z}$. Then, the image of $f_{\#}\left(\mathfrak{g}_{z}^{\prime}\right)$ in the group $\widehat{H}_{\mathcal{C}_{Y}, \mathcal{Z}^{p-d}}^{2 p-2 d}(Y, p-d)$ is independent of the choice of $\mathfrak{g}_{z}^{\prime}$; it is a Green object for the class of the cycle $f_{*}(z)$.

Definition 3.70. With the hypothesis of Proposition 3.69, we will denote the image of $f_{\#}\left(\mathfrak{g}_{z}^{\prime}\right)$ in the group $\widehat{H}_{\mathcal{C}_{Y}, \mathcal{Z}^{p-d}}^{2 p-2 d}(Y, p-d)$ by $f_{\#}\left(\mathfrak{g}_{z}\right)$. 


\section{Covariant pseudo-morphisms}

In some cases, the notion of covariant $f$-morphism is too restrictive. Moreover, it is not strictly necessary in order to define direct images of Green objects. Hence, we introduce the notion of covariant $f$-pseudo-morphisms.

Definition 3.71. With the hypothesis of Definition 3.66, a covariant $f$-pseudo-morphism (of $\mathcal{G}$-complexes) is a diagram of morphisms $f_{\#}$ of graded complexes of sheaves over $Y$

$$
f_{*} \mathcal{C}_{X} \stackrel{u}{\longleftarrow} \mathcal{F} \rightarrow \mathcal{C}_{Y}(-d)[-2 d]
$$

where $\mathcal{F}$ is an auxiliary $\mathcal{G}$-complex satisfying the following conditions.

(1) The morphism $u$ is a quasi-isomorphism such that the morphism of global sections

$$
u: \Gamma(Y, \mathcal{F}) \rightarrow \Gamma\left(Y, f_{*} \mathcal{C}_{X}\right)=\Gamma\left(X, \mathcal{C}_{X}\right)
$$

is an isomorphism. Then, as in Definition 3.66, the pseudo-morphism $f_{\#}$ induces a morphism

$$
H_{\mathcal{C}_{X}, Z}^{n}\left(f^{-1}(U), p\right) \stackrel{f_{\#}}{\longrightarrow} H_{\mathcal{C}_{Y}, f(Z)}^{n-2 d}(U, p-d)
$$

for any subset $U \subseteq Y$, and any closed subset $Z \subseteq f^{-1}(U)$.

(2) The diagram

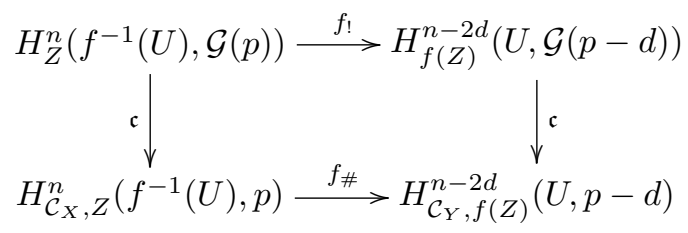

is commutative.

Observe that the definition of a covariant $f$-pseudo-morphism is stronger than the notion of a morphism in the derived category, because we need a well-defined morphism of groups on the level of global sections. Note also that a covariant $f$-morphism determines a covariant $f$-pseudo-morphism by taking for $u$ the identity map.

Since $\mathcal{F}$ and $f_{*} \mathcal{C}_{X}$ satisfy the Mayer-Vietoris principle, they are totally acyclic. Therefore, the fact that $u$ is a quasi-isomorphism of sheaves implies that the induced morphism

$$
\mathcal{F}(U, p) \rightarrow \mathcal{C}_{X}\left(f^{-1}(U), p\right)
$$

is a quasi-isomorphism for every open subset $U \subseteq Y$, and every $p \in \mathbb{Z}$. Taking into account that the induced morphism is an isomorphism on the level of global sections by Corollary 2.63, we obtain the following lemma.

Lemma 3.72. Let $f: X \rightarrow Y$ be a proper morphism of equidimensional $k$-schemes of relative dimension $d$, and let $f_{\#}$ be a covariant $f$-pseudo-morphism. If $Z$ is a closed subset of $X$, the induced morphism

$$
\widehat{H}^{n}(\mathcal{F}(Y, p), \mathcal{F}(Y \backslash f(Z), p)) \rightarrow \widehat{H}^{n}\left(\mathcal{C}_{X}(X, p), \mathcal{C}_{X}\left(X \backslash f^{-1}(f(Z)), p\right)\right)
$$

is an isomorphism. 
This result enables us to define direct images.

Definition 3.73. Let $f: X \rightarrow Y$ be a proper morphism of equidimensional $k$-schemes of relative dimension $d$, and let $f_{\#}$ be a covariant $f$-pseudo-morphism. If $Z$ is a closed subset of $X$, the induced morphism

$$
f_{\#}: \widehat{H}_{\mathcal{C}_{X}, Z}^{n}(X, p) \rightarrow \widehat{H}_{\mathcal{C}_{Y}, f(Z)}^{n-2 d}(Y, p-d)
$$

is defined by the composition

$$
\begin{aligned}
\widehat{H}_{\mathcal{C}_{X}, Z}^{n}(X, p) & =\widehat{H}^{n}\left(\mathcal{C}_{X}(X, p), \mathcal{C}_{X}(X \backslash Z, p)\right) \\
& \rightarrow \widehat{H}^{n}\left(\mathcal{C}_{X}(X, p), \mathcal{C}_{X}\left(X \backslash f^{-1}(f(Z)), p\right)\right) \\
& \cong \widehat{H}^{n}(\mathcal{F}(Y, p), \mathcal{F}(Y \backslash f(Z), p)) \\
& \rightarrow \widehat{H}^{n-2 d}\left(\mathcal{C}_{Y}(Y, p-d), \mathcal{C}_{Y}(Y \backslash f(Z), p-d)\right) \\
& =\widehat{H}_{\mathcal{C}_{Y}, f(Z)}^{n-2 d}(Y, p-d) .
\end{aligned}
$$

Remark 3.74. The analogues of Theorem 3.68 with $U=Y$, and of Proposition 3.69 then also hold for covariant $f$-pseudo-morphisms.

\section{Composition of pseudo-morphisms}

It is not clear from the definition whether we can always define the composition of covariant pseudo-morphisms. Nevertheless, we can determine when a pseudo-morphism should be considered as the composition of two pseudo-morphisms.

For this, let $g: Y \rightarrow Z$ be another proper morphism of equidimensional $k$-schemes of relative dimension $e$, and let $g_{\#}: g_{*} \mathcal{C}_{Y} \stackrel{u^{\prime}}{\longleftarrow} \mathcal{F}^{\prime} \rightarrow \mathcal{C}_{Z}(-e)[-2 e]$ be a covariant $g$-pseudomorphism. Furthermore, let

$$
h_{\#}:(g \circ f)_{*} \mathcal{C}_{X} \stackrel{u^{\prime \prime}}{\longleftarrow} \mathcal{F}^{\prime \prime} \rightarrow \mathcal{C}_{Z}(-d-e)[-2 d-2 e] .
$$

Definition 3.75. We say that $h_{\#}$ is the composition of $f_{\#}$ and $g_{\#}$, if there is a commutative diagram

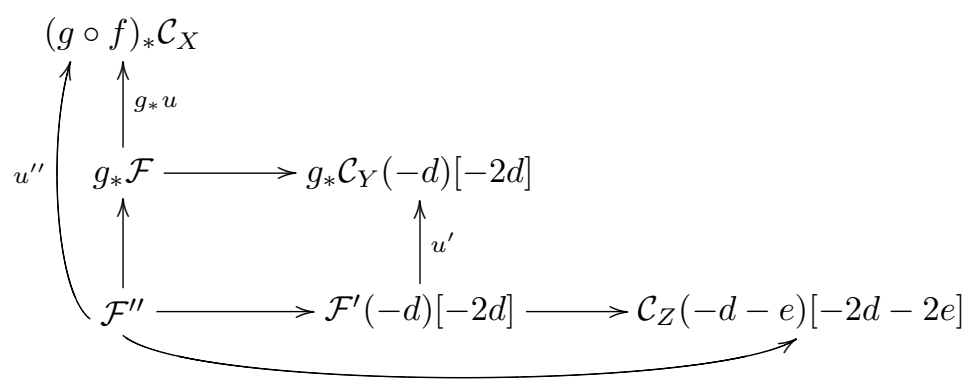


Proposition 3.76. Let $h_{\#}$ be a composition of the pseudo-morphisms $f_{\#}$ and $g_{\#}$. If $W$ is a closed subset of $X$, the induced morphisms on the level of Green objects

$$
\begin{aligned}
f_{\#} & : \widehat{H}_{\mathcal{C}_{X}, W}^{n}(X, p) \rightarrow \widehat{H}_{\mathcal{C}_{Y}, f(W)}^{n-2 d}(Y, p-d), \\
g_{\#} & : \widehat{H}_{\mathcal{C}_{Y}, f(W)}^{n-2 d}(Y, p-d) \rightarrow \widehat{H}_{\mathcal{C}_{Z}, g(f(W))}^{n-2 d-2 e}(Z, p-d-e), \\
h_{\#} & : \widehat{H}_{\mathcal{C}_{X}, W}^{n}(X, p) \rightarrow \widehat{H}_{\mathcal{C}_{Z}, h(W)}^{n-2 d-2 e}(Z, p-d-e)
\end{aligned}
$$

satisfy $h_{\#}=g_{\#} \circ f_{\#}$.

Proof. We may assume that $W$ satisfies $W=h^{-1}(h(W))$. Writing $U=X \backslash W$, $U^{\prime}=Y \backslash f(W), U^{\prime \prime}=Z \backslash h(W)$, and $n^{\prime}=n-2 d, n^{\prime \prime}=n-2 d-2 e$, we obtain the following commutative diagram (where we have omitted the corresponding twists for ease of notation):

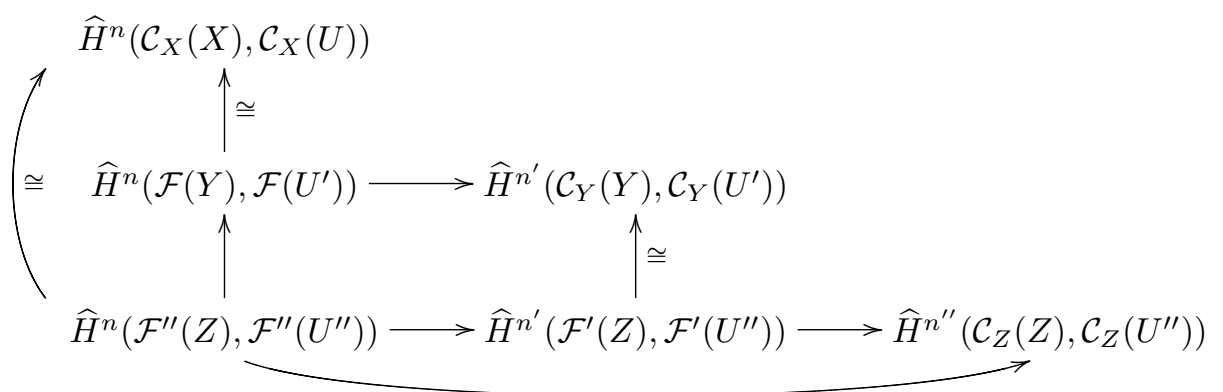

This proves the proposition.

\section{Projection formula}

In order to have a projection formula for Green objects, we need that the complexes under consideration also satisfy a projection formula.

Definition 3.77. Let $f: X \rightarrow Y$ be a proper morphism of equidimensional $k$-schemes of relative dimension $d$. Let $\mathcal{C}_{X}, \mathcal{C}_{X}^{\prime}, \mathcal{C}_{X}^{\prime \prime}$ be $\mathcal{G}$-complexes over $X$, and let $\mathcal{C}_{Y}, \mathcal{C}_{Y}^{\prime}, \mathcal{C}_{Y}^{\prime \prime}$ be $\mathcal{G}$-complexes over $Y$. Let

$$
f^{\#}: \mathcal{C}_{Y} \rightarrow f_{*} \mathcal{C}_{X}
$$

be a contravariant $f$-morphism, and let

$$
f_{\#}^{\prime}: f_{*} \mathcal{C}_{X}^{\prime} \rightarrow \mathcal{C}_{Y}^{\prime}(-d)[-2 d], \quad f_{\#}^{\prime \prime}: f_{*} \mathcal{C}_{X}^{\prime \prime} \rightarrow \mathcal{C}_{Y}^{\prime \prime}(-d)[-2 d]
$$

be covariant $f$-morphisms. Finally, let

$$
\mathcal{C}_{X} \otimes \mathcal{C}_{X}^{\prime} \stackrel{\bullet X}{\longrightarrow} \mathcal{C}_{X}^{\prime \prime}, \quad \mathcal{C}_{Y} \otimes \mathcal{C}_{Y}^{\prime} \stackrel{\bullet}{\longrightarrow} \mathcal{C}_{Y}^{\prime \prime}
$$

be $\mathcal{G}$-pairings. We call $\left(f^{\#}, f_{\#}^{\prime}, f_{\#}^{\prime \prime}, \bullet_{X}, \bullet_{Y}\right)$ a projection five-tuple, if the equality

$$
f_{\#}^{\prime \prime}\left(f^{\#}(y) \bullet X x\right)=y \bullet Y f_{\#}^{\prime}(x)
$$

holds for all sections $x, y$ of $f_{*} \mathcal{C}_{X}^{\prime}, \mathcal{C}_{Y}$, respectively. 
Proposition 3.78. Let $f: X \rightarrow Y$ be a proper morphism of equidimensional $k$-schemes of relative dimension $d$, and let $\left(f^{\#}, f_{\#}^{\prime}, f_{\#}^{\prime \prime}, \bullet_{X}, \bullet_{Y}\right)$ be a projection five-tuple. Then, for any pair of Green objects $\mathfrak{g}_{1}, \mathfrak{g}_{2}$ the equality

$$
f_{\#}^{\prime \prime}\left(f^{\#}\left(\mathfrak{g}_{1}\right) *_{X} \mathfrak{g}_{2}\right)=\mathfrak{g}_{1} *_{Y} f_{\#}^{\prime}\left(\mathfrak{g}_{2}\right)
$$

holds.

Pseudo-morphism and the projection formula

Remark 3.79. Assume that $f_{\#}^{\prime}$ and $f_{\#}^{\prime \prime}$ in Definition 3.77 are only covariant $f$-pseudomorphisms given by

$$
\begin{aligned}
& f_{*} \mathcal{C}_{X}^{\prime} \stackrel{u^{\prime}}{\longleftarrow} \mathcal{F}^{\prime} \stackrel{v^{\prime}}{\longrightarrow} \mathcal{C}_{Y}^{\prime}(-d)[-2 d], \\
& f_{*} \mathcal{C}_{X}^{\prime \prime} \stackrel{u^{\prime \prime}}{\longleftarrow} \mathcal{F}^{\prime \prime} \stackrel{v^{\prime \prime}}{\longrightarrow} \mathcal{C}_{Y}^{\prime \prime}(-d)[-2 d],
\end{aligned}
$$

respectively. In order to have a projection formula on the level of Green objects in this case, we need the following three $\mathcal{G}$-pairings

$$
\mathcal{C}_{X} \otimes \mathcal{C}_{X}^{\prime} \stackrel{\bullet}{\longrightarrow} \mathcal{C}_{X}^{\prime \prime}, \quad \mathcal{C}_{Y} \otimes \mathcal{F}^{\prime} \stackrel{\bullet \mathcal{F}}{\longrightarrow} \mathcal{F}^{\prime \prime}, \quad \mathcal{C}_{Y} \otimes \mathcal{C}_{Y}^{\prime} \stackrel{\bullet}{\longrightarrow} \mathcal{C}_{Y}^{\prime \prime}
$$

satisfying

$$
u^{\prime \prime}\left(y \bullet_{\mathcal{F}} x\right)=f^{\#}(y) \bullet_{X} u^{\prime}(x), \quad v^{\prime \prime}\left(y \bullet_{\mathcal{F}} x\right)=y \bullet_{Y} v^{\prime}(x)
$$

for all sections $x, y$ of $\mathcal{F}^{\prime}, \mathcal{C}_{Y}$, respectively.

\section{Abstract arithmetic Chow groups}

The idea behind the definition of arithmetic Chow groups for a variety $X$ over the ring of integers of a number field is that the variety in question can be 'compactified' by adding the complex variety $X_{\infty}=X \times \mathbb{C}$, or more precisely, by adding a certain cohomology theory on the complex variety $X_{\infty}$. For instance, the cohomology theory involved in the definition of arithmetic Chow groups by Gillet and Soule is the real Deligne-Beilinson cohomology (see [17]). However, the use of secondary characteristic invariants (Green forms, Bott-Chern forms) implies that the arithmetic Chow groups depend not only on the cohomology theory, but also on the particular complex used to compute the cohomology. Therefore, the properties of this complex are reflected in the properties of the arithmetic Chow groups. The objective of this section is to develop an abstract version of the construction of arithmetic Chow rings given in [17] emphasizing how the properties of the complexes under consideration are reflected by the properties of the arithmetic Chow groups.

\subsection{Arithmetic varieties}

In this and the next subsection we will fix frequently used notation. Unless stated otherwise, we will adhere to the notation of $[\mathbf{3 6}]$. For more details we refer the reader to that paper, and also to $[\mathbf{1 7}, \mathbf{7 1}]$. 
Arithmetic rings

Definition 4.1. An arithmetic ring is a triple $\left(A, \Sigma, F_{\infty}\right)$ consisting of an excellent regular noetherian integral domain $A$, a finite non-empty set $\Sigma$ of monomorphisms $\sigma$ : $A \rightarrow \mathbb{C}$, and an antilinear involution $F_{\infty}: \mathbb{C}^{\Sigma} \rightarrow \mathbb{C}^{\Sigma}$ of $\mathbb{C}$-algebras such that the diagram

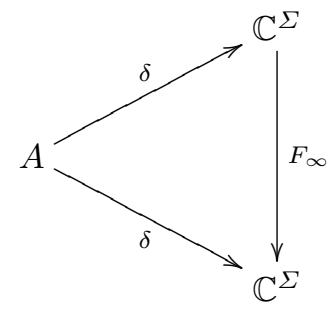

commutes; here $\delta$ is induced by the set of monomorphisms in $\Sigma$.

The main examples of arithmetic rings are subrings of a number field $F$ which have $F$ as fraction field, subrings of $\mathbb{R}$, and $\mathbb{C}$ itself (see $[\mathbf{3 6}, 3.2 .1]$ for details).

\section{Arithmetic varieties}

Notation 4.2. Let $\left(A, \Sigma, F_{\infty}\right)$ be an arithmetic ring with fraction field $F$, and let $X$ be a scheme over $\operatorname{Spec}(A)$. We will write $X_{F}$ for the generic fibre of $X$. If $\sigma \in \Sigma$, we will write $X_{\sigma}=X \underset{\sigma}{\otimes} \mathbb{C}$, and $X_{\Sigma}=X \underset{A}{\otimes} \mathbb{C}^{\Sigma}$. We denote by $X_{\infty}$ the analytic space

$$
X_{\infty}=X_{\Sigma}(\mathbb{C})=\coprod_{\sigma \in \Sigma} X_{\sigma}(\mathbb{C}) .
$$

We observe that the antilinear involution $F_{\infty}$ of $\mathbb{C}^{\Sigma}$ induces an antilinear involution $F_{\infty}$ of $X_{\Sigma}$. We denote the real variety $\left(X_{\Sigma}, F_{\infty}\right)$ by $X_{\mathbb{R}}$.

Let us fix an arithmetic ring $A$, and a Gillet complex $\mathcal{G}$ for schemes over the field of real numbers $\mathbb{R}$.

Definition 4.3. An arithmetic variety $X$ over $A$ is a regular scheme $X$, which is flat and quasi-projective over $\operatorname{Spec}(A)$. If $X$ is equidimensional, we mean by the dimension of $X$ over $A$, the relative dimension of the scheme $X$ over $\operatorname{Spec}(A)$. If $A$ is fixed and clear from the context, we call $X$ simply an arithmetic variety.

Definition 4.4. A $\mathcal{G}$-arithmetic variety over $A$ is a pair

$$
\widehat{X}=(X, \mathcal{C})
$$

consisting of an arithmetic variety $X$ over $A$ and a $\mathcal{G}$-complex $\mathcal{C}$ on the real variety $X_{\mathbb{R}}$.

Definition 4.5. Let $\widehat{X}=\left(X, \mathcal{C}_{X}\right)$ and $\widehat{Y}=\left(Y, \mathcal{C}_{Y}\right)$ be $\mathcal{G}$-arithmetic varieties over $A$. A morphism

$$
f: \widehat{X} \rightarrow \widehat{Y}
$$

of $\mathcal{G}$-arithmetic varieties over $A$ is a pair $f=\left(f, f^{\#}\right)$, where $f: X \rightarrow Y$ is a morphism of $A$-schemes, and $f^{\#}: \mathcal{C}_{Y} \rightarrow f_{*} \mathcal{C}_{X}$ is a contravariant $f_{\mathbb{R}}$-morphism of $\mathcal{G}$-complexes. 
The class of arithmetic varieties over $A$ together with their morphisms forms a category. We denote the category of $\mathcal{G}$-arithmetic varieties over $A$ by $\mathfrak{A}_{A, \mathcal{G}}$. If the cohomology theory $\mathcal{G}$ is fixed, we will write simply $\mathfrak{A}_{A}$ instead of $\mathfrak{A}_{A, \mathcal{G}}$, and we will call a $\mathcal{G}$-arithmetic variety simply an arithmetic variety.

Definition 4.6. A covariant morphism of $\mathcal{G}$-arithmetic varieties

$$
f: \widehat{X}=\left(X, \mathcal{C}_{X}\right) \rightarrow \widehat{Y}=\left(Y, \mathcal{C}_{Y}\right)
$$

over $A$ is a pair $f=\left(f, f_{\#}\right)$, where $f: X \rightarrow Y$ is a proper morphism of equidimensional $A$-schemes of relative dimension $d$, and $f_{\#}: f_{*} \mathcal{C}_{X} \rightarrow \mathcal{C}_{Y}(-d)[-2 d]$ is a covariant $f_{\mathbb{R}}$-morphism of $\mathcal{G}$-complexes. We call $f$ a covariant pseudo-morphism of $\mathcal{G}$-arithmetic varieties over $A$, if $f_{\#}$ is a covariant $f_{\mathbb{R}}$-pseudo-morphism.

Remark 4.7. Let $X$ be an arithmetic variety over $A$. If $\mathcal{C}, \mathcal{C}^{\prime}$ are two $\mathcal{G}$-complexes on $X_{\mathbb{R}}$, and $f: \mathcal{C} \rightarrow \mathcal{C}^{\prime}$ is a morphism of $\mathcal{G}$-complexes, then $f$ can be seen as a morphism of $\mathcal{G}$-arithmetic varieties $\left(X, \mathcal{C}^{\prime}\right) \rightarrow(X, \mathcal{C})$, as well as a covariant morphism of $\mathcal{G}$-arithmetic varieties $(X, \mathcal{C}) \rightarrow\left(X, \mathcal{C}^{\prime}\right)$. Therefore, $f$ will enjoy the properties of both kinds of morphisms.

\subsection{Arithmetic Chow groups}

Throughout this section $\widehat{X}=(X, \mathcal{C})$ will be a $\mathcal{G}$-arithmetic variety over an arithmetic $\operatorname{ring} A$.

\section{Arithmetic cycles}

Notation 4.8. A cycle $y \in \mathrm{Z}^{p}(X)$ on $X$ determines a cycle on $X_{\Sigma}$. Clearly, this cycle is invariant under the action of $F_{\infty}$. Therefore, it is a cycle on $X_{\mathbb{R}}$, which will be denoted by $y_{\mathbb{R}}$. Analogously, any $K_{1}$-chain $f$ on $X$ determines a $K_{1}$-chain $f_{\mathbb{R}}$ on $X_{\mathbb{R}}$.

Since the $\mathcal{G}$-complex $\mathcal{C}$ depends only on $X_{\mathbb{R}}$, we will write as a shorthand

$$
\begin{aligned}
\mathcal{C}^{*}(X, p) & =\mathcal{C}^{*}\left(X_{\mathbb{R}}, p\right), \\
H_{\mathcal{C}, \mathcal{Z}^{p}}^{*}(X, p) & =H_{\mathcal{C}, \mathcal{Z}^{p}}^{*}\left(X_{\mathbb{R}}, p\right), \\
\widehat{H}_{\mathcal{C}, \mathcal{Z}^{p}}^{*}(X, p) & =\widehat{H}_{\mathcal{C}, \mathcal{Z}^{p}}^{*}\left(X_{\mathbb{R}}, p\right),
\end{aligned}
$$

and similar notation for cohomology with supports.

For any cycle $y \in Z^{p}(X)$, we write $\operatorname{cl}(y)$ for the class $\operatorname{cl}_{\mathcal{C}}\left(y_{\mathbb{R}}\right)$. For any $K_{1}$-chain $f \in$ $R_{p}^{p-1}(X)$, we write $\operatorname{cl}(f)$ for the class $\operatorname{cl}_{\mathcal{C}}\left(f_{\mathbb{R}}\right)$, and $\mathfrak{g}(f)=\mathfrak{g}\left(f_{\mathbb{R}}\right)$ (see Definition 3.24).

Definition 4.9. We define the group of $p$-codimensional arithmetic cycles of $\widehat{X}=(X, \mathcal{C})$ by

$$
\widehat{\mathrm{Z}}^{p}(X, \mathcal{C})=\left\{\left(y, \mathfrak{g}_{y}\right) \in \mathrm{Z}^{p}(X) \oplus \widehat{H}_{\mathcal{C}, \mathcal{Z}^{p}}^{2 p}(X, p) \mid \operatorname{cl}(y)=\operatorname{cl}\left(\mathfrak{g}_{y}\right)\right\}
$$

with the obvious group structure. 
Rational equivalence

We now define rational equivalence in this set-up.

Definition 4.10. Let $f \in R_{p}^{p-1}(X)$ be a $K_{1}$-chain. We set

$$
\widehat{\operatorname{div}}(f)=(\operatorname{div}(f), \mathfrak{g}(f)) .
$$

By Remark 3.25, we have $\widehat{\operatorname{div}}(f) \in \widehat{\mathrm{Z}}^{p}(X, \mathcal{C})$. We define

$$
\widehat{\operatorname{Rat}}^{p}(X, \mathcal{C})=\left\{\widehat{\operatorname{div}}(f) \mid f \in R_{p}^{p-1}(X)\right\} \subseteq \widehat{\mathrm{Z}}^{p}(X, \mathcal{C}) .
$$

It is easy to see that $\widehat{\operatorname{Rat}}^{p}(X, \mathcal{C})$ is a subgroup of $\widehat{Z}^{p}(X, \mathcal{C})$.

Definition and basic properties

Definition 4.11. The pth arithmetic Chow group of $\widehat{X}=(X, \mathcal{C})$ is defined by

$$
\widehat{\mathrm{CH}}^{p}(X, \mathcal{C})=\widehat{\mathrm{Z}}^{p}(X, \mathcal{C}) / \widehat{\operatorname{Rat}}^{p}(X, \mathcal{C}) .
$$

The groups $\widehat{\mathrm{CH}}^{p}(X, \mathcal{C})$ will also be called $\mathcal{C}$-arithmetic Chow groups of $X$. The class of the arithmetic cycle $\left(y, \mathfrak{g}_{y}\right)$ in $\widehat{\mathrm{CH}}^{p}(X, \mathcal{C})$ will be denoted by $\left[y, \mathfrak{g}_{y}\right]$.

Notation 4.12. There are well-defined maps

$$
\begin{array}{ll}
\zeta: \widehat{\mathrm{CH}}^{p}(X, \mathcal{C}) \rightarrow \mathrm{CH}^{p}(X), & \zeta\left[y, \mathfrak{g}_{y}\right]=[y], \\
\rho: \mathrm{CH}^{p, p-1}(X) \rightarrow H_{\mathcal{C}}^{2 p-1}(X, p) \subseteq \widetilde{\mathcal{C}}^{2 p-1}(X, p), & \rho[f]=\operatorname{cl}(f), \\
\mathrm{a}: \widetilde{\mathcal{C}}^{2 p-1}(X, p) \rightarrow \widehat{\mathrm{CH}}^{p}(X, \mathcal{C}), & \mathrm{a}(\widetilde{a})=[0, \mathrm{a}(\widetilde{a})], \\
\omega: \widehat{\mathrm{CH}}^{p}(X, \mathcal{C}) \rightarrow \mathrm{ZC} \mathcal{C}^{2 p}(X, p), & \omega\left[y, \mathfrak{g}_{y}\right]=\omega\left(\mathfrak{g}_{y}\right), \\
h: \mathrm{ZC}^{2 p}(X, p) \rightarrow H_{\mathcal{C}}^{2 p}(X, p), & h(\alpha)=[\alpha] .
\end{array}
$$

We will also write

$$
\begin{aligned}
\widehat{\mathrm{CH}}^{p}(X, \mathcal{C})_{0} & =\operatorname{Ker}\left(\omega: \widehat{\mathrm{CH}}^{p}(X, \mathcal{C}) \rightarrow \mathrm{ZC}^{2 p}(X, p)\right), \\
\mathrm{CH}^{p}(X)_{0} & =\operatorname{Ker}\left(\mathrm{cl}: \mathrm{CH}^{p}(X) \rightarrow H_{\mathcal{C}}^{2 p}(X, p)\right) .
\end{aligned}
$$

Theorem 4.13. Using the notation following Definition 3.12, we have the following exact sequences:

$$
\begin{aligned}
& \mathrm{CH}^{p-1, p}(X) \stackrel{\rho}{\rightarrow} \widetilde{\mathcal{C}}^{2 p-1}(X, p)^{\text {pure }} \stackrel{\text { a }}{\rightarrow} \widehat{\mathrm{CH}}^{p}(X, \mathcal{C}) \stackrel{\zeta}{\rightarrow} \mathrm{CH}^{p}(X) \rightarrow 0, \\
& \mathrm{CH}^{p-1, p}(X) \stackrel{\rho}{\rightarrow} H_{\mathcal{C}}^{2 p-1}(X, p)^{\text {pure }} \stackrel{\text { a }}{\rightarrow} \widehat{\mathrm{CH}}^{p}(X, \mathcal{C}) \\
& \qquad \stackrel{(\zeta,-\omega)}{\rightarrow} \mathrm{CH}^{p}(X) \oplus \mathrm{ZC}^{2 p}(X, p) \stackrel{\text { cl }+h}{\rightarrow} H_{\mathcal{C}}^{2 p}(X, p) \rightarrow 0, \\
& \mathrm{CH}^{p-1, p}(X) \stackrel{\rho}{\rightarrow} H_{\mathcal{C}}^{2 p-1}(X, p)^{\text {pure }} \stackrel{\text { a }}{\rightarrow} \widehat{\mathrm{CH}}^{p}(X, \mathcal{C})_{0} \stackrel{\zeta}{\rightarrow} \mathrm{CH}^{p}(X)_{0} \rightarrow 0 .
\end{aligned}
$$


Proof. Let us prove the exactness of the first sequence. By Proposition 3.23, for any $p$-codimensional cycle $y$ on $X$ there exists a Green object $\mathfrak{g}_{y}$ for the class of $y$. Therefore, $\zeta$ is surjective.

It is clear that $\zeta \circ \mathrm{a}=0$. On the other hand, assume that $\zeta(\alpha)=0$, and let $\left(y, \mathfrak{g}_{y}\right)$ be any representative of $\alpha$. Then, we have $y=\operatorname{div}(f)$ for some $K_{1}$-chain $f$, and we obtain $\left(y, \mathfrak{g}_{y}\right) \sim\left(0, \mathfrak{g}_{y}-\mathfrak{g}(f)\right)$. Since $\mathfrak{g}_{y}-\mathfrak{g}(f)$ is a Green object for the trivial cycle, we have $\operatorname{cl}\left(\mathfrak{g}_{y}-\mathfrak{g}(f)\right)=0$. By the exact sequence (3.15), we find $\mathfrak{g}_{y}-\mathfrak{g}(f) \in \mathrm{a}\left(\widetilde{\mathcal{C}}^{2 p-1}(X, p)^{\text {pure }}\right)$, i.e. $\alpha \in \operatorname{Im}(\mathrm{a})$.

Recalling $\mathfrak{g}(f)=\mathrm{b}(\operatorname{cl}(f))$ together with equation (2.55), we find $\mathfrak{g}(f)=\mathrm{a}(\operatorname{cl}(f))$, which shows a $\circ \rho=0$. On the other hand, if $\mathrm{a}(\widetilde{a})=0$, we have $(0, \mathrm{a}(\widetilde{a}))=\widehat{\operatorname{div}}(f)$ for some $K_{1}$-chain $f$. Therefore, we have $\operatorname{div}(f)=0$, and $\mathfrak{g}(f)=\mathrm{a}(\widetilde{a})$. Since $\operatorname{div}(f)=0$, we have $\operatorname{cl}(f) \in H_{\mathcal{C}}^{2 p-1}(X, p)$, from which we derive as before $\mathfrak{g}(f)=\mathrm{b}(\operatorname{cl}(f))=\mathrm{a}(\operatorname{cl}(f))$. Therefore, $\widetilde{a}-\operatorname{cl}(f)$ lies in the kernel of a. By the exact sequence (3.15), this implies $\widetilde{a}=\operatorname{cl}(f)=\rho[f]$.

The proof of the other two exact sequences follows the same lines, but uses the exact sequence (3.16) instead.

Note that at this abstract level, the above theorem is a formal consequence of the definitions.

\subsection{Arithmetic intersection pairing}

The aim of this subsection is to define an arithmetic intersection pairing. We will follow the strategy of $[\mathbf{3 6}]$. Therefore, we divide out by finite rational equivalence before taking Green objects into account.

Finite support

Definition 4.17. Let fin, respectively $\tau(p)$, be the following families of supports:

$$
\text { fin }=\left\{Y \subseteq X \mid Y \text { closed, } Y \cap X_{F}=\emptyset\right\},
$$

respectively

$$
\tau(p)=\left\{Y \subseteq X \mid Y \text { closed, } \operatorname{codim}\left(Y \cap X_{F}\right) \geqslant p\right\}
$$

With this notation, we have the exact sequences

$$
\begin{aligned}
& \bigoplus_{\substack{x \in X^{(p-1)} \\
\{x\} \cap X_{F}=\emptyset}} k(x)^{*} \stackrel{\text { div }}{\longrightarrow} \mathrm{Z}^{p}(X) \rightarrow \mathrm{Z}^{p}\left(X_{F}\right) \oplus \mathrm{CH}_{\mathrm{fin}}^{p}(X) \rightarrow 0, \\
& \bigoplus_{x \in X_{F}^{(p-1)}} k(x)^{*} \stackrel{\text { div }}{\longrightarrow} \mathrm{Z}^{p}\left(X_{F}\right) \oplus \mathrm{CH}_{\mathrm{fin}}^{p}(X) \rightarrow \mathrm{CH}^{p}(X) \rightarrow 0,
\end{aligned}
$$

and a canonical isomorphism

$$
\mathrm{CH}_{\tau(p)}^{p}(X) \cong \mathrm{Z}^{p}\left(X_{F}\right) \oplus \mathrm{CH}_{\mathrm{fin}}^{p}(X) .
$$


Moreover, if $x \in X^{(p-1)}$ with $\overline{\{x\}} \cap X_{F}=\emptyset$, and $f \in k(x)^{*}$, we have $\operatorname{div}(f) \cap X_{F}=\emptyset$, and $\mathfrak{g}(f)=0$. Therefore, we find

$$
\widehat{\operatorname{div}}(f)=(\operatorname{div}(f), 0) .
$$

Thus, for any $\mathcal{G}$-arithmetic variety, there is a short exact sequence

$$
\bigoplus_{x \in X_{F}^{(p-1)}} k(x)^{*} \stackrel{\widehat{\mathrm{div}}}{\longrightarrow} \widehat{\mathrm{Z}}^{p}\left(X_{F}, \mathcal{C}\right) \oplus \mathrm{CH}_{\mathrm{fin}}^{p}(X) \rightarrow \widehat{\mathrm{CH}}^{p}(X, \mathcal{C}) \rightarrow 0
$$

Observe that the notation $\widehat{\mathrm{Z}}^{p}\left(X_{F}, \mathcal{C}\right)$ makes sense, since $\left(X_{F}, \mathcal{C}\right)$ is a $\mathcal{G}$-arithmetic variety over the arithmetic ring $F$.

\section{The intersection pairing}

Let $y$ be a $p$-codimensional, and $z$ a $q$-codimensional cycle of $X$. Put $Y=\operatorname{supp} y$, and $Z=\operatorname{supp} z$. Assume that $y_{F}$ and $z_{F}$ intersect properly; this shows $Y \cap Z \in \tau(p+q)$. By Theorem 1.33, there is a well-defined class

$$
[y] \cdot[z] \in \mathrm{CH}_{Y \cap Z}^{p+q}(X)_{\mathbb{Q}},
$$

and hence a well-defined class

$$
[y] \cdot[z] \in \mathrm{CH}_{\tau(p+q)}^{p+q}(X)_{\mathbb{Q}} \cong \mathrm{Z}^{p+q}\left(X_{F}\right)_{\mathbb{Q}} \oplus \mathrm{CH}_{\text {fin }}^{p+q}(X)_{\mathbb{Q}} .
$$

Let $(X, \mathcal{C}),\left(X, \mathcal{C}^{\prime}\right),\left(X, \mathcal{C}^{\prime \prime}\right)$ be three $\mathcal{G}$-arithmetic varieties with the same underlying scheme $X$, and let $\bullet: \mathcal{C} \otimes \mathcal{C}^{\prime} \rightarrow \mathcal{C}^{\prime \prime}$ be a $\mathcal{G}$-pairing. Let $\mathfrak{g}_{y}$ be a Green object for the class of $y$ with values in $\mathcal{C}$, and $\mathfrak{g}_{z}$ a Green object for the class of $z$ with values in $\mathcal{C}^{\prime}$. Then, the $*$-product $\mathfrak{g}_{y} * \mathfrak{g}_{z}$ is a Green object for the class of $y_{F} \cdot z_{F}$ with values in $\mathcal{C}^{\prime \prime}$ (see Definition 3.40), and we write

$$
\left(y, \mathfrak{g}_{y}\right) \cdot\left(z, \mathfrak{g}_{z}\right)=\left(\left(y_{F} \cdot z_{F}, \mathfrak{g}_{y} * \mathfrak{g}_{z}\right),[y \cdot z]_{\mathrm{fin}}\right) \in \widehat{\mathrm{Z}}^{p+q}\left(X_{F}, \mathcal{C}^{\prime \prime}\right)_{\mathbb{Q}} \oplus \mathrm{CH}_{\mathrm{fin}}^{p+q}(X)_{\mathbb{Q}}
$$

We will denote the image of the product $\left(y, \mathfrak{g}_{y}\right) \cdot\left(z, \mathfrak{g}_{z}\right)$ in $\widehat{\mathrm{CH}}^{p+q}\left(X, \mathcal{C}^{\prime \prime}\right)_{\mathbb{Q}}$ by $\left[\left(y, \mathfrak{g}_{y}\right) \cdot\right.$ $\left.\left(z, \mathfrak{g}_{z}\right)\right]$.

Theorem 4.19. Let $(X, \mathcal{C}),\left(X, \mathcal{C}^{\prime}\right),\left(X, \mathcal{C}^{\prime \prime}\right)$ be three $\mathcal{G}$-arithmetic varieties with the same underlying scheme $X$, and let $\bullet: \mathcal{C} \otimes \mathcal{C}^{\prime} \rightarrow \mathcal{C}^{\prime \prime}$ be a $\mathcal{G}$-pairing. Then, we have the following statements.

(i) There exists a pairing

$$
\widehat{\mathrm{CH}}^{p}(X, \mathcal{C}) \otimes \widehat{\mathrm{CH}}^{q}\left(X, \mathcal{C}^{\prime}\right) \dot{\rightarrow} \widehat{\mathrm{CH}}^{p+q}\left(X, \mathcal{C}^{\prime \prime}\right)_{\mathbb{Q}},
$$

which is determined by formula (4.17) for cycles intersecting properly in the generic fibre. 
(ii) The following diagrams commute:

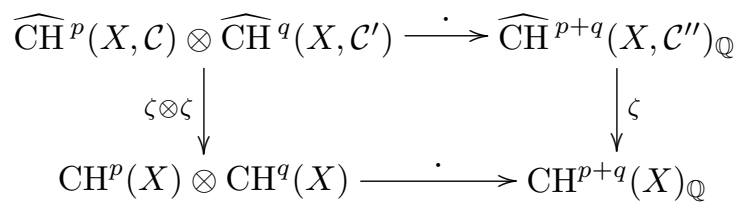

and

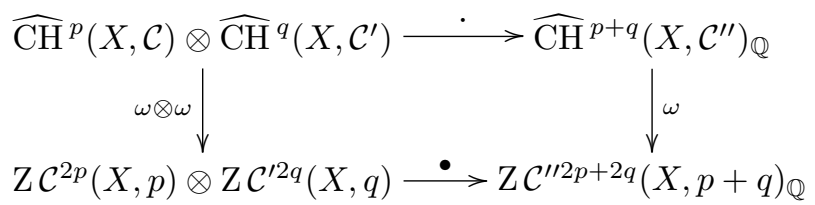

Proof. Let $\alpha \in \widehat{\mathrm{CH}}^{p}(X, \mathcal{C})$, and $\beta \in \widehat{\mathrm{CH}}^{q}\left(X, \mathcal{C}^{\prime}\right)$. By the moving lemma for cycles on a regular variety over a field, there are representatives $\left(y, \mathfrak{g}_{y}\right)$ of $\alpha$, and $\left(z, \mathfrak{g}_{z}\right)$ of $\beta$ such that $y_{F}$ and $z_{F}$ intersect properly. By means of formula (4.17), we define

$$
\alpha \cdot \beta=\left[\left(y, \mathfrak{g}_{y}\right) \cdot\left(z, \mathfrak{g}_{z}\right)\right] .
$$

We have to show that this definition does not depend on the choice of representatives. Let $\left(y^{\prime}, \mathfrak{g}_{y^{\prime}}^{\prime}\right)$ be another representative of $\alpha$ such that $y_{F}^{\prime}$ and $z_{F}$ also intersect properly. Then, there exists a $K_{1}$-chain $f$ such that

$$
\widehat{\operatorname{div}}(f)=\left(y^{\prime}, \mathfrak{g}_{y^{\prime}}^{\prime}\right)-\left(y, \mathfrak{g}_{y}\right) .
$$

Therefore, it suffices to show that whenever $f$ is a $K_{1}$-chain whose $\operatorname{divisor} \operatorname{div}(f)_{F}$ intersects $z_{F}$ properly, then there exists a $K_{1}$-chain $g$ such that

$$
\widehat{\operatorname{div}}(g)=\widehat{\operatorname{div}}(f) \cdot\left(z, \mathfrak{g}_{z}\right) \in \widehat{\mathrm{Z}}^{p+q}\left(X_{F}, \mathcal{C}^{\prime \prime}\right)_{\mathbb{Q}} \oplus \mathrm{CH}_{\mathrm{fin}}^{p+q}(X)_{\mathbb{Q}} \cdot
$$

Let $S=\operatorname{supp} \operatorname{div}(f), U=X \backslash S$, and $Z=\operatorname{supp} z$. By Corollary 1.35 and Proposition 1.55, there exists a $K_{1}$-chain $g \in R_{p+q}^{p+q-1}(X)_{\mathbb{Q}}$ satisfying

$$
\begin{aligned}
{[\operatorname{div}(g)] } & =[\operatorname{div}(f)] \cdot[z] \in \mathrm{CH}_{S \cap Z}^{p+q}(X)_{\mathbb{Q}}, \\
\operatorname{cl}(g) & =\operatorname{cl}(f) \bullet \operatorname{cl}(z) \in H_{\mathcal{C}^{\prime \prime}, U \cap Z}^{2 p+2 q-1}(X \backslash(S \cap Z), p+q)_{\mathbb{Q}} .
\end{aligned}
$$

Since $S \cap Z$ lies in $\tau(p+q)$, equation (4.19) is also valid in $\mathrm{CH}_{\tau(p+q)}^{p+q}(X)_{\mathbb{Q}}$ which shows

$$
[\operatorname{div}(g)]=[\operatorname{div}(f)] \cdot[z]=\left(\operatorname{div}(f)_{F} \cdot z_{F},[\operatorname{div}(f) \cdot z]_{\text {fin }}\right) .
$$

Furthermore, we derive from equation (4.20) and Proposition 2.67

$$
\mathfrak{g}(g)=\mathrm{b}(\operatorname{cl}(g))=\mathrm{b}(\operatorname{cl}(f) \bullet \operatorname{cl}(z))=\mathrm{b}(\operatorname{cl}(f)) * \mathfrak{g}_{z}=\mathfrak{g}(f) * \mathfrak{g}_{z} .
$$

Summing up, we find the equality

$$
\widehat{\operatorname{div}}(g)=\left(\left(\operatorname{div}(f)_{F} \cdot z_{F}, \mathfrak{g}(f) * \mathfrak{g}_{z}\right),[\operatorname{div}(f) \cdot z]_{\operatorname{fin}}\right)=\widehat{\operatorname{div}}(f) \cdot\left(z, \mathfrak{g}_{z}\right)
$$

in the group $\widehat{\mathrm{Z}}^{p+q}\left(X_{F}, \mathcal{C}^{\prime \prime}\right)_{\mathbb{Q}} \oplus \mathrm{CH}_{\text {fin }}^{p+q}(X)_{\mathbb{Q}}$, as desired.

The compatibility of the product with the morphisms $\zeta$ and $\omega$ follows directly from the definitions. 
Remark 4.22. It may be interesting to explain more clearly why we do not need the $K_{1}$ chain moving lemma. A close look at the proof of the well-definedness of the arithmetic intersection product in [36] shows that the $K_{1}$-chain moving lemma is used, first, to define a $K_{1}$-chain up to boundaries $f \cdot[z]$ (which is the $g$ we are using in our proof) and second, to prove two compatibility properties

$$
\begin{aligned}
\operatorname{div}(f \cdot[z]) & =\operatorname{div}(f) \cdot[z], \\
\log |f \cdot[z]|^{2} & =\log |f|^{2} \wedge \delta_{z} .
\end{aligned}
$$

We observe that in the proof, the $K_{1}$-chain $f \cdot[z]$ and the first compatibility condition is used on the whole arithmetic variety $X$ where no $K_{1}$-chain moving lemma is available. Therefore, a result similar to Corollary 1.35 is used implicitly. This means that the essential use of the moving lemma for $K_{1}$-chains is to prove the compatibility with Green currents: it is the concrete nature of the Green currents that forces the use of the $K_{1}$-chain moving lemma.

In our case the compatibility that we need is equation (4.20), which follows directly from the properties of a Gillet cohomology. Roughly speaking, since the map that sends a $K_{1}$-chain to the associated Green object factors through a cohomology group, we are able to avoid the $K_{1}$-chain moving lemma.

The fact that the product is defined only after tensoring with $\mathbb{Q}$ is due to the lack of a satisfactory intersection theory on general regular schemes, and the use of $K$-theory. In the cases in which an intersection product of cycles can be defined without using $K$-theory, it is possible to work over the integers. For instance, this is the case when $y$ and $z$ intersect properly on the whole of $X$, and not just on $X_{F}$; then, the product $y \cdot z$ is a well-defined class in $\mathrm{CH}_{Y \cap Z}^{p+q}(X)$. Therefore, formula (4.17) gives rise to an element of $\widehat{Z}^{p+q}\left(X, \mathcal{C}^{\prime \prime}\right) \oplus \mathrm{CH}^{p+q}(X)$. In particular, by the same argument as in [36, 4.2.3.iii] one can prove the following refinement of Theorem 4.19.

Theorem 4.23. If $p=1$, or $q=1$, there is a unique pairing

$$
\widehat{\mathrm{CH}}^{p}(X, \mathcal{C}) \otimes \widehat{\mathrm{CH}}^{q}\left(X, \mathcal{C}^{\prime}\right) \stackrel{\rightarrow}{\rightarrow} \widehat{\mathrm{CH}}^{p+q}\left(X, \mathcal{C}^{\prime \prime}\right)
$$

given by formula (4.17) when the cycles intersect properly. Moreover, this pairing induces the pairing of Theorem 4.19.

\section{$\mathcal{G}$-algebras}

Let us consider the case when $(X, \mathcal{C})$ is a $\mathcal{G}$-arithmetic variety with $\mathcal{C}$ being a $\mathcal{G}$-algebra.

Theorem 4.24. Let $(X, \mathcal{C})$ be a $\mathcal{G}$-arithmetic variety, and assume that $\mathcal{C}$ is a pseudoassociative and pseudo-commutative $\mathcal{G}$-algebra. Then, we have the following statements.

(i) $\bigoplus_{p} \widehat{\mathrm{CH}}^{p}(X, \mathcal{C})_{\mathbb{Q}}$ is a commutative and associative $\mathbb{Q}$-algebra with unit.

(ii) For $\alpha \in \widehat{\mathrm{CH}}^{1}(X, \mathcal{C}), \beta \in \widehat{\mathrm{CH}}^{1}(X, \mathcal{C})$, and $\gamma \in \widehat{\mathrm{CH}}^{p}(X, \mathcal{C})$, we have

$$
\begin{aligned}
\alpha \cdot \gamma & =\gamma \cdot \alpha \in \widehat{\mathrm{CH}}^{p+1}(X, \mathcal{C}), \\
(\alpha \cdot \beta) \cdot \gamma & =\alpha \cdot(\beta \cdot \gamma) \in \widehat{\mathrm{CH}}^{p+2}(X, \mathcal{C}) .
\end{aligned}
$$


(iii) $\bigoplus_{p} \widehat{\mathrm{CH}}^{p}(X, \mathcal{C})_{0, \mathbb{Q}}=(\operatorname{Ker} \omega)_{\mathbb{Q}}$ is an ideal of $\bigoplus_{p} \widehat{\mathrm{CH}}^{p}(X, \mathcal{C})_{\mathbb{Q}}$.

(iv) $(\operatorname{Ker} \zeta)_{\mathbb{Q}}$ is an ideal of $\bigoplus_{p} \widehat{\mathrm{CH}}^{p}(X, \mathcal{C})_{0, \mathbb{Q}}$.

Proof. Since the product of algebraic cycles is commutative and associative, the first two statements of the theorem follow from the commutativity and associativity of the *-product of Green objects (see Theorems 3.49 and 3.42). The last two statements are immediate.

Theorem 4.25. Let $(X, \mathcal{C})$ be a $\mathcal{G}$-arithmetic variety, and assume that $\mathcal{C}$ is a pseudoassociative and pseudo-commutative $\mathcal{G}$-algebra. The $\bigoplus_{p} \widehat{\mathrm{CH}}^{p}(X, \mathcal{C})_{\mathbb{Q}}$-module structure of $\bigoplus_{p} \widehat{\mathrm{CH}}^{p}(X, \mathcal{C})_{0, \mathbb{Q}}$ is then induced by a $\bigoplus_{p} \mathrm{CH}^{p}(X)_{\mathbb{Q}}$-module structure, i.e. there is a commutative diagram

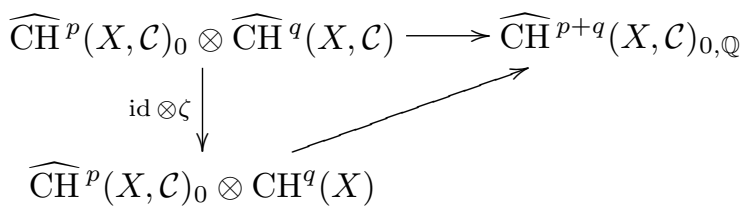

Proof. We have to show that $\operatorname{Ker}(\zeta) \cdot \operatorname{Im}(\mathrm{a})=0$. This follows from Proposition 2.67 (i).

Corollary 4.26. With the assumptions of Theorem 4.25 there is a well-defined product

$$
\mathrm{CH}^{p}(X)_{0} \otimes \mathrm{CH}^{q}(X)_{0} \dot{\rightarrow} \widehat{\mathrm{CH}}^{p+q}(X, \mathcal{C})_{0, \mathbb{Q}}
$$

Proof. Let $x \in \mathrm{CH}^{p}(X)_{0}$ and let $y \in \mathrm{CH}^{q}(X)_{0}$. We choose arithmetic cycles $\widehat{x} \in$ $\widehat{\mathrm{CH}}^{p}(X, \mathcal{C})_{0}$, respectively $\widehat{y} \in \widehat{\mathrm{CH}}^{q}(X, \mathcal{C})_{0}$ such that $\zeta(\widehat{x})=x$, respectively $\zeta(\widehat{y})=y$. By Theorem 4.25, the product $\widehat{x} \cdot \widehat{y}$ does not depend on the choice of $\widehat{x}$ and $\widehat{y}$.

Remark 4.27. If $\mathcal{C}$ is only a $\mathcal{G}$-algebra, then the product in $\widehat{\mathrm{CH}}^{*}(X, \mathcal{C})_{\mathbb{Q}}$ does not need to be associative or commutative. Nevertheless, Theorems 3.42 and 3.49 imply that the product induced in $\bigoplus_{p} \widehat{\mathrm{CH}}^{*}(X, \mathcal{C})_{0, \mathbb{Q}}$ is associative and commutative.

\subsection{Inverse images}

Let $f:\left(X, \mathcal{C}_{X}\right) \rightarrow\left(Y, \mathcal{C}_{Y}\right)$ be a morphism of $\mathcal{G}$-arithmetic varieties. In this section we will construct a pull-back morphism for arithmetic Chow groups.

Definition and basic properties of inverse images

Let $\left(z, \mathfrak{g}_{z}\right) \in \widehat{\mathrm{Z}}^{p}\left(Y, \mathcal{C}_{Y}\right)$ be a $p$-codimensional arithmetic cycle such that $f^{-1}\left(\operatorname{supp} z_{\mathbb{R}}\right)$ has codimension $p$ in $X_{\mathbb{R}}$. By Theorem 1.38, there is a well-defined cycle $f^{*}(z) \in$ $\mathrm{CH}_{\tau(p)}^{p}(X)$. Moreover, by Definition 3.60 , there is a well-defined Green object $f^{\#}\left(\mathfrak{g}_{z}\right)$ for the class of $f^{*}\left(z_{\mathbb{R}}\right)$. In this case we will write

$$
f^{*}\left(z, \mathfrak{g}_{z}\right)=\left(f^{*}(z), f^{\#}\left(\mathfrak{g}_{z}\right)\right) .
$$


Theorem 4.29. Let $f:\left(X, \mathcal{C}_{X}\right) \rightarrow\left(Y, \mathcal{C}_{Y}\right)$ be a morphism of $\mathcal{G}$-arithmetic varieties. Then, the following statements hold.

(i) There is a well-defined morphism of graded groups

$$
f^{*}: \widehat{\mathrm{CH}}^{p}\left(Y, \mathcal{C}_{Y}\right) \rightarrow \widehat{\mathrm{CH}}^{p}\left(X, \mathcal{C}_{X}\right)
$$

induced by equation (4.27) for arithmetic cycles $\left(z, \mathfrak{g}_{z}\right) \in \widehat{\mathrm{Z}}^{p}\left(Y, \mathcal{C}_{Y}\right)$ such that $f^{-1}\left(\operatorname{supp} z_{\mathbb{R}}\right)$ has codimension $p$ in $X_{\mathbb{R}}$.

(ii) If $g:\left(Y, \mathcal{C}_{Y}\right) \rightarrow\left(Z, \mathcal{C}_{Z}\right)$ is another morphism of $\mathcal{G}$-arithmetic varieties, then the equality $(g \circ f)^{*}=f^{*} \circ g^{*}$ holds.

(iii) The pull-back of arithmetic cycles satisfies the following relations:

$$
\begin{aligned}
& \omega \circ f^{*}=f^{\#} \circ \omega, \\
& \zeta \circ f^{*}=f^{*} \circ \zeta, \\
& f^{*} \circ \mathrm{a}=\mathrm{a} \circ f^{\#} .
\end{aligned}
$$

Proof. Let $\alpha \in \widehat{\mathrm{CH}}^{p}\left(Y, \mathcal{C}_{Y}\right)$ be an arithmetic cycle. By the moving lemma over $\mathbb{R}$, there is a representative $\left(z, \mathfrak{g}_{z}\right)$ of $\alpha$ such that $f^{-1}\left(\operatorname{supp} z_{\mathbb{R}}\right)$ has codimension $p$ in $X_{\mathbb{R}}$. We define $f^{*}(\alpha)$ as the class of $f^{*}\left(z, \mathfrak{g}_{z}\right)$. It remains to show that $f^{*}(\alpha)$ does not depend on the choice of the representative $\left(z, \mathfrak{g}_{z}\right)$. In order to do this, let $\left(z^{\prime}, \mathfrak{g}_{z^{\prime}}\right)$ be another representative, i.e.

$$
\left(z, \mathfrak{g}_{z}\right)-\left(z^{\prime}, \mathfrak{g}_{z^{\prime}}\right)=\widehat{\operatorname{div}}(h)
$$

for some $K_{1}$-chain $h$. Writing $U=Y \backslash\left(\operatorname{supp} z \cup \operatorname{supp} z^{\prime}\right)$, the $K_{1}$-chain $h$ determines an element $[h] \in \mathrm{CH}^{p, p-1}(U)$. By Theorem 1.39 and Proposition 1.56, there exists an element $f^{*}[h] \in \mathrm{CH}^{p, p-1}\left(f^{-1}(U)\right)$ satisfying

$$
\begin{aligned}
\operatorname{div}\left(f^{*}[h]\right) & =f^{*}(\operatorname{div}(h)) \in \mathrm{CH}_{\tau(p)}^{p}(X), \\
\operatorname{cl}\left(f^{*}[h]\right) & =f^{\#}(\operatorname{cl}(h)) \in H_{\mathcal{C}_{X}}^{2 p}\left(f^{-1}(U), p\right) .
\end{aligned}
$$

These two equations then imply

$$
f^{*}\left(z, \mathfrak{g}_{z}\right)-f^{*}\left(z^{\prime}, \mathfrak{g}_{z^{\prime}}\right)=\widehat{\operatorname{div}}\left(f^{*}(h)\right),
$$

which proves the first statement. The statements (ii), (iii) are now shown easily.

\section{Multiplicativity of inverse images}

Since the pull-back of cycles is compatible with the intersection product of cycles by Theorem 1.38, we obtain the following result using Proposition 3.62.

Theorem 4.31. Let $\left(f, f_{\mathcal{C}}^{\#}\right):\left(X, \mathcal{C}_{X}\right) \rightarrow\left(Y, \mathcal{C}_{Y}\right),\left(f, f_{\mathcal{C}^{\prime}}^{\#}\right):\left(X, \mathcal{C}_{X}^{\prime}\right) \rightarrow\left(Y, \mathcal{C}_{Y}^{\prime}\right),\left(f, f_{\mathcal{C}^{\prime \prime}}^{\#}\right):$ $\left(X, \mathcal{C}_{X}^{\prime \prime}\right) \rightarrow\left(Y, \mathcal{C}_{Y}^{\prime \prime}\right)$ be morphisms of $\mathcal{G}$-arithmetic varieties, and let $\mathcal{C}_{X} \otimes \mathcal{C}_{X}^{\prime} \stackrel{\bullet}{\longrightarrow} \mathcal{C}_{X}^{\prime \prime}$, 
$\mathcal{C}_{Y} \otimes \mathcal{C}_{Y}^{\prime} \stackrel{\bullet_{Y}}{\longrightarrow} \mathcal{C}_{Y}^{\prime \prime}$ be $\mathcal{G}$-pairings which are compatible with the $f$-morphisms $f_{\mathcal{C}}^{\#}, f_{\mathcal{C}^{\prime}}^{\#}$ and $f_{\mathcal{C}^{\prime \prime}}^{\#}$. Then, the diagram

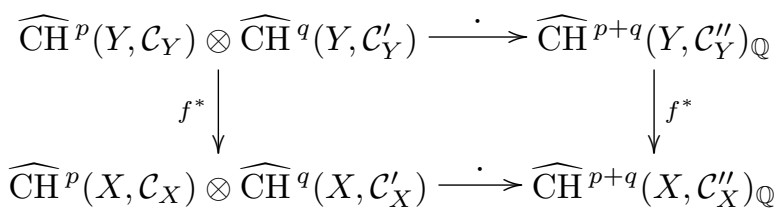

is commutative. If $p=1$, or $q=1$ the same result holds true without tensoring with $\mathbb{Q}$.

\section{Change of $\mathcal{G}$-complex}

The results for inverse images apply in particular to the case of a change of complexes on $X$, i.e. when there is a morphism of $\mathcal{G}$-arithmetic varieties

$$
f=\left(\mathrm{id}, f^{\#}\right):\left(X, \mathcal{C}^{\prime}\right) \rightarrow(X, \mathcal{C}) .
$$

Moreover, a direct consequence of Proposition 3.64 is the following result.

Proposition 4.32. Let $f=\left(\mathrm{id}, f^{\#}\right):\left(X, \mathcal{C}^{\prime}\right) \rightarrow(X, \mathcal{C})$ be a morphism of $\mathcal{G}$-arithmetic varieties such that the morphism

$$
f^{\#}: \mathcal{C}(X, p) \rightarrow \mathcal{C}^{\prime}(X, p)
$$

is an isomorphism for any integer $p$, and such that the morphism

$$
f^{\#}: \mathcal{C}(U, p) \rightarrow \mathcal{C}^{\prime}(U, p)
$$

is a quasi-isomorphism for any open subset $U \subseteq X$ and any integer $p$. Then, the induced morphism

$$
f^{*}: \widehat{\mathrm{CH}}^{p}(X, \mathcal{C}) \rightarrow \widehat{\mathrm{CH}}^{p}\left(X, \mathcal{C}^{\prime}\right)
$$

is an isomorphism for any integer $p$.

\subsection{Proper push-forward}

Let $f:\left(X, \mathcal{C}_{X}\right) \rightarrow\left(Y, \mathcal{C}_{Y}\right)$ be a covariant morphism or, more generally, a covariant pseudo-morphism of $\mathcal{G}$-arithmetic varieties of relative dimension $d$. In this section we will construct a push-forward morphism for arithmetic Chow groups.

\section{Push-forward}

Let $\left(z, \mathfrak{g}_{z}\right) \in \widehat{\mathrm{Z}}^{p}\left(X, \mathcal{C}_{X}\right)$ be a $p$-codimensional arithmetic cycle. By Theorem 1.40, there is a well-defined cycle $f_{*}(z) \in \mathrm{Z}^{p-d}(Y)$. Moreover, there is a well-defined Green object $f_{\#}\left(\mathfrak{g}_{z}\right)$ for the class of $f_{*}\left(z_{\mathbb{R}}\right)$ (see Definition 3.66 ). We will write

$$
f_{*}\left(z, \mathfrak{g}_{z}\right)=\left(f_{*}(z), f_{\#}\left(\mathfrak{g}_{z}\right)\right) .
$$


Theorem 4.34. Let $f:\left(X, \mathcal{C}_{X}\right) \rightarrow\left(Y, \mathcal{C}_{Y}\right)$ be a covariant morphism or a covariant pseudo-morphism of $\mathcal{G}$-arithmetic varieties of relative dimension $d$. Then, the following statements hold.

(i) There is a well-defined morphism of graded groups

$$
f_{*}: \widehat{\mathrm{CH}}^{p}\left(X, \mathcal{C}_{X}\right) \rightarrow \widehat{\mathrm{CH}}^{p-d}\left(Y, \mathcal{C}_{Y}\right)
$$

induced by equation (4.32) for arithmetic cycles $\left(z, \mathfrak{g}_{z}\right) \in \widehat{\mathrm{Z}}^{p}\left(X, \mathcal{C}_{X}\right)$.

(ii) If $g:\left(Y, \mathcal{C}_{Y}\right) \rightarrow\left(Z, \mathcal{C}_{Z}\right)$ is another covariant morphism or covariant pseudomorphism of $\mathcal{G}$-arithmetic varieties, then the equality $(g \circ f)_{*}=g_{*} \circ f_{*}$ holds.

(iii) The push-forward of arithmetic cycles satisfies the following relations:

$$
\begin{aligned}
\omega \circ f_{*} & =f_{\#} \circ \omega, \\
\zeta \circ f_{*} & =f_{*} \circ \zeta, \\
f_{*} \circ \mathrm{a} & =\mathrm{a} \circ f_{\#} .
\end{aligned}
$$

Proof. We will treat here only the case of a covariant morphism of $\mathcal{G}$-arithmetic varieties; the case of a covariant pseudo-morphism can be treated analogously.

The only thing that remains to be shown is the compatibility with rational equivalence. For this purpose, let $h \in R_{p}^{p-1}(X)$ be a $K_{1}$-chain. By Theorem 1.40 and Proposition 1.57, there is a well-defined $K_{1}$-chain $f_{*}(h) \in R_{p-d}^{p-d-1}(Y)$ satisfying

$$
\begin{aligned}
\operatorname{div}\left(f_{*}(h)\right) & =f_{*}(\operatorname{div}(h)), \\
\operatorname{cl}_{\mathcal{G}}\left(f_{*}(h)\right) & =f_{\#}\left(\operatorname{cl}_{\mathcal{G}}(h)\right) .
\end{aligned}
$$

By the definition of a covariant morphism of $\mathcal{G}$-arithmetic varieties, equation (4.35) implies that $\operatorname{cl}_{\mathcal{C}_{Y}}\left(f_{*}(h)\right)=f_{\#}\left(\operatorname{cl}_{\mathcal{C}_{X}}(h)\right)$. Therefore, we have $\mathfrak{g}\left(f_{*}(h)\right)=f_{\#}(\mathfrak{g}(h))$, and consequently

$$
f_{*}(\widehat{\operatorname{div}}(h))=\widehat{\operatorname{div}}\left(f_{*}(h)\right)
$$

\section{Projection formula}

The next result is a direct consequence of the projection formula for algebraic cycles and Proposition 3.78 .

Proposition 4.37. Let $\left(f, f^{\#}\right):\left(X, \mathcal{C}_{X}\right) \rightarrow\left(Y, \mathcal{C}_{Y}\right)$ be a morphism of $\mathcal{G}$-arithmetic varieties, and let

$$
\begin{aligned}
& \left(f, f_{\#}^{\prime}\right):\left(X, \mathcal{C}_{X}^{\prime}\right) \rightarrow\left(Y, \mathcal{C}_{Y}^{\prime}\right), \\
& \left(f, f_{\#}^{\prime \prime}\right):\left(X, \mathcal{C}_{X}^{\prime \prime}\right) \rightarrow\left(Y, \mathcal{C}_{Y}^{\prime \prime}\right)
\end{aligned}
$$


be covariant morphisms of $\mathcal{G}$-arithmetic varieties. Let

$$
\mathcal{C}_{X} \otimes \mathcal{C}_{X}^{\prime} \stackrel{\bullet X}{\longrightarrow} \mathcal{C}_{X}^{\prime \prime}, \quad \mathcal{C}_{Y} \otimes \mathcal{C}_{Y}^{\prime} \stackrel{\bullet}{\longrightarrow} \mathcal{C}_{Y}^{\prime \prime}
$$

be $\mathcal{G}$-pairings such that $\left(f^{\#}, f_{\#}^{\prime}, f_{\#}^{\prime \prime}, \bullet_{X}, \bullet_{Y}\right)$ is a projection five-tuple. Then, the projection formula

$$
f_{*}^{\prime \prime}\left(f^{*}(\alpha) \cdot \beta\right)=\alpha \cdot f_{*}^{\prime}(\beta)
$$

holds for all $\alpha \in \widehat{\mathrm{CH}}^{p}\left(Y, \mathcal{C}_{Y}\right)$ and $\beta \in \widehat{\mathrm{CH}}^{q}\left(X, \mathcal{C}_{X}^{\prime}\right)$.

Remark 4.38. An analogous result also holds when $f_{\#}^{\prime}$ and $f_{\#}^{\prime \prime}$ are covariant pseudomorphisms. We leave it to the reader to make this result explicit.

\section{Deligne-Beilinson cohomology as a Gillet cohomology}

A particular example for a Gillet cohomology is Deligne-Beilinson cohomology for real and complex algebraic varieties. In this section we recall the definition and some facts on Deligne-Beilinson cohomology and homology which we will use in the sequel. The main references for this section are $[\mathbf{4}],[\mathbf{2 7}]$ and $[\mathbf{4 8}]$. We start by recalling some properties of general Deligne-Beilinson cohomology; later we will shift to real Deligne-Beilinson cohomology. We will show that it satisfies most of the properties of a Gillet cohomology. Moreover, we will construct an explicit Gillet complex for real Deligne-Beilinson cohomology. Note that the results of this section are well known and we include them for the convenience of the reader.

Notation 5.1. By a complex algebraic manifold we mean the analytic variety associated to a smooth separated scheme of finite type over $\mathbb{C}$.

\subsection{Review of Deligne-Beilinson cohomology}

\section{The definition of Deligne-Beilinson cohomology}

Let $X$ be a complex algebraic manifold. Let $\Lambda$ be a subring of $\mathbb{R}$, and set $\Lambda(p)=$ $(2 \pi \mathrm{i})^{p} \Lambda \subseteq \mathbb{C}$. We will denote the corresponding constant sheaves on $X$ also by $\Lambda$, respectively $\Lambda(p)$.

Let us choose a smooth compactification $\bar{X}$ of $X$ with $D=\bar{X} \backslash X$ a normal crossing divisor, and denote by $j: X \rightarrow \bar{X}$ the natural inclusion. Let $\Omega_{X}^{*}$ be the sheaf of holomorphic differential forms on $X$, and let $\Omega_{\frac{*}{X}}^{*}(\log D)$ be the sheaf of holomorphic differential forms on $\bar{X}$ with logarithmic poles along $D$ (see $[\mathbf{2 3}]$ ). Let $F$ be the Hodge filtration of $\Omega_{\frac{*}{X}}^{*}(\log D)$, i.e.

$$
F^{p} \Omega_{\bar{X}}^{*}(\log D)=\bigoplus_{p^{\prime} \geqslant p} \Omega_{\frac{p^{\prime}}{X}}(\log D) .
$$

Then, the Deligne-Beilinson complex of the pair $(X, \bar{X})$ is given by the simple complex

$$
\Lambda(p)_{\mathcal{D}}=s\left(R j_{*} \Lambda(p) \oplus F^{p} \Omega_{\bar{X}}^{*}(\log D) \stackrel{u}{\rightarrow} j_{*} \Omega_{X}^{*}\right),
$$

where the morphism $u$ is defined by $u(a, f)=-a+f$. 
Definition 5.2. The Deligne-Beilinson cohomology groups are the hypercohomology groups of the sheaf $\Lambda(p)_{\mathcal{D}}$, i.e.

$$
H_{\mathcal{D}}^{*}(X, \Lambda(p))=\mathbb{H}^{*}\left(\bar{X}, \Lambda(p)_{\mathcal{D}}\right) .
$$

It can be shown that these groups are independent of the compactification $\bar{X}$ of $X$. The Deligne-Beilinson cohomology groups can also be constructed as the hypercohomology groups of a complex of sheaves of graded abelian groups in the Zariski topology (see $[\mathbf{2 7}, \S 5]$ for details). We will denote the corresponding sheaf in the Zariski topology by $\Lambda(*)_{\mathcal{D} \text {,Zar }}$; we will not need the precise definition of this sheaf for general $\Lambda$. In contrast, in Definition 5.32, we will give a concrete construction in the case $\Lambda=\mathbb{R}$.

\section{An exact sequence}

The definition of Deligne-Beilinson cohomology as the cohomology of a simple complex associated to a morphism of complexes implies the existence of some exact sequences. One of these exact sequences relates Deligne-Beilinson cohomology to the usual cohomology and shows that the integral Deligne-Beilinson cohomology is an extension of the group of Hodge cycles by an intermediate Jacobian. We recall the construction of this exact sequence. There is a natural map

$$
\Lambda(p)_{\mathcal{D}} \rightarrow R j_{*} \Lambda(p)
$$

given by $(a, f, \omega) \mapsto a$. The kernel of this morphism is the simple

$$
s\left(F^{p} \Omega_{\bar{X}}^{*}(\log D) \rightarrow j_{*} \Omega_{X}^{*}\right) .
$$

Since the differential is strict with respect to the Hodge filtration (see $[\mathbf{2 3}]$ ), the cohomology of this simple complex is given by $H^{*}(X, \mathbb{C}) / F^{p} H^{*}(X, \mathbb{C})$. Therefore, there is a long exact sequence

$$
\cdots \rightarrow H^{n-1}(X, \mathbb{C}) / F^{p} H^{n-1}(X, \mathbb{C}) \rightarrow H_{\mathcal{D}}^{n}(X, \Lambda(p)) \rightarrow H^{n}(X, \Lambda(p)) \rightarrow \cdots
$$

\section{The product in Deligne-Beilinson cohomology}

Beilinson has introduced a product in this cohomology. More specifically, he has introduced a family of pairings indexed by the interval $[0,1]$. For $0 \leqslant \alpha \leqslant 1$, the pairing $\cup_{\alpha}$ is given by

$$
\begin{aligned}
(a, f, \omega) \cup_{\alpha} & \left(a^{\prime}, f^{\prime}, \omega^{\prime}\right) \\
& =\left(a a^{\prime}, f \wedge f^{\prime}, \alpha\left(\omega \wedge a^{\prime}+(-1)^{n} f \wedge \omega^{\prime}\right)+(1-\alpha)\left(\omega \wedge f^{\prime}+(-1)^{n} a \wedge \omega^{\prime}\right)\right),
\end{aligned}
$$

where $(a, f, \omega) \in \Lambda(p)_{\mathcal{D}}^{n}$ and $\left(a^{\prime}, f^{\prime}, \omega^{\prime}\right) \in \Lambda(q)_{\mathcal{D}}^{m}$. All these pairings turn out to be homotopically equivalent. If $\alpha=0,1$, the pairing is associative and, if $\alpha=1 / 2$, the pairing is graded commutative. Therefore, we obtain a well-defined commutative and associative pairing in Deligne-Beilinson cohomology. 
Deligne-Beilinson cohomology and the Picard group

The Axiom $1.2(\mathrm{xi})$ of [34] states for Deligne-Beilinson cohomology that there is a natural transformation of functors

$$
\operatorname{Pic}(\cdot) \rightarrow H_{\mathcal{D}}^{2}(\cdot, \Lambda(1))
$$

This natural transformation is realized by a morphism in the derived category of the category of graded complexes of Zariski sheaves of abelian groups

$$
\mathrm{c}_{1}: \mathcal{O}_{\text {alg }}^{\times}[-1] \rightarrow \Lambda(1)_{\mathcal{D}, \text { Zar }}
$$

we refer to $[\mathbf{2 7}, \S 5]$ for the precise construction. Here we only sketch a slightly different version in the case $\Lambda=\mathbb{Z}$; the other cases then follow by functoriality. Since, for $p \geqslant 1$ and $n \leqslant 0$, we have $H_{\mathcal{D}}^{n}(X, \mathbb{Z}(p))=0$, we can assume that the complex of sheaves $\mathbb{Z}(1)_{\mathcal{D}}$, Zar starts in degree 1 . Therefore, $\mathrm{c}_{1}$ is determined by a functorial isomorphism

$$
\mathrm{c}_{1}: H^{0}\left(X, \mathcal{O}_{\text {alg }}^{\times}\right) \rightarrow H_{\mathcal{D}}^{1}(X, \mathbb{Z}(1))
$$

which can be described as follows. Using the quasi-isomorphism

$$
\mathbb{Z}(1) \rightarrow s\left(\mathcal{O}_{X} \stackrel{\exp }{\longrightarrow} \mathcal{O}_{X}^{\times}[-1]\right),
$$

one sees that $\mathbb{Z}(1)_{\mathcal{D}}$ is quasi-isomorphic to the complex

$$
s\left(R j_{*} \mathcal{O}_{X}^{\times} \oplus F^{1} \Omega_{\bar{X}}^{*}(\log D) \stackrel{u^{\prime}}{\rightarrow} R j_{*}\left(\Omega_{X}^{*} / \mathcal{O}_{X}\right)\right),
$$

where $u^{\prime}(a, f)=-\mathrm{d} \log a+f$. Hence, the group $H_{\mathcal{D}}^{1}(X, \mathbb{Z}(1))$ is isomorphic to

$$
\left\{(f, \omega) \in H^{0}\left(X, \mathcal{O}_{X}^{\times}\right) \oplus H^{0}\left(\bar{X}, \Omega_{\bar{X}}^{1}(\log D)\right) \mid \mathrm{d} \log f=\omega\right\} .
$$

By the GAGA principle the morphism $H^{0}\left(X, \mathcal{O}_{\text {alg }}^{\times}\right) \rightarrow H_{\mathcal{D}}^{1}(X, \mathbb{Z}(1))$ given by $\omega \mapsto$ $(\omega, \mathrm{d} \log \omega)$ is an isomorphism.

\subsection{Review of Deligne algebras}

In this section we will recall some definitions and results from [17] about Dolbeault algebras and their associated Deligne algebras. The interest in Deligne algebras is explained by the fact that they are very simple objects which compute real DeligneBeilinson cohomology.

\section{Dolbeault complexes}

Definition 5.7. A Dolbeault complex $A=\left(A_{\mathbb{R}}^{*}, \mathrm{~d}_{A}\right)$ is a graded complex of real vector spaces, which is bounded from below and equipped with a bigrading on $A_{\mathbb{C}}=A_{\mathbb{R}} \otimes_{\mathbb{R}} \mathbb{C}$, i.e.

$$
A_{\mathbb{C}}^{n}=\bigoplus_{p+q=n} A^{p, q},
$$

satisfying the following properties. 
(i) The differential $\mathrm{d}_{A}$ can be decomposed as the sum $\mathrm{d}_{A}=\partial+\bar{\partial}$ of operators $\partial$ of type $(1,0)$, respectively $\bar{\partial}$ of type $(0,1)$.

(ii) It satisfies the symmetry property $\overline{A^{p, q}}=A^{q, p}$, where the bar denotes complex conjugation.

Notation 5.8. Given a Dolbeault complex $A=\left(A_{\mathbb{R}}^{*}, \mathrm{~d}_{A}\right)$, we will use the following notation. The Hodge filtration $F$ of $A$ is the decreasing filtration of $A_{\mathbb{C}}$ given by

$$
F^{p} A^{n}=F^{p} A_{\mathbb{C}}^{n}=\bigoplus_{p^{\prime} \geqslant p} A^{p^{\prime}, n-p^{\prime}}
$$

The filtration $\bar{F}$ of $A$ is the complex conjugate of $F$, i.e.

$$
\bar{F}^{p} A^{n}=\bar{F}^{p} A_{\mathbb{C}}^{n}=\overline{F^{p} A_{\mathbb{C}}^{n}}
$$

For an element $x \in A_{\mathbb{C}}$, we write $x^{i, j}$ for its component in $A^{i, j}$. For $k, k^{\prime} \geqslant 0$, we define an operator $F^{k, k^{\prime}}: A_{\mathbb{C}} \rightarrow A_{\mathbb{C}}$ by the rule

$$
F^{k, k^{\prime}}(x):=\sum_{l \geqslant k, l^{\prime} \geqslant k^{\prime}} x^{l, l^{\prime}}
$$

We note that the operator $F^{k, k^{\prime}}$ is the projection of $A_{\mathbb{C}}^{*}$ onto the subspace $F^{k} A^{*} \cap \bar{F}^{k^{\prime}} A^{*}$. We will write $F^{k}=F^{k,-\infty}$.

We denote by $A_{\mathbb{R}}^{n}(p)$ the subgroup $(2 \pi \mathrm{i})^{p} \cdot A_{\mathbb{R}}^{n} \subseteq A_{\mathbb{C}}^{n}$, and we define the operator

$$
\pi_{p}: A_{\mathbb{C}} \rightarrow A_{\mathbb{R}}(p)
$$

by setting $\pi_{p}(x):=\frac{1}{2}\left(x+(-1)^{p} \bar{x}\right)$.

The Deligne complex

To any Dolbeault complex we can associate a Deligne complex.

Definition 5.9. Let $A$ be a Dolbeault complex. We denote by $A^{*}(p)_{\mathcal{D}}$ the complex $s\left(A_{\mathbb{R}}(p) \oplus F^{p} A \stackrel{u}{\rightarrow} A_{\mathbb{C}}\right)$, where $u(a, f)=-a+f$.

Definition 5.10. The Deligne complex $\left(\mathcal{D}^{*}(A, *), \mathrm{d}_{\mathcal{D}}\right)$ associated to a Dolbeault complex $A$ is the graded complex given by

$$
\mathcal{D}^{n}(A, p)= \begin{cases}A_{\mathbb{R}}^{n-1}(p-1) \cap F^{n-p, n-p} A_{\mathbb{C}}^{n-1}, & \text { if } n \leqslant 2 p-1, \\ A_{\mathbb{R}}^{n}(p) \cap F^{p, p} A_{\mathbb{C}}^{n}, & \text { if } n \geqslant 2 p,\end{cases}
$$

with differential given by $\left(x \in \mathcal{D}^{n}(A, p)\right)$

$$
\mathrm{d}_{\mathcal{D}} x= \begin{cases}-F^{n-p+1, n-p+1} \mathrm{~d}_{A} x, & \text { if } n<2 p-1, \\ -2 \partial \bar{\partial} x, & \text { if } n=2 p-1, \\ \mathrm{~d}_{A} x, & \text { if } n \geqslant 2 p .\end{cases}
$$




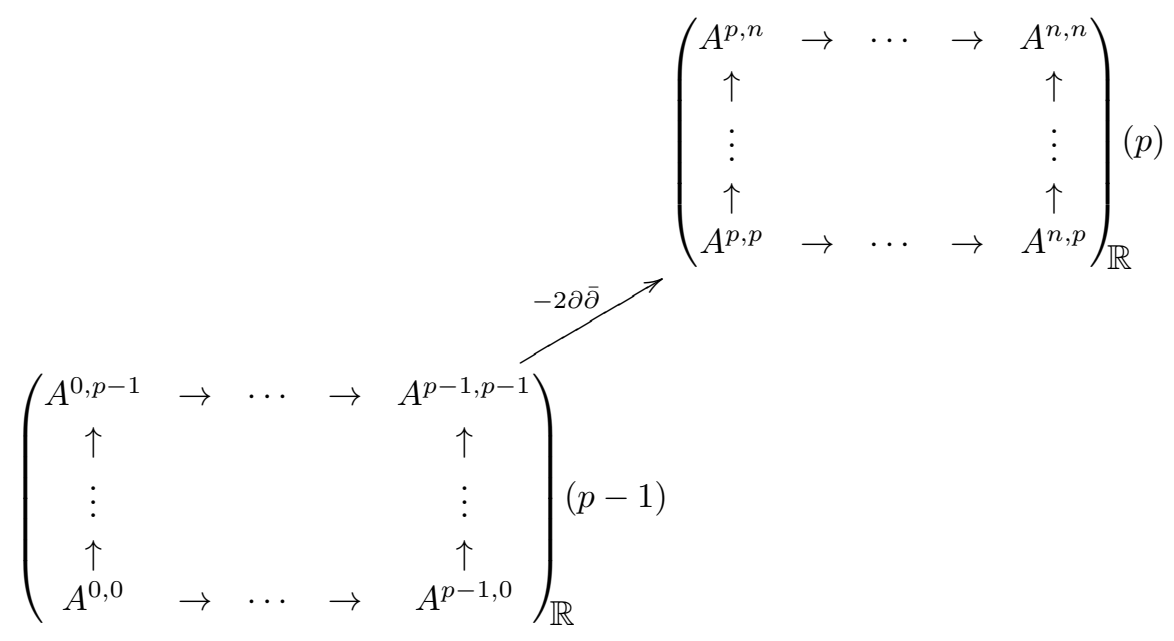

Figure 1. $\mathcal{D}(A, p)$.

For instance, if $A$ is a Dolbeault complex satisfying $A^{p, q}=0$ for $p<0, q<0, p>n$, or $q>n$, then the complex $\mathcal{D}(A, 0)$ agrees with the real complex $A_{\mathbb{R}}^{*}$; for $p>0$, we have represented $\mathcal{D}(A, p)$ in Figure 1 below, where the lower left square is shifted by one; this means in particular that $A^{0,0}$ sits in degree 1 and $A^{p-1, p-1}$ sits in degree $2 p-1$.

Remark 5.11. It is clear from the definition that the functor $\mathcal{D}(\cdot, p)$ is exact for all $p$.

The main property of the Deligne complex is expressed by the following proposition; for a proof see [17].

Proposition 5.12. The complexes $A^{*}(p)_{\mathcal{D}}$ and $\mathcal{D}^{*}(A, p)$ are homotopically equivalent. The homotopy equivalences $\psi: A^{n}(p)_{\mathcal{D}} \rightarrow \mathcal{D}^{n}(A, p)$ and $\varphi: \mathcal{D}^{n}(A, p) \rightarrow A^{n}(p)_{\mathcal{D}}$ are given by

$$
\psi(a, f, \omega)= \begin{cases}\pi(\omega), & \text { if } n \leqslant 2 p-1, \\ F^{p, p} a+2 \pi_{p}\left(\partial \omega^{p-1, n-p+1}\right), & \text { if } n \geqslant 2 p,\end{cases}
$$

where $\pi(\omega)=\pi_{p-1}\left(F^{n-p, n-p} \omega\right)$, i.e. $\pi$ is the projection of $A_{\mathbb{C}}$ over the cokernel of $u$, and

$$
\varphi(x)= \begin{cases}\left(\partial x^{p-1, n-p}-\bar{\partial} x^{n-p, p-1}, 2 \partial x^{p-1, n-p}, x\right), & \text { if } n \leqslant 2 p-1 \\ (x, x, 0), & \text { if } n \geqslant 2 p\end{cases}
$$

Moreover, $\psi \circ \varphi=\mathrm{id}$, and $\varphi \circ \psi-\mathrm{id}=\mathrm{d} h+h \mathrm{~d}$, where $h: A^{n}(p)_{\mathcal{D}} \rightarrow A^{n-1}(p)_{\mathcal{D}}$ is given by

$$
h(a, f, \omega)= \begin{cases}\left(\pi_{p}\left(\bar{F}^{p} \omega+\bar{F}^{n-p} \omega\right),-2 F^{p}\left(\pi_{p-1} \omega\right), 0\right), & \text { if } n \leqslant 2 p-1, \\ \left(2 \pi_{p}\left(\bar{F}^{n-p} \omega\right),-F^{p, p} \omega-2 F^{n-p}\left(\pi_{p-1} \omega\right), 0\right), & \text { if } n \geqslant 2 p .\end{cases}
$$




\section{Example}

Let $X$ be a complex projective manifold, and $\mathcal{E}_{X}^{*}$ the sheaf of smooth, complex differential forms on $X$. Then, the complex of global sections $E_{X}^{*}$ of $\mathcal{E}_{X}^{*}$ has a natural structure of Dolbeault complex. The cohomology of the complex $\mathcal{D}^{*}\left(E_{X}, p\right)$ is naturally isomorphic to the real Deligne-Beilinson cohomology $H_{\mathcal{D}}^{*}(X, \mathbb{R}(p))$ of $X$ (see $[\mathbf{2 5}]$ ).

\section{The product in the Deligne complex}

The multiplicative structure of a Dolbeault algebra induces a product in the Deligne complex which is graded commutative and associative up to homotopy.

Definition 5.13. A Dolbeault algebra $A=\left(A_{\mathbb{R}}^{*}, \mathrm{~d}_{A}, \wedge\right)$ is a Dolbeault complex equipped with an associative and graded commutative product

$$
\wedge: A_{\mathbb{R}}^{*} \times A_{\mathbb{R}}^{*} \rightarrow A_{\mathbb{R}}^{*}
$$

such that the induced multiplication on $A_{\mathbb{C}}^{*}$ is compatible with the bigrading, i.e.

$$
A^{p, q} \wedge A^{p^{\prime}, q^{\prime}} \subseteq A^{p+p^{\prime}, q+q^{\prime}} .
$$

Definition 5.14. Let $A$ be a Dolbeault algebra. The Deligne algebra associated to $A$ is the Deligne complex $\mathcal{D}^{*}(A, *)$ together with the graded commutative product - : $\mathcal{D}^{n}(A, p) \times \mathcal{D}^{m}(A, q) \rightarrow \mathcal{D}^{n+m}(A, p+q)$ given by

$$
x \bullet y= \begin{cases}(-1)^{n} r_{p}(x) \wedge y+x \wedge r_{q}(y), & \text { if } n<2 p, m<2 q, \\ F^{l-r, l-r}(x \wedge y), & \text { if } n<2 p, m \geqslant 2 q, l<2 r, \\ F^{r, r}\left(r_{p}(x) \wedge y\right)+2 \pi_{r}\left(\partial(x \wedge y)^{r-1, l-r}\right), & \text { if } n<2 p, m \geqslant 2 q, l \geqslant 2 r, \\ x \wedge y, & \text { if } n \geqslant 2 p, m \geqslant 2 q,\end{cases}
$$

where we have written $l=n+m, r=p+q$, and $r_{p}(x)=2 \pi_{p}\left(F^{p} \mathrm{~d}_{A} x\right)$.

For a proof of the next result we refer to $[\mathbf{1 7}]$.

Proposition 5.15. Let $\mathcal{D}^{*}(A, *)$ be the Deligne algebra associated to a Dolbeault algebra $A$. Then, we have the following statements.

(i) The product $\bullet$ is associative up to a natural homotopy $h_{a}$.

(ii) For $x \in \mathcal{D}^{2 p}(A, p), y \in \mathcal{D}^{2 q}(A, q), z \in \mathcal{D}^{2 r}(A, r)$, we have

$$
h_{a}(x \otimes y \otimes z)=0,
$$

i.e. the product $\bullet$ is pseudo-associative in the sense of Definition 3.41; in particular, the direct sum $\bigoplus_{n \in \mathbb{Z}} \mathcal{D}^{2 n}(A, n)$ is an associative subalgebra.

(iii) There is a natural morphism of graded complexes $r_{p}: \mathcal{D}^{*}(A, p) \rightarrow A^{*}(p)$ given by

$$
r_{p}(x)= \begin{cases}\partial\left(x^{p-1, n-p}\right)-\bar{\partial}\left(x^{n-p, p-1}\right), & \text { if } n \leqslant 2 p-1 \\ x, & \text { if } n \geqslant 2 p\end{cases}
$$


here $x \in \mathcal{D}^{n}(A, p)$. This morphism is multiplicative up to homotopy and induces a morphism of graded algebras

$$
H^{*}(\mathcal{D}(A, p)) \rightarrow H^{*}(A(p)) .
$$

Remark 5.16. In his thesis [32], Fulea has introduced a new product structure for the Deligne algebra $\mathcal{D}^{*}(A, *)$, which is graded commutative and associative. Using this product structure one may simplify some of the constructions of the present paper; in particular, one can avoid the use of pseudo-associativity.

Specific degrees

In the sequel we will be interested in some specific degrees where we can give simpler formulae. Namely, we consider

$$
\begin{aligned}
\mathcal{D}^{2 p}(A, p) & =A_{\mathbb{R}}^{2 p}(p) \cap A^{p, p}, \\
\mathcal{D}^{2 p-1}(A, p) & =A_{\mathbb{R}}^{2 p-2}(p-1) \cap A^{p-1, p-1}, \\
\mathcal{D}^{2 p-2}(A, p) & =A_{\mathbb{R}}^{2 p-3}(p-1) \cap\left(A^{p-2, p-1} \oplus A^{p-1, p-2}\right) .
\end{aligned}
$$

The corresponding differentials are given by

$$
\begin{aligned}
\mathrm{d}_{\mathcal{D}} x & =\mathrm{d}_{A} x, & & \text { if } x \in \mathcal{D}^{2 p}(A, p), \\
\mathrm{d}_{\mathcal{D}} x & =-2 \partial \bar{\partial} x, & & \text { if } x \in \mathcal{D}^{2 p-1}(A, p), \\
\mathrm{d}_{\mathcal{D}}(x, y) & =-\partial x-\bar{\partial} y, & & \text { if }(x, y) \in \mathcal{D}^{2 p-2}(A, p) .
\end{aligned}
$$

Moreover, the product is given as follows: for $x \in \mathcal{D}^{2 p}(A, p), y \in \mathcal{D}^{2 q}(A, q)$ or $y \in$ $\mathcal{D}^{2 q-1}(A, q)$, we have

$$
x \bullet y=x \wedge y,
$$

and for $x \in \mathcal{D}^{2 p-1}(A, p), y \in \mathcal{D}^{2 q-1}(A, q)$, we have

$$
x \bullet y=-\partial x \wedge y+\bar{\partial} x \wedge y+x \wedge \partial y-x \wedge \bar{\partial} y .
$$

\section{Dolbeault modules}

Once we have defined Dolbeault algebras we may introduce the concept of Dolbeault modules. The main example is the space of currents over a variety as a module over the space of differential forms.

Definition 5.17. Let $A$ be a Dolbeault algebra and $M$ a Dolbeault complex. We say that $M$ is a Dolbeault module over $A$, if $M$ is a differential graded module satisfying

$$
A^{p, q} M^{p^{\prime}, q^{\prime}} \subseteq M^{p+p^{\prime}, q+q^{\prime}} .
$$

The following proposition is straightforward.

Proposition 5.18. Let $A$ be a Dolbeault algebra and $M$ a Dolbeault module over $A$. Then, $\mathcal{D}^{*}(M, *)$ is a differential graded module over $\mathcal{D}^{*}(A, *)$. Moreover, the action is pseudo-associative. 
Deligne complexes and Deligne-Beilinson cohomology

The main interest in Deligne complexes is expressed by the following theorem which is proven in [17] in a particular case, although the proof is valid in general. It is a consequence of Proposition 5.12.

Theorem 5.19. Let $X$ be a complex algebraic manifold, $\bar{X}$ a smooth compactification of $X$ with $D=\bar{X} \backslash X$ a normal crossing divisor, and denote by $j: X \rightarrow \bar{X}$ the natural inclusion. Let $\mathcal{A}^{*}$ be a sheaf of Dolbeault algebras over $\bar{X}^{\text {an }}$ such that, for every $n, p$, the sheaves $\mathcal{A}^{n}$ and $F^{p} \mathcal{A}^{n}$ are acyclic, $\mathcal{A}^{*}$ is a multiplicative resolution of $R j_{*} \mathbb{R}$, and $\left(\mathcal{A}_{\mathbb{C}}^{*}, F\right)$ is a multiplicative filtered resolution of $\left(\Omega_{\bar{X}}^{*}(\log D), F\right)$. Putting $A^{*}=\Gamma\left(\bar{X}, \mathcal{A}^{*}\right)$, we have a natural isomorphism of graded algebras

$$
H_{\mathcal{D}}^{*}(X, \mathbb{R}(p)) \cong H^{*}(\mathcal{D}(A, p))
$$

Moreover, the morphism $r_{p}$ of Proposition 5.15 (iii) induces the natural morphism of graded algebras

$$
H_{\mathcal{D}}^{*}(X, \mathbb{R}(p)) \rightarrow H^{*}(X, \mathbb{R}(p))
$$

\subsection{A Gillet complex: the Deligne algebra $\mathcal{D}_{\log }$}

Smooth differential forms with logarithmic singularities

Let $W$ be a complex algebraic manifold and $D$ a normal crossing divisor in $W$. We put $X=W \backslash D$, and denote by $j: X \rightarrow W$ the natural inclusion. We recall that $\mathcal{E}_{W}^{*}$ denotes the sheaf of smooth, complex differential forms on $W$.

Definition 5.20. The complex of sheaves $\mathcal{E}_{W}^{*}(\log D)$ of differential forms with logarithmic singularities along $D$ is the $\mathcal{E}_{W}^{*}$-subalgebra of $j_{*} \mathcal{E}_{X}^{*}$, which is locally generated by the sections

$$
\log z_{i} \bar{z}_{i}, \frac{\mathrm{d} z_{i}}{z_{i}}, \frac{\mathrm{d} \bar{z}_{i}}{\bar{z}_{i}} \quad \text { for } i=1, \ldots, m
$$

where $z_{1} \cdots z_{m}=0$ is a local equation for $D$ (see $[\mathbf{1 5}]$ ).

Notation 5.21. In the sequel we will adhere to the following convention. Sheaves in the analytic topology will usually be denoted by script letters, whereas the group of global sections will be denoted by the corresponding roman letters. For instance, we will write

$$
\begin{aligned}
E_{W}^{*} & =\Gamma\left(W, \mathcal{E}_{W}^{*}\right), \\
E_{W}^{*}(\log D) & =\Gamma\left(W, \mathcal{E}_{W}^{*}(\log D)\right), \\
E_{W, \mathbb{R}}^{*}(\log D) & =\Gamma\left(W, \mathcal{E}_{W, \mathbb{R}}^{*}(\log D)\right) .
\end{aligned}
$$

With this notation, the complex $E_{W}(\log D)=\left(E_{W, \mathbb{R}}^{*}(\log D)\right.$, d) is a Dolbeault algebra. 
Suppose now that $W$ is proper. Then, there are multiplicative isomorphisms in the derived category of abelian sheaves over $W$ :

$$
\begin{aligned}
R j_{*} \mathbb{R}(p) & \rightarrow \mathcal{E}_{W, \mathbb{R}}^{*}(\log D)(p), \\
j_{*} \Omega_{X}^{*} & \rightarrow \mathcal{E}_{W}^{*}(\log D), \\
F^{p} \Omega_{W}^{*}(\log D) & \rightarrow F^{p} \mathcal{E}_{W}^{*}(\log D) .
\end{aligned}
$$

We therefore obtain the following theorem from Theorem 5.19.

Theorem 5.22. There is a natural multiplicative isomorphism

$$
H_{\mathcal{D}}^{*}(X, \mathbb{R}(p)) \rightarrow H^{*}\left(\mathcal{D}\left(E_{W}(\log D), p\right)\right) .
$$

\section{Logarithmic singularities at infinity}

We want to obtain a description of Deligne-Beilinson cohomology which is independent of a given compactification. Given a complex algebraic manifold $X$, let $I$ be the category of all smooth compactifications of $X$ with a normal crossing divisor as its complement. This means that an element $\left(\bar{X}_{\alpha}, j_{\alpha}\right)$ of $I$ consists of a proper complex algebraic manifold $\bar{X}_{\alpha}$ together with an immersion $j_{\alpha}: X \rightarrow \bar{X}_{\alpha}$ such that $D_{\alpha}=\bar{X}_{\alpha} \backslash j_{\alpha}(X)$ is a normal crossing divisor. The morphisms of $I$ are the maps $f: \bar{X}_{\alpha} \rightarrow \bar{X}_{\beta}$ satisfying $f \circ j_{\alpha}=j_{\beta}$. It can be shown that the opposite category $I^{o}$ is directed (see $[\mathbf{2 3}]$ ).

We put

$$
E_{\log }^{*}(X)^{\circ}=\underset{\alpha \in I^{\circ}}{\lim _{\alpha}} E_{\bar{X}_{\alpha}}^{*}\left(\log D_{\alpha}\right) ;
$$

it is clear that the vector spaces $E_{\log }^{*}(X)^{\circ}$ form a complex of presheaves $E_{\log }^{*}{ }^{\circ}$ in the Zariski topology.*

The corresponding real subcomplex $E_{\log }(X)^{\circ}=\left(E_{\log , \mathbb{R}}^{*}(X)^{\circ}, \mathrm{d}\right)$ is a presheaf of Dolbeault algebras. Moreover, by the results of [15], if $f: \bar{X}_{\alpha} \rightarrow \bar{X}_{\beta}$ is a morphism of $I$, the induced morphism

$$
f^{*}:\left(E_{\bar{X}_{\beta}}^{*}\left(\log D_{\beta}\right), F\right) \rightarrow\left(E_{\bar{X}_{\alpha}}^{*}\left(\log D_{\alpha}\right), F\right)
$$

is a real filtered quasi-isomorphism. Since $I^{o}$ is directed, all the induced morphisms

$$
\left(E_{\bar{X}_{\alpha}}^{*}\left(\log D_{\alpha}\right), F\right) \rightarrow\left(E_{\log }^{*}(X)^{\circ}, F\right)
$$

are also real filtered quasi-isomorphisms.

We will denote by $E_{\log }^{*}$ the complex of sheaves in the Zariski topology associated to the complex of presheaves $E_{\log }^{*}$.

Definition 5.24. The complex of differential forms with logarithmic singularities along infinity is defined by

$$
E_{\log }^{*}(X)=\Gamma\left(X, E_{\log }^{*}\right)
$$

it is a subalgebra of $E_{X}^{*}=\Gamma\left(X, \mathcal{E}_{X}^{*}\right)$. We denote the corresponding real subcomplex by $E_{\log , \mathbb{R}}^{*}(X)$.

\footnotetext{
* Added in proof: in fact, $E_{\log }^{*}{ }^{\circ}$ is a sheaf (see [19, Theorem 3.6]).
} 
Remark 5.25. The natural map $E_{\log }^{*}(X)^{\circ} \rightarrow E_{\log }^{*}(X)$ is injective, but not surjective, in general.

Pseudo-flasque complexes of presheaves

In order to understand the relationship between the cohomology of the complexes $E_{\log }^{*}(X)$ and $E_{\log }^{*}(X)^{\circ}$, we introduce pseudo-flasque complexes of presheaves.

Definition 5.26. Let $X$ be a scheme and $\mathcal{F}^{*}$ a complex of presheaves in the Zariski topology of $X$. Then, $\mathcal{F}^{*}$ is called pseudo-flasque, if $\mathcal{F}^{*}(\emptyset)=0$ and if, for every pair of open subsets $U, V$, the natural map

$$
\mathcal{F}(U \cup V) \rightarrow s(\mathcal{F}(U) \oplus \mathcal{F}(V) \rightarrow \mathcal{F}(U \cap V))
$$

is a quasi-isomorphism.

For instance, any complex of totally acyclic sheaves is a pseudo-flasque complex of presheaves.

The basic property of pseudo-flasque complexes of presheaves is the analogue of Proposition 1.12 .

Proposition 5.27. Let $\mathcal{F}$ be a pseudo-flasque complex of presheaves, and let $\mathcal{F}^{\prime}$ be the associated complex of sheaves. Then, for any scheme $X$, we have

$$
\mathbb{H}^{n}\left(X, \mathcal{F}^{\prime}\right)=H^{n}(\Gamma(X, \mathcal{F}))
$$

Remark 5.28. It is possible to generalize the notion of $\mathcal{G}$-complexes using pseudo-flasque complexes of presheaves. All the theory developed in $\S \S 3$ and 4 can be generalized with minor modifications.

Proposition 5.29. The natural morphism

$$
\left(E_{\log }^{*}(X)^{\circ}, F\right) \rightarrow\left(E_{\log }^{*}(X), F\right)
$$

is a filtered quasi-isomorphism.

Proof. By the filtered quasi-isomorphism (5.22), the filtered complex $\left(E_{\log }^{*}(X)^{\circ}, F\right)$ computes the cohomology of $X$ with complex coefficients with its Hodge filtration. Since the Mayer-Vietoris sequence for the cohomology of $X$ with complex coefficients is an exact sequence of mixed Hodge structures, it induces Mayer-Vietoris sequences for the graded pieces with respect to the Hodge filtration. Therefore, the complexes of presheaves $E_{\log }^{p, *}(X)^{\circ}$ are pseudo-flasque. By a partition of unity argument (see the discussion before Lemma 6.11$)$ it is easy to see that the sheaves $E_{\log }^{p, q}(X)$ are totally acyclic. Hence, the result follows from Proposition 5.27. 
A Deligne complex with logarithmic singularities

Definition 5.30. Let $X$ be a complex algebraic manifold. For any integer $p$, we put

$$
\mathcal{D}_{\log }^{*}(X, p)=\mathcal{D}^{*}\left(E_{\log }(X), p\right)
$$

By the exactness of the functor $\mathcal{D}(\cdot, p)$, Theorem 5.22 and Proposition 5.29, we obtain the following corollary.

Corollary 5.31. There is a natural multiplicative isomorphism

$$
H_{\mathcal{D}}^{*}(X, \mathbb{R}(p)) \rightarrow H^{*}\left(\mathcal{D}_{\log }(X, p)\right)
$$

\section{A Gillet complex for real Deligne-Beilinson cohomology}

We denote by $C$ the site of regular schemes in $\mathrm{ZAR}(\operatorname{Spec}(\mathbb{C}))$. In particular, we recall that all schemes in $C$ are separated and of finite type over $\operatorname{Spec}(\mathbb{C})$. For a scheme $X$ in $C$, the set of complex points $X(\mathbb{C})$ is a complex algebraic manifold.

Definition 5.32. For any integers $n, p$, let $\mathcal{D}_{\log }^{n}(p)$ denote the presheaf (in fact, a sheaf) over $C$, which assigns to $X$ the group

$$
\mathcal{D}_{\log }^{n}(X, p)=\mathcal{D}_{\log }^{n}(X(\mathbb{C}), p)=\mathcal{D}^{n}\left(E_{\log }(X(\mathbb{C})), p\right) .
$$

For any scheme $X$ in $C$, we will denote the induced presheaf of graded complexes of real vector spaces on $X$ by $\mathcal{D}_{\log , X}=\mathcal{D}_{\log , X}^{*}(*)$.

Proposition 5.33. For any integers $n, p$, the presheaf $\mathcal{D}_{\log , X}^{n}(p)$ is a totally acyclic sheaf on $X$.

Proof. This follows from the facts that the functor $\mathcal{D}(\cdot, p)$ is exact and that the sheaves $E_{\log }^{p, q}$ are totally acyclic.

Theorem 5.34. The graded complex of sheaves of abelian groups $\mathcal{D}_{\log }$ is a Gillet complex for regular schemes over $\mathbb{C}$, which computes real Deligne-Beilinson cohomology. Moreover, the pair $\left(\mathcal{D}_{\log }, \bullet\right)$ is a graded commutative and pseudo-associative algebra for real Deligne-Beilinson cohomology.

Proof. Since $\mathcal{D}_{\log }$ is a totally acyclic sheaf in the Zariski topology, the hypercohomology agrees with the cohomology of the complex of global sections. By Theorem 5.19, the complex of global sections computes real Deligne-Beilinson cohomology. Since [48] shows that real Deligne-Beilinson cohomology satisfies the Gillet axioms, the complex $\mathcal{D}_{\log }$ is a Gillet complex. Finally, Proposition 5.15 and Theorem 5.19 imply the claimed multiplicative properties. 


\subsection{Deligne-Beilinson homology of proper smooth varieties}

The homology theory associated to a Gillet cohomology determines direct images and classes for algebraic cycles. For this reason we have to discuss the construction and basic properties of Deligne-Beilinson homology. In general, Deligne-Beilinson homology is defined by means of currents and smooth singular chains (for details, see [48]). But since we are only interested in real Deligne-Beilinson homology, we do not need to use singular chains. Apart from some minor changes, we will follow [48]. In particular, unless stated otherwise, we will follow the conventions therein.

\section{Currents}

Let $X$ be a complex algebraic manifold. The sheaf ${ }^{\prime} \mathcal{E}_{X}^{n}$ of currents of degree $n$ on $X$ is defined as follows. For any open subset $U$ of $X$, the group ${ }^{\prime} \mathcal{E}_{X}^{n}(U)$ is the topological dual of the group of sections with compact support $\Gamma_{c}\left(U, \mathcal{E}_{X}^{-n}\right)$. The differential

$$
\mathrm{d}:{ }^{\prime} \mathcal{E}_{X}^{n} \rightarrow{ }^{\prime} \mathcal{E}_{X}^{n+1}
$$

is defined by

$$
\mathrm{d} T(\varphi)=(-1)^{n} T(\mathrm{~d} \varphi)
$$

here $T$ is a current and $\varphi$ a corresponding test form.

The real structure and the bigrading of $\mathcal{E}_{X}^{*}$ induce a real structure and a bigrading of ${ }^{\prime} \mathcal{E}_{X}^{*}$. Furthermore, there is a pairing

$$
\mathcal{E}_{X}^{n} \otimes{ }^{\prime} \mathcal{E}_{X}^{m} \rightarrow{ }^{\prime} \mathcal{E}_{X}^{n+m}, \quad \omega \otimes T \mapsto \omega \wedge T,
$$

where the current $\omega \wedge T$ is defined by

$$
(\omega \wedge T)(\eta)=T(\eta \wedge \omega)
$$

This pairing, the real structure and the bigrading equip ${ }^{\prime} \mathcal{E}_{X}^{*}$ with the structure of a Dolbeault module ${ }^{\prime} \mathcal{E}_{X}=\left({ }^{\prime} \mathcal{E}_{X, \mathbb{R}}^{*}, \mathrm{~d}\right)$ over the Dolbeault algebra $\mathcal{E}_{X}=\left(\mathcal{E}_{X, \mathbb{R}}^{*}, \mathrm{~d}\right)$.

Deligne-Beilinson homology

Definition 5.35. Let $X$ be a proper complex algebraic manifold. Then, the real DeligneBeilinson homology groups of $X$ are defined by

$$
{ }^{\prime} H_{\mathcal{D}}^{*}(X, \mathbb{R}(p))=H^{*}\left(\mathcal{D}\left({ }^{\prime} E_{X}, p\right)\right),
$$

where, as fixed in Notation 5.21 , we have written ${ }^{\prime} E_{X}^{*}=\Gamma\left(X,{ }^{\prime} \mathcal{E}_{X}^{*}\right)$. We will also write

$$
H_{n}^{\mathcal{D}}(X, \mathbb{R}(p))={ }^{\prime} H_{\mathcal{D}}^{-n}(X, \mathbb{R}(-p)) .
$$

Remark 5.36. The proof that the above definition of the real Deligne-Beilinson homology groups agrees with Beilinson's definition as given in $[\mathbf{4}]$ or $[\mathbf{4 8}]$, is completely analogous to the proof of Theorem 5.19 given in [17]. 
Equidimensional varieties

Definition 5.37. Let $X$ be an equidimensional complex algebraic manifold of dimension $d$. Then, we put

$$
\mathcal{D}_{X}^{*}={ }^{\prime} \mathcal{E}_{X}^{*}[-2 d](-d),
$$

and, according to Notation 5.21,

$$
D_{X}^{*}=\Gamma\left(X, \mathcal{D}_{X}^{*}\right)
$$

In particular, we note

$$
\begin{aligned}
\mathcal{D}_{X}^{p, q} & ={ }^{\prime} \mathcal{E}_{X}^{p-d, q-d}, \\
\mathcal{D}_{X, \mathbb{R}}^{*}(p) & =(2 \pi \mathrm{i})^{p-d} \cdot{ }^{\prime} \mathcal{E}_{X, \mathbb{R}}^{*} ;
\end{aligned}
$$

therefore, the subcomplex of real currents $\mathcal{D}_{X, \mathbb{R}}^{n}(U, p)$ is the topological dual of $\Gamma_{c}\left(U, \mathcal{E}_{X, \mathbb{R}}^{2 d-n}(p-d)\right)$.

The current associated to a differential form

Let $X$ be an equidimensional complex algebraic manifold of dimension $d$. Then, there is a natural morphism of sheaves

$$
\mathcal{E}_{X}^{*} \rightarrow \mathcal{D}_{X}^{*}
$$

given by $\omega \mapsto[\omega]$, where the current $[\omega]$ is defined by

$$
[\omega](\eta)=\frac{1}{(2 \pi \mathrm{i})^{d}} \int_{X} \eta \wedge \omega,
$$

for a corresponding test form $\eta$ with compact support. This morphism is compatible with the structure of the Dolbeault module $\mathcal{D}_{X}=\left(\mathcal{D}_{X, \mathbb{R}}^{*}, \mathrm{~d}\right)$ over the Dolbeault algebra $\mathcal{E}_{X}=\left(\mathcal{E}_{X, \mathbb{R}}^{*}, \mathrm{~d}\right)$.

We will use the same notation and normalization for any locally integrable differential form $\omega$.

\section{Poincaré duality}

The local version of Poincaré duality is the following theorem. For a proof we refer, for example, to [39, p. 384].

Theorem 5.39 (local Poincaré duality). Let $X$ be an equidimensional complex algebraic manifold. Then, the morphism $\left(\mathcal{E}_{X}^{*}, F\right) \rightarrow\left(\mathcal{D}_{X}^{*}, F\right)$ is a filtered quasi-isomorphism, which is compatible with the underlying real structures.

From the local version of Poincaré duality, one derives the following corollary.

Corollary 5.40 (Poincaré duality). Let $X$ be a proper equidimensional complex algebraic manifold of dimension $d$. Then, there is a natural isomorphism

$$
H_{\mathcal{D}}^{n}(X, \mathbb{R}(p)) \rightarrow{ }^{\prime} H_{\mathcal{D}}^{n-2 d}(X, \mathbb{R}(p-d))=H_{2 d-n}^{\mathcal{D}}(X, \mathbb{R}(d-p)) .
$$




\section{Direct images}

Let $f: X \rightarrow Y$ be a proper morphism between complex algebraic manifolds. Then, we define a morphism of sheaves

$$
f_{!}: f_{*}{ }^{\prime} \mathcal{E}_{X}^{*} \rightarrow{ }^{\prime} \mathcal{E}_{Y}^{*}
$$

by setting $\left(f_{!} T\right)(\eta)=T\left(f^{*} \eta\right)$ for a test form $\eta$. When $X$ and $Y$ are proper, this morphism induces a morphism

$$
f_{!}:{ }^{\prime} H_{\mathcal{D}}^{*}(X, p) \rightarrow{ }^{\prime} H_{\mathcal{D}}^{*}(Y, p) .
$$

If furthermore $X$ and $Y$ are equidimensional, and $f$ has relative dimension $e$, the morphism $f_{\text {! sends }} \mathcal{D}_{X, \mathbb{R}}^{n}(p)$ to $\mathcal{D}_{Y, \mathbb{R}}^{n-2 e}(p-e)$. Therefore, by Poincaré duality, for each $n, p$, we obtain an induced morphism

$$
f_{!}: H_{\mathcal{D}}^{n}(X, \mathbb{R}(p)) \rightarrow H_{\mathcal{D}}^{n-2 e}(Y, \mathbb{R}(p-e)) .
$$

If $f$ is smooth and $\omega$ is a differential form on $X$, we will write

$$
f ! \omega=\frac{1}{(2 \pi \mathrm{i})^{e}} \int_{f} \omega
$$

for the integration of $\omega$ along the fibre. It turns out that the definition of a current associated to a differential form and of push-forwards are compatible, i.e.

$$
f_{!}[\omega]=\left[f_{!} \omega\right] .
$$

\section{The fundamental class}

Let $X$ be a proper equidimensional complex algebraic manifold of dimension $d$. Then, the fundamental class of Axiom 1.2 (iv) of [34] in

$$
H_{\mathcal{D}}^{0}(X, \mathbb{R}(0)) \cong H_{2 d}^{\mathcal{D}}(X, \mathbb{R}(d))={ }^{\prime} H_{\mathcal{D}}^{-2 d}(X, \mathbb{R}(-d))
$$

is determined by the constant function 1 . Therefore, the fundamental class can be represented by the current

$$
\delta_{X}=[1]
$$

The class of a cycle

Definition 5.41. Let $X$ be a complex algebraic manifold, and $Y$ an $e$-dimensional irreducible subvariety of $X$. Let $\tilde{Y}$ be a resolution of singularities of $Y$, and $\imath: \widetilde{Y} \rightarrow X$ the induced map. Then, the current integration along $Y$, denoted by $\delta_{Y}$, is defined by

$$
\delta_{Y}=\imath ! \delta_{\widetilde{Y}} \in \mathcal{D}^{-2 e}\left({ }^{\prime} E_{X},-e\right)={ }^{\prime} E_{X, \mathbb{R}}^{-2 e}(-e) \cap{ }^{\prime} E_{X}^{-e,-e} .
$$

Therefore, it satisfies

$$
\delta_{Y}(\eta)=\frac{1}{(2 \pi \mathrm{i})^{e}} \int_{\widetilde{Y}} \imath^{*} \eta .
$$

If $X$ is equidimensional of dimension $d$ and $p=d-e$, we obtain by the convention on the real structure of $\mathcal{D}_{X}^{*}$ that $\delta_{Y}$ is an element of $\mathcal{D}^{2 p}\left(D_{X}, p\right)=D_{X, \mathbb{R}}^{2 p}(p) \cap D_{X}^{p, p}$. By linearity, we define $\delta_{y}$ for any algebraic cycle $y$. 
Since the current $\delta_{Y}$ is closed, the following definition makes sense.

Definition 5.42. Let $X$ be a proper complex algebraic manifold, and $y$ an $e$-dimensional algebraic cycle of $X$. The homology class of $y$, denoted by $\operatorname{cl}_{\mathcal{D}}(y)$ or $\operatorname{simply}$ by $\operatorname{cl}(y)$, is the class in ${ }^{\prime} H_{\mathcal{D}}^{-2 e}(X, \mathbb{R}(-e))$ represented by $\delta_{y}$. If $X$ is equidimensional of dimension $d$ and $p=d-e$, the (cohomology) class of $y$ in $H_{\mathcal{D}}^{2 p}(X, \mathbb{R}(p))$, also denoted by $\operatorname{cl}_{\mathcal{D}}(y)$ or $\operatorname{cl}(y)$, is defined by Poincaré duality.

\subsection{Deligne-Beilinson homology of arbitrary varieties}

Up to now, we have discussed Deligne-Beilinson homology only for proper complex algebraic manifolds. Therefore, we still have to treat the case of non-proper complex algebraic manifolds. Moreover, even if a Gillet cohomology is only defined for smooth varieties, the homology should also be defined for singular varieties. In this section we will discuss Deligne-Beilinson homology for general varieties. By abuse of notation, we will denote in this section a complex variety and its associated analytic space by the same letter. This will not cause confusion, since we consider only sheaves in the analytic topology in this section.

Note that the complexes of currents which we will use are slightly different from the complexes used in $[48]$.

\section{Currents on a subvariety}

Let $X$ be a complex algebraic manifold, $Y$ a closed subvariety of $X$, and $j: Y \rightarrow X$ the natural inclusion. We put

$$
\Sigma_{Y} \mathcal{E}_{X}^{*}=\left\{\omega \in \mathcal{E}_{X}^{*} \mid j^{*} \omega=0\right\}
$$

Definition 5.43. The sheaf of currents on $Y$ is defined by

$$
{ }^{\prime} \mathcal{E}_{Y}^{*}=\left\{T \in{ }^{\prime} \mathcal{E}_{X}^{*} \mid T(\omega)=0 \forall \omega \in \Sigma_{Y} \mathcal{E}_{X}^{*}\right\} .
$$

Furthermore, we put

$$
{ }^{\prime} \mathcal{E}_{X / Y}^{*}={ }^{\prime} \mathcal{E}_{X}^{*} /{ }^{\prime} \mathcal{E}_{Y}^{*}
$$

The sheaf ' $\mathcal{E}_{Y}^{*}$ was introduced by Bloom and Herrera in [8]. If $Y$ is smooth, it agrees with the usual definition. The sheaf ${ }^{\prime} \mathcal{E}_{X / Y}^{n}$ is the sheaf of distributions for $\Sigma_{Y} \mathcal{E}_{X}^{-n}$. We point out that when $Y$ is a normal crossing divisor, the complex of sheaves ' $\mathcal{E}_{X / Y}^{*}$ does not agree with the complex denoted by ' $\Omega_{X^{\infty}}^{*}\langle Y\rangle$ in $[\mathbf{4 8}]$ because this last complex is not defined over $\mathbb{R}$ (see also [51]). Note, however, that, as a consequence of Theorem 5.44, both complexes are filtered quasi-isomorphic.

If $X$ is equidimensional of dimension $d$, we put as before

$$
\begin{aligned}
\mathcal{D}_{X / Y}^{*} & ={ }^{\prime} \mathcal{E}_{X / Y}^{*}[-2 d](-d), \\
\overline{\mathcal{D}}_{Y}^{*} & ={ }^{\prime} \mathcal{E}_{Y}^{*}[-2 d](-d) .
\end{aligned}
$$


We observe that the grading for the complex $\overline{\mathcal{D}}_{Y}^{*}$ is relative to the dimension of $X$ and not to the dimension of $Y$. Therefore, if $Y$ is smooth, the complex $\overline{\mathcal{D}}_{Y}^{*}$ does not agree with the complex $\mathcal{D}_{Y}^{*}$.

The complex ' $\mathcal{E}_{X / Y}^{*}$ defines a Dolbeault complex. If $X$ is equidimensional of dimension $d$, the complex $\mathcal{D}_{X / Y}^{*}$ also defines a Dolbeault complex. In particular, both complexes have a well-defined Hodge filtration.

\section{Normal crossing divisors}

Theorem 5.44. Let $X$ be an equidimensional complex algebraic manifold of dimension $d$, and $Y$ a normal crossing divisor in $X$. Then, there is a well-defined morphism of complexes

$$
\mathcal{E}_{X}^{*}(\log Y) \rightarrow \mathcal{D}_{X / Y}^{*}, \quad \omega \mapsto[\omega]
$$

satisfying

$$
[\omega](\eta)=\frac{1}{(2 \pi \mathrm{i})^{d}} \int_{U} \eta \wedge \omega
$$

where $U$ is an open subset of $X, \omega \in \Gamma\left(U, \mathcal{E}_{X}^{*}(\log Y)\right)$, and $\eta \in \Gamma_{c}\left(U, \Sigma_{Y} \mathcal{E}_{X}^{*}\right)$. Moreover, this morphism is a quasi-isomorphism with respect to the Hodge filtration.

Proof. The fact that we have a well-defined morphism of complexes is proven in $[\mathbf{1 6}, 3.3]$.

All the ingredients for the proof that the morphism in question is a filtered quasiisomorphism are contained in [31]. We put

$$
\begin{aligned}
& \mathcal{E}_{X}^{*}(\operatorname{hol} \log Y)=\Omega_{X}^{*}(\log Y) \otimes_{\Omega_{X}^{*}} \mathcal{E}_{X}^{*}, \\
& \mathcal{Q}_{X}^{*}(\operatorname{hol} \log Y)=\mathcal{E}_{X}^{*}(\operatorname{hol} \log Y) / \mathcal{E}_{X}^{*} .
\end{aligned}
$$

Since these complexes are not defined over $\mathbb{R}$, they do not define Dolbeault complexes. Nevertheless, we can define the Hodge filtration in the usual way.

By [15], the natural inclusion

$$
\mathcal{E}_{X}^{*}(\operatorname{hol} \log Y) \rightarrow \mathcal{E}_{X}^{*}(\log Y)
$$

is a filtered quasi-isomorphism with respect to the Hodge filtration. Thus, it is enough to show that the composition

$$
\mathcal{E}_{X}^{*}(\operatorname{hol} \log Y) \rightarrow \mathcal{D}_{X / Y}^{*}
$$

is a filtered quasi-isomorphism.

Following [31] and [43], we define a map PV : $\mathcal{E}_{X}^{*}(\operatorname{hol} \log Y) \rightarrow \mathcal{D}_{X}^{*}$ by setting

$$
\mathrm{PV}(\omega)(\eta)=\frac{1}{(2 \pi \mathrm{i})^{d}} \int_{U} \eta \wedge \omega
$$

where $U$ is an open subset of $X, \omega \in \Gamma\left(U, \mathcal{E}_{X}^{*}(\operatorname{hol} \log Y)\right)$, and $\eta \in \Gamma_{c}\left(U, \mathcal{E}_{X}^{*}\right)$. We note that the map PV is not a morphism of complexes. 
Starting with the commutative diagram

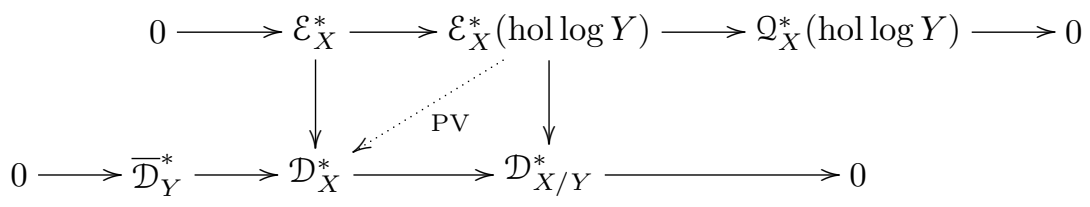

we define a morphism of complexes

$$
\operatorname{Res}: \mathcal{Q}_{X}^{*}(\operatorname{hol} \log Y)[-1] \rightarrow \overline{\mathcal{D}}_{Y}^{*}
$$

by

$$
\operatorname{Res}(\omega)=\mathrm{dPV}(\bar{\omega})-\operatorname{PV}(\mathrm{d} \bar{\omega})
$$

where $\bar{\omega}$ is any representative of $\omega$ in $\mathcal{E}_{X}^{*}(\operatorname{hol} \log Y)[-1]$. It is easy to see that Res is well defined and its image lies in $\overline{\mathcal{D}}_{Y}^{*}$. By the local description of Res given in $[\mathbf{3 1}, 3.6]$, it is a homogeneous morphism of bidegree $(0,1)$. Thus, it is compatible with the Hodge filtration, and we obtain a map of distinguished exact triangles

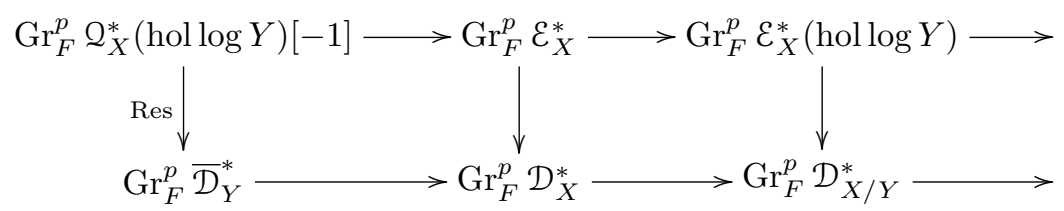

From this we conclude that, if two of the above vertical maps are quasi-isomorphisms, so is the third. By Theorem 5.39, the middle vertical arrow is a quasi-isomorphism. By means of an auxiliary complex $\mathcal{K}_{X}^{*}\langle Y\rangle$ equipped with a Hodge filtration, the following commutative triangle, where all the morphisms are filtered, is established in [31]:

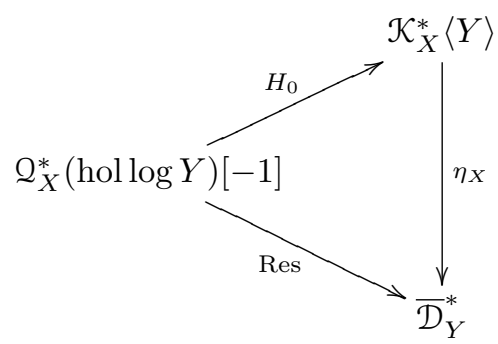

Now it is proven in $[\mathbf{3 1}]$ that all the morphisms in this diagram are quasi-isomorphisms and that $H_{0}$ is a filtered quasi-isomorphism with respect to the Hodge filtration. It is also immediate from the proof in $[\mathbf{3 1}]$ that $\eta_{X}$ is a filtered quasi-isomorphism with respect to the Hodge filtration. This proves that Res is also a filtered quasi-isomorphism with respect to the Hodge filtration from which the claim follows. 
Deligne-Beilinson homology of non-proper varieties

Definition 5.45. Let $X$ be a complex algebraic manifold, and $\bar{X}$ a smooth compactification of $X$ with $D=\bar{X} \backslash X$ a normal crossing divisor. Then, the real Deligne-Beilinson homology groups of $X$ are defined by

$$
{ }^{\prime} H_{\mathcal{D}}^{*}(X, \mathbb{R}(p))=H^{*}\left(\mathcal{D}\left({ }^{\prime} E_{\bar{X} / D}, p\right)\right)
$$

We will also write

$$
H_{n}^{\mathcal{D}}(X, \mathbb{R}(p))={ }^{\prime} H_{\mathcal{D}}^{-n}(X, \mathbb{R}(-p)) .
$$

Theorem 5.46. Real Deligne-Beilinson homology is well defined. That is, it does not depend on the choice of a compactification. Moreover, it is covariant for proper morphisms between smooth complex varieties.

Proof. The main ingredients of the proof of the first statement are, first, that given two compactifications $\bar{X}_{1}$ and $\bar{X}_{2}$ as above, there is always a third compactification $\bar{X}_{3}$ that dominates both; and, second, that given a diagram of compactifications

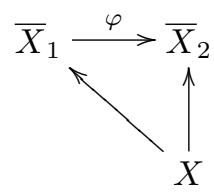

with normal crossing divisors $D_{1}$ and $D_{2}$, the morphism $\varphi$ induces a filtered quasiisomorphism

$$
\varphi_{!}: \varphi_{*}{ }^{\prime} \mathcal{E}_{\bar{X}_{1} / D_{1}}^{*} \rightarrow{ }^{\prime} \mathcal{E}_{\bar{X}_{2} / D_{2}}^{*}
$$

The main ingredient to show the covariance is that, given a proper morphism $f: X \rightarrow Y$ between smooth complex varieties, it is possible to construct a commutative diagram

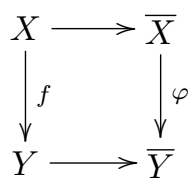

where $\bar{X}$ and $\bar{Y}$ are smooth compactifications with normal crossing divisor $D_{X}=\bar{X} \backslash X$ and $D_{Y}=\bar{Y} \backslash Y$. Then there is an induced morphism

$$
\varphi_{!}: \varphi_{*}^{\prime} \mathcal{E}_{\bar{X} / D_{X}}^{*} \rightarrow{ }^{\prime} \mathcal{E}_{\bar{Y}}^{*} / D_{Y} \cdot
$$

For more details see $[48, \S 1]$.

Poincaré duality for non-proper varieties

A direct consequence of Theorem 5.44 is the following corollary. 
Corollary 5.48 (Poincaré duality). Let $X$ be an equidimensional complex algebraic manifold of dimension $d$. Then, there is a natural isomorphism

$$
H_{\mathcal{D}}^{n}(X, \mathbb{R}(p)) \rightarrow{ }^{\prime} H_{\mathcal{D}}^{n-2 d}(X, \mathbb{R}(p-d))=H_{2 d-n}^{\mathcal{D}}(X, \mathbb{R}(d-p)) .
$$

Corollary 5.49. The definition of Deligne-Beilinson homology given here agrees with the definition given in $[\mathbf{4}]$ and $[\mathbf{4 8}]$.

\section{Direct images}

Let $f: X \rightarrow Y$ be a proper equidimensional morphism between complex algebraic manifolds, of relative dimension $e$. Then, using Poincaré duality and the covariance of Deligne-Beilinson homology, for each $n, p$, we obtain an induced morphism

$$
f_{!}: H_{\mathcal{D}}^{n}(X, \mathbb{R}(p)) \rightarrow H_{\mathcal{D}}^{n-2 e}(Y, \mathbb{R}(p-e)) .
$$

Deligne-Beilinson homology of arbitrary varieties

In order to define Deligne-Beilinson homology for a possibly singular variety, we will use simplicial resolutions. For details on simplicial resolutions and cohomological descent the reader is referred to $[\mathbf{2 4}]$ or $[\mathbf{2}]$.

Let $X$ be a variety over $\mathbb{C}$. Then, there is a smooth simplicial scheme $X$. with an augmentation $\pi: X . \rightarrow X$, which is a proper hypercovering, and hence satisfies cohomological descent. Furthermore, there is a proper smooth simplicial scheme $\bar{X}$. together with an open immersion $X . \rightarrow \bar{X}$. such that the complement $D .=\bar{X}$. \X. is a normal crossing divisor. By the covariance of currents, we obtain a simplicial graded complex $\mathcal{D}^{*}\left({ }^{\prime} E_{\bar{X}}, / D, p\right)$. Let ' $\mathcal{N}$ be the canonical normalization functor transforming a simplicial group into a cochain complex, i.e. this functor transforms a simplicial group into a chain complex and then reverses the signs of the grading.

Definition 5.50. The real Deligne-Beilinson homology groups of $X$ are defined by

$$
{ }^{\prime} H_{\mathcal{D}}^{*}(X, \mathbb{R}(p))=H^{*}\left(s\left({ }^{\prime} \mathcal{N} \mathcal{D}\left({ }^{\prime} E_{\bar{X}}, / D, p\right)\right)\right) .
$$

As usual, we will also write

$$
H_{n}^{\mathcal{D}}(X, \mathbb{R}(p))={ }^{\prime} H_{\mathcal{D}}^{-n}(X, \mathbb{R}(-p))
$$

For a proof of the next result see [48]. It is based on the same principles as the proof of Theorem 5.46 .

Theorem 5.51. Real Deligne-Beilinson homology is well defined. That is, it does not depend on the choice of a compactification of a proper hypercovering. Moreover, it is covariant for proper morphisms between complex varieties. 
Remark 5.52. If $Y$ is a normal crossing divisor of a proper smooth variety $W$, the proof of Theorem 5.44 shows that we do not need to use a simplicial resolution of $Y$ to compute the Deligne-Beilinson homology of $Y$. Indeed, the complex ${ }^{\prime} \mathcal{E}_{Y}^{*}$ is quasi-isomorphic to the one obtained by a simplicial resolution. Therefore, we have

$$
{ }^{\prime} H_{\mathcal{D}}^{*}(Y, \mathbb{R}(p))=H^{*}\left(\mathcal{D}\left({ }^{\prime} E_{Y}, p\right)\right) .
$$

More generally, if $Y$ is a normal crossing divisor of a smooth variety $W$, and $\bar{W}$ is a smooth compactification of $W$ such that $Z=\bar{W} \backslash W$ and $Y \cup Z$ are normal crossing divisors, we write

$$
{ }^{\prime} \mathcal{E}_{(Y \cup Z) / Z}^{*}={ }^{\prime} \mathcal{E}_{Y \cup Z}^{*} /^{\prime} \mathcal{E}_{Z}^{*} .
$$

Then, we can use the graded complex $\mathcal{D}^{*}\left({ }^{\prime} E_{(Y \cup Z) / Z}, p\right)$ to compute the real DeligneBeilinson homology of $Y$.

\section{Poincaré duality for general varieties}

We will recall how to construct the long exact sequence in homology associated to a closed subvariety, and the Poincaré duality isomorphism between homology and cohomology with support. For details on the proof we refer to [48].

Let $X$ be a variety over $\mathbb{C}$, and $Y$ a closed subvariety of $X$. Then, there is a smooth simplicial scheme $X$. with an augmentation $\pi: X . \rightarrow X$, which is a proper hypercovering, and hence satisfies cohomological descent. Furthermore, there is a proper smooth simplicial scheme $\bar{X}$. together with an open immersion $X . \rightarrow \bar{X}$. such that $D .=\bar{X}$. $\backslash X$., $Y .=\pi^{-1}(Y)$, and $Z .=D \cdot \cup Y$. are normal crossing divisors. We observe that $Y . \rightarrow Y$ also satisfies cohomological descent. For $p \in \mathbb{Z}$, we now obtain a short exact sequence

$$
\begin{aligned}
0 \rightarrow s\left({ }^{\prime} \mathcal{N D}^{*}\left({ }^{\prime} E_{Z . / D .}, p\right)\right) & \rightarrow s\left({ }^{\prime} \mathcal{N} \mathcal{D}^{*}\left({ }^{\prime} E_{\bar{X} . / D}, p\right)\right) \\
& \rightarrow s\left({ }^{\prime} \mathcal{N} \mathcal{D}^{*}\left({ }^{\prime} E_{\bar{X} . / Z}, p\right)\right) \rightarrow 0
\end{aligned}
$$

From this exact sequence we obtain a long exact sequence of homology groups

$$
\cdots \rightarrow{ }^{\prime} H_{\mathcal{D}}^{*}(Y, \mathbb{R}(p)) \rightarrow{ }^{\prime} H_{\mathcal{D}}^{*}(X, \mathbb{R}(p)) \rightarrow{ }^{\prime} H_{\mathcal{D}}^{*}(X \backslash Y, \mathbb{R}(p)) \rightarrow \cdots .
$$

Theorem 5.54 (Poincaré duality). Let $X$ be an equidimensional complex algebraic manifold of dimension $d$, and $Y$ a closed subvariety of $X$. Then, there is a natural isomorphism

$$
H_{n}^{\mathcal{D}}(Y, \mathbb{R}(p)) \rightarrow H_{\mathcal{D}, Y}^{2 d-n}(X, \mathbb{R}(d-p)) .
$$

Proof. Let $\bar{X}$ be a smooth compactification of $X$ with $D=\bar{X} \backslash X$ a normal crossing divisor. Then, we may assume that the simplicial resolution $\bar{X}$. used to construct the exact sequence (5.52) satisfies the conditions that $\bar{X}_{0}$ is a proper modification of $\bar{X}$, which is an isomorphism over the complement of the adherence of $Y$ and furthermore that $\bar{X} \rightarrow \bar{X}$ is a proper hypercovering. We define $D$., $Y$., and $Z$. as before. Then $\bar{X}_{0} \backslash Z_{0}$ can be identified with $X \backslash Y$. Therefore, the cohomology group $H_{\mathcal{D}, Y}^{*}(X, \mathbb{R}(p))$ is computed as the cohomology of the simple complex associated to the morphism of graded complexes

$$
\mathcal{D}^{*}\left(E_{\bar{X}}(\log D), p\right) \rightarrow \mathcal{D}^{*}\left(E_{\bar{X}_{0}}\left(\log Z_{0}\right), p\right) .
$$


For any variety $S$ occurring in the simplicial resolution $\bar{X}$., we put

$$
\overline{\mathcal{D}}_{S}^{*}={ }^{\prime} \mathcal{E}_{S}^{*}[-2 d](-d)
$$

i.e. all the dimensions are considered relatively to $X$. The condition of $\bar{X} . \rightarrow \bar{X}$ being a proper hypercovering implies the existence of a quasi-isomorphism

$$
s\left({ }^{\prime} \mathcal{N} \mathcal{D}^{*}\left(\bar{D}_{\bar{X} . / D}, p\right)\right) \rightarrow \mathcal{D}^{*}\left(\bar{D}_{\bar{X} / D}, p\right) .
$$

Then, there is a commutative diagram

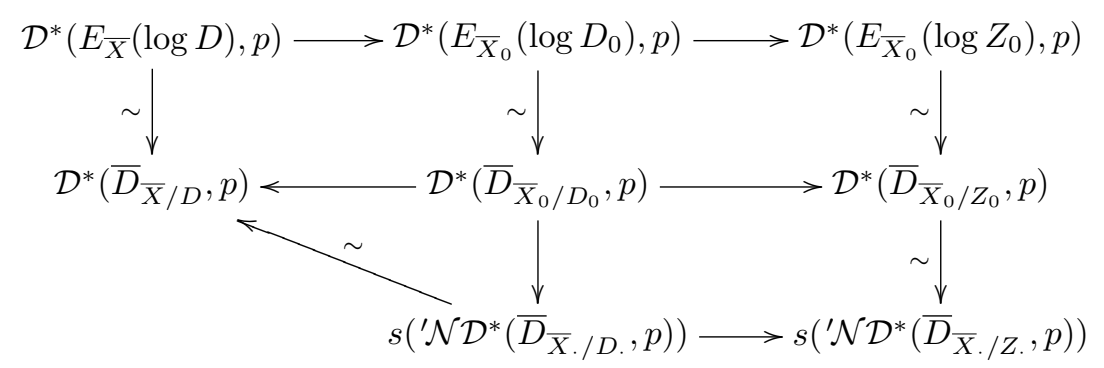

where the arrows marked with ' $\sim$ ' are quasi-isomorphisms. This shows that the cohomology groups $H_{\mathcal{D}, Y}^{*}(X, \mathbb{R}(p))$ can be computed as the cohomology of the simple complex associated to the morphism of graded complexes

$$
s\left({ }^{\prime} \mathcal{N} \mathcal{D}^{*}\left(\bar{D}_{\bar{X} . / D}, p\right)\right) \rightarrow s\left({ }^{\prime} \mathcal{N} \mathcal{D}^{*}\left(\bar{D}_{\bar{X} . / Z,}, p\right)\right)
$$

The result now follows from the exact sequence (5.52) and Poincaré duality for smooth varieties.

\section{Direct images}

We are finally able to define direct images for Deligne-Beilinson cohomology with support. Let $f: X \rightarrow Y$ be a proper equidimensional morphism between smooth complex varieties, of relative dimension $e$. Let $Z \subseteq X$ and $Z^{\prime} \subseteq Y$ be closed subsets such that $f(Z) \subseteq Z^{\prime}$. Then, for each $n, p$, there is a morphism

$$
f_{!}: H_{\mathcal{D}, Z}^{n}(X, \mathbb{R}(p)) \rightarrow H_{\mathcal{D}, Z^{\prime}}^{n-2 e}(Y, \mathbb{R}(p-e))
$$

induced by Poincaré duality and the covariance of Deligne-Beilinson homology.

There are two special cases where the direct image can be easily described in terms of differential forms with logarithmic singularities. The proof is left to the reader.

\section{Proposition 5.56.}

(i) Let $X$ be a proper smooth complex variety and let $Z \subseteq Y$ be closed subsets. Let $\widetilde{X}_{Z}$ be an embedded resolution of singularities of $Z$ with normal crossing divisor $D_{Z}$ and let $\widetilde{X}_{Y}$ be an embedded resolution of singularities of $Y$ with normal crossing divisor $D_{Y}$ that dominates $\tilde{X}_{Z}$. Then the morphism $H_{\mathcal{D}, Z}^{n}(X, \mathbb{R}(p)) \rightarrow H_{\mathcal{D}, Y}^{n}(X, \mathbb{R}(p))$ is induced by the natural morphism

$$
s\left(\mathcal{D}^{*}\left(E_{X}, p\right) \rightarrow \mathcal{D}^{*}\left(E_{\widetilde{X}_{Z}}\left(\log D_{Z}\right), p\right)\right) \rightarrow s\left(\mathcal{D}^{*}\left(E_{X}, p\right) \rightarrow \mathcal{D}^{*}\left(E_{\widetilde{X}_{Y}}\left(\log D_{Y}\right), p\right)\right) .
$$


(ii) Let $f: X \rightarrow Y$ be a proper smooth morphism between proper varieties, of relative dimension e. Let $Z \subseteq Y$ be a closed subset. Let $\widetilde{Y}$ be an embedded resolution of singularities of $Z$ with normal crossing divisor $D$. We write

$$
\widetilde{X}=\underset{Y}{\times} \underset{Y}{\times}, \quad B=X \underset{Y}{\times} \widetilde{D} .
$$

By the smoothness of $f, \widetilde{X}$ is an embedded resolution of singularities of $f^{-1}(Z)$ with normal crossing divisor $B$. Then integration along the fibre induces a morphism

$$
f_{!}: \mathcal{D}^{*}\left(E_{\widetilde{X}}(\log B), p\right) \rightarrow \mathcal{D}^{*}\left(E_{\widetilde{Y}}(\log D), p-e\right)[2 e]
$$

and the morphism $H_{\mathcal{D}, f^{-1}(Z)}^{n}(X, \mathbb{R}(p)) \rightarrow H_{\mathcal{D}, Z}^{n-2 e}(Y, \mathbb{R}(p-e))$ is induced by the morphism

$$
\begin{aligned}
f_{!}: s\left(\mathcal { D } ^ { * } ( E _ { X } , p ) \rightarrow \mathcal { D } ^ { * } \left(E_{\widetilde{X}}\right.\right. & (\log B), p)) \\
& \rightarrow s\left(\mathcal{D}^{*}\left(E_{Y}, p-e\right) \rightarrow \mathcal{D}^{*}\left(E_{\widetilde{Y}}(\log D), p-e\right)\right)[-2 e] .
\end{aligned}
$$

\subsection{Classes of cycles and line bundles}

The class of a cycle in cohomology with support

We are now in a position to describe the class of a cycle in real Deligne-Beilinson cohomology with support. To do this, let $X$ be an equidimensional complex algebraic manifold of dimension $d$. Since the class of a cycle in a quasi-projective variety is the restriction of the class of a cycle in any compactification, we may assume that $X$ is projective. Then, let $y$ be a $p$-codimensional, i.e. $(d-p)$-dimensional cycle of $X$, and $Y$ the support of $y$. Letting $Y=\bigcup_{j} Y_{j}$ be the decomposition of $Y$ into irreducible components, we have $y=\sum_{j} n_{Y_{j}} Y_{j}$ with certain multiplicities $n_{j}$. If $\widetilde{Y}$. denotes a proper hypercovering of $Y$ such that $\widetilde{Y}_{0}$ is a resolution of singularities of $Y$, then $\tilde{Y}_{0}$ is a disjoint union of irreducible components $\widetilde{Y}_{0}=\bigcup_{j} \widetilde{Y}_{0, j}$ with $\widetilde{Y}_{0, j}$ a resolution of singularities of $Y_{j}$. If $n_{y}$ denotes the locally constant function with value $n_{j}$ on the component $\widetilde{Y}_{0, j}$, then $n_{y}$ defines an element $\left[n_{y}\right]$ of

$$
\mathcal{D}^{-2 d+2 p}\left({ }^{\prime} E_{\widetilde{Y}_{0}},-d+p\right)={ }^{\prime} E_{\widetilde{Y}_{0}, \mathbb{R}}^{-2 d+2 p}(-d+p) \cap{ }^{\prime} E_{\widetilde{Y}_{0}}^{-d+p,-d+p}
$$

given by

$$
\left[n_{y}\right](\eta)=\frac{1}{(2 \pi \mathrm{i})^{d-p}} \int_{\widetilde{Y}_{0}} n_{y} \cdot \eta .
$$

The element $\left[n_{y}\right]$ is a cycle of the complex $s\left(\mathcal{D}^{*}\left({ }^{\prime} E_{\widetilde{Y}},-d+p\right)\right)$, and hence defines a cohomology class in

$$
\begin{aligned}
H_{2 d-2 p}^{\mathcal{D}}(Y, \mathbb{R}(d-p)) & ={ }^{\prime} H_{\mathcal{D}}^{-2 d+2 p}(Y, \mathbb{R}(-d+p)) \\
& =H^{-2 d+2 p}\left(s\left(\mathcal{D}\left({ }^{\prime} E_{\tilde{Y}},-d+p\right)\right)\right) .
\end{aligned}
$$

The image of this class under the Poincaré duality isomorphism

$$
H_{2 d-2 p}^{\mathcal{D}}(Y, \mathbb{R}(d-p)) \stackrel{\cong}{\longrightarrow} H_{\mathcal{D}, Y}^{2 p}(X, \mathbb{R}(p))
$$


is merely the class of the cycle $y$ in $H_{\mathcal{D}, Y}^{2 p}(X, \mathbb{R}(p))$. Observing that the real mixed Hodge structure of $H_{Y}^{2 p}(X, \mathbb{R})$ is pure of type $(p, p)$ and that $H_{Y}^{2 p-1}(X, \mathbb{R})=0$, the exact sequence (5.3) implies that the natural morphism

$$
r_{p}: H_{\mathcal{D}, Y}^{2 p}(X, \mathbb{R}(p)) \rightarrow H_{Y}^{2 p}(X, \mathbb{R}(p))
$$

is an isomorphism. Therefore, the class of $y$ in $H_{\mathcal{D}, Y}^{2 p}(X, \mathbb{R}(p))$ is determined by its image in the Betti cohomology group $H_{Y}^{2 p}(X, \mathbb{R}(p))$. Since the latter group is determined as the cohomology of the simple complex

$$
s\left(D_{X}^{*}(p) \rightarrow D_{X \backslash Y}^{*}(p)\right),
$$

the class of the cycle $y$ can be represented by the pair $\left(\delta_{y}, 0\right)$.

Differential forms representing the class of a cycle

By the above considerations we have a representative for the class of a cycle in terms of currents. We now give a criterion, when a pair of differential forms represents this class. For this let

$$
(\omega, g) \in s^{2 p}\left(\mathcal{D}_{\log }(X, p) \rightarrow \mathcal{D}_{\log }(X \backslash Y, p)\right) ;
$$

by $[\mathbf{1 6}, 3.8 .2]$ the form $g$ is locally integrable on $X$. If $U=X \backslash Y$, we write $[g]_{U}$ for the current associated to $g$ as a smooth form on $U$; we write $[g]_{X}$ for the current associated to $g$ as a locally integrable form on $X$. As usual, we put $d^{\mathrm{c}}=(4 \pi \mathrm{i})^{-1}(\partial-\bar{\partial})$.

Proposition 5.58. Let $X$ be a complex algebraic manifold, and $y$ a $p$-codimensional cycle on $X$ with support $Y$. Let $(\omega, g)$ be a cycle in

$$
s^{2 p}\left(\mathcal{D}_{\log }(X, p) \rightarrow \mathcal{D}_{\log }(X \backslash Y, p)\right) .
$$

Then, we have the following statements.

(i) The class of the cycle $(\omega, g)$ in $H_{\mathcal{D}, Y}^{2 p}(X, \mathbb{R}(p))$ is equal to the class of $y$, if and only if

$$
-2 \partial \bar{\partial}[g]_{X}=[\omega]-\delta_{y}
$$

(ii) Assume that $y=\sum_{j} n_{j} Y_{j}$ with irreducible subvarieties $Y_{j}$ and certain multiplicities $n_{j}$. Then, the cycle $(\omega, g)$ represents the class of $y$, if and only if the equality

$$
-\lim _{\varepsilon \rightarrow 0} \int_{\partial B_{\varepsilon}(Y)} \alpha \mathrm{d}^{\mathrm{c}} g=\frac{(2 \pi \mathrm{i})^{p-1}}{2} \sum_{j} n_{j} \int_{Y_{j}} \alpha
$$

holds for any differential form $\alpha$; here $B_{\varepsilon}(Y)$ is an $\varepsilon$-neighbourhood of $Y$ such that the orientation of $\partial B_{\varepsilon}(Y)$ is induced from the orientation of $B_{\varepsilon}(Y)$.

Proof. (i) First, assume that the cycle $(\omega, g)$ represents the class of $y$ in the group $H_{\mathcal{D}, Y}^{2 p}(X, \mathbb{R}(p))$. Let $\bar{X}$ be a smooth compactification of $X$, and $\bar{y}$ a cycle extending $y$ with support $\bar{Y}$. By $[\mathbf{1 6}, 4.8]$ there exists a pair $\left(\omega_{1}^{\prime}, g_{1}^{\prime}\right)$ representing the class of $\bar{y}$ in 
the simple complex $s^{2 p}\left(\mathcal{D}_{\log }(\bar{X}, p) \rightarrow \mathcal{D}_{\log }(\bar{X} \backslash \bar{Y}, p)\right)$ such that $-2 \partial \bar{\partial}\left[g_{1}^{\prime}\right]_{\bar{X}}=\left[\omega_{1}^{\prime}\right]-\delta_{\bar{y}}$. We denote by $\omega_{1}$, respectively $g_{1}$ the restriction of $\omega_{1}^{\prime}$, respectively $g_{1}^{\prime}$ to $X$. By the functoriality of the class of a cycle, the pairs $\left(\omega_{1}, g_{1}\right)$ and $(\omega, g)$ represent the same class. Therefore, there are elements $a \in \mathcal{D}_{\log }^{2 p-1}(X, p)$ and $b \in \mathcal{D}_{\log }^{2 p-2}(X \backslash Y, p)$ such that

$$
\left(\mathrm{d}_{\mathcal{D}} a, a-\mathrm{d}_{\mathcal{D}} b\right)=(\omega, g)-\left(\omega_{1}, g_{1}\right)
$$

This shows

$$
\mathrm{d}_{\mathcal{D}}[g]_{X}=\mathrm{d}_{\mathcal{D}}\left[g_{1}\right]_{X}+\mathrm{d}_{\mathcal{D}}[a]=\left[\omega_{1}\right]-\delta_{y}+[\omega]-\left[\omega_{1}\right]=[\omega]-\delta_{y}
$$

Since $\mathrm{d}_{\mathcal{D}}=-2 \partial \bar{\partial}$ in degree $2 p-1$, we find that the pair $(\omega, g)$ satisfies equation $(5.58)$.

Conversely, assume that $(\omega, g)$ satisfies equation (5.58). By $[\mathbf{1 6}, 3.8 .3]$ we know that $r_{p}\left([g]_{X}\right)=\left[r_{p}(g)\right]_{X}$, where $r_{p}$ is as in Proposition 5.15 (iii). Since we have $-2 \partial \bar{\partial}[g]_{X}=$ $\mathrm{d} r_{p}\left([g]_{X}\right)$, equation (5.58) implies

$$
\mathrm{d}\left[r_{p}(g)\right]_{X}=\left[r_{p}(\omega)\right]-\delta_{y} .
$$

Hence, we obtain

$$
\mathrm{d}\left(\left[r_{p}(g)\right]_{X}, 0\right)=\left(\left[r_{p}(\omega)\right],\left[r_{p}(g)\right]_{U}\right)-\left(\delta_{y}, 0\right) .
$$

Since the natural morphism (5.56) is an isomorphism, we therefore obtain that $(\omega, g)$ represents the class of $y$.

(ii) Let $\alpha$ be a differential form and put $n=\operatorname{deg} \alpha$. Equation (5.60) is equivalent to the equation

$$
(-1)^{n+1} \frac{1}{(2 \pi \mathrm{i})^{n}} \int_{X} \mathrm{~d} \alpha \wedge r_{p}(g)=\frac{1}{(2 \pi \mathrm{i})^{n}} \int_{X} \alpha \wedge \omega-\frac{1}{(2 \pi \mathrm{i})^{n-p}} \sum_{j} n_{j} \int_{Y_{j}} \alpha .
$$

Using the fact that $(\omega, g)$ is a cycle and that $\alpha$ has even degree, the above equation is equivalent to

$$
\int_{X} \mathrm{~d}\left(\alpha \wedge r_{p}(g)\right)=(2 \pi \mathrm{i})^{p} \sum_{j} n_{j} \int_{Y_{j}} \alpha .
$$

The result now follows from Stokes's theorem and the fact that

$$
\mathrm{d}^{\mathrm{c}} g=\frac{1}{4 \pi \mathrm{i}} r_{p}(g)
$$

as shown in Proposition 5.15 (iii).

\section{The first Chern form of a line bundle}

The morphism $c_{1}$ in (5.4) normalizes the first Chern class of a line bundle and therefore of all Chern classes of vector bundles. We recall now the usual way of how to obtain representatives for the first Chern class of a line bundle. Let $X$ be a quasi-projective complex algebraic manifold, $\bar{X}$ a smooth compactification of $X$, and $L$ a line bundle over $X$. Then, $L$ can be extended to a line bundle $\bar{L}$ over $\bar{X}$. Let $\bar{h}$ be a smooth hermitian 
metric on $\bar{L}$, and $h$ the restriction of $\bar{h}$ to $L$. If $s$ is a non-vanishing, rational section of $L$, we put $\|s\|^{2}=h(s, s), y=\operatorname{div}(s), Y=\operatorname{supp} y, U=X \backslash Y$, and

$$
\begin{aligned}
g_{s} & =-\frac{1}{2} \log \left(\|s\|^{2}\right), \\
\omega_{s} & =-2 \partial \bar{\partial} g_{s} .
\end{aligned}
$$

Moreover, we put $\bar{y}=\operatorname{div}(s)$ with $s$ viewed as a section on $\bar{X}$, and $\bar{Y}=\operatorname{supp} \bar{y}$. We call

$$
\mathrm{c}_{1}(L, h)=\omega_{s}=-2 \partial \bar{\partial} g_{s}=\partial \bar{\partial} \log \left(\|s\|^{2}\right)
$$

the first Chern form of $(L, h)$. We note that this definition of the first Chern form differs by a factor $(2 \pi \mathrm{i})^{-1}$ from the corresponding definition in cohomology with real coefficients because of the $2 \pi \mathrm{i}$-twist in Deligne-Beilinson cohomology.

Proposition 5.64. With the above assumptions the following statements hold. The form $\omega_{s}$ belongs to $\mathcal{D}_{\log }^{2}(X, 1)$, and the form $g_{s}$ belongs to $\mathcal{D}_{\log }^{1}(U, 1)$. The pair $\left(\omega_{s}, g_{s}\right)$ is a cycle of the simple complex

$$
s^{2}\left(\mathcal{D}_{\log }(X, 1) \rightarrow \mathcal{D}_{\log }(U, 1)\right) .
$$

Moreover, this pair represents the class of $\operatorname{div}(s)$ in the cohomology group $H_{\mathcal{D}, Y}^{2}(X, \mathbb{R}(1))$.

Proof. Let $s^{\prime}$ be a non-vanishing, regular section of $L$ in an open subset $V$ of $X$. Then, $\omega_{s}=\partial \bar{\partial} \log \left(\left\|s^{\prime}\right\|^{2}\right)$, which shows that $\omega_{s}$ is smooth on the whole of $\bar{X}$. Furthermore, since $g_{s}$ is a real function and $-2 \partial \bar{\partial}$ is an imaginary operator, we obtain

$$
\omega_{s} \in E_{\bar{X}, \mathbb{R}}^{2}(1) \cap E_{\bar{X}}^{1,1}=\mathcal{D}^{2}\left(E_{\bar{X}}, 1\right) \subseteq \mathcal{D}_{\log }^{2}(X, 1) .
$$

Let $\widetilde{X}$ be an embedded resolution of singularities of $\bar{Y}$, and $E$ the pre-image of $\bar{Y}$ in $\widetilde{X}$. If $E$ is locally described by the equation $z_{1} \cdots z_{m}=0$, the section $s$ can locally be written as $z_{1}^{\alpha_{1}} \cdots z_{m}^{\alpha_{m}} \cdot s^{\prime}$, where $s^{\prime}$ is a suitable non-vanishing, regular section on $\widetilde{X}$. This shows

$$
g_{s} \in E_{\log , \mathbb{R}}^{0}(U)=\mathcal{D}_{\log }^{1}(U, 1) .
$$

The claim that $\left(\omega_{s}, g_{s}\right)$ is a cycle follows from the definitions. The Poincaré-Lelong formula

$$
-2 \partial \bar{\partial}\left[g_{s}\right]_{X}=\left[\omega_{s}\right]-\delta_{\operatorname{div}(s)}
$$

together with Proposition 5.58 (i) finally proves that the pair $\left(\omega_{s}, g_{s}\right)$ represents the class of $\operatorname{div}(s)$.

\subsection{Real varieties}

\section{Real Deligne-Beilinson cohomology of real varieties}

Recall that a real variety $X_{\mathbb{R}}$ is a pair $\left(X_{\mathbb{C}}, F_{\infty}\right)$ with $X_{\mathbb{C}}$ a variety over $\mathbb{C}$ and $F_{\infty}$ an antilinear involution of $X_{\mathbb{C}}$. Analogously, a real algebraic manifold is a pair $\left(X_{\mathbb{C}}, F_{\infty}\right)$ with $X_{\mathbb{C}}$ a complex algebraic manifold. 
Notation 5.65. Given a real variety $X_{\mathbb{R}}$, and a sheaf $V$ of complex vector spaces with a real structure, we will denote by $\sigma$ the involution given by

$$
\omega \mapsto \overline{F_{\infty}^{*}(\omega)}
$$

We will use the same notation for any subsheaf of abelian groups of $V$, which is invariant under complex conjugation.

Let $W_{\mathbb{R}}$ now be a proper real algebraic manifold, and $D_{\mathbb{R}}$ a normal crossing divisor in $W_{\mathbb{R}}$ defined over $\mathbb{R}$. We put $X_{\mathbb{R}}=W_{\mathbb{R}} \backslash D_{\mathbb{R}}$. The antilinear involution $\sigma$ of $E_{W_{\mathbb{C}}}^{*}\left(\log D_{\mathbb{C}}\right)$ respects the real structure and the Hodge filtration. It thus induces an involution of $\mathcal{D}^{*}\left(E_{W_{\mathbb{C}}}\left(\log D_{\mathbb{C}}\right), p\right)$, which we denote again by $\sigma$. The real Deligne-Beilinson cohomology groups of $X_{\mathbb{R}}$ are defined as the cohomology of the complex of fixed elements $\mathcal{D}^{*}\left(E_{W_{\mathbb{C}}}\left(\log D_{\mathbb{C}}\right), p\right)^{\sigma}$, i.e.

$$
H_{\mathcal{D}}^{*}\left(X_{\mathbb{R}}, \mathbb{R}(p)\right)=H^{*}\left(\mathcal{D}\left(E_{W_{\mathbb{C}}}\left(\log D_{\mathbb{C}}\right), p\right)^{\sigma}\right) .
$$

An analogous definition can be given for the real Deligne-Beilinson cohomology groups with support. From the corresponding result for complex varieties, we obtain the following theorem.

Theorem 5.66. Real Deligne-Beilinson cohomology is a Gillet cohomology for regular real schemes.

\section{A Gillet complex for real Deligne-Beilinson cohomology}

Here we denote by $C$ the site of regular schemes in $\mathrm{ZAR}(\operatorname{Spec}(\mathbb{R}))$. A scheme $X$ in $C$ defines a real algebraic manifold $X_{\mathbb{R}}=\left(X_{\mathbb{C}}, F_{\infty}\right)$.

Definition 5.67. For any integers $n, p$, let $\mathcal{D}_{\log }^{n}(p)$ denote the presheaf (in fact, the sheaf) over $C$, which assigns to $X$ the group

$$
\mathcal{D}_{\log }^{n}(X, p)=\mathcal{D}^{n}\left(E_{\log }\left(X_{\mathbb{R}}(\mathbb{C})\right), p\right)^{\sigma}
$$

with $\sigma$ as in Notation 5.65. For any scheme $X$ in $C$, we will denote the induced presheaf of graded complexes of real vector spaces on $X$ by $\mathcal{D}_{\log , X}=\mathcal{D}_{\log , X}^{*}(*)$.

Proposition 5.68. Let $X$ be a scheme in $C$. For any integers $n, p$, the presheaf $\mathcal{D}_{\log , X}^{n}(p)$ is a totally acyclic sheaf.

Proof. Since we are working with complexes of vector spaces, the functor $(\cdot)^{\sigma}$ is exact. Therefore, the result follows from Proposition 5.33.

Theorem 5.69. The graded complex of sheaves of abelian groups $\mathcal{D}_{\log }$ is a Gillet complex for regular schemes over $\mathbb{R}$, which computes real Deligne-Beilinson cohomology. Moreover, the pair $\left(\mathcal{D}_{\log }, \bullet\right)$ is a graded commutative and pseudo-associative algebra for real Deligne-Beilinson cohomology.

Proof. This is a consequence of Theorem 5.34 and the fact that all operations are compatible with the involution $\sigma$. 


\section{Examples of $\mathcal{D}_{\log }$-arithmetic Chow groups}

In this section we will use the abstract theory of arithmetic Chow groups to define contravariant and covariant arithmetic Chow groups. The former were introduced in $[\mathbf{1 7}]$; they have a ring structure after tensoring with $\mathbb{Q}$ and agree with the arithmetic Chow groups defined by Gillet and Soulé in [36] for arithmetic varieties with projective generic fibre. The latter were introduced in [14]; they are covariant for arbitrary proper morphisms and have a module structure over the contravariant Chow groups.

In all the examples in this paper the complex $\mathcal{D}_{\log }$ will play the role of the Gillet complex, i.e. $\mathcal{G}=\mathcal{D}_{\log }$.

\subsection{Contravariant $\mathcal{D}_{\log }$-arithmetic Chow rings}

Definition and exact sequences

Let $A$ be an arithmetic ring. A natural example of a $\mathcal{D}_{\log }$-complex is $\mathcal{D}_{\text {log }}$ itself by the identity morphism. Then, all the properties of $\mathcal{D}_{\log }$ as a Gillet complex (multiplicativity, functoriality) imply the same properties for $\mathcal{D}_{\log }$ as a $\mathcal{D}_{\log }$-complex.

For any arithmetic variety $X$ over $A$, we put $\mathcal{G}=\mathcal{D}_{\log }$ and $\mathcal{C}=\mathcal{D}_{\log , X}$ in Definition 4.4. Thus the pair $\left(X, \mathcal{D}_{\log }\right)$ is a $\mathcal{D}_{\log }$-arithmetic variety over $A$. By means of Definition 4.11, we obtain the arithmetic Chow groups $\widehat{\mathrm{CH}}^{p}\left(X, \mathcal{D}_{\log }\right)$. We recover the properties of these groups by applying the theory developed in $\S 4$; these properties have already been stated in [17]. We start by writing down the exact sequences of Theorem 4.13. For this we recall

$$
\begin{aligned}
\widetilde{\mathcal{D}}_{\log }^{2 p-1}(X, p) & =\mathcal{D}_{\log }^{2 p-1}(X, p) / \operatorname{Im}\left(\mathrm{d}_{\mathcal{D}}\right) \\
& =\left\{\omega \in E_{\log , \mathbb{R}}^{p-1, p-1}\left(X_{\infty}\right)(p-1) \mid F_{\infty}^{*}(\omega)=\bar{\omega}\right\} /(\operatorname{Im} \partial+\operatorname{Im} \bar{\partial}) .
\end{aligned}
$$

Theorem 6.1. For an arithmetic variety $X$ over $A$, there are exact sequences:

$$
\begin{aligned}
& \mathrm{CH}^{p-1, p}(X) \stackrel{\rho}{\rightarrow} \widetilde{\mathcal{D}}_{\log }^{2 p-1}(X, p) \stackrel{\text { a }}{\rightarrow} \widehat{\mathrm{CH}}^{p}\left(X, \mathcal{D}_{\log }\right) \stackrel{\zeta}{\rightarrow} \mathrm{CH}^{p}(X) \rightarrow 0, \\
& \mathrm{CH}^{p-1, p}(X) \stackrel{\rho}{\rightarrow} H_{\mathcal{D}}^{2 p-1}\left(X_{\mathbb{R}}, \mathbb{R}(p)\right) \stackrel{\text { a }}{\rightarrow} \widehat{\mathrm{CH}}^{p}\left(X, \mathcal{D}_{\log }\right) \\
& \qquad \stackrel{(\zeta,-\omega)}{\rightarrow} \mathrm{CH}^{p}(X) \oplus \mathrm{ZD}_{\log }^{2 p}(X, p) \stackrel{\mathrm{cl}+h}{\rightarrow} H_{\mathcal{D}}^{2 p}\left(X X_{\mathbb{R}}, \mathbb{R}(p)\right) \rightarrow 0, \\
& \mathrm{CH}^{p-1, p}(X) \stackrel{\rho}{\rightarrow} H_{\mathcal{D}}^{2 p-1}\left(X_{\mathbb{R}}, \mathbb{R}(p)\right) \stackrel{\text { a }}{\rightarrow} \widehat{\mathrm{CH}}^{p}\left(X, \mathcal{D}_{\log }\right)_{0} \stackrel{\zeta}{\rightarrow} \mathrm{CH}^{p}(X)_{0} \rightarrow 0 .
\end{aligned}
$$

\section{Green forms for a cycle}

We now translate the result of Proposition 5.58 into the language of Green objects.

Proposition 6.5. Let $X$ be an arithmetic variety over $A$, and $y$ a $p$-codimensional cycle on $X$. A pair $\mathfrak{g}=(\omega, \widetilde{g}) \in \widehat{H}_{\mathcal{D}_{\log }, \mathcal{Z}^{p}}^{2 p}(X, p)$ is a Green object for the class of $y$, if and only if

$$
-2 \partial \bar{\partial}[g]_{X}=[\omega]-\delta_{y}
$$


To simplify notation, in the above proposition, we wrote $[g]_{X}$ instead of $[g]_{X_{\infty}}$, and $\delta_{y}$ instead of $\delta_{y_{\mathbb{R}}}$. We will call Green objects with values in $\mathcal{D}_{\log }$ Green forms.

The arithmetic cycle associated to a rational function

We make the Green object and the arithmetic cycle associated to a rational function explicit.

Proposition 6.7. Let $f \in k^{*}(X), y=\operatorname{div}(f), Y=\operatorname{supp} y$, and $U=X \backslash Y$. Then, the class $\mathrm{cl}_{\mathcal{D}}(f)$ of $f$ in the group $H_{\mathcal{D}_{\log }}^{1}(U, 1)=H_{\mathcal{D}}^{1}\left(U_{\mathbb{R}}, \mathbb{R}(1)\right)$ is represented by the function

$$
\frac{1}{2} \log (f \bar{f}) \in \mathcal{D}_{\log }^{1}(U, 1) ;
$$

we note that in order to ease notation, we have written $\operatorname{cl}_{\mathcal{D}}(f)$ instead of $\operatorname{cl}_{\mathcal{D}_{\log }}(f)$. Furthermore, we have

$$
\widehat{\operatorname{div}}(f)=\left(\operatorname{div}(f),\left(0,-\frac{1}{2} \log (f \bar{f})\right)\right) .
$$

Proof. Since we are interested in the cohomology of $U$, we may assume that $Y$ is a normal crossing divisor; we denote the inclusion by $j: U \rightarrow X$. Then, $f$ is a global section of $\mathcal{O}_{\text {alg }}^{\times}$over $U$.

To prove the first assertion, we have to show that the morphism

$$
H^{0}\left(U, \mathcal{O}_{\text {alg }}^{\times}\right) \rightarrow H_{\mathcal{D}}^{1}\left(U_{\mathbb{R}}, \mathbb{R}(1)\right),
$$

given by mapping $f$ to $\frac{1}{2} \log (f \bar{f})$, is compatible with the map $c_{1}$ of $(5.5)$. The key ingredient for this compatibility is provided by the commutative diagram of sheaves on $U$

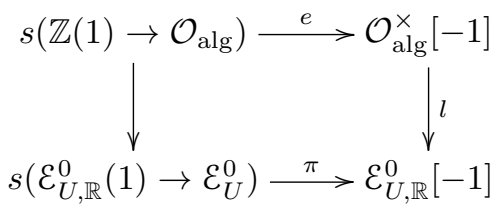

where $e(a, b)=\exp (b), l(f)=\frac{1}{2} \log (f \bar{f})$, and $\pi(a, b)=\frac{1}{2}(b+\bar{b})$. Denoting by $G$ the complex of sheaves on $U$ given by

$$
\mathcal{O}_{\text {alg }}^{\times} \stackrel{\mathrm{d} \log }{\longrightarrow} \Omega_{U}^{1} \rightarrow \Omega_{U}^{2} \rightarrow \cdots,
$$

the commutative diagram (6.7) induces a commutative diagram of sheaves on $X$

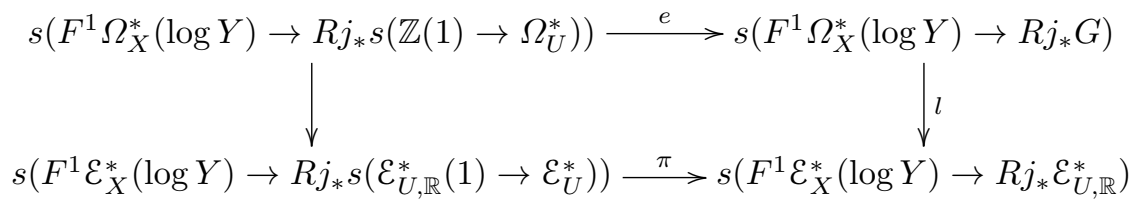

This commutative diagram shows that the class $\mathrm{cl}_{\mathcal{D}}(f)=\mathrm{c}_{1}(f)$ is represented by the pair $\left(\mathrm{d} \log f, \frac{1}{2} \log (f \bar{f})\right)$ in the complex $s\left(F^{1} \mathcal{E}_{X}^{*}(\log Y) \rightarrow R j_{*} \mathcal{E}_{U, \mathbb{R}}^{0}\right)$. This implies that the class $\operatorname{cl}_{\mathcal{D}}(f)$ is represented by $\frac{1}{2} \log (f \bar{f})$ in the complex $\mathcal{D}_{\log }^{*}(U, 1)$.

The second assertion follows from the first and the fact that the map $b$ is given by $\mathrm{b}(b)=(0,-b)($ see Definition 3.24). 
Multiplicative properties

By Theorem 5.69, the complex $\mathcal{D}_{\log }$ satisfies all the properties required to apply Theorem 4.24. In particular, we obtain the following theorem.

Theorem 6.9. The direct sum

$$
\widehat{\mathrm{CH}}^{*}\left(X, \mathcal{D}_{\log }\right)_{\mathbb{Q}}=\bigoplus_{p} \widehat{\mathrm{CH}}^{p}\left(X, \mathcal{D}_{\log }\right)_{\mathbb{Q}}
$$

has the structure of a commutative and associative $\mathbb{Q}$-algebra with unit. This structure is compatible with the maps $\omega, \mathrm{cl}$ and $\zeta$; furthermore, it is compatible with the algebra structures of the Deligne complex, the Deligne-Beilinson cohomology and the Chow ring.

Moreover, we observe that Theorem 4.25 and Corollary 4.26 also apply to the present situation. We leave it to the reader to write down the corresponding statements.

Formulae for the $*$-product

Using partitions of unity we will give alternative formulae for the *-product of Green objects. These intermediate formulae will be useful for the explicit computations carried out in our applications.

Let $y$ be a $p$-codimensional, respectively $z$ a $q$-codimensional, cycle of $X$, and $Y=$ $\operatorname{supp} y_{\mathbb{R}}$, respectively $Z=\operatorname{supp} z_{\mathbb{R}}$. Let $\mathfrak{g}_{y}=\left(\omega_{y}, \widetilde{g}_{y}\right) \in \widehat{H}_{\mathcal{D}_{\log }, Y}^{2 p}(X, p)$, respectively $\mathfrak{g}_{z}=$ $\left(\omega_{z}, \widetilde{g}_{z}\right) \in \widehat{H}_{\mathcal{D}_{\log }, Z}^{2 q}(X, q)$, be Green objects for $y$, respectively $z$.

\section{Proposition 6.10.}

(i) If $\mathfrak{g}_{y}=\mathrm{a}(\widetilde{x})$ for some $\widetilde{x} \in \widetilde{\mathcal{D}}_{\log }^{2 p-1}(X, p)$, we have the equality

$$
\mathfrak{g}_{y} * \mathfrak{g}_{z}=\mathrm{a}\left(\widetilde{x \wedge \omega_{z}}\right)
$$

in the group $\widehat{H}_{\mathcal{D}_{\log , Y \cap Z}^{2 p+2 q}}^{2 q}(X, p+q)$.

(ii) If $Y=Z$, we have the equality

$$
\mathfrak{g}_{y} * \mathfrak{g}_{z}=\left(\omega_{y} \wedge \omega_{z}, \widetilde{g_{y} \wedge \omega_{z}}\right)
$$

in the group $\widehat{H}_{\mathcal{D}_{\log }, Y}^{2 p+2 q}(X, p+q)$.

Proof. The first claim is an immediate consequence of Proposition 2.67 (iii) and the explicit formulae for the product in Deligne algebras given after Remark 5.16. The second claim is covered by the explicit formula for the *-product given in Proposition 2.68, again taking into account the formulae for the product in Deligne algebras given after Remark 5.16.

The cases $Y \subsetneq Z$ and $Z \subsetneq Y$ can be treated similarly. But the most interesting case is when neither $Y$ nor $Z$ are contained in $Y \cap Z$. In this case, we put $U=X \backslash Y$ and $V=X \backslash Z$. Then, the Mayer-Vietoris sequence

$$
0 \rightarrow \mathcal{D}_{\log }^{n}(U \cup V, p) \stackrel{i}{\rightarrow} \mathcal{D}_{\log }^{n}(U, p) \oplus \mathcal{D}_{\log }^{n}(V, p) \stackrel{j}{\rightarrow} \mathcal{D}_{\log }^{n}(U \cap V, p) \rightarrow 0,
$$


where $i(\eta)=(\eta, \eta)$ and $j(\omega, \eta)=-\omega+\eta$, gives rise to the kernel-simple quasiisomorphism

$$
\iota: \mathcal{D}_{\log }(U \cup V, p) \rightarrow s(-j)
$$

which was an essential tool in the proof of Theorem 3.31. We will now construct a section of $j$. Adapting the argument given in [16], we can find a resolution of singularities $\pi: \tilde{X} \rightarrow X_{\mathbb{R}}$ of $Y \cup Z$, which factors through embedded resolutions of $Y, Z, Y \cap Z$. In particular, we can assume that

$$
\pi^{-1}(Y), \pi^{-1}(Z), \pi^{-1}(Y \cap Z)
$$

are also normal crossing divisors. We denote by $\widehat{Y}$ the normal crossing divisor formed by the components of $\pi^{-1}(Y)$ which are not contained in $\pi^{-1}(Y \cap Z)$; analogously we denote by $\widehat{Z}$ the normal crossing divisor formed by the components of $\pi^{-1}(Z)$ which are not contained in $\pi^{-1}(Y \cap Z)$. Then, $\widehat{Y}$ and $\widehat{Z}$ are closed subsets of $\widetilde{X}$ which do not meet. Therefore, there exist two smooth, $F_{\infty}$-invariant functions $\sigma_{Y Z}$ and $\sigma_{Z Y}$ satisfying $0 \leqslant \sigma_{Y Z}, \sigma_{Z Y} \leqslant 1, \sigma_{Y Z}+\sigma_{Z Y}=1, \sigma_{Y Z}=1$ in a neighbourhood of $\widehat{Y}$, and $\sigma_{Z Y}=1$ in a neighbourhood of $\widehat{Z}$. Let now $\omega \in E_{\log }^{n}(U \cap V)$. Since $\sigma_{Y Z}$ is zero in a neighbourhood of $\widehat{Z}$, we find that $\sigma_{Y Z} \omega \in E_{\log }^{n}(U)$; similarly, we get $\sigma_{Z Y} \omega \in E_{\log }^{n}(V)$. Moreover, one easily checks that $j\left(-\sigma_{Y Z} \omega, \sigma_{Z Y} \omega\right)=\omega$. Therefore, the assignment $\omega \mapsto\left(-\sigma_{Y Z} \omega, \sigma_{Z Y} \omega\right)$ gives rise to a section of $j$. Consequently, the map $\mathcal{D}_{\log }^{n}(U \cap V, p) \rightarrow \mathcal{D}_{\log }^{n}(U, p) \oplus \mathcal{D}_{\log }^{n}(V, p)$ given by $c \mapsto\left(-\sigma_{Y Z} c, \sigma_{Z Y} c\right)$ determines a section of $j$. We are now in a position to apply Proposition 2.41 (ii).

Lemma 6.11. The map $s(-j) \rightarrow \mathcal{D}_{\log }(U \cup V, p)$ given by

$$
((a, b), c) \mapsto \sigma_{Z Y} a+\sigma_{Y Z} b+\sigma_{Y Z} \mathrm{~d}_{\mathcal{D}} c-\mathrm{d}_{\mathcal{D}}\left(\sigma_{Y Z} c\right)
$$

is a morphism of complexes. It is a left inverse of the kernel-simple quasi-isomorphism $\iota$.

Theorem 6.12. Let $\mathfrak{g}_{y}=\left(\omega_{y}, \widetilde{g}_{y}\right), \mathfrak{g}_{z}=\left(\omega_{z}, \widetilde{g}_{z}\right)$, and $\sigma_{Y Z}, \sigma_{Z Y}$ be as above. In the group $\widehat{H}_{\mathcal{D}_{\log }, Y \cap Z}^{2 p+2 q}(X, p+q)$, we then have the identity

$$
\mathfrak{g}_{y} * \mathfrak{g}_{z}=\left(\omega_{y} \wedge \omega_{z},\left(-2 \sigma_{Z Y} g_{y} \wedge \partial \bar{\partial} g_{z}-2 \partial \bar{\partial}\left(\sigma_{Y Z} g_{y}\right) \wedge g_{z}\right)^{\sim}\right) .
$$

Proof. By Theorem 3.37, we obtain

$$
\mathfrak{g}_{y} * \mathfrak{g}_{z}=\left(\omega_{y} \bullet \omega_{z},\left(\left(g_{y} \bullet \omega_{z}, \omega_{y} \bullet g_{z}\right),-g_{y} \bullet g_{z}\right)^{\sim}\right)
$$

in the group $\widehat{H}^{2 p+2 q}\left(\mathcal{D}_{\log }(X), s(-j), p+q\right)$. By means of Lemma 6.11, the latter element corresponds to

$$
\left(\omega_{y} \bullet \omega_{z},\left(\sigma_{Z Y} g_{y} \bullet \mathrm{d}_{\mathcal{D}} g_{z}+\mathrm{d}_{\mathcal{D}}\left(\sigma_{Y Z} g_{y}\right) \bullet g_{z}\right)^{\sim}\right) \in \widehat{H}_{\mathcal{D}_{\log }, Y \cap Z}^{2 p+2 q}(X, p+q) .
$$

The stated formula follows now from the explicit description of $\bullet$ and $d_{\mathcal{D}}$ in these degrees. 
Inverse images

Let $f: X \rightarrow Y$ be a morphism of arithmetic varieties over $A$. Since $\mathcal{D}_{\log }$ is a sheaf in the big Zariski site of smooth schemes over $\mathbb{R}$, there exists a contravariant $f$-morphism

$$
f^{\#}: \mathcal{D}_{\log , Y} \rightarrow f_{*} \mathcal{D}_{\log , X}
$$

Thus, the complex $\mathcal{D}_{\log }$ satisfies all the properties required to apply Theorems 4.29 and 4.31. In particular, we obtain the following theorem.

Theorem 6.13. Let $f: X \rightarrow Y$ be a morphism of arithmetic varieties over $A$. Then, there is a pull-back morphism

$$
f^{*}: \widehat{\mathrm{CH}}^{p}\left(Y, \mathcal{D}_{\log }\right) \rightarrow \widehat{\mathrm{CH}}^{p}\left(X, \mathcal{D}_{\log }\right),
$$

which is compatible with the pull-back of differential forms via the morphism $\omega$, and with the pull-back of algebraic cycles via the morphism $\zeta$.

If $g: Y \rightarrow Z$ is another such morphism, then the equality $(g \circ f)^{*}=f^{*} \circ g^{*}$ holds.

Moreover, the induced map

$$
f^{*}: \widehat{\mathrm{CH}}^{p}\left(Y, \mathcal{D}_{\log }\right)_{\mathbb{Q}} \rightarrow{\widehat{\mathrm{CH}^{p}}}^{p}\left(X, \mathcal{D}_{\log }\right)_{\mathbb{Q}}
$$

is an algebra morphism.

\section{Direct images of differential forms with logarithmic singularities}

Differential forms with logarithmic singularities at infinity are not well suited to define covariant morphisms for $\mathcal{D}_{\log }$-complexes, as the following example shows.

Example 6.14. Let $f: C \rightarrow C^{\prime}$ be a morphism of smooth complex projective curves, and let $\Sigma$ be the pre-image of the singular values of the map $f$. Assume that there is a point $P \in \Sigma$ such that the morphism $f$ is given by the local expression $w=f(z)=z^{2}$ in an analytic neighbourhood of $P$. While the differential form $\mathrm{d} z \wedge \mathrm{d} \bar{z}$ is smooth in a neighbourhood of $P$, its push-forward $f_{*}(\mathrm{~d} z \wedge \mathrm{d} \bar{z})$ is locally of the form $\mathrm{d} w \wedge \mathrm{d} \bar{w} /(w \bar{w})^{1 / 2}$, which is not smooth and does not have logarithmic singularities. This shows that, even if the restriction

$$
f: C \backslash \Sigma \rightarrow C^{\prime} \backslash f(\Sigma)
$$

is smooth, it does not induce a morphism between $E_{\log }^{*}(C \backslash \Sigma)$ and $E_{\log }^{*}\left(C^{\prime} \backslash f(\Sigma)\right)$.

Nevertheless, if $f: X \rightarrow Y$ is a smooth proper equidimensional morphism of projective varieties over $\mathbb{R}$ of relative dimension $e$, we can define a covariant $f$-pseudo-morphism as follows. For every open subset $U$ of $Y$ we define

$$
\mathcal{F}^{n}(U, p)=\underset{\bar{Y}, D}{\lim _{\vec{Y}}} \mathcal{D}^{n}\left(E_{X \times \bar{Y}}(\log X \underset{Y}{\times} D), p\right),
$$

where the limit is taken over all compactifications $\bar{Y}$ of $U$ with $D=\bar{Y} \backslash U$ a normal crossing divisor such that $\bar{Y}$ dominates $Y$. Since $f$ is smooth, we note that $X \underset{Y}{X} D$ is a normal crossing divisor of the smooth variety $\underset{Y}{\times} \bar{Y}$. Let

$$
u: \mathcal{F} \rightarrow f_{*} \mathcal{D}_{\log , X}
$$


be the natural morphism, and let

$$
v: \mathcal{F} \rightarrow \mathcal{D}_{\log , Y}(-e)[-2 e]
$$

be the morphism induced by integration along the fibre, i.e.

$$
v(\omega)=f_{!} \omega=\frac{1}{(2 \pi \mathrm{i})^{e}} \int_{f} \omega .
$$

By Proposition 5.56 the morphisms $u$ and $v$ determine a covariant $f$-pseudo-morphism. Using Definition 3.73 together with Remarks 3.74 and 3.79 and Proposition 3.76 we obtain the following theorem.

Theorem 6.15. Let $f: X \rightarrow Y$ be a smooth proper morphism of proper varieties over $\mathbb{R}$ of relative dimension $e$. Then, there is a push-forward morphism

$$
f_{\#}: \widehat{H}_{\mathcal{D}_{\log }, \mathcal{Z}^{p}}^{2 p}(X, p) \rightarrow \widehat{H}_{\mathcal{D}_{\log }, \mathcal{Z}^{p-e}}^{2 p-2 e}(Y, p-e),
$$

which is compatible with the push-forward of differential forms via the morphism $\omega$, and with the direct image into relative Deligne-Beilinson cohomology via the morphism cl.

If $g: Y \rightarrow Z$ is another such morphism, then the equality $(g \circ f)_{\#}=g_{\#} \circ f_{\#}$ holds.

Moreover, if $\alpha \in \widehat{H}_{\mathcal{D}_{\text {log }}, \mathcal{Z}^{p}}^{2 p}(Y, p)$ and $\beta \in \widehat{H}_{\mathcal{D}_{\text {log }}, \mathcal{Z}^{q}}^{2 q}(X, q)$, we have the formula

$$
f_{\#}\left(f^{\#}(\alpha) \cdot \beta\right)=\alpha \cdot f_{\#}(\beta) \in \widehat{H}_{\mathcal{D}_{\log }^{2 p+2 q-2 e}, \mathcal{Z}^{p+q-e}}(Y, p+q-e) .
$$

Remark 6.16. In order to obtain more general push-forward morphisms one has to use different complexes. For instance, using a real Deligne-Beilinson complex made with differential forms satisfying logarithmic growth conditions instead of logarithmic singularities at infinity, we expect that one can avoid the projectivity condition for the varieties under consideration. Another option is the use of the real Deligne-Beilinson complex with currents as carried out in the next section; then, one obtains direct images for arbitrary proper morphisms, at the price of losing some multiplicativity properties.

\section{Direct images of contravariant arithmetic Chow rings}

Using the technique of covariant pseudo-morphisms as before, we can define a pushforward morphism associated to a morphism between arithmetic varieties, which are generically projective and smooth. As we will see below, this suffices to define arithmetic degrees. The following result is a consequence of Theorem 6.15 and Theorem 4.34.

Theorem 6.17. Let $f: X \rightarrow Y$ be a proper morphism of arithmetic varieties over $A$ of relative dimension e. Assume that $f_{\mathbb{R}}: X_{\mathbb{R}} \rightarrow Y_{\mathbb{R}}$ is a smooth proper morphism of proper varieties. Then, there is a push-forward morphism

$$
f_{*}: \widehat{\mathrm{CH}}^{p}\left(X, \mathcal{D}_{\log }\right) \rightarrow \widehat{\mathrm{CH}}^{p-e}\left(Y, \mathcal{D}_{\log }\right),
$$

which is compatible with the push-forward of differential forms via the morphism $\omega$, and with the push-forward of algebraic cycles via the morphism $\zeta$. 
If $g: Y \rightarrow Z$ is another such morphism, then the equality $(g \circ f)_{*}=g_{*} \circ f_{*}$ holds.

Moreover, if $\alpha \in \widehat{\mathrm{CH}}^{p}\left(Y, \mathcal{D}_{\log }\right)$ and $\beta \in \widehat{\mathrm{CH}}^{q}\left(X, \mathcal{D}_{\log }\right)$, we have the formula

$$
f_{*}\left(f^{*}(\alpha) \cdot \beta\right)=\alpha \cdot f_{*}(\beta) \in \widehat{\mathrm{CH}}^{p+q-e}\left(Y, \mathcal{D}_{\log }\right)_{\mathbb{Q}} \cdot
$$

\section{Homotopy invariance}

The fact that the definition of contravariant arithmetic Chow groups for non-proper varieties has good Hodge theoretical properties is reflected in the homotopy invariance of the groups $\widehat{\mathrm{CH}}^{p}\left(X, \mathcal{D}_{\log }\right)_{0}$.

Proposition 6.18. Let $X$ be an arithmetic variety over $A$, and $\pi: M \rightarrow X$ a geometric vector bundle. Then, the induced morphism

$$
\pi^{*}: \widehat{\mathrm{CH}}^{p}\left(X, \mathcal{D}_{\log }\right)_{0} \rightarrow \widehat{\mathrm{CH}}^{p}\left(M, \mathcal{D}_{\log }\right)_{0}
$$

is an isomorphism.

Proof. For a proof we refer to $[\mathbf{1 7}, 7.5]$.

This theorem has the following variant used by $\mathrm{Hu}$ in his $\mathrm{PhD}$ thesis to construct an arithmetic intersection pairing based on the deformation to the normal cone technique. With the hypothesis of the above theorem, we write

$$
\mathcal{D}_{\log }^{*}(M, p)_{\text {vert }}=\pi^{*}\left(\mathcal{D}_{\log }^{*}(X, p)\right) \subseteq \mathcal{D}_{\log }^{*}(M, p),
$$

and put

$$
\widehat{\mathrm{CH}}^{p}\left(M, \mathcal{D}_{\log }\right)_{\text {vert }}=\omega^{-1}\left(\mathcal{D}_{\log }^{2 p}(M, p)_{\text {vert }}\right) .
$$

Then, we have the following proposition.

Proposition 6.19. Let $X$ be an arithmetic variety over $A$, and $\pi: M \rightarrow X$ a geometric vector bundle. Then, the induced morphism

$$
\pi^{*}: \widehat{\mathrm{CH}}^{p}\left(X, \mathcal{D}_{\log }\right) \rightarrow \widehat{\mathrm{CH}}^{p}\left(M, \mathcal{D}_{\log }\right)_{\text {vert }}
$$

is an isomorphism.

Comparison with the arithmetic Chow groups defined by Gillet and Soulé

Let $X$ be a $d$-dimensional arithmetic variety over $A$. We denote by $\widehat{\mathrm{CH}}^{p}(X)$ the arithmetic Chow groups defined by Gillet and Soulé. Let $y$ be a $p$-codimensional cycle of $X$, $Y=\operatorname{supp} y_{\mathbb{R}}, U=X_{\mathbb{R}} \backslash Y$, and

$$
\mathfrak{g}_{y}=\left(\omega_{y}, \widetilde{g}_{y}\right) \in \widehat{H}^{2 p}\left(\mathcal{D}_{\log }\left(X_{\mathbb{R}}, p\right), \mathcal{D}_{\log }(U, p)\right)
$$

a Green object for the cycle $y$. 
Lemma 6.20. The current $2(2 \pi \mathrm{i})^{d-p+1}\left[g_{y}\right]_{X}$ is a Green current in the sense of [36] for the cycle $y$.

Proof. Since the definitions of the current associated to a differential form and the current associated to a cycle used in this paper differ from those in [36] by a normalization factor, we will write the proof explicitly in terms of integrals.

We may assume that the arithmetic ring $A$ is $\mathbb{R}$ and that $y$ is a prime cycle, hence $Y$ is irreducible. We denote by $\widetilde{Y}$ a resolution of singularities of $Y_{\infty}$, and by $\imath: \widetilde{Y} \rightarrow X_{\infty}$ the induced map.

A current $g \in D^{p-1, p-1}\left(X_{\infty}\right)$ is a Green current for the cycle $y$, if the equality

$$
F_{\infty}^{*}(g)=(-1)^{p-1} g
$$

holds, and if there exists a smooth differential form $\omega$ satisfying

$$
g\left(\mathrm{~d}^{\mathrm{c}} \eta\right)=\int_{X_{\infty}} \eta \wedge \omega-\int_{\widetilde{Y}} \imath^{*} \eta
$$

for any test form $\eta$.

We put $g=2(2 \pi \mathrm{i})^{d-p+1}\left[g_{y}\right]_{X}$, and fix a test form $\eta$. Then, the definition of the current associated to a differential form and the relation $(4 \pi \mathrm{i}) \mathrm{d}^{\mathrm{c}}=-2 \partial \bar{\partial}$ leads to

$$
g\left(\mathrm{~d}^{\mathrm{c}} \eta\right)=\frac{1}{(2 \pi \mathrm{i})^{p}} \int_{X_{\infty}}(-2 \partial \bar{\partial} \eta) \wedge g_{y} .
$$

Since $\sigma\left(g_{y}\right)=\overline{F_{\infty}^{*}\left(g_{y}\right)}=g_{y}$ and $\overline{g_{y}}=(-1)^{p-1} g_{y}$, we conclude that $F_{\infty}^{*}(g)=(-1)^{p-1} g$. Therefore, the first condition for a Green current is satisfied. Writing out explicitly all normalization factors in Proposition 6.5, we find

$$
\frac{1}{(2 \pi \mathrm{i})^{d}} \int_{X_{\infty}}(-2 \partial \bar{\partial} \eta) \wedge g_{y}=\frac{1}{(2 \pi \mathrm{i})^{d}} \int_{X_{\infty}} \eta \wedge \omega_{y}-\frac{1}{(2 \pi \mathrm{i})^{d-p}} \int_{\widetilde{Y}} \imath^{*} \eta .
$$

Equation (6.21) is now easily seen to imply that $g$ satisfies equation (6.20) with $\omega=$ $(2 \pi \mathrm{i})^{-p} \omega_{y}$, which concludes the proof of the lemma.

Theorem 6.23. The assignment $\left[y,\left(\omega_{y}, \widetilde{g}_{y}\right)\right] \mapsto\left[y, 2(2 \pi \mathrm{i})^{d-p+1}\left[g_{y}\right]_{X}\right]$ induces a welldefined map

$$
\Psi: \widehat{\mathrm{CH}}^{p}\left(X, \mathcal{D}_{\log }\right) \rightarrow \widehat{\mathrm{CH}}^{p}(X),
$$

which is compatible with products and pull-backs. Moreover, if $X_{\mathbb{R}}$ is projective, then it is an isomorphism, which is compatible with push-forwards.

Proof. In the projective case, the proof is given in [16] and [17]. On the other hand, we note that the well-definedness of the map and its compatibility with products and pull-backs carries over to the quasi-projective case. 


\section{Arithmetic Picard group}

Let $X$ be a projective arithmetic variety over $A$, and $L$ a line bundle on $X$ equipped with a smooth hermitian metric $h$ on the induced line bundle $L_{\infty}$ over $X_{\infty}$, which is invariant under $F_{\infty}$. As usual, we write $\bar{L}=(L,\|\cdot\|)$, and refer to it as a hermitian line bundle. Given a rational section $s$ of $L$, we write $\|s\|^{2}=h(s, s)$ for the point-wise norm of the induced section of $L_{\infty}$. We say that two hermitian line bundles $\bar{L}_{1}$ and $\bar{L}_{2}$ are isometric, if $\bar{L}_{1} \otimes \bar{L}_{2}^{-1} \cong\left(\mathcal{O}_{X},|\cdot|\right)$, where $|\cdot|$ is the standard absolute value. The arithmetic Picard group $\widehat{\operatorname{Pic}}(X)$ is the group of isometry classes of hermitian line bundles with group structure given by the tensor product. One easily proves that there is an isomorphism

$$
\widehat{\mathrm{c}}_{1}: \widehat{\operatorname{Pic}}(X) \rightarrow \widehat{\mathrm{CH}}^{1}\left(X, \mathcal{D}_{\log }\right),
$$

given by sending the class of $\bar{L}$ to the class $\left[\operatorname{div}(s),\left(\omega_{s}, \widetilde{g}_{s}\right)\right]$, where $s, \omega_{s}$, and $g_{s}$ are as in Proposition 5.64. We call the element

$$
\widehat{\mathrm{c}}_{1}(\bar{L}) \in \widehat{\mathrm{CH}}^{1}\left(X, \mathcal{D}_{\log }\right)
$$

the first arithmetic Chern class of $\bar{L}$.

\section{Arithmetic degree map}

Let $K$ be a number field, and $\mathcal{O}_{K}$ its ring of integers. According to [36], $\mathcal{O}_{K}$ can be viewed as an arithmetic ring in a canonical way. Putting $S=\operatorname{Spec}\left(\mathcal{O}_{K}\right)$, Theorem 6.23 provides an isomorphism

$$
\Psi: \widehat{\mathrm{CH}}^{1}\left(S, \mathcal{D}_{\log }\right) \cong \widehat{\mathrm{CH}}^{1}(S) .
$$

In particular, the computations of $[\mathbf{3 6}, 3.4 .3]$ carry over to this case with some minor changes. Since $S_{\infty}$ consists of a finite number of points, we have

$$
\widehat{H}_{\mathcal{D}_{\log }, \mathcal{Z}^{1}}^{2}(S, 1)=\left(\sum_{\sigma \in \Sigma} \mathbb{R}(0)\right)^{+}=\mathbb{R}^{r_{1}+r_{2}},
$$

where the superscript ' + ' denotes invariants under complex conjugation acting on the set of the $r_{1}$ real, respectively $2 r_{2}$, complex immersions of $K$. The exact sequence $(6.1)$ therefore specializes to the exact sequence

$$
1 \rightarrow \mu(K) \rightarrow \mathcal{O}_{K}^{*} \stackrel{\rho}{\rightarrow} \mathbb{R}^{r_{1}+r_{2}} \stackrel{\mathrm{a}}{\rightarrow} \widehat{\mathrm{CH}}^{1}\left(S, \mathcal{D}_{\log }\right) \rightarrow \mathrm{Cl}\left(\mathcal{O}_{K}\right) \rightarrow 0
$$

here $\mu(K)$ is the group of roots of unity of $K, \rho$ the Dirichlet regulator map, and $\mathrm{Cl}\left(\mathcal{O}_{K}\right)$ the ideal class group of $K$. We observe that, with the normalizations used in this paper, the map $\rho$ equals the Dirichlet regulator map and there is no factor of $(-2)$ as in $[36]$. The discrepancy by the factor 2 is explained by Theorem 6.23 ; the discrepancy about the sign is due to the fact that our map a is minus the corresponding map in [36].

Due to the product formula for the valuations of $K$, we have as in [36] a well-defined arithmetic degree map

$$
\widehat{\operatorname{deg}}: \widehat{\mathrm{CH}}^{1}\left(S, \mathcal{D}_{\log }\right) \rightarrow \mathbb{R},
$$


induced by the assignment

$$
\left(\sum_{\mathfrak{p}_{\mathfrak{j}} \in S} n_{j} \mathfrak{p}_{j}, \sum_{\sigma \in \Sigma}\left(0, \widetilde{g}_{\sigma}\right)\right) \mapsto \sum_{\mathfrak{p}_{j} \in S} n_{j} \log \left|\mathcal{O}_{K} / \mathfrak{p}_{j}\right|+\sum_{\sigma \in \Sigma} g_{\sigma}
$$

In particular, this is a group homomorphism, which is an isomorphism in the case $K=\mathbb{Q}$; it is common to identify $\widehat{\mathrm{CH}}^{1}\left(\operatorname{Spec}(\mathbb{Z}), \mathcal{D}_{\log }\right)$ with $\mathbb{R}$. We note that in spite of the many different normalizations used in this paper, the arithmetic degree map defined above is compatible with the arithmetic degree map defined in [36] under the isomorphism $\Psi$ of Theorem 6.23.

\subsection{Covariant $\mathcal{D}_{\text {log-arithmetic Chow groups }}$}

In this section we will use currents to define arithmetic Chow groups which are covariant for arbitrary proper morphisms. These groups do not have a ring structure, but they are modules over the contravariant arithmetic Chow ring defined in the previous section. We will grade the cycles by their codimension, and hence we will use the cieties $X$. Note however that we could also define covariant arithmetic Chow groups indexed by the dimension as in $[\mathbf{1 4}]$ avoiding this restriction.

\section{Currents with good Hodge properties}

We start by noting that, if $Y$ is a normal crossing divisor on a proper equidimensional complex algebraic manifold $X$, then the complex $\mathcal{D}_{X / Y}^{*}$ has the same cohomological properties as the complex $\mathcal{E}_{X}^{*}(\log Y)$. Nevertheless, we cannot use this complex of currents, since it does not form a presheaf. For instance, if $Y$ is a closed subvariety of $X$, and $\pi: \widetilde{X} \rightarrow X$ an embedded resolution of singularities of $Y$ with exceptional divisor $D$, there does not exist a natural morphism $\mathcal{D}_{X}^{*} \rightarrow \mathcal{D}_{\widetilde{X} / D}^{*}$, in general. For this reason the theory of covariant arithmetic Chow groups is not fully satisfactory.

\section{Currents with support on a subvariety}

Instead, we will use another complex of currents for quasi-projective subvarieties, which forms a presheaf, but has worse Hodge theoretical properties. To do this, let $X$ be a proper equidimensional complex algebraic manifold, and $\mathcal{D}_{X}^{*}$ the complex of currents on $X$ as in $\S 5.4$.

Definition 6.25. Let $Y \subseteq X$ be a closed subvariety. Then, the group of currents of degree $n$ on $X$ with support on $Y$ is defined by

$$
\mathcal{D}_{Y_{\infty}}^{n}=\left\{T \in \mathcal{D}_{X}^{n} \mid \operatorname{supp} T \subseteq Y\right\}
$$

we write

$$
\mathcal{D}_{X / Y_{\infty}}^{n}=\mathcal{D}_{X}^{n} / \mathcal{D}_{Y_{\infty}}^{n}
$$

We note that $\mathcal{D}_{Y_{\infty}}^{*}$ and $\mathcal{D}_{X / Y_{\infty}}^{*}$ are complexes, since supp $\mathrm{d} T \subseteq \operatorname{supp} T$. 
Invariance under birational morphisms

The main reason for using the complex $\mathcal{D}_{X / Y_{\infty}}^{*}$ is explained by the following result obtained by Poly (for a proof, see [66]).

Theorem 6.26. Let $f: X^{\prime} \rightarrow X$ be a proper morphism of equidimensional complex algebraic manifolds, and $Y \subsetneq X$ a closed subvariety satisfying $Y^{\prime}=f^{-1}(Y)$. If

$$
\left.f\right|_{X^{\prime} \backslash Y^{\prime}}: X^{\prime} \backslash Y^{\prime} \rightarrow X \backslash Y
$$

is an isomorphism, then the induced morphism

$$
f_{*}: \mathcal{D}_{X^{\prime} / Y_{\infty}^{\prime}}^{*} \rightarrow \mathcal{D}_{X / Y_{\infty}}^{*}
$$

is an isomorphism.

\section{Cohomological properties}

Let $X$ be a proper equidimensional complex algebraic manifold, $Y \subsetneq X$ a closed subvariety, and $U=X \backslash Y$. Since the complex $\overline{\mathcal{D}}_{Y}^{*}$ of currents on $Y$ is a subcomplex of $\mathcal{D}_{Y_{\infty}}^{*}$, there is an induced morphism $\mathcal{D}_{X / Y}^{*} \rightarrow \mathcal{D}_{X / Y_{\infty}}^{*}$; we note that these two complexes do not agree. As usual, we put

$$
D_{X / Y_{\infty}}^{*}=\Gamma\left(X, \mathcal{D}_{X / Y_{\infty}}^{*}\right),
$$

and observe that the complex $D_{X / Y_{\infty}}=\left(D_{X / Y_{\infty}, \mathbb{R}}^{*}, \mathrm{~d}\right)$ is a Dolbeault complex.

Proposition 6.27. For any integers $p, q$, the assignment which sends an open subset $U$ of $X$ to $D_{X / Y_{\infty}}^{p, q}$ (where $\left.Y=X \backslash U\right)$, is a totally acyclic sheaf.

Proof. Let $U$ and $V$ be open subsets of $X$. We put $Y=X \backslash U$ and $Z=X \backslash V$. Since the complexes of currents depend only on $U \cup V$ and not on $X$, we may assume that $Y=Y_{0} \cup W, Z=Z_{0} \cup W$ such that $Y_{0} \cap Z_{0}=\emptyset$ with closed subsets $Y_{0}, Z_{0}$, and $W$. We have to show that the sequence

$$
0 \rightarrow D_{(Y \cap Z)_{\infty}}^{p, q} \rightarrow D_{Y_{\infty}}^{p, q} \oplus D_{Z_{\infty}}^{p, q} \rightarrow D_{(Y \cup Z)_{\infty}}^{p, q} \rightarrow 0
$$

is exact. By definition, one obviously has

$$
D_{(Y \cap Z)_{\infty}}^{p, q}=D_{Y_{\infty}}^{p, q} \cap D_{Z_{\infty}}^{p, q}
$$

Letting $\left\{\sigma_{Y}, \sigma_{Z}\right\}$ be a partition of unity subordinate to the open covering $\left\{X \backslash Z_{0}, X \backslash Y_{0}\right\}$, and writing any current $T \in D_{(Y \cup Z)_{\infty}}^{p, q}$ as $T=\sigma_{Y} T+\sigma_{Z} T$, we see on the other hand that

$$
D_{(Y \cup Z)_{\infty}}^{p, q}=D_{Y_{\infty}}^{p, q}+D_{Z_{\infty}}^{p, q}
$$

This proves the proposition. 
Let now $\pi: \tilde{X} \rightarrow X$ be an embedded resolution of singularities of $Y$ with $D=\pi^{-1}(Y)$ a normal crossing divisor, i.e. $\widetilde{X}$ can be viewed as a smooth compactification $\bar{U}$ of $U$ with $D=\bar{U} \backslash U$ a normal crossing divisor. By means of Theorem 5.44 , we obtain a morphism of complexes

$$
E_{\bar{U}}^{*}(\log D) \rightarrow D_{\widetilde{X} / D}^{*} \rightarrow D_{\widetilde{X} / D_{\infty}}^{*}
$$

which leads, by Theorem 6.26, to a morphism of presheaves of Dolbeault complexes

$$
E_{\log }(U)^{\circ}=\left(E_{\log , \mathbb{R}}^{*}(U)^{\circ}, \mathrm{d}\right) \rightarrow\left(D_{X / Y_{\infty}, \mathbb{R}}^{*}, \mathrm{~d}\right)=D_{X / Y_{\infty}}
$$

which, in turn, induces a morphism of sheaves of Dolbeault complexes

$$
E_{\log }(U)=\left(E_{\log , \mathbb{R}}^{*}(U), \mathrm{d}\right) \rightarrow\left(D_{X / Y_{\infty}, \mathbb{R}}^{*}, \mathrm{~d}\right)=D_{X / Y_{\infty}}
$$

By another result of Poly (see [66]) this morphism is a quasi-isomorphism. Nevertheless, it is not a filtered quasi-isomorphism with respect to the Hodge filtration. The Hodge filtration of the Dolbeault complex $D_{X / Y_{\infty}}$ is related to the formal Hodge filtration studied by Ogus in [65].

Remark 6.29. We have now obtained a complex of currents on a quasi-projective variety which does not depend on the choice of a compactification. The price we have to pay is that the Hodge filtration of this complex is not the desired one. It would be useful to have a complex of currents on quasi-projective varieties which is independent on the compactification and compatible with the right Hodge filtration.

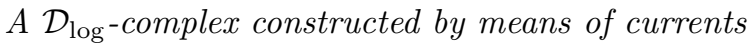

Let $C$ denote the site of regular schemes in $\operatorname{ZAR}(\operatorname{Spec}(\mathbb{R}))$. A scheme $X$ in $C$ defines a real algebraic manifold $X_{\mathbb{R}}=\left(X_{\mathbb{C}}, F_{\infty}\right)$.

Definition 6.30. Let $X$ be an equidimensional scheme in $C, U$ an open subset of $X$, $Y=X \backslash U$, and $\pi: \widetilde{X} \rightarrow X$ an embedded resolution of singularities of $Y$ with $D=\pi^{-1}(Y)$ a normal crossing divisor. For any integers $n, p$, let $\mathcal{D}_{\text {cur }, X}^{n}(p)=\mathcal{D}_{\text {cur }, X}^{n}(\cdot, p)$ denote the presheaf in the Zariski topology of $X$, which assigns to $U$ the group

$$
\mathcal{D}_{\text {cur }, X}^{n}(U, p)=\mathcal{D}^{n}\left(D_{\widetilde{X}_{\mathbb{C}} / D_{\mathbb{C} \infty}}, p\right)^{\sigma},
$$

with $\sigma$ as in Notation 5.65.

Proposition 6.31. Let $X$ be an equidimensional scheme in $C$. For any integers $n, p$, the presheaf $\mathcal{D}_{\text {cur }, X}^{n}(p)$ is a totally acyclic sheaf. Moreover, it has a natural structure of a $\mathcal{D}_{\log }$-complex.

Proof. The first statement is consequence of Proposition 6.27 and the exactness of the functors $\mathcal{D}^{n}(\cdot, p)$. The structure as a $\mathcal{D}_{\log }$-complex is given by the morphism (6.27). 


\section{Multiplicative properties}

Proposition 6.32. Let $X$ be an equidimensional scheme in $C$ of dimension $d$. Then,

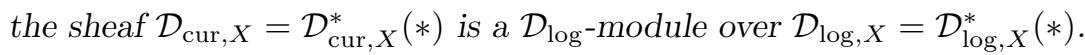

Proof. Let $U$ be an open subset of $X, Y=X \backslash U$, and $\pi: \widetilde{X} \rightarrow X$ an embedded resolution of singularities of $Y$ with $D=\pi^{-1}(Y)$ a normal crossing divisor. The space of currents $D_{\widetilde{X}_{\mathbb{C}} / D_{\mathbb{C} \infty}}^{n}$ is the topological dual of the space of differential forms of degree $2 d-n$ on $\widetilde{X}_{\mathbb{C}}$, which are flat along $D_{\mathbb{C}}$ (for a proof in the case $n=2 d$, see $[\mathbf{6 2}]$ ). Since the product of a form with logarithmic singularities along $D_{\mathbb{C}}$ with a form which is flat along $D_{\mathbb{C}}$, is again flat along $D_{\mathbb{C}}$ according to $[\mathbf{7 3}$, IV.4.2] there is a product

$$
E_{\log }^{n}\left(U_{\mathbb{C}}\right) \otimes D_{\widetilde{X}_{\mathbb{C}} / D_{\mathbb{C} \infty}}^{m} \stackrel{\wedge}{\rightarrow} D_{\widetilde{X}_{\mathbb{C}} / D_{\mathbb{C} \infty}}^{m+n}
$$

given by $\varphi \wedge T(\omega)=T(\omega \wedge \varphi)$. This pairing turns

$$
D_{\widetilde{X}_{\mathbb{C}} / D_{\mathbb{C} \infty}}=\left(D_{\widetilde{X}_{\mathbb{C}} / D_{\mathbb{C} \infty}, \mathbb{R}}^{*}, \mathrm{~d}\right)
$$

into a Dolbeault module over the Dolbeault algebra $E_{\log }(U)=\left(E_{\log , \mathbb{R}}^{*}(U), \mathrm{d}\right)$. An application of Proposition 5.18 now shows that $\mathcal{D}_{\text {cur }, X}$ becomes a $\mathcal{D}_{\log }$-module over $\mathcal{D}_{\log , X}$.

\section{Functorial properties}

The following result is a direct consequence of the fact that the pull-back of a flat differential form is again flat.

Proposition 6.33. Let $f: X \rightarrow Y$ be a proper morphism of equidimensional schemes in $C$ of relative dimension $e$. Then, the push-forward of currents induces a covariant $f$-morphism of $\mathcal{D}_{\log }$-complexes

$$
f_{\#}: f_{*} \mathcal{D}_{\text {cur }, X} \rightarrow \mathcal{D}_{\text {cur }, Y}(-e)[-2 e] .
$$

Moreover, if $f^{\#}$ denotes the pull-back of differential forms, and $\bullet_{X}$ (respectively $\bullet_{Y}$ ) is the pairing between $\mathcal{D}_{\log , X}$ and $\mathcal{D}_{\text {cur }, X}$ (respectively $\mathcal{D}_{\log , Y}$ and $\mathcal{D}_{\text {cur }, Y}$ ), then $\left(f^{\#}, f_{\#}, f_{\#}, \bullet X, \bullet Y\right)$ is a projection five-tuple.

\section{Covariant $\mathcal{D}_{\log }$-arithmetic Chow groups}

Let $A$ be an arithmetic ring, and $X$ an arithmetic variety over $A$ such that $X_{\mathbb{R}}$ is equidimensional. Then, the pair $\left(X, \mathcal{D}_{\text {cur }}\right)$ is a $\mathcal{D}_{\text {log-arithmetic variety. Therefore, we }}$ can apply the results of $\S 4$; in particular, we can define the arithmetic Chow groups $\widehat{\mathrm{CH}}^{*}\left(X, \mathcal{D}_{\text {cur }}\right)$.

The main properties of these groups are summarized in the subsequent theorem, which is a consequence of $\S 4$ and the properties of currents discussed above.

Remark 6.34. Note that, when $X(\mathbb{C})$ is not compact, the cohomology groups of the complex $\mathcal{D}_{\text {cur }, X}$ are not the Deligne-Beilinson cohomology groups of $X$. Nevertheless, it is possible to prove that the complex $\mathcal{D}_{\text {cur }, X}$ satisfies the weak purity property. This fact is reflected by the exact sequences given in the next theorem. 
Theorem 6.35. With the above notation, we have the following statements.

(i) There is an exact sequence

$$
\mathrm{CH}^{p-1, p}(X) \stackrel{\rho}{\rightarrow} \widetilde{\mathcal{D}}_{\text {cur }}^{2 p-1}(X, p) \stackrel{\text { a }}{\rightarrow} \widehat{\mathrm{CH}}^{p}\left(X, \mathcal{D}_{\text {cur }}\right) \stackrel{\zeta}{\rightarrow} \mathrm{CH}^{p}(X) \rightarrow 0 .
$$

Moreover, if $X(\mathbb{C})$ is projective, then there is an exact sequence

$$
\begin{aligned}
& \mathrm{CH}^{p-1, p}(X) \stackrel{\rho}{\rightarrow} H_{\mathcal{D}}^{2 p-1}\left(X_{\mathbb{R}}, \mathbb{R}(p)\right) \stackrel{\mathrm{a}}{\rightarrow} \widehat{\mathrm{CH}}^{p}\left(X, \mathcal{D}_{\text {cur }}\right) \\
& \stackrel{(\zeta,-\omega)}{\longrightarrow} \mathrm{CH}^{p}(X) \oplus \mathrm{ZD}_{\text {cur }}^{2 p}(X, p) \stackrel{\text { cl }+h}{\longrightarrow} H_{\mathcal{D}}^{2 p}\left(X_{\mathbb{R}}, \mathbb{R}(p)\right) \rightarrow 0
\end{aligned}
$$

(ii) For any arithmetic variety $X$ over $A$ such that $X_{\mathbb{R}}$ is equidimensional, there is a covariant morphism of $\mathcal{D}_{\log }$-arithmetic varieties

$$
\left(X, \mathcal{D}_{\log , X}\right) \rightarrow\left(X, \mathcal{D}_{\text {cur }, X}\right),
$$

which induces a morphism of arithmetic Chow groups

$$
\widehat{\mathrm{CH}}^{p}\left(X, \mathcal{D}_{\log }\right) \rightarrow \widehat{\mathrm{CH}}^{p}\left(X, \mathcal{D}_{\text {cur }}\right) .
$$

When $X(\mathbb{C})$ is compact this morphism is injective. Moreover, if $X(\mathbb{C})$ has dimension zero, this morphism is an isomorphism.

(iii) For any proper morphism $f: X \rightarrow Y$ of arithmetic varieties over $A$ of relative dimension $e$, there is a covariant morphism of $\mathcal{D}_{\log }$-arithmetic varieties of relative dimension $e$

$$
\left(X, \mathcal{D}_{\text {cur }, X}\right) \rightarrow\left(Y, \mathcal{D}_{\text {cur }, Y}\right),
$$

which induces a morphism of arithmetic Chow groups

$$
f_{*}: \widehat{\mathrm{CH}}^{p}\left(X, \mathcal{D}_{\text {cur }}\right) \rightarrow \widehat{\mathrm{CH}}^{p-e}\left(Y, \mathcal{D}_{\text {cur }}\right) .
$$

If $g: Y \rightarrow Z$ is another such morphism, the equality $(g \circ f)_{*}=g_{*} \circ f_{*}$ holds. Moreover, if $f_{\mathbb{R}}: X_{\mathbb{R}} \rightarrow Y_{\mathbb{R}}$ is a smooth proper morphism of proper varieties, then $f_{*}$ is compatible with the direct image of contravariant arithmetic Chow groups.

(iv) The group $\widehat{\mathrm{CH}}^{*}\left(X, \mathcal{D}_{\text {cur }}\right)$ is a module over $\widehat{\mathrm{CH}}^{*}\left(X, \mathcal{D}_{\log }\right)$ with an associative action. Moreover, this action satisfies the projection formula for proper morphisms.

Canonical class of a cycle

An interesting property of covariant arithmetic Chow groups is that any algebraic cycle has its canonical class. One may think of the contravariant arithmetic Chow groups as the operational Chow groups where the characteristic classes of vector bundles live, and the covariant arithmetic Chow groups as the groups where the algebraic cycles live. 
If $y$ is a $p$-codimensional cycle with $Y=\operatorname{supp} y$, and $U=X \backslash Y$, the pair $\left(\delta_{y}, 0\right)$ represents the class of the cycle $y$ in the group

$$
H_{\mathcal{D}_{\text {cur }}, Y}^{2 p}(X, p)=H^{2 p}\left(\mathcal{D}_{\text {cur }}(X, p), \mathcal{D}_{\text {cur }}(U, p)\right) .
$$

Therefore, it is a Green object for $y$.

Definition 6.37. Let $X$ be an equidimensional arithmetic variety over $A$, and $y$ a $p$-codimensional cycle on $X$. Then, we denote by $\widehat{y} \in \widehat{\mathrm{CH}}^{p}\left(X, \mathcal{D}_{\text {cur }}\right)$ the class of the arithmetic cycle $\left(y,\left(\delta_{y}, 0\right)\right)$, i.e.

$$
\widehat{y}=\left[y,\left(\delta_{y}, 0\right)\right] \in \widehat{\mathrm{CH}}^{p}\left(X, \mathcal{D}_{\text {cur }}\right) .
$$

\subsection{Height of a cycle}

Let $K$ be a number field, $\mathcal{O}_{K}$ its ring of integers, and $S=\operatorname{Spec}\left(\mathcal{O}_{K}\right)$. Let $X$ be a $d-$ dimensional projective arithmetic variety over $\mathcal{O}_{K}$ with structural morphism $\pi: X \rightarrow S$. Since $S_{\infty}$ consists of a finite number of points, we have $E_{\log , \mathbb{R}}^{*}\left(S_{\mathbb{C}}\right)=D_{S_{\mathbb{C}}, \mathbb{R}}^{*}$. Therefore, we have

$$
\widehat{\mathrm{CH}}^{*}\left(S, \mathcal{D}_{\text {cur }}\right)=\widehat{\mathrm{CH}}^{*}\left(S, \mathcal{D}_{\log }\right) \cong \widehat{\mathrm{CH}}^{*}(S),
$$

where the isomorphism is provided by the map $\Psi$ given in Theorem 6.23.

The key ingredient in the construction of the height of a cycle in [10] is a biadditive pairing, the so-called height pairing,

$$
(\cdot \mid \cdot): \widehat{\mathrm{CH}}^{p}(X) \otimes \mathrm{Z}^{q}(X) \rightarrow \widehat{\mathrm{CH}}^{p+q-d}(S)_{\mathbb{Q}} \cdot
$$

We refer the reader to [10] for more details about this pairing. Here, we want to interpret the height pairing (6.37) in terms of the contravariant and covariant arithmetic Chow groups developed in this section.

Definition 6.39. With the above notation and conventions, we define for $\alpha \in$ $\widehat{\mathrm{CH}}^{p}\left(X, \mathcal{D}_{\log }\right)$ and $z \in \mathrm{Z}^{q}(X)$

$$
(\alpha \mid z)=\pi_{*}(\alpha \cdot \widehat{z}) \in \widehat{\mathrm{CH}}^{p+q-d}\left(S, D_{\log }\right)_{\mathbb{Q}} \cdot
$$

If $p+q=d+1$, we call the real number

$$
\operatorname{ht}_{\alpha}(z)=\widehat{\operatorname{deg}}(\alpha \mid z)
$$

the height of $z$ with respect to $\alpha$.

Proposition 6.41. There is a commutative diagram

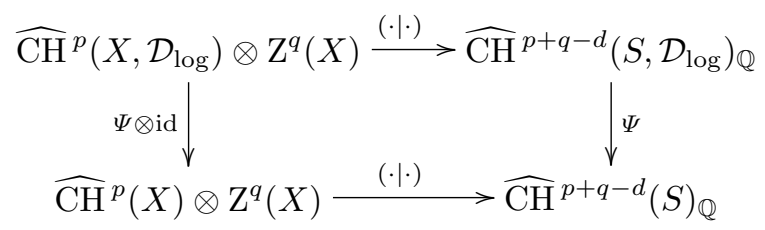

where the map $\Psi$ is given by Theorem 6.23, and the horizontal maps are provided by the pairings (6.39) and (6.37), respectively. 
Proof. We have to show that the height pairing given in Definition 6.39 translates into the height pairing of $[\mathbf{1 0}]$ using the isomorphism $\Psi$; in particular, we will see that our formula (6.39) translates into the formulae (2.3.1) and (2.3.2) therein.

If $p+q<d$, we have $\pi_{*}(\alpha \cdot \widehat{z})=0$, since $X$ has dimension $d$ over $S$. This trivially proves the claimed commutativity.

In order to treat the case $p+q=d$, we choose a representative $\left(y, \mathfrak{g}_{y}\right)$ of $\alpha$ such that $y_{K}$ intersects $z_{K}$ properly and such that $\mathfrak{g}_{y}$ is a Green object for the cycle $y$. By the $\widehat{\mathrm{CH}}^{*}\left(X, \mathcal{D}_{\log }\right)$-module structure of $\widehat{\mathrm{CH}}^{*}\left(X, \mathcal{D}_{\text {cur }}\right)$, we now compute

$$
\left(y, \mathfrak{g}_{y}\right) \cdot\left(z,\left(\delta_{z}, 0\right)\right)=\left(\left(y_{K} \cdot z_{K}, \mathfrak{g}_{y} *\left(\delta_{z}, 0\right)\right),[y \cdot z]_{\text {fin }}\right) \in \widehat{\mathrm{Z}}^{p+q}\left(X_{K}, \mathcal{D}_{\text {cur }}\right)_{\mathbb{Q}} \oplus \mathrm{CH}_{\text {fin }}^{p+q}(X)_{\mathbb{Q}},
$$

which has class $\alpha \cdot \widehat{z}$ in $\widehat{\mathrm{CH}}^{p+q}\left(X, \mathcal{D}_{\text {cur }}\right)_{\mathbb{Q}}$. Therefore, we obtain in this case

$$
(\alpha \mid z)=\pi_{*}(\alpha \cdot \widehat{z})=\left[\pi_{*}\left(y_{K} \cdot z_{K}\right),(0,0)\right] \in \widehat{\mathrm{CH}}^{0}\left(S, \mathcal{D}_{\text {cur }}\right)_{\mathbb{Q}} \cong \mathrm{CH}^{0}(S)_{\mathbb{Q}} .
$$

Taking into account formula (2.3.1) of [10], this immediately implies

$$
\Psi(\alpha \mid z)=\left[\pi_{*}\left(y_{K} \cdot z_{K}\right), 0\right]=(\Psi(\alpha) \mid z),
$$

which is the claimed commutativity.

In order to treat the case $p+q=d+1$, we let $\mathfrak{g}_{y}=\left(\omega_{y}, \widetilde{g_{y}}\right)$. Since $y_{K} \cap z_{K}=\emptyset$, we find that $g_{y} \wedge \delta_{z}$ is a well-defined closed current in $\mathcal{D}_{\text {cur }}^{2 d+1}(X, d+1)$. Using the explicit description of the $*$-product, we obtain

$$
\mathfrak{g}_{y} *\left(\delta_{z}, 0\right)=\left(\omega_{y} \wedge \delta_{z}, \widetilde{g_{y} \wedge \delta_{z}}\right)
$$

which implies

$$
\left.(\alpha \mid z)=\pi_{*}(\alpha \cdot \widehat{z})=\left[\pi_{*}\left([y \cdot z]_{\text {fin }}\right),\left(0, \pi_{\#} \widehat{\left(g_{y} \wedge\right.} \delta_{z}\right)\right)\right] \in \widehat{\mathrm{CH}}^{1}\left(S, \mathcal{D}_{\text {cur }}\right)_{\mathbb{Q}}=\widehat{\mathrm{CH}}^{1}\left(S, \mathcal{D}_{\log }\right)_{\mathbb{Q}} .
$$

By our normalization of the current associated to a cycle, we observe

$$
\pi_{\#}\left(g_{y} \wedge \delta_{z}\right)=\frac{1}{(2 \pi \mathrm{i})^{d-q}} \int_{z_{\infty}} g_{y}
$$

this shows

$$
\Psi(\alpha \mid z)=\left[\pi_{*}\left([y \cdot z]_{\mathrm{fin}}\right), \frac{2}{(2 \pi \mathrm{i})^{d-q}} \int_{z_{\infty}} g_{y}\right] .
$$

On the other hand, since $\Psi(\alpha)=\left[y, 2(2 \pi \mathrm{i})^{d-p+1}\left[g_{y}\right]_{X}\right]$, using formula $(2.3 .2)$ of $[\mathbf{1 0}]$ and respecting our definition of the current $\left[g_{y}\right]_{X}$, we compute

$$
\begin{aligned}
(\Psi(\alpha) \mid z) & =\left[\pi_{*}\left([y \cdot z]_{\mathrm{fin}}\right), \frac{1}{(2 \pi \mathrm{i})^{d}} \int_{z_{\infty}} 2(2 \pi \mathrm{i})^{d-p+1} g_{y}\right] \\
& =\left[\pi_{*}\left([y \cdot z]_{\mathrm{fin}}\right), \frac{2}{(2 \pi \mathrm{i})^{p-1}} \int_{z_{\infty}} g_{y}\right]
\end{aligned}
$$

which proves the claimed commutativity in the case $p+q=d+1$. 
We recall that there is an alternative formula for the height pairing in $[\mathbf{1 0}]$ given in terms of a choice of a Green object for the cycle $z$. Since this formula is useful for explicit computations, we describe it with the notation and normalizations used in this paper.

Lemma 6.42. Let $\alpha \in \widehat{\mathrm{CH}}^{p}\left(X, \mathcal{D}_{\log }\right)$ and $z \in \mathrm{Z}^{q}(X)$. Then, the height pairing $(\alpha \mid z)$ is given by the formula

$$
(\alpha \mid z)=\pi_{*}\left(\alpha \cdot\left[z, \mathfrak{g}_{z}\right]\right)+\mathrm{a}\left(\left[g_{z}\right]_{X}(\omega(\alpha))\right),
$$

where $\mathfrak{g}_{z}=\left(\omega_{z}, \tilde{g}_{z}\right) \in \widehat{H}_{\mathcal{D}_{\text {log }}, \mathcal{Z}^{q}}^{2 q}(X, q)$ is an arbitrarily chosen Green object for the class of $z$ and, by abuse of notation, the quantity $\left[g_{z}\right]_{X}(\omega(\alpha))$ is the real number given by

$$
\left[g_{z}\right]_{X}(\omega(\alpha))= \begin{cases}{\left[g_{z}\right]_{X}(\omega(\alpha)),} & \text { if } p+q=d+1 \\ 0, & \text { if } p+q \neq d+1\end{cases}
$$

Proof. Using Theorem 6.35 (i), we note that the element

$$
\left[z, \mathfrak{g}_{z}\right]=\left[z,\left(\omega_{z}, \widetilde{g}_{z}\right)\right] \in \widehat{\mathrm{CH}}^{q}\left(X, \mathcal{D}_{\log }\right)
$$

maps to the element

$$
\left[z,\left(\left[\omega_{z}\right], \widetilde{\left[g_{z}\right]_{X}}\right)\right] \in \widehat{\mathrm{CH}}^{q}\left(X, \mathcal{D}_{\text {cur }}\right) .
$$

By means of the $\widehat{\mathrm{CH}}^{*}\left(X, \mathcal{D}_{\log }\right)$-module structure of $\widehat{\mathrm{CH}}^{*}\left(X, \mathcal{D}_{\text {cur }}\right)$, we now compute

$$
\begin{aligned}
\pi_{*}\left(\alpha \cdot\left[z, \mathfrak{g}_{z}\right]\right)+\mathrm{a}\left(\left[g_{z}\right]_{X}(\omega(\alpha))\right) & =\pi_{*}\left(\alpha \cdot\left[z,\left(\left[\omega_{z}\right], \widetilde{\left[g_{z}\right]_{X}}\right)\right]\right)+\mathrm{a}\left(\pi_{\#}\left(\left[\omega\left(\widetilde{\alpha) \wedge g_{z}}\right]_{X}\right)\right)\right. \\
& =\pi_{*}\left(\alpha \cdot\left[z,\left(\left[\omega_{z}\right], \widetilde{\left[g_{z}\right]_{X}}\right)\right]\right)+\pi_{*}\left(\mathrm{a}\left(\left[\omega\left(\widetilde{\alpha) \wedge g_{z}}\right]_{X}\right)\right)\right. \\
& =\pi_{*}\left(\alpha \cdot\left[z,\left(\left[\omega_{z}\right], \widetilde{\left[g_{z}\right]_{X}}\right)\right]\right)+\pi_{*}\left(\widetilde{\alpha} \cdot \mathrm{a}\left(\widetilde{\left[g_{z}\right]_{X}}\right)\right) \\
& =\pi_{*}\left(\alpha \cdot\left(\left[z,\left(\left[\omega_{z}\right], \widetilde{\left[g_{z}\right]_{X}}\right)\right]+\mathrm{a}\left(\widetilde{\left[g_{z}\right]_{X}}\right)\right)\right) .
\end{aligned}
$$

Putting $\gamma=\left[g_{z}\right]_{X}$ and observing

$$
\begin{aligned}
\mathrm{a}\left(\widetilde{\left[g_{z}\right]_{X}}\right) & =\mathrm{a}(\widetilde{\gamma})=\left[0,\left(-\mathrm{d}_{\mathcal{D}} \gamma,-\widetilde{\gamma}\right)\right] \\
& =\left[0,\left(-\left[\omega_{z}\right]+\delta_{z},-\widetilde{\left[g_{z}\right]_{X}}\right)\right] \\
& =-\left[z,\left(\left[\omega_{z}\right], \widetilde{\left[g_{z}\right]_{X}}\right)\right]+\widehat{z}
\end{aligned}
$$

we find

$$
\pi_{*}\left(\alpha \cdot\left[z, \mathfrak{g}_{z}\right]\right)+\mathrm{a}\left(\left[g_{z}\right]_{X}(\omega(\alpha))\right)=\pi_{*}(\alpha \cdot \widehat{z})=(\alpha \mid z)
$$

\section{Arithmetic Chow rings with pre-log-log forms}

\subsection{Pre-log-log forms}

\section{Notation}

Let $X$ be a complex algebraic manifold of dimension $d$ and $D$ a normal crossing divisor of $X$. Write $U=X \backslash D$, and let $j: U \rightarrow X$ be the inclusion. 
Let $V$ be an open coordinate subset of $X$ with coordinates $z_{1}, \ldots, z_{d}$; we put $r_{i}=\left|z_{i}\right|$. We say that $V$ is adapted to $D$, if the divisor $D$ is locally given by the equation $z_{1} \cdots z_{k}=$ 0 . We assume that the coordinate neighbourhood $V$ is small enough; more precisely, we will assume that all the coordinates satisfy $r_{i}<1 / \mathrm{e}^{\mathrm{e}}$, which implies that $\log 1 / r_{i}>\mathrm{e}$ and $\log \left(\log 1 / r_{i}\right)>1$.

If $f$ and $g$ are two functions with non-negative real values, we will write $f \prec g$, if there exists a constant $C>0$ such that $f(x) \leqslant C \cdot g(x)$ for all $x$ in the domain of definition under consideration.

\section{Log-log growth forms}

Definition 7.1. We say that a smooth complex function $f$ on $X \backslash D$ has log-log growth along $D$, if we have

$$
\left|f\left(z_{1}, \ldots, z_{d}\right)\right| \prec \prod_{i=1}^{k} \log \left(\log \left(1 / r_{i}\right)\right)^{M}
$$

for any coordinate subset $V$ adapted to $D$ and some positive integer $M$. The sheaf of differential forms on $X$ with log-log growth along $D$ is the subalgebra of $j_{*} \mathcal{E}_{U}^{*}$ generated, in each coordinate neighbourhood $V$ adapted to $D$, by the functions with log-log growth along $D$ and the differentials

$$
\begin{array}{ll}
\frac{\mathrm{d} z_{i}}{z_{i} \log \left(1 / r_{i}\right)}, \frac{\mathrm{d} \bar{z}_{i}}{\bar{z}_{i} \log \left(1 / r_{i}\right)}, & \text { for } i=1, \ldots, k, \\
\mathrm{~d} z_{i}, \mathrm{~d} \bar{z}_{i}, & \text { for } i=k+1, \ldots, d .
\end{array}
$$

A differential form with $\log$-log growth along $D$ will be called a $\log$-log growth form.

\section{Dolbeault algebra of pre-log-log forms}

Definition 7.3. A log-log growth form $\omega$ such that $\partial \omega, \bar{\partial} \omega$ and $\partial \bar{\partial} \omega$ are also log-log growth forms is called a pre-log-log form. The sheaf of pre-log-log forms is the subalgebra of $j_{*} \mathcal{E}_{U}^{*}$ generated by the pre-log-log forms. We will denote this complex by $\mathcal{E}_{X}^{*}\langle\langle D\rangle\rangle_{\text {pre }}$.

The sheaf $\mathcal{E}_{X}^{*}\langle\langle D\rangle\rangle_{\text {pre }}$, together with its real structure, its bigrading, and the usual differential operators $\partial, \bar{\partial}$ is easily shown to be a sheaf of Dolbeault algebras. Moreover, it is the maximal subsheaf of Dolbeault algebras of the sheaf of differential forms with log-log growth.

\section{Comparison with good forms}

We start by recalling the notion of good forms from [64].

Definition 7.4. Let $X, D, U$, and $j$ be as above. A smooth function on $X \backslash D$ has Poincaré growth along $D$, if it is bounded in a neighbourhood of each point of $D$. The sheaf of differential forms with Poincaré growth along $D$ is the subalgebra of $j_{*} \mathcal{E}_{U}^{*}$ generated 
by the functions with Poincaré growth along $D$ and the differentials

$$
\begin{array}{ll}
\frac{\mathrm{d} z_{i}}{z_{i} \log \left(1 / r_{i}\right)}, \frac{\mathrm{d} \bar{z}_{i}}{\bar{z}_{i} \log \left(1 / r_{i}\right)}, & \text { for } i=1, \ldots, k, \\
\mathrm{~d} z_{i}, \mathrm{~d} \bar{z}_{i}, & \text { for } i=k+1, \ldots, d,
\end{array}
$$

for any coordinate subset $V$ adapted to $D$. A differential form $\eta$ is said to be $g o o d$, if $\eta$ and $\mathrm{d} \eta$ have Poincaré growth.

From the definition, it is clear that a form with Poincaré growth has log-log growth, but a log-log growth form, in general, does not have Poincaré growth. In contrast, a good form does not need to be a pre-log-log form. Note however that a differential form which is good, of pure bidegree and $\partial \bar{\partial}$-closed, is a pre-log-log form. In particular, a closed form of pure bidegree and Poincaré growth is also a pre-log-log form.

\section{Inverse images}

The pre-log-log forms are functorial with respect to inverse images. More precisely, we have the following result.

Proposition 7.5. Let $f: X \rightarrow Y$ be a morphism of complex algebraic manifolds, let $D_{X}, D_{Y}$ be normal crossing divisors on $X, Y$ respectively, satisfying $f^{-1}\left(D_{Y}\right) \subseteq D_{X}$. If $\eta$ is a section of $\mathcal{E}_{Y}^{*}\left\langle\left\langle D_{Y}\right\rangle\right\rangle_{\text {pre }}$, then $f^{*} \eta$ is a section of $\mathcal{E}_{X}^{*}\left\langle\left\langle D_{X}\right\rangle\right\rangle_{\text {pre }}$.

Proof. Let $P$ be a point of $X$ and $Q=f(P)$. Let $V_{X}$ (respectively $V_{Y}$ ) be a coordinate neighbourhood of $P$ (respectively $Q$ ) adapted to $D_{X}$ (respectively $D_{Y}$ ) with coordinates $z_{1}, \ldots, z_{d}$ (respectively $\left.w_{1}, \ldots, w_{n}\right)$ such that $f\left(V_{X}\right) \subseteq V_{Y}$. Assume that $D_{X}$ has equation $z_{1} \cdots z_{k}=0$ and $D_{Y}$ has equation $w_{1} \cdots w_{l}=0$. In these coordinate neighbourhoods we can write $f=\left(f_{1}, \ldots, f_{n}\right)$. The condition $f^{-1}\left(D_{Y}\right) \subseteq D_{X}$ means that the divisor of $f_{i}$ is contained in $D_{X}$ for $i=1, \ldots, l$. Therefore, we can write

$$
f_{i}=u_{i} \prod_{j=1}^{k} z_{j}^{\alpha_{i, j}},
$$

where the $\alpha_{i, j}$ are non-negative integers and the $u_{i}$ are units.

We start by showing that the pre-image of a log-log growth form has log-log growth. If $h$ is a log-log growth function on $Y \backslash D_{Y}$, then in order to show that $h \circ f$ has also log-log growth on $X \backslash D_{X}$, one uses the inequality

$$
\log (a+b)<2(\log (a)+\log (b)) \leqslant 4 \log (a) \log (b)
$$

for $a, b \geqslant e$. Next we consider $f^{*}\left(\mathrm{~d} w_{i} /\left(w_{i} \log \left(1 /\left|w_{i}\right|\right)\right)\right)$; we have

$$
\begin{aligned}
f^{*}\left(\frac{\mathrm{d} w_{i}}{w_{i} \log \left(1 /\left|w_{i}\right|\right)}\right) & =\frac{\mathrm{d} f_{i}}{f_{i} \log \left(1 /\left|f_{i}\right|\right)} \\
& =\frac{\sum_{j=1}^{k} \alpha_{i, j} \mathrm{~d} z_{j} / z_{j}+\mathrm{d} u_{i} / u_{i}}{\sum_{j=1}^{k} \alpha_{i, j} \log \left(1 / r_{j}\right)+\log \left(1 /\left|u_{i}\right|\right)} .
\end{aligned}
$$


Since, for any $j_{0}$, the functions

$$
\frac{\log \left(1 / r_{j_{0}}\right)}{\sum_{j=1}^{k} \alpha_{i, j} \log \left(1 / r_{j}\right)+\log \left(1 / u_{i}\right)}, \quad \frac{\log \left(1 / u_{i}\right)}{\sum_{j=1}^{k} \alpha_{i, j} \log \left(1 / r_{j}\right)+\log \left(1 / u_{i}\right)}
$$

have $\log$-log growth, we obtain that $f^{*}\left(\mathrm{~d} w_{i} /\left(w_{i} \log \left(1 /\left|w_{i}\right|\right)\right)\right)$ is a log-log growth form. The analogous result holds true for the complex conjugate form.

Finally, in order to show that the pre-image of a pre-log-log form is a pre-log-log form one uses the compatibility between inverse images and the operators $\partial$ and $\bar{\partial}$.

\section{Integrability}

Despite the fact that pre-log-log forms and good forms are not exactly the same, they share many properties. For instance the following result is the analogue of Propositions 1.1 and 1.2 in [64].

\section{Proposition 7.6.}

(i) Any log-log growth form is locally integrable.

(ii) If $\eta$ is a pre-log-log form, and $[\eta]_{X}$ is the associated current, then

$$
[\mathrm{d} \eta]_{X}=\mathrm{d}[\eta]_{X}
$$

The same holds true for the differential operators $\partial, \bar{\partial}$ and $\partial \bar{\partial}$.

Proof. Recall that $d$ is the dimension of $X$. For the first statement it is enough to show that, if $\eta$ is a $(d, d)$-form on $X$ with log-log growth along $D$ and $V$ is an open neighbourhood adapted to $D$, then we have

$$
\left|\int_{V} \eta\right|<\infty
$$

Let us denote by $\Delta_{1 / \mathrm{e}^{\mathrm{e}}}^{*} \subseteq V$ the punctured disc of radius $1 / \mathrm{e}^{\mathrm{e}}$. We now have the estimates

$$
\begin{aligned}
\left|\int_{V} \eta\right| & <C_{1} \prod_{i=1}^{k} \int_{\Delta_{1 / \mathrm{e}}^{*}}\left|\log \left(\log \left(1 / r_{i}\right)\right)^{M} \frac{\mathrm{d} z_{i} \wedge \mathrm{d} \bar{z}_{i}}{z_{i} \bar{z}_{i} \log \left(1 / r_{i}\right)^{2}}\right| \\
& <C_{2} \prod_{i=1}^{k} \int_{0}^{1 / \mathrm{e}^{\mathrm{e}}} \log \left(\log \left(1 / r_{i}\right)\right)^{M} \frac{\mathrm{d} r_{i}}{r_{i} \log \left(1 / r_{i}\right)^{2}} \\
& <C_{3} \prod_{i=1}^{k} \int_{0}^{1 / \mathrm{e}^{\mathrm{e}}} \frac{\mathrm{d} r_{i}}{r_{i} \log \left(1 / r_{i}\right)^{1+\varepsilon}}<\infty
\end{aligned}
$$

for some positive real constants $C_{1}, C_{2}, C_{3}$ and $\varepsilon$, and a positive integer $M$. The proof of the second statement is analogous to the proof of Proposition 1.2. in [64].

\subsection{Pre-log forms}

We will now define a complex which contains pre-log-log forms as well as the differential forms with logarithmic singularities introduced in $\S 5.3$. 
Log growth forms

Let $X, D, U$ and $j$ be as in the previous section.

Definition 7.7. We say that a smooth complex function $f$ on $U$ has $\log$ growth along $D$, if we have

$$
\left|f\left(z_{1}, \ldots, z_{d}\right)\right| \prec \prod_{i=1}^{k} \log \left(1 / r_{i}\right)^{M}
$$

for any coordinate subset $V$ adapted to $D$ and some positive integer $M$. The sheaf of differential forms on $X$ with $\log$ growth along $D$ is the subalgebra of $j_{*} \mathcal{E}_{V}^{*}$ generated, in each coordinate neighbourhood $V$ adapted to $D$, by the functions with log growth along $D$ and the differentials

$$
\begin{array}{ll}
\frac{\mathrm{d} z_{i}}{z_{i}}, \frac{\mathrm{d} \bar{z}_{i}}{\bar{z}_{i}}, & \text { for } i=1, \ldots, k, \\
\mathrm{~d} z_{i}, \mathrm{~d} \bar{z}_{i}, & \text { for } i=k+1, \ldots, d .
\end{array}
$$

A differential form with $\log$ growth along $D$ will be called a $\log$ growth form.

Dolbeault algebra of pre-log forms

Definition 7.9. A $\log$ growth form $\omega$ such that $\partial \omega, \bar{\partial} \omega$ and $\partial \bar{\partial} \omega$ are also log growth forms is called a pre-log form. The sheaf of pre-log forms is the subalgebra of $j_{*} \mathcal{E}_{U}^{*}$ generated by the pre-log forms. We will denote this complex by $\mathcal{E}_{X}^{*}\langle D\rangle_{\text {pre }}$.

The sheaf $\mathcal{E}_{X}^{*}\langle D\rangle_{\text {pre }}$, together with its real structure, its bigrading and the usual differential operators $\partial, \bar{\partial}$ is easily shown to be a sheaf of Dolbeault algebras. Moreover, it is the maximal subsheaf of Dolbeault algebras of the sheaf of differential forms with log growth.

\subsection{Mixed forms}

For the general situation which interests us, we need a combination of the concepts of pre-log-log and pre-log forms.

\section{Mixed growth forms}

Let $X, D, U$ and $j$ be as in the previous section. Let $D_{1}$ and $D_{2}$ be normal crossing divisors, which may have common components, and such that $D=D_{1} \cup D_{2}$. We denote by $D_{2}^{\prime}$ the union of the components of $D_{2}$ which are not contained in $D_{1}$. We will say that the open coordinate subset $V$ is adapted to $D_{1}$ and $D_{2}$, if $D_{1}$ has equation $z_{1} \cdots z_{k}=0$, $D_{2}^{\prime}$ has equation $z_{k+1} \cdots z_{l}=0$ and that $r_{i}=\left|z_{i}\right|<1 / \mathrm{e}^{\mathrm{e}}$ for $i=1, \ldots, d$.

Definition 7.10. We define the sheaf of forms with log growth along $D_{1}$ and $\log$-log growth along $D_{2}$ to be the subalgebra of $j_{*} \mathcal{E}_{U}^{*}$ generated by differential forms with $\log$ growth along $D_{1}$ and log-log growth along $D_{2}$.

A differential form with $\log$ growth along $D_{1}$ and $\log$-log growth along $D_{2}$ will be called a mixed growth form, if the divisors $D_{1}$ and $D_{2}$ are clear from the context. 
Dolbeault algebra of mixed forms

Definition 7.11. Let $X, D=D_{1} \cup D_{2}, U$ and $j$ be as before. A mixed growth form $\omega$ such that $\partial \omega, \bar{\partial} \omega$ and $\partial \bar{\partial} \omega$ are also mixed growth forms is called a mixed form. The sheaf of mixed forms is the subalgebra of $j_{*} \mathcal{E}_{U}^{*}$ generated by the mixed forms. We will denote this complex by $\mathcal{E}_{X}^{*}\left\langle D_{1}\left\langle D_{2}\right\rangle\right\rangle_{\text {pre }}$.

The sheaf $\mathcal{E}_{X}^{*}\left\langle D_{1}\left\langle D_{2}\right\rangle\right\rangle_{\text {pre }}$ together with its real structure, its bigrading and the usual differential operators $\partial, \bar{\partial}$ is easily checked to be a sheaf of Dolbeault algebras. Observe that we have by definition

$$
\mathcal{E}_{X}^{*}\left\langle D_{1}\left\langle D_{2}\right\rangle\right\rangle_{\mathrm{pre}}=\mathcal{E}_{X}^{*}\left\langle D_{1}\left\langle D_{2}^{\prime}\right\rangle\right\rangle_{\mathrm{pre}}
$$

\section{Inverse images}

We can generalize Proposition 7.5 as follows. The proof is similar to the proof of Proposition 7.5, and is therefore left to the reader.

Proposition 7.12. Let $f: X \rightarrow Y$ be a morphism of complex algebraic manifolds. Let $D_{1}, D_{2}$ and $E_{1}, E_{2}$ be normal crossing divisors on $X, Y$, respectively, such that $D_{1} \cup D_{2}$ and $E_{1} \cup E_{2}$ are also normal crossing divisors. Furthermore, assume that $f^{-1}\left(E_{1}\right) \subseteq D_{1}$ and $f^{-1}\left(E_{2}\right) \subseteq D_{1} \cup D_{2}$. If $\eta$ is a section of $\mathcal{E}_{Y}^{*}\left\langle E_{1}\left\langle E_{2}\right\rangle\right\rangle_{\text {pre }}$, then $f^{*} \eta$ is a section of $\mathcal{E}_{X}^{*}\left\langle D_{1}\left\langle D_{2}\right\rangle\right\rangle_{\text {pre }}$.

\section{Integrability}

Let $y$ be a $p$-codimensional cycle of $X, Y=\operatorname{supp} y$, and $U=X \backslash Y$. Let $\pi: \widetilde{X} \rightarrow X$ be an embedded resolution of singularities of $Y$ with normal crossing divisors $D_{Y}=\pi^{-1}(Y)$ and $\widetilde{D}=\pi^{-1}(D)$.

Lemma 7.13. Assume that $g \in \Gamma\left(\widetilde{X}, \mathcal{E}_{\widetilde{X}}^{n}\left\langle D_{Y}\langle\widetilde{D}\rangle\right\rangle_{\text {pre }}\right)$. Then, the following statements hold.

(i) If $n<2 p$, then $g$ is locally integrable on the whole of $X$. We denote by $[g]_{X}$ the current associated to $g$.

(ii) If $n<2 p-1$, then $\mathrm{d}[g]_{X}=[\mathrm{d} g]_{X}$.

Proof. Recall again that $d$ is the dimension of $X$. To prove the first statement, we have to show that for any differential form $\alpha$ of degree $2 d-n>2 d-2 p$ and any compact set $K \subseteq X$, the integral

$$
\int_{K} \alpha \wedge g
$$

is convergent. The restriction of $\pi^{*} \alpha$ to $D_{Y}$ vanishes, since the map $D_{Y} \rightarrow X$ factors through $Y$. Therefore, if $z_{1} \cdots z_{k}=0$ is a local equation for $D_{Y}, \pi^{*} \alpha$ can be decomposed as a sum of terms, each of which contains a factor $\mathrm{d} z_{i}, \mathrm{~d} \bar{z}_{i}, z_{i}$, or $\bar{z}_{i}$ for $i=1, \ldots, k$. The result now follows from the local expression describing logarithmic growth. The second statement is proven analogously. 


\section{Good Metrics}

We recall Mumford's notion of a good metric (see [64]) in the case of line bundles.

Definition 7.14. Let $X$ be a complex algebraic manifold, $D$ a normal crossing divisor, and put $U=X \backslash D$. Let $L$ be a line bundle on $X$, and $L_{\mid U}$ the restriction of $L$ to $U$. A smooth hermitian metric $h$ on $L_{\mid U}$ is said to be good along $D$, if we have for all $x \in D$, all neighbourhoods $V$ of $x$ adapted to $D$ (with coordinates $z_{1}, \ldots, z_{d}$ ), and all non-vanishing, rational sections $s$ of $L$ (writing $\|s\|^{2}=h(s, s)$ ):

(i) $\|s\|,\|s\|^{-1} \leqslant C\left|\prod_{i=1}^{k} \log \left(r_{i}\right)\right|^{N}$ for some $C>0$ and some $N \in \mathbb{N}$;

(ii) the 1-form $\partial \log \|s\|$ is good on $V$.

A line bundle $L$ equipped with a good hermitian metric will be called a good hermitian line bundle. The pair $(L, h)$ will be denoted by $\bar{L}$.

Let $X, D, U$ be as in the preceding definition, and let $\bar{L}$ be a good hermitian line bundle on $X$. For a non-vanishing, rational section $s$ of $L$, let $Y$ denote the support of $\operatorname{div}(s)$, and put $V=X \backslash Y$. Let $\pi: \widetilde{X} \rightarrow X$ be an embedded resolution of singularities of $Y$ with the property that

$$
D_{Y}=\pi^{-1}(Y), \quad \widetilde{D}=\pi^{-1}(D) \quad \text { and } \quad \pi^{-1}(Y \cup D)
$$

are normal crossing divisors.

Proposition 7.15. With the above notation we have

$$
\log \|s\| \in \Gamma\left(\widetilde{X}, \mathcal{E}_{\widetilde{X}}^{0}\left\langle D_{Y}\langle\widetilde{D}\rangle\right\rangle_{\text {pre }}\right), \quad \partial \bar{\partial} \log \|s\| \in \Gamma\left(\widetilde{X}, \mathcal{E}_{\widetilde{X}}^{1,1}\langle\langle\widetilde{D}\rangle\rangle_{\text {pre }}\right) .
$$

Proof. First we show that $\omega_{s}=\partial \bar{\partial} \log \|s\|$ is a pre-log-log form. If $s^{\prime}$ is another nonvanishing, rational section of $L$ in an open subset $V$ of $X$, we note that $\left.\omega_{s}\right|_{V}=\left.\omega_{s^{\prime}}\right|_{V}$. This shows that $\partial \bar{\partial} \log \|s\|$ gives rise to a differential form $\omega$ on $X$, which is independent of the choice of $s$. By the very definition of a good metric, $\omega$ has Poincaré growth along $D \cap V$. Since this is true for any open covering of $X, \omega$ has Poincaré growth along $D$. Therefore, it is a log-log growth form. Since $\omega$ is closed, $\partial \omega, \bar{\partial} \omega$, and $\partial \bar{\partial} \omega$ have also log-log growth. This proves that $\omega$ is a pre-log-log form along $D$.

We now show that $\log \|s\|$ is a pre-log form along $D_{Y}$ and a pre-log-log form along $\widetilde{D}$. In a neighbourhood of a point of $D_{Y} \backslash \widetilde{D}$, the function $\log \|s\|$ has logarithmic singularities along $D_{Y}$, as shown in Proposition 5.64. Therefore, $\log \|s\|$ is a pre-log form. On the other hand, in a neighbourhood of a point of $\widetilde{D} \backslash D_{Y}$, the function $\|s\|$ has log growth along $\widetilde{D}$, since $h$ is a good metric; hence, $\log \|s\|$ has log-log growth. Moreover, the forms $\partial \log \|s\|, \bar{\partial} \log \|s\|$, and $\partial \bar{\partial} \log \|s\|$ have Poincaré growth. This shows that $\log \|s\|$ is a pre-log-log form along $\widetilde{D} \backslash D_{Y}$. Finally, in a neighbourhood of a point of $D_{Y} \cap \widetilde{D}$, we can write $\log \|s\|$ as the sum of a form with logarithmic singularities and a pre-log-log form. Therefore, $\log \|s\|$ is a pre-log form there. 


\subsection{A $\mathcal{D}_{\log }$-complex with pre-log-log forms}

In this section we will construct a $\mathcal{D}_{\log }$-complex using pre-log-log forms along a fixed normal crossing divisor.

\section{Varieties with a fixed normal crossing divisor}

Let $X$ be a complex algebraic manifold of dimension $d$, and $D$ a normal crossing divisor. We will denote the pair $(X, D)$ by $\underline{X}$. If $V \subseteq X$ is an open subset, we will write $\underline{V}=(V, D \cap V)$.

In the sequel all operations have to be adapted with respect to the pair $\underline{X}$. For instance, if $Y \subseteq X$ is a closed algebraic subset and $V=X \backslash Y$, we mean by an embedded resolution of singularities of $Y$ adapted to $D$ an embedded resolution of singularities $\pi: \widetilde{X} \rightarrow X$ of $Y$ with the property that

$$
D_{Y}=\pi^{-1}(Y), \quad \widetilde{D}=\pi^{-1}(D) \quad \text { and } \quad \pi^{-1}(Y \cup D)
$$

are normal crossing divisors. Using Hironaka's theorem on the resolution of singularities [44], one can show that such an embedded resolution of singularities exists; for a more detailed description of this fact we refer to Theorem 7.29 , below.

Analogously, a normal crossing compactification of $\underline{X}$ will be a smooth compactification $\bar{X}$ such that the adherence $\bar{D}$ of $D$ and the subsets $B_{\bar{X}}=\bar{X} \backslash X$ and $B_{\bar{X}} \cup \bar{D}$ are normal crossing divisors.

\section{Pre-log along infinity}

Given a diagram of normal crossing compactifications of $\underline{X}$

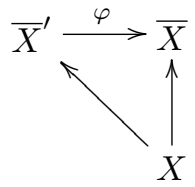

with divisors $B_{\bar{X}^{\prime}}$ and $B_{\bar{X}}$ at infinity, respectively, Proposition 7.12 gives rise to an induced morphism

$$
\varphi^{*}: \mathcal{E}_{\bar{X}}^{*}\left\langle B_{\bar{X}}\langle\bar{D}\rangle\right\rangle_{\text {pre }} \rightarrow \mathcal{E}_{\bar{X}^{\prime}}^{*}\left\langle B_{\bar{X}^{\prime}}\left\langle\bar{D}^{\prime}\right\rangle\right\rangle_{\text {pre }} .
$$

In order to have a complex which is independent of the choice of a particular compactification, as in $\S 5.3$, we take the limit over all possible compactifications. For $\underline{X}=(X, D)$ as before, we then denote

$$
E_{\text {pre }}^{*}(\underline{X})^{\circ}=\lim _{\rightarrow} \Gamma\left(\bar{X}, \mathcal{E}_{\bar{X}}^{*}\left\langle B_{\bar{X}}\langle\bar{D}\rangle\right\rangle_{\text {pre }}\right),
$$

where the limit is taken over all normal crossing compactifications $\bar{X}$ of $\underline{X}$.

The assignment which sends an open subset $U$ of $X$ to $E_{\mathrm{pre}}^{*}(\underline{U})^{\circ}$ is a presheaf in the Zariski topology; we denote by $E_{\text {pre, } \underline{X}}^{*}$ its associated sheaf. 
Definition 7.16. Let $\underline{X}=(X, D)$ be as above. Then, we define the complex $E_{\text {pre }}^{*}(\underline{X})$ of differential forms on $X$, pre-log along infinity and pre-log-log along $D$ as the complex of global sections of $E_{\mathrm{pre}, \underline{X}}^{*}$, i.e.

$$
E_{\text {pre }}^{*}(\underline{X})=\Gamma\left(X, E_{\text {pre }, \underline{X}}^{*}\right) .
$$

Remark 7.17. Since in the definition of the presheaf $E_{\mathrm{pre}}^{*}(\underline{X})^{\circ}$ we are using growth conditions it might be possible that this presheaf is already a sheaf.

$A \mathcal{D}_{\log }$-complex

Let $X$ be a smooth real variety and $D$ a normal crossing divisor defined over $\mathbb{R}$; as before, we write $\underline{X}=(X, D)$. For any $U \subseteq X$, the complex $E_{\text {pre }}^{*}(\underline{U})$ is a Dolbeault algebra with respect to the wedge product.

Definition 7.18. For any Zariski open subset $U \subseteq X$, we put

$$
\mathcal{D}_{\text {pre }, \underline{X}}^{*}(U, p)=\left(\mathcal{D}_{\text {pre }, \underline{X}}^{*}(U, p), \mathrm{d}_{\mathcal{D}}\right)=\left(\mathcal{D}^{*}\left(E_{\text {pre }}\left(\underline{U}_{\mathbb{C}}\right), p\right)^{\sigma}, \mathrm{d}_{\mathcal{D}}\right),
$$

where $\sigma$ is as in Notation 5.65.

Theorem 7.19. The complex $\mathcal{D}_{\text {pre }, \underline{X}}$ is a $\mathcal{D}_{\log }$-complex on $X$. Moreover, it is a pseudoassociative and commutative $\mathcal{D}_{\text {log-algebra. }}$

Proof. As in Proposition 5.33, we obtain that the presheaf $\mathcal{D}_{\text {pre }, X}^{n}(\cdot, p)$ satisfies the Mayer-Vietoris principle for any pair of integers $p, n$. Therefore, it is a totally acyclic sheaf. Clearly, there are morphisms of sheaves of algebras

$$
\mathcal{D}_{\log , X}^{*} \rightarrow \mathcal{D}_{\text {pre }, \underline{X}}^{*}
$$

The claim now follows.

Remark 7.20. Because of technical reasons we are not able to prove or disprove a filtered Poincaré lemma for the complex $E_{\text {pre, } \underline{X}}^{*}$. Therefore, we do not know the exact cohomology of the complex $\mathcal{D}_{\text {pre, }, \underline{X}}^{*}$. Nevertheless, if $X$ is projective, we note that the composition

$$
\mathcal{D}^{*}\left(E_{X}, p\right) \rightarrow \mathcal{D}^{*}\left(E_{X}\langle\langle D\rangle\rangle_{\text {pre }}, p\right) \rightarrow \mathcal{D}^{*}\left(D_{X}, p\right)
$$

is a quasi-isomorphism. Therefore, the least we can say is that the cohomology of $\mathcal{D}^{*}\left(E_{X}\langle\langle D\rangle\rangle_{\text {pre }}, p\right)$ has the usual real Deligne-Beilinson cohomology as a direct summand. As has been mentioned before, this problem can be solved by imposing growth conditions on all derivatives of the differential forms under consideration.

\subsection{Properties of Green objects with values in $\mathcal{D}_{\text {pre }}$}

We start by noting that Theorem 7.19 together with $\S 3.2$ provides us with a theory of Green objects with values in $\mathcal{D}_{\text {pre }}$. 
Mixed forms representing the class of a cycle

In the case of pre-log-log forms the analogue of Proposition 5.58 is less precise, since we do not know the cohomology of the complex of pre-log-log forms.

Proposition 7.21. Let $X$ be a smooth real variety, and $y$ a $p$-codimensional cycle on $X$ with support $Y$. Let $(\omega, g)$ be a cycle in

$$
s^{2 p}\left(\mathcal{D}_{\text {pre }, \underline{X}}(X, p) \rightarrow \mathcal{D}_{\text {pre }, \underline{X}}(X \backslash Y, p)\right) .
$$

Then, we have the following statements.

(i) If the class of the cycle $(\omega, g)$ in $H_{\mathcal{D}_{\text {pre }}, Y}^{2 p}(X, p)$ is equal to the class of $y$, then

$$
-2 \partial \bar{\partial}[g]_{X}=[\omega]-\delta_{y} .
$$

(ii) Assume that $y=\sum_{j} n_{j} Y_{j}$ with irreducible subvarieties $Y_{j}$ and certain multiplicities $n_{j}$. If the cycle $(\omega, g)$ represents the class of $y$, then the equality

$$
-\lim _{\varepsilon \rightarrow 0} \int_{\partial B_{\varepsilon}(Y)} \alpha \mathrm{d}^{\mathrm{c}} g=\frac{(2 \pi \mathrm{i})^{p-1}}{2} \sum_{j} n_{j} \int_{Y_{j}} \alpha
$$

holds for any differential form $\alpha$; here $B_{\varepsilon}(Y)$ is an $\varepsilon$-neighbourhood of $Y$ such that the orientation of $\partial B_{\varepsilon}(Y)$ is induced from the orientation of $B_{\varepsilon}(Y)$.

(iii) Let $g_{y}$ be a differential form on $X \backslash(Y \cup D)$ such that there exists an embedded resolution of singularities $\pi: \widetilde{X} \rightarrow X$ of $Y$ in $\underline{X}$ with $D_{Y}=\pi^{-1}(Y)$ and $\widetilde{D}=\pi^{-1}(D)$, such that in any coordinate neighbourhood adapted to $D_{Y}$, we have

$$
\pi^{*}\left(g_{y}\right)=\sum_{i=1}^{k} \alpha_{i} \log \left(1 / r_{i}\right)+\beta,
$$

where $\alpha_{i}$ are smooth forms on $\tilde{X}$ and $\beta$ is the pull-back of a pre-log-log form on $\underline{X}$. If the pair $\left(\omega_{y}, g_{y}\right)=\left(-2 \partial \bar{\partial} g_{y}, g_{y}\right)$ is a cycle in

$$
s^{2 p}\left(\mathcal{D}_{\text {pre }, \underline{X}}(X, p) \rightarrow \mathcal{D}_{\text {pre }, \underline{X}}(X \backslash Y, p)\right)
$$

and $g_{y}$ satisfies one of the equations $(7.21)$ or $(7.22)$, then the pair $\left(\omega_{y}, \widetilde{g}_{y}\right)$ is a pre-log-log Green object for the cycle $y$.

Proof. (i) Let $\left(\omega^{\prime}, g^{\prime}\right)$ be a cycle representing the class of $y$ in $H_{\mathcal{D}, Y}^{2 p}(X, \mathbb{R}(p))$. By Proposition 5.58 , we then have

$$
-2 \partial \bar{\partial}\left[g^{\prime}\right]_{X}=\left[\omega^{\prime}\right]-\delta_{y} .
$$

By our assumption, the pair $(\omega, g)$ and the image of $\left(\omega^{\prime}, g^{\prime}\right)$ represent the same class in $H_{\mathcal{D}_{\text {pre }}, Y}^{2 p}(X, p)$. Therefore, there are elements $a \in \mathcal{D}_{\text {pre }, \underline{X}}^{2 p-1}(X, p)$ and $b \in \mathcal{D}_{\text {pre }, \underline{X}}^{2 p-2}(X \backslash Y, p)$ such that

$$
\left(\mathrm{d}_{\mathcal{D}} a, a-\mathrm{d}_{\mathcal{D}} b\right)=(\omega, g)-\left(\omega^{\prime}, g^{\prime}\right) .
$$

The result now follows as in Proposition 5.58 (i) using Proposition 7.6 and Lemma 7.13.

(ii) The second statement follows as in Proposition 5.58 (ii).

(iii) Since pre-log-log forms have no residues we may deduce this statement from Proposition 5.58. 
The first Chern form of a line bundle

Let $X$ be a quasi-projective smooth real variety, $D$ a normal crossing divisor defined over $\mathbb{R}$, and $\bar{L}=(L, h)$ a hermitian line bundle over $X$, where the hermitian metric $h$ on $L \otimes \mathbb{C}$ is good along $D$ and satisfies $F_{\infty}^{*} h=h$. If $s$ is a non-vanishing, rational section of $L$, we put $\|s\|^{2}=h(s, s), y=\operatorname{div}(s), Y=\operatorname{supp} y, U=X \backslash Y$, and (see $\S 5.6$ )

$$
\begin{aligned}
g_{s} & =-\frac{1}{2} \log \left(\|s\|^{2}\right), \\
\omega_{s} & =-2 \partial \bar{\partial} g_{s} .
\end{aligned}
$$

Moreover, we put $\underline{X}=(X, D)$, and $\underline{U}=(U, U \cap D)$. We call

$$
\mathrm{c}_{1}(\bar{L})=\omega_{s}=-2 \partial \bar{\partial} g_{s}=\partial \bar{\partial} \log \left(\|s\|^{2}\right)
$$

the first Chern form of $\bar{L}$.

Proposition 7.25. With the above assumptions the following statements hold. The form $\omega_{s}$ belongs to $\mathcal{D}_{\text {pre }, \underline{X}}^{2}(X, 1)$, the form $g_{s}$ belongs to $\mathcal{D}_{\text {pre }, \underline{X}}^{1}(U, 1)$. The pair $\left(\omega_{s}, g_{s}\right)$ is a cycle of the simple complex

$$
s^{2}\left(\mathcal{D}_{\text {pre }, \underline{X}}(X, 1) \rightarrow \mathcal{D}_{\text {pre }, \underline{X}}(U, 1)\right) .
$$

Moreover, this pair represents the class of $\operatorname{div}(s)$ in the cohomology group $H_{\mathcal{D}_{\text {pre }}, Y}^{2}(X, 1)$.

Proof. We first show that the form $\omega_{s}$ belongs to $\mathcal{D}_{\text {pre }, \underline{X}}^{2}(X, 1)$. By Proposition 7.15, the form $\omega_{s}$ is a pre-log-log form. The invariance of $h$ with respect to $F_{\infty}$ now shows that $\omega_{s}$ belongs to $\mathcal{D}_{\text {pre }, \underline{X}}^{2}(X, 1)$. Analogously, again using Proposition 7.15 , we obtain that $g_{s} \in \mathcal{D}_{\text {pre }, \underline{X}}^{1}(U, 1)$.

Let now $h^{\prime}$ be a hermitian metric on $L$, which is invariant under $F_{\infty}$ and smooth on the whole of $X$. Let us write

$$
\begin{aligned}
g_{s}^{\prime} & =-\frac{1}{2} \log \left(\|s\|^{\prime 2}\right), \\
\omega_{s}^{\prime} & =-2 \partial \bar{\partial} g_{s}^{\prime} .
\end{aligned}
$$

By Proposition 5.64 , the class of $\operatorname{div}(s)$ in $H_{\mathcal{D}_{\log }, Y}^{2}(X, 1)$ is represented by the pair $\left(\omega_{s}^{\prime}, g_{s}^{\prime}\right)$. On the other hand, we have

$$
g_{s}-g_{s}^{\prime} \in \mathcal{D}_{\text {pre }, \underline{X}}^{1}(X, 1)
$$

and therefore

$$
\mathrm{d}_{\mathcal{D}}\left(g_{s}-g_{s}^{\prime}, 0\right)=\left(\omega_{s}, g_{s}\right)-\left(\omega_{s}^{\prime}, g_{s}^{\prime}\right) .
$$

Thus both pairs represent the same cohomology class.

Formulae for the *-product

The formulae of Proposition 6.10 still hold true in this more general context. Moreover, if we consider embedded resolutions adapted to $\underline{X}$, it is not difficult to show that Theorem 6.12 also remains true. 
Inverse images

Proposition 7.26. Let $f: X \rightarrow Y$ be a morphism of smooth real varieties, let $D_{X}$, $D_{Y}$ be normal crossing divisors on $X, Y$ respectively, satisfying $f^{-1}\left(D_{Y}\right) \subseteq D_{X}$. Put $\underline{X}=\left(X, D_{X}\right)$ and $\underline{Y}=\left(Y, D_{Y}\right)$. Then, there exists a contravariant $f$-morphism

$$
f^{\#}: \mathcal{D}_{\text {pre }, \underline{Y}} \rightarrow f_{*} \mathcal{D}_{\text {pre }, \underline{X}} .
$$

Proof. By Proposition 7.12, the pull-back of differential forms induces a morphism of the corresponding Dolbeault algebras of mixed forms. This morphism is compatible with the involution $\sigma$. Thus, this morphism gives rise to an induced morphism between the corresponding Deligne algebras.

\section{Push-forward}

We will only state the most basic property concerning direct images, which is necessary to define arithmetic degrees. Note however that we expect log-log forms to be useful in the study of non smooth, proper, surjective morphisms. By Proposition 7.6, we have the following proposition.

Proposition 7.27. Let $\underline{X}=(X, D)$ be a proper, smooth real variety with fixed normal crossing divisor $D$. Let $f: X \rightarrow \operatorname{Spec}(\mathbb{R})$ denote the structural morphism. Then, there exists a covariant $f$-morphism

$$
f_{\#}: f_{*} \mathcal{D}_{\text {pre }, \underline{X}} \rightarrow \mathcal{D}_{\log , \operatorname{Spec}(\mathbb{R})}
$$

In particular, if $X$ has dimension $d$, we obtain a well-defined morphism

$$
f_{\#}: \widehat{H}_{\mathcal{D}_{\text {pre }}, \mathcal{Z}^{d+1}}^{2 d+2}(X, d) \rightarrow \widehat{H}_{\mathcal{D}_{\text {log }}, \mathcal{Z}^{1}}^{2}(\operatorname{Spec}(\mathbb{R}), 1)=\mathbb{R} .
$$

Note that, by dimension reasons, we have $\mathcal{Z}^{d+1}=\emptyset$, and

$$
\widehat{H}_{\mathcal{D}_{\text {pre }}, \mathcal{Z}^{d+1}}^{2 d+2}(X, d)=H^{2 d+1}\left(\mathcal{D}_{\text {pre }, \underline{X}}(X, d+1)\right) .
$$

Thus, every element of $\widehat{H}_{\mathcal{D}_{\text {pre }}, \mathcal{Z}^{d+1}}^{2 d+2}(X, d)$ is represented by a pair $\mathfrak{g}=(0, \widetilde{g})$. The morphism $f_{\#}$ mentioned above, is then given by

$$
\mathfrak{g}=(0, \widetilde{g}) \mapsto\left(0, \frac{1}{(2 \pi \mathrm{i})^{d}} \int_{X} g\right)
$$

Notation 7.28. For $\mathfrak{g}=(0, \widetilde{g}) \in \widehat{H}_{\mathcal{D}_{\text {pre }}, \mathcal{Z}^{d+1}}^{2 d+2}(X, d)$, we will write

$$
\frac{1}{(2 \pi \mathrm{i})^{d}} \int_{X} \mathfrak{g}=\frac{1}{(2 \pi \mathrm{i})^{d}} \int_{X} g .
$$




\subsection{Push-forward of a $*$-product}

\section{Embedded resolution of singularities}

We now come to a more detailed description of embedded resolutions of singularities of closed algebraic subsets adapted to a fixed normal crossing divisor, which has been announced in $\S 7.4$. This description is a consequence of the following precise version of Hironaka's theorem on the resolution of singularities, which is contained in [44]. These considerations provide an important tool for explicit computations of $*$-products.

Theorem 7.29 (Hironaka). Let $X$ be a smooth variety over a field of characteristic zero. Let $D$ be a normal crossing divisor of $X$, and $Y$ an irreducible reduced subscheme of $X$. Then, there is a finite sequence of smooth varieties $\widetilde{X}_{k}$ and subschemes $D_{k}, Y_{k}$, $W_{k}(k=0, \ldots, N)$ satisfying

(i) $\widetilde{X}_{0}=X, D_{0}=D, Y_{0}=Y$;

(ii) for $k=0, \ldots, N$, the subscheme $W_{k}$ is contained in $Y_{k}$, the subscheme $Y_{k}$ is normally flat along $W_{k}$, the subscheme $D_{k}$ is a normal crossing divisor, and the pair $\left(D_{k}, W_{k}\right)$ has only normal crossings;

(iii) $\widetilde{X}_{k+1}$ is the blow-up of $\widetilde{X}_{k}$ along $W_{k}, D_{k+1}$ is the pre-image of $D_{k} \cup W_{k}$ by this blow-up, and $Y_{k+1}$ is the strict transform of $Y_{k}$;

(iv) $W_{k} \neq Y_{k}$ for $k=0, \ldots, N-1, W_{N-1}=Y_{N-1}$, and $W_{N}=\emptyset$.

These conditions imply that $Y_{N-1}$ is smooth and that $\widetilde{X}_{N}$ is an embedded resolution of singularities of $Y$. Moreover, the class of embedded resolutions of singularities of $Y$, which can be obtained by this method is cofinal among the class of all embedded resolutions of singularities of $Y$.

We apply Theorem 7.29 to the case when $X$ is a smooth real variety of dimension $d, D$ a fixed normal crossing divisor of $X$, and $Y$ an irreducible reduced subscheme of codimension $p$ in $X$ intersecting $D$ properly. Theorem 7.29 now provides an embedded resolution of singularities $\pi: \widetilde{X}_{N} \rightarrow X$ of $Y$ adapted to $D$. We note that $D_{N}=\pi^{-1}(Y \cup$ $D)$, and that $\pi^{-1}(Y)$ and $\pi^{-1}(D)$ have either no component or at least one component $E$ in common. In the latter case, the component $E$ appears in some intermediate step of the resolution of singularities, so we may assume that $E$ arises as the exceptional divisor from $W_{k}$ by the map $\pi_{k}: \widetilde{X}_{N} \rightarrow \widetilde{X}_{k}$ obtained by composing the corresponding blow-ups. Since $Y$ is not contained in $D$, we have $\operatorname{codim} W_{k}>\operatorname{codim} Y$. For later purposes, we denote by $\rho_{k}: \widetilde{X}_{k} \rightarrow X$ the map obtained by composing the corresponding remaining blow-ups, i.e. $\pi=\rho_{k} \circ \pi_{k}$.

We now describe the above situation in terms of local coordinates. We let $U$ denote a coordinate neighbourhood of $x \in \widetilde{X}_{N}$ with local coordinates $z_{1}, \ldots, z_{d}$ adapted to $D_{N}$. This means in particular that there are subsets $S$, respectively $T$, of $\{1, \ldots, d\}$ such that the normal crossing divisors $\pi^{-1}(Y)$, respectively $\pi^{-1}(D)$, are locally given by the equations

$$
\prod_{i \in S} z_{i}=0, \quad \text { respectively } \prod_{i \in T} z_{i}=0 .
$$


The case when $\pi^{-1}(Y)$ and $\pi^{-1}(D)$ have no component in common is then characterized by $S \cap T=\emptyset$, whereas in the other case we have $S \cap T \neq \emptyset$; in the latter case, we assume that $i \in S \cap T$, i.e. the common component $E$ is given by the equation $z_{i}=0$. Furthermore, we denote by $V$ a coordinate neighbourhood of $\pi_{k}(x) \in \widetilde{X}_{k}$ with local coordinates $t_{1}, \ldots, t_{d}$ adapted to the pair $\left(D_{k}, W_{k}\right)$; this means that $D_{k} \cap V$ is a union of coordinate hyperplanes and that $W_{k}$ is contained in the intersection of at least $p+1$ coordinate hyperplanes. For simplicity, we will assume that $W_{k}$ is contained in the subset defined by $t_{1}=\cdots=t_{p+1}=0$. After shrinking $U$, if necessary, we may assume that $\pi_{k}(U) \subseteq V$; furthermore, we may assume that $\pi_{k}\left(z_{1}, \ldots, z_{d}\right)=\left(t_{1}, \ldots, t_{d}\right)$. The condition $\pi_{k}(E) \subseteq W_{k}$ implies that these local coordinates satisfy

$$
\left(t_{1}, \ldots, t_{d}\right)=\left(z_{i}^{n_{1}} u_{1}, \ldots, z_{i}^{n_{p+1}} u_{p+1}, *, \ldots, *\right),
$$

where $n_{1}, \ldots, n_{p+1}$ are positive integers and $u_{1}, \ldots, u_{p+1}$ are holomorphic functions, whose divisor of zeros does not contain $E$.

\section{Basic pre-log-log Green forms}

In many cases, we can derive a formula for the push-forward of a $*$-product of top degree, which is similar to the push-forward of the *-product defined by Gillet and Soulé. A basic ingredient to derive such formula is the concept of basic pre-log-log Green forms, which we define below. These forms are the analogues of the basic Green forms introduced in $[\mathbf{1 5}]$.

Definition 7.31. Let $X$ be a smooth real variety with fixed normal crossing divisor $D$ as above, $y$ a $p$-codimensional cycle of $X$, and $Y=\operatorname{supp} y$. A basic pre-log-log Green form for $y$ is a differential form $g_{y}$ on $X$ satisfying:

(i) $\left(-2 \partial \bar{\partial} g_{y}, \widetilde{g_{y}}\right)$ is a Green object with values in $\mathcal{D}_{\text {pre }}$ for the cycle $y$;

(ii) there exists an embedded resolution of singularities $\pi: \widetilde{X} \rightarrow X$ of $Y$ adapted to $D$ such that in any coordinate neighbourhood with coordinates $z_{1}, \ldots, z_{d}$ adapted to $\pi^{-1}(Y)$ the equality

$$
g_{y}=\sum_{i} \alpha_{i} \log \left(1 / r_{i}\right)+\beta
$$

holds, where $\alpha_{i}$ are smooth differential forms on $\widetilde{X}$ and $\beta$ is a pre-log-log form on $\widetilde{X}$.

We note that in equation (7.31), the quantity $g_{y}$ should be replaced by $\pi^{*}\left(g_{y}\right)$. We permit ourselves this slight abuse of notation here and in the subsequent considerations in order to make our notation less heavy. Note also that in view of Proposition 7.21, if condition (ii) is satisfied then condition (i) holds if any of the of the equations (7.21) or (7.22) is satisfied.

By the cofinality property in the stated above version of Hironaka's theorem, we may assume that the embedded resolution of singularities appearing in the preceding definition of a basic pre-log-log Green form is obtained by means of Theorem 7.29, i.e. we may 
assume $\widetilde{X}=\widetilde{X}_{N}$ and choose the local coordinates according to the discussion following Theorem 7.29. In particular, we can then rewrite equation (7.31) as follows:

$$
g_{y}=\sum_{i \in S} \alpha_{i} \log \left(1 / r_{i}\right)+\beta
$$

We note that $g_{y}$ is a basic Green form in the sense of [15], if $\beta$ is a smooth differential form. We observe that the function $-\log \|s\|$ is a basic pre-log-log Green form for $\operatorname{div}(s)$, if $\bar{L}=(L,\|\cdot\|)$ is a good hermitian line bundle and $s$ a non-vanishing, rational section of $L$.

Push-forward of a *-product

Theorem 7.33. Let $\underline{X}=(X, D)$ be a proper, smooth real variety of dimension $d$ with fixed normal crossing divisor $D$. Let $y$ be an irreducible $p$-codimensional cycle of $X$, $Y=\operatorname{supp} y$, and $g_{y}$ a basic pre-log-log Green form for $y$. Let $z$ be a $q$-codimensional cycle of $X, Z=\operatorname{supp} z$, and $\mathfrak{g}_{z}=\left(\omega_{z}, \tilde{g}_{z}\right)$ a Green object with values in $\mathcal{D}_{\text {pre }}$ for the cycle $z$. Assume that $p+q=d+1$, that $Y$ and $Z$ intersect properly, i.e. $Y \cap Z=\emptyset$, and that $Y$ intersects $D$ properly. Then, the following formula holds

$$
\frac{1}{(2 \pi \mathrm{i})^{d}} \int_{X} \mathfrak{g}_{y} * \mathfrak{g}_{z}=\frac{1}{(2 \pi \mathrm{i})^{q-1}} \int_{Y} g_{z}+\frac{1}{(2 \pi \mathrm{i})^{d}} \int_{X} g_{y} \wedge \omega_{z} .
$$

Proof. Let $\sigma_{Y Z}$ and $\sigma_{Z Y}$ be as in Lemma 6.11. Recalling $-2 \partial \bar{\partial}=(4 \pi \mathrm{i}) \mathrm{d}^{\mathrm{c}}$ in conjunction with Theorem 6.12 , which is also valid for pre-log-log forms, we obtain

$$
\mathfrak{g}_{y} * \mathfrak{g}_{z}=\left(\omega_{y} \wedge \omega_{z}, 4 \pi \mathrm{i}\left(\mathrm{d}^{\mathrm{c}}\left(\sigma_{Y Z} g_{y}\right) \wedge g_{z}+\sigma_{Z Y} g_{y} \wedge \mathrm{d}^{\mathrm{c}} g_{z}\right)^{\sim}\right)
$$

We have to investigate the integral

$$
\int_{X} \mathfrak{g}_{y} * \mathfrak{g}_{z}=4 \pi \mathrm{i} \int_{X}\left(\mathrm{~d}^{\mathrm{c}}\left(\sigma_{Y Z} g_{y}\right) \wedge g_{z}+\sigma_{Z Y} g_{y} \wedge \mathrm{d}^{\mathrm{c}} g_{z}\right) .
$$

In order to perform these calculations, we put

$$
X_{\varepsilon}=X \backslash\left(B_{\varepsilon}(D) \cup B_{\varepsilon}(Y) \cup B_{\varepsilon}(Z)\right),
$$

where $B_{\varepsilon}(\cdot)$ denotes an $\varepsilon$-neighbourhood of the quantity in question. We observe that on $X_{\varepsilon}$ we can split up the integral

$$
\int_{X_{\varepsilon}}\left(\mathrm{d}^{\mathrm{c}}\left(\sigma_{Y Z} g_{y}\right) \wedge g_{z}+\sigma_{Z Y} g_{y} \wedge \mathrm{d}^{\mathrm{c}} g_{z}\right)
$$

into two summands. For the first summand we obtain

$$
\begin{aligned}
\int_{X_{\varepsilon}} \mathrm{d}^{\mathrm{c}}\left(\sigma_{Y Z} g_{y}\right) \wedge g_{z} & =\int_{X_{\varepsilon}}\left(\mathrm{d}^{\mathrm{c}} g_{y} \wedge g_{z}-\mathrm{d}^{\mathrm{c}}\left(\sigma_{Z Y} g_{y}\right) \wedge g_{z}\right) \\
& =\int_{X_{\varepsilon}}\left(\mathrm{d}^{\mathrm{c}} g_{y} \wedge g_{z}-\mathrm{d}\left(\mathrm{d}^{\mathrm{c}}\left(\sigma_{Z Y} g_{y}\right) \wedge g_{z}\right)+\mathrm{d}^{\mathrm{c}}\left(\sigma_{Z Y} g_{y}\right) \wedge \mathrm{d} g_{z}\right) .
\end{aligned}
$$


For the second summand we find

$$
\begin{aligned}
\int_{X_{\varepsilon}} \sigma_{Z Y} g_{y} \wedge \mathrm{d}^{\mathrm{c}} g_{z} & =\int_{X_{\varepsilon}}\left(g_{y} \wedge \mathrm{d}^{\mathrm{c}} g_{z}-\sigma_{Y Z} g_{y} \wedge \mathrm{d}^{\mathrm{c}} g_{z}\right) \\
& =\int_{X_{\varepsilon}}\left(g_{y} \wedge \mathrm{d}^{\mathrm{c}} g_{z}-\mathrm{d}\left(\sigma_{Y Z} g_{y} \wedge \mathrm{d}^{\mathrm{c}} g_{z}\right)+\mathrm{d}\left(\sigma_{Y Z} g_{y}\right) \wedge \mathrm{d}^{\mathrm{c}} g_{z}\right) \\
& =\int_{X_{\varepsilon}}\left(g_{y} \wedge \mathrm{d}^{\mathrm{c}} g_{z}-\mathrm{d}\left(\sigma_{Y Z} g_{y} \wedge \mathrm{d}^{\mathrm{c}} g_{z}\right)+\mathrm{d}^{\mathrm{c}}\left(\sigma_{Y Z} g_{y}\right) \wedge \mathrm{d} g_{z}\right) .
\end{aligned}
$$

Summing up all the terms in question now yields

$$
\begin{aligned}
\int_{X_{\varepsilon}} & \left(\mathrm{d}^{\mathrm{c}}\left(\sigma_{Y Z} g_{y}\right) \wedge g_{z}+\sigma_{Z Y} g_{y} \wedge \mathrm{d}^{\mathrm{c}} g_{z}\right) \\
= & \int_{X_{\varepsilon}}\left(g_{y} \wedge \mathrm{d}^{\mathrm{c}} g_{z}+\mathrm{d}^{\mathrm{c}} g_{y} \wedge g_{z}+\mathrm{d}^{\mathrm{c}} g_{y} \wedge \mathrm{d} g_{z}\right)-\int_{X_{\varepsilon}} \mathrm{d}\left(\mathrm{d}^{\mathrm{c}}\left(\sigma_{Z Y} g_{y}\right) \wedge g_{z}+\sigma_{Y Z} g_{y} \wedge \mathrm{d}^{\mathrm{c}} g_{z}\right) \\
= & \int_{X_{\varepsilon}} g_{y} \wedge \mathrm{d}^{\mathrm{c}} g_{z}+\int_{X_{\varepsilon}} \mathrm{d}\left(\mathrm{d}^{\mathrm{c}}\left(\sigma_{Y Z} g_{y}\right) \wedge g_{z}-\sigma_{Y Z} g_{y} \wedge \mathrm{d}^{\mathrm{c}} g_{z}\right) .
\end{aligned}
$$

The claim of Theorem 7.33 will now follow as a consequence of the subsequent two lemmas.

Lemma 7.35. With the notation and assumptions of Theorem 7.33, the differential form $g_{y} \wedge \mathrm{d}^{\mathrm{c}} g_{z}$ is locally integrable on $X$.

Proof. In order to show that the differential form $g_{y} \wedge \mathrm{d}^{\mathrm{c}} g_{z}$ is locally integrable on $X$, it suffices to show that it is locally integrable on an embedded resolution of singularities $\pi: \widetilde{X}_{N} \rightarrow X$ of $Y \cup Z$ adapted to $D$ as described in Theorem 7.29. We let $U$ be a coordinate neighbourhood of $x \in \widetilde{X}_{N}$ with local coordinates $z_{1}, \ldots, z_{d}$ adapted to $D_{N}$ as described in the discussion following Theorem 7.29. Then, we have

$$
g_{y}=\sum_{i \in S} \alpha_{i} \log \left(1 / r_{i}\right)+\beta,
$$

where $\alpha_{i}$ and $\beta$ are as in Definition 7.31. Since the differential form $\beta \wedge \mathrm{d}^{\mathrm{c}} g_{z}$ has $\log -\log$ growth along $\pi^{-1}(D)$, it is locally integrable on $\widetilde{X}_{N}$.

In order to study the local integrability of the forms $\alpha_{i} \log \left(1 / r_{i}\right) \wedge \mathrm{d}^{\mathrm{c}} g_{z}(i \in S)$, we have to distinguish the following two cases.

Case 1: $i \notin T$. This is the easy case. Namely, if we write

$$
\alpha_{i} \log \left(1 / r_{i}\right) \wedge \mathrm{d}^{\mathrm{c}} g_{z}=f\left(z_{1}, \ldots, z_{d}\right) \mathrm{d} z_{1} \wedge \cdots \wedge \mathrm{d} \bar{z}_{d},
$$

then $f$ satisfies the estimate

$$
\left|f\left(z_{1}, \ldots, z_{d}\right)\right| \prec \log \left(1 / r_{i}\right) \prod_{j \in T} \frac{\log \left(\log \left(1 / r_{j}\right)\right)^{M}}{r_{j}^{2} \log \left(1 / r_{j}\right)^{2}}
$$

for some positive integer $M$. Therefore, $\alpha_{i} \log \left(1 / r_{i}\right) \wedge \mathrm{d}^{\mathrm{c}} g_{z}$ is locally integrable on $\widetilde{X}_{N}$. 
Case 2: $i \in T$. Let $E$ be the divisor given by the equation $z_{i}=0$; then $E$ is a common component of $\pi^{-1}(Y)$ and $\pi^{-1}(D)$. Letting $\widetilde{X}_{k}$ with the local coordinates $t_{1}, \ldots, t_{d}$ be as in the discussion following Theorem 7.29, the component $E$ is mapped to the subset $W_{k}$, which is contained in the subset defined by $t_{1}=\cdots=t_{p+1}=0$. In these coordinates, we now write

$$
\rho_{k}^{*}\left(\mathrm{~d}^{\mathrm{c}} g_{z}\right)=\sum_{K^{\prime}, L^{\prime}} k_{K^{\prime}, L^{\prime}}\left(t_{1}, \ldots, t_{d}\right) \mathrm{d} t_{K^{\prime}} \wedge \mathrm{d} \bar{t}_{L^{\prime}},
$$

where $K^{\prime}, L^{\prime} \subseteq\{1, \ldots, d\}$ are strictly ordered subsets of cardinality $q$. We fix $K^{\prime}, L^{\prime}$, and put $\eta=k_{K^{\prime}, L^{\prime}}\left(t_{1}, \ldots, t_{d}\right) \mathrm{d} t_{K^{\prime}} \wedge \mathrm{d} \bar{t}_{L^{\prime}}$. Since $q=d-p+1$, the subset $K^{\prime}$ contains at least two elements

$$
a, b \in\{1, \ldots, p+1\},
$$

which implies that $\eta$ contains the differentials $\mathrm{d} t_{a}$ and $\mathrm{d} t_{b}$.

We are left to show that $\alpha_{i} \log \left(1 / r_{i}\right) \wedge \pi_{k}^{*}(\eta)$ is locally integrable. To this end, we write

$$
\begin{aligned}
\alpha_{i} \log \left(1 / r_{i}\right) & =\sum_{I, J} g_{I, J}\left(z_{1}, \ldots, z_{d}\right) \mathrm{d} z_{I} \wedge \mathrm{d} \bar{z}_{J}, \\
\pi_{k}^{*}(\eta) & =\sum_{K, L} h_{K, L}\left(z_{1}, \ldots, z_{d}\right) \mathrm{d} z_{K} \wedge \mathrm{d} \bar{z}_{L},
\end{aligned}
$$

where $I, J \subseteq\{1, \ldots, d\}$ are strictly ordered subsets of cardinality $p-1$, and $K, L \subseteq$ $\{1, \ldots, d\}$ are strictly ordered subsets of cardinality $q$. In this way, we obtain the $(d, d)$ form

$$
\alpha_{i} \log \left(1 / r_{i}\right) \wedge \pi_{k}^{*}(\eta)=\sum_{\substack{I, J, K, L \\ I \cap K=\emptyset \\ J \cap L=\emptyset}} f_{I, J, K, L}\left(z_{1}, \ldots, z_{d}\right) \mathrm{d} z_{1} \wedge \cdots \wedge \mathrm{d} \bar{z}_{d}
$$

with $f_{I, J, K, L}=g_{I, J} \cdot h_{K, L}$. We now show that each function $f_{I, J, K, L}$ is locally integrable on $\widetilde{X}_{N}$. If $i \in I \cap J$, the local integrability of $f_{I, J, K, L}$ follows as in Case 1 . If $i \in I \cap L$ or $i \in J \cap K$, the local integrability of $f_{I, J, K, L}$ follows from the estimate

$$
\left|f_{I, J, K, L}\left(z_{1}, \ldots, z_{d}\right)\right| \prec \frac{\log \left(\log \left(1 / r_{i}\right)\right)^{M}}{r_{i}} \prod_{\substack{j \in T \\ j \neq i}} \frac{\log \left(\log \left(1 / r_{j}\right)\right)^{M}}{r_{j}^{2} \log \left(1 / r_{j}\right)^{2}} .
$$

We are left to investigate the last sub-case $i \in K \cap L$. Letting $a, b$ be as in (7.35), and recalling the relation $t_{a}=z_{i}^{n_{a}} u_{a}$ from (7.29), we first observe

$$
\begin{gathered}
\pi_{k}^{*}\left(\frac{\log \left(\log \left(1 /\left|t_{a}\right|\right)\right)^{M}}{t_{a} \log \left(1 /\left|t_{a}\right|\right)} \mathrm{d} t_{a}\right)=\frac{\left(\log \left(n_{a} \log \left(1 / r_{i}\right)+\log \left(1 /\left|u_{a}\right|\right)\right)\right)^{M}}{u_{a}\left(n_{a} \log \left(1 / r_{i}\right)+\log \left(1 /\left|u_{a}\right|\right)\right)} \sum_{j=1}^{d} \frac{\partial u_{a}}{\partial z_{j}} \mathrm{~d} z_{j} \\
+\frac{n_{a}\left(\log \left(n_{a} \log \left(1 / r_{i}\right)+\log \left(1 /\left|u_{a}\right|\right)\right)\right)^{M}}{z_{i}\left(n_{a} \log \left(1 / r_{i}\right)+\log \left(1 /\left|u_{a}\right|\right)\right)} \mathrm{d} z_{i}
\end{gathered}
$$

and a similar formula for the index $b$. If $j \neq i$ in the above sum, we obtain the estimate

$$
\left|\frac{\left(\log \left(n_{a} \log \left(1 / r_{i}\right)+\log \left(1 /\left|u_{a}\right|\right)\right)\right)^{M}}{u_{a}\left(n_{a} \log \left(1 / r_{i}\right)+\log \left(1 /\left|u_{a}\right|\right)\right)} \frac{\partial u_{a}}{\partial z_{j}}\right| \prec \frac{\prod_{k \in T} \log \left(\log \left(1 / r_{k}\right)\right)^{M^{\prime}}}{r_{j}\left(\log \left(1 / r_{i}\right)+\log \left(1 / r_{j}\right)\right)}
$$


with some positive integer $M^{\prime}$. Since the divisor of zeros of $u_{a}$ does not contain the component $E$, we obtain for $j=i$ the estimate

$$
\left|\frac{\left(\log \left(n_{a} \log \left(1 / r_{i}\right)+\log \left(1 /\left|u_{a}\right|\right)\right)\right)^{M}}{u_{a}\left(n_{a} \log \left(1 / r_{i}\right)+\log \left(1 /\left|u_{a}\right|\right)\right)} \frac{\partial u_{a}}{\partial z_{i}}\right| \prec \frac{\prod_{k \in T} \log \left(\log \left(1 / r_{k}\right)\right)^{M^{\prime}}}{\log \left(1 / r_{i}\right)+\log \left(1 / r_{j}\right)} .
$$

Moreover, for the last summand, we note the estimate

$$
\left|\frac{n_{a}\left(\log \left(n_{a} \log \left(1 / r_{i}\right)+\log \left(1 /\left|u_{a}\right|\right)\right)\right)^{M}}{z_{i}\left(n_{a} \log \left(1 / r_{i}\right)+\log \left(1 /\left|u_{a}\right|\right)\right)}\right| \prec \frac{\prod_{k \in T} \log \left(\log \left(1 / r_{k}\right)\right)^{M^{\prime}}}{r_{i} \log \left(1 / r_{i}\right)} .
$$

Now, we note that the differential form $f_{I, J, K, L}\left(z_{1}, \ldots, z_{d}\right) \mathrm{d} z_{1} \wedge \cdots \wedge \mathrm{d} \bar{z}_{d}$ is built up in particular by the differential forms $\pi_{k}^{*}\left(\cdots \mathrm{d} t_{a}\right)$ and $\pi_{k}^{*}\left(\cdots \mathrm{d} t_{b}\right)$ (cf. (7.36)). We then observe that the summand of the differential $\pi_{k}^{*}\left(\cdots \mathrm{d} t_{a}\right)$ containing $\mathrm{d} z_{i}$ is multiplied with the corresponding summands of $\pi_{k}^{*}\left(\cdots \mathrm{d} t_{b}\right)$ containing the differentials $\mathrm{d} z_{j}$ for $j \neq i$. Hence, the function $f_{I, J, K, L}$ can be decomposed as a sum of functions $F_{j}(j \neq i)$, each of which satisfy the estimate

$$
\begin{aligned}
&\left|F_{j}\left(z_{1}, \ldots, z_{d}\right)\right| \prec \frac{\log \left(1 / r_{i}\right)}{r_{i}^{2} \log \left(1 / r_{i}\right)^{2}} \frac{\left(\log \left(\log \left(1 / r_{i}\right)\right) \log \left(\log \left(1 / r_{j}\right)\right)\right)^{M^{\prime}}}{r_{j}\left(\log \left(1 / r_{i}\right)+\log \left(1 / r_{j}\right)\right)} \\
& \times \frac{1}{r_{j} \log \left(1 / r_{j}\right)} \prod_{\substack{k \in T \\
k \neq i, j}} \frac{\log \left(\log \left(1 / r_{k}\right)\right)^{M^{\prime}}}{r_{k}^{2} \log \left(1 / r_{k}\right)^{2}} .
\end{aligned}
$$

Finally, using the inequality between the arithmetic and the geometric mean, we obtain

$$
\left|F_{j}\left(z_{1}, \ldots, z_{d}\right)\right| \prec \frac{1}{r_{i}^{2} r_{j}^{2}\left(\log \left(1 / r_{i}\right) \log \left(1 / r_{j}\right)\right)^{1+\varepsilon}} \prod_{\substack{k \in T \\ k \neq i, j}} \frac{\log \left(\log \left(1 / r_{k}\right)\right)^{M^{\prime}}}{r_{k}^{2} \log \left(1 / r_{k}\right)^{2}}
$$

for some $\varepsilon>0$. Adding up, this proves the local integrability of $f_{I, J, K, L}$, and thus concludes the proof of the local integrability of the differential form $\alpha_{i} \log \left(1 / r_{i}\right) \wedge \mathrm{d}^{\mathrm{c}} g_{z}$ on $\widetilde{X}_{N}$.

Lemma 7.38. With the notation and assumptions of Theorem 7.33, the equality

$$
\lim _{\varepsilon \rightarrow 0} \int_{X_{\varepsilon}} \mathrm{d}\left(\mathrm{d}^{\mathrm{c}}\left(\sigma_{Y Z} g_{y}\right) \wedge g_{z}-\sigma_{Y Z} g_{y} \wedge \mathrm{d}^{\mathrm{c}} g_{z}\right)=\frac{(2 \pi \mathrm{i})^{p-1}}{2} \int_{Y} g_{z}
$$

holds true.

Proof. For any $\varepsilon, \rho>0$, we put

$$
\begin{aligned}
X_{\varepsilon, \rho} & =X \backslash\left(B_{\varepsilon}(D) \cup B_{\rho}(Y) \cup B_{\varepsilon}(Z)\right), \\
Y_{\varepsilon} & =Y \backslash\left(B_{\varepsilon}(D) \cup B_{\varepsilon}(Z)\right) .
\end{aligned}
$$


Since $\sigma_{Y Z}$ vanishes in a neighbourhood of $Z$, we obtain by means of Stokes's theorem

$$
\begin{aligned}
& \int_{X_{\varepsilon, \rho}} \mathrm{d}\left(\mathrm{d}^{\mathrm{c}}\left(\sigma_{Y Z} g_{y}\right) \wedge g_{z}-\sigma_{Y Z} g_{y} \wedge \mathrm{d}^{\mathrm{c}} g_{z}\right) \\
&=\int_{\partial\left(B_{\varepsilon}(D) \cup B_{\rho}(Y)\right)}\left(\mathrm{d}^{\mathrm{c}}\left(\sigma_{Y Z} g_{y}\right) \wedge g_{z}-\sigma_{Y Z} g_{y} \wedge \mathrm{d}^{\mathrm{c}} g_{z}\right) .
\end{aligned}
$$

Since we derive from equation (7.33) in conjunction with Lemma 7.35 that the limit

$$
\lim _{\varepsilon, \rho \rightarrow 0} \int_{\partial\left(B_{\varepsilon}(D) \cup B_{\rho}(Y)\right)}\left(\mathrm{d}^{\mathrm{c}}\left(\sigma_{Y Z} g_{y}\right) \wedge g_{z}-\sigma_{Y Z} g_{y} \wedge \mathrm{d}^{\mathrm{c}} g_{z}\right)
$$

exists, we can compute it as an iterated limit by taking first the limit as $\rho$ tends to zero, and then the limit as $\varepsilon$ goes to zero. Since $g_{y}$ is a basic Green form in the sense of [15] for the cycle $y$ on $X \backslash\left(B_{\varepsilon}(D) \cup B_{\varepsilon}(Z)\right)$, Proposition 5.58 (ii) shows

$$
\begin{gathered}
\lim _{\rho \rightarrow 0} \int_{\partial\left(B_{\varepsilon}(D) \cup B_{\rho}(Y)\right)}\left(\mathrm{d}^{\mathrm{c}}\left(\sigma_{Y Z} g_{y}\right) \wedge g_{z}-\sigma_{Y Z} g_{y} \wedge \mathrm{d}^{\mathrm{c}} g_{z}\right) \\
=\int_{\partial B_{\varepsilon}(D)}\left(\mathrm{d}^{\mathrm{c}}\left(\sigma_{Y Z} g_{y}\right) \wedge g_{z}-\sigma_{Y Z} g_{y} \wedge \mathrm{d}^{\mathrm{c}} g_{z}\right)+\frac{(2 \pi \mathrm{i})^{p-1}}{2} \int_{Y_{\varepsilon}} g_{z} .
\end{gathered}
$$

By the functoriality and local integrability of the pre-log-log forms, we have

$$
\lim _{\varepsilon \rightarrow 0} \int_{Y_{\varepsilon}} g_{z}=\int_{Y} g_{z}
$$

Therefore, we are left to show that

$$
\lim _{\varepsilon \rightarrow 0} \int_{\partial B_{\varepsilon}(D)}\left(\mathrm{d}^{\mathrm{c}}\left(\sigma_{Y Z} g_{y}\right) \wedge g_{z}-\sigma_{Y Z} g_{y} \wedge \mathrm{d}^{\mathrm{c}} g_{z}\right)=0 .
$$

To perform this calculation, we will again work on an embedded resolution of singularities $\pi: \widetilde{X}_{N} \rightarrow X$ of $Y \cup Z$ adapted to $D$ together with a coordinate neighbourhood $U$ of $x \in \widetilde{X}_{N}$ with local coordinates $z_{1}, \ldots, z_{d}$ adapted to $D_{N}$. Following the discussion after Theorem 7.29, we may assume without loss of generality that the situation is local, i.e.

$$
\pi^{-1}(D)=\bigcup_{i \in T} E_{i}
$$

where $E_{i}$ is given by the equation $z_{i}=0(i \in T)$; the choice of local coordinates determines differentiable maps

$$
p_{i}: \partial B_{\varepsilon}\left(E_{i}\right) \rightarrow E_{i} \quad(i \in T)
$$

From this we obtain (recalling our abuse of notation)

$$
\begin{aligned}
\lim _{\varepsilon \rightarrow 0} \int_{\partial B_{\varepsilon}(D)}\left(\mathrm{d}^{\mathrm{c}}\left(\sigma_{Y Z} g_{y}\right) \wedge\right. & \left.g_{z}-\sigma_{Y Z} g_{y} \wedge \mathrm{d}^{\mathrm{c}} g_{z}\right) \\
= & \sum_{i \in T} \lim _{\varepsilon \rightarrow 0} \int_{\partial B_{\varepsilon}\left(E_{i}\right)}\left(\mathrm{d}^{\mathrm{c}}\left(\sigma_{Y Z} g_{y}\right) \wedge g_{z}-\sigma_{Y Z} g_{y} \wedge \mathrm{d}^{\mathrm{c}} g_{z}\right) \\
= & \sum_{i \in T} \lim _{\varepsilon \rightarrow 0} \int_{E_{i}} \int_{p_{i}}\left(\mathrm{~d}^{\mathrm{c}}\left(\sigma_{Y Z} g_{y}\right) \wedge g_{z}-\sigma_{Y Z} g_{y} \wedge \mathrm{d}^{\mathrm{c}} g_{z}\right) .
\end{aligned}
$$


We will now fix $i_{0} \in T$, and show that

$$
\int_{p_{i_{0}}}\left|\mathrm{~d}^{\mathrm{c}}\left(\sigma_{Y Z} g_{y}\right) \wedge g_{z}-\sigma_{Y Z} g_{y} \wedge \mathrm{d}^{\mathrm{c}} g_{z}\right| \prec h_{1}(\varepsilon) h_{2},
$$

where $\lim _{\varepsilon \rightarrow 0} h_{1}(\varepsilon)=0$ and $h_{2}$ is locally integrable on $E_{i_{0}}$; we emphasize that equality (7.38) is an inequality between forms meaning that the inequality holds true for the absolute values of all the components involved. To do this, we observe that the differential form

$$
\mathrm{d}^{\mathrm{c}}\left(\sigma_{Y Z} g_{y}\right) \wedge g_{z}-\sigma_{Y Z} g_{y} \wedge \mathrm{d}^{\mathrm{c}} g_{z}
$$

is the sum of two forms, one of which is of type $(d, d-1)$, and the other of type $(d-1, d)$. We will prove the lemma for the term of type $(d, d-1)$ noting that the argument for the term of type $(d-1, d)$ is analogous.

Since $g_{y}$ is a basic pre-log-log Green form, we have (as in the proof of Lemma 7.35)

$$
g_{y}=\sum_{i \in S} \alpha_{i} \log \left(1 / r_{i}\right)+\beta
$$

Therefore, we have to establish the bound (7.38) for each summand of $g_{y}$ in the above decomposition. For the summand $\beta$ the bound (7.38) follows easily, since $\beta$ is a pre-log-log form. In order to treat the remaining summands, we write

$$
\begin{gathered}
\sigma_{Y Z} g_{y} \wedge \partial g_{z}=f\left(z_{1}, \ldots, z_{d}\right) \mathrm{d} z_{i_{0}} \wedge \prod_{\substack{j=1 \\
j \neq i_{0}}}^{d} \mathrm{~d} z_{j} \wedge \mathrm{d} \bar{z}_{j}+\cdots, \\
\partial\left(\sigma_{Y Z} g_{y}\right) \wedge g_{z}=g\left(z_{1}, \ldots, z_{d}\right) \mathrm{d} z_{i_{0}} \wedge \prod_{\substack{j=1 \\
j \neq i_{0}}}^{d} \mathrm{~d} z_{j} \wedge \mathrm{d} \bar{z}_{j}+\cdots .
\end{gathered}
$$

As in the previous lemma, we have to distinguish two cases.

Case 1: $i \notin T$. Then, we easily find the estimates

$$
\begin{aligned}
\left|f\left(z_{1}, \ldots, z_{d}\right)\right| \prec \log \left(1 / r_{i}\right) \frac{\log \left(\log \left(1 / r_{i_{0}}\right)\right)^{M}}{r_{i_{0}} \log \left(1 / r_{i_{0}}\right)} \prod_{\substack{j \in T \\
j \neq i_{0}}} \frac{\log \left(\log \left(1 / r_{j}\right)\right)^{M}}{r_{j}^{2} \log \left(1 / r_{j}\right)^{2}}, \\
\left|g\left(z_{1}, \ldots, z_{d}\right)\right| \prec \frac{1}{r_{i}} \frac{\log \left(\log \left(1 / r_{i_{0}}\right)\right)^{M}}{r_{i_{0}} \log \left(1 / r_{i_{0}}\right)} \prod_{\substack{j \in T \\
j \neq i_{0}}} \frac{\log \left(\log \left(1 / r_{j}\right)\right)^{M}}{r_{j}^{2} \log \left(1 / r_{j}\right)^{2}},
\end{aligned}
$$

from which the bound (7.38) follows.

Case 2: $i \in T$. In this case, the functions $f$ and $g$ will satisfy the same type of estimates; thus, we will discuss here only the estimate for the function $f$. If $i=i_{0}$, by arguing as in the second part of the discussion of Case 2 in the proof of Lemma 7.35, we find that $f$ can 
be decomposed as a sum of functions $F_{j}(j \neq i)$, each of which satisfying the estimate

$$
\begin{aligned}
&\left|F_{j}\left(z_{1}, \ldots, z_{d}\right)\right| \prec \frac{\log \left(1 / r_{i_{0}}\right)}{r_{i_{0}} \log \left(1 / r_{i_{0}}\right)} \frac{\left(\log \left(\log \left(1 / r_{i_{0}}\right)\right) \log \left(\log \left(1 / r_{j}\right)\right)\right)^{M^{\prime}}}{r_{j}\left(\log \left(1 / r_{i_{0}}\right)+\log \left(1 / r_{j}\right)\right)} \quad \times \frac{1}{r_{j} \log \left(1 / r_{j}\right)} \prod_{\substack{k \in T \\
k \neq i_{0}, j}} \frac{\log \left(\log \left(1 / r_{k}\right)\right)^{M^{\prime}}}{r_{k}^{2} \log \left(1 / r_{k}\right)^{2}} \\
& \prec \frac{1}{r_{i_{0}} r_{j}^{2} \log \left(1 / r_{i_{0}}\right)^{\varepsilon} \log \left(1 / r_{j}\right)^{1+\varepsilon}} \prod_{\substack{k \in T \\
k \neq i_{0}, j}} \frac{\log \left(\log \left(1 / r_{k}\right)\right)^{M^{\prime}}}{r_{k}^{2} \log \left(1 / r_{k}\right)^{2}} ;
\end{aligned}
$$

here $M^{\prime}$ and $\varepsilon$ are as in the proof of Lemma 7.35. If $i \neq i_{0}$, the function $f$ can be decomposed as a sum of functions $F_{j}(j \neq i)$, which are seen to satisfy for $j=i_{0}$ the estimate

$$
\left|F_{i_{0}}\left(z_{1}, \ldots, z_{d}\right)\right| \prec \frac{1}{r_{i_{0}} r_{i}^{2} \log \left(1 / r_{i_{0}}\right)^{\varepsilon} \log \left(1 / r_{i}\right)^{1+\varepsilon}} \prod_{\substack{k \in T \\ k \neq i_{0}, i}} \frac{\log \left(\log \left(1 / r_{k}\right)\right)^{M^{\prime}}}{r_{k}^{2} \log \left(1 / r_{k}\right)^{2}},
$$

and for $j \neq i_{0}$ the estimate

$$
\left|F_{j}\left(z_{1}, \ldots, z_{d}\right)\right| \prec \frac{1}{r_{i_{0}} r_{i}^{2} r_{j}^{2} \log \left(1 / r_{i_{0}}\right) \log \left(1 / r_{i}\right)^{1+\varepsilon} \log \left(1 / r_{j}\right)^{1+\varepsilon}} \prod_{\substack{k \in T \\ k \neq i_{0}, i, j}} \frac{\log \left(\log \left(1 / r_{k}\right)\right)^{M^{\prime}}}{r_{k}^{2} \log \left(1 / r_{k}\right)^{2}}
$$

All in all, this leads to the desired estimate (7.38) and concludes the proof of the lemma.

Remark 7.40. Clearly, the theorem also holds when $D=\emptyset$, and in particular for Green objects with values in $\mathcal{D}_{\log }$. In this case we recover the classical formula for the $*$-product of Green currents used in [36] and [71] (see also [16,17]). If $X$ is a Riemann surface, then formula (7.33) implies the formulae for the generalized arithmetic intersection numbers given in $[58]$.

\subsection{Arithmetic Chow rings with pre-log-log forms}

\section{Definition of arithmetic Chow groups with pre-log-log forms}

Let $\left(A, \Sigma, F_{\infty}\right)$ be an arithmetic ring and $X$ an arithmetic variety over $A$. Let $D$ be a fixed normal crossing divisor of $X_{\Sigma}$, which is stable under $F_{\infty}$. Following the notation of the previous section we will denote the pair $\left(X_{\mathbb{R}}, D\right)$ by $\underline{X}$. The natural inclusion $\mathcal{D}_{\text {log }} \rightarrow \mathcal{D}_{\text {pre }}$ induces a $\mathcal{D}_{\text {log }}$-complex structure in $\mathcal{D}_{\text {pre }}$. Then, $\left(X, \mathcal{D}_{\text {pre }}\right)$ is a

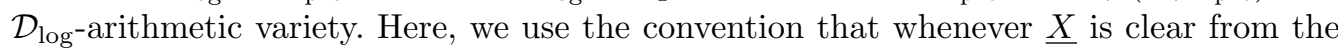
context, we write $\mathcal{D}_{\text {pre }}$ instead of $\mathcal{D}_{\text {pre, }, \underline{X}}$ Therefore, applying the theory developed in $\S 4$, we can define the arithmetic Chow groups $\widehat{\mathrm{CH}}^{*}\left(X, \mathcal{D}_{\text {pre }}\right)$.

The rest of this section will be devoted to studying the basic properties of the arithmetic Chow groups $\widehat{\mathrm{CH}}^{*}\left(X, \mathcal{D}_{\text {pre }}\right)$. In particular, we will see that they satisfy properties similar to ones of the classical arithmetic Chow groups stated in [36] and most of the properties stated in [61]. 
Exact sequences

We will use the notation of $\S 6.1$ applied to the sheaf $\mathcal{D}_{\text {pre }}$. We start by writing the exact sequences given in Theorem 4.13.

Theorem 7.41. Let $X$ be an arithmetic variety over $A$, and $\underline{X}=\left(X_{\mathbb{R}}, D\right)$ as above. Then, we have the following exact sequences:

$$
\begin{aligned}
& \mathrm{CH}^{p-1, p}(X) \stackrel{\rho}{\rightarrow} \widetilde{\mathcal{D}}_{\text {pre }}^{2 p-1}(X, p)^{\text {pure }} \stackrel{\text { a }}{\rightarrow} \widehat{\mathrm{CH}}^{p}\left(X, \mathcal{D}_{\text {pre }}\right) \stackrel{\zeta}{\rightarrow} \mathrm{CH}^{p}(X) \rightarrow 0, \\
& \mathrm{CH}^{p-1, p}(X) \stackrel{\rho}{\rightarrow} H_{\mathcal{D}_{\text {pre }}}^{2 p-1}(X, p)^{\text {pure }} \stackrel{\text { a }}{\rightarrow} \widehat{\mathrm{CH}}^{p}\left(X, \mathcal{D}_{\text {pre }}\right) \\
& \stackrel{(\zeta,-\omega)}{\rightarrow} \mathrm{CH}^{p}(X) \oplus \mathrm{Z}_{\text {pre }}^{2 p}(X, p) \stackrel{\text { cl }+h}{\rightarrow} H_{\mathcal{D}_{\text {pre }}}^{2 p}(X, p) \rightarrow 0, \\
& \mathrm{CH}^{p-1, p}(X) \stackrel{\rho}{\rightarrow} H_{\mathcal{D}_{\text {pre }}}^{2 p-1}(X, p)^{\text {pure }} \stackrel{\text { a }}{\rightarrow} \widehat{\mathrm{CH}}^{p}\left(X, \mathcal{D}_{\text {pre }}\right)_{0} \stackrel{\zeta}{\rightarrow} \mathrm{CH}^{p}(X)_{0} \rightarrow 0 .
\end{aligned}
$$

Proof. This is a direct consequence of Theorem 4.13.

Since $\mathcal{D}_{\text {pre }}$ is a $\mathcal{D}_{\text {log }}$-complex, there is a morphism

$$
H_{\mathcal{D}}^{*}\left(X_{\mathbb{R}}, p\right) \rightarrow H_{\mathcal{D}_{\text {pre }}}^{*}(X, p) .
$$

Thus, from Theorem 7.41 we obtain the following corollary.

Corollary 7.42. There is a complex of abelian groups

$$
H_{\mathcal{D}}^{2 p-1}\left(X_{\mathbb{R}}, p\right) \stackrel{\text { a }}{\rightarrow} \widehat{\mathrm{CH}}^{p}\left(X, \mathcal{D}_{\text {pre }}\right) \stackrel{(\zeta,-\omega)}{\longrightarrow} \mathrm{CH}^{p}(X) \oplus \mathrm{ZD}_{\text {pre }}^{2 p}(X, p) .
$$

Remark 7.43. Since we have not computed the cohomology of the complex $\mathcal{D}_{\text {pre }}$, these exact sequences do not give all the possible information. We do not even know whether the corresponding cohomology groups satisfy the weak purity condition.

As we have already pointed out, by imposing bounds on all derivatives of the functions, one can define a smaller complex, the complex of log-log forms, for which we can prove the Poincaré Lemma. Using this complex one obtains arithmetic Chow groups that satisfy the exact sequences of Theorem 7.41, but with real Deligne-Beilinson cohomology (see Remark 7.20). This will be developed in a forthcoming paper [19].

Green forms for a cycle

We now translate the result of Proposition 7.21 into the language of Green objects.

Proposition 7.44. Let $X$ be an arithmetic variety over $A$, and $y$ a $p$-codimensional cycle on $X$. If a pair $\mathfrak{g}=(\omega, \widetilde{g}) \in \widehat{H}_{\mathcal{D}_{\text {pre }}, \mathcal{Z}^{p}}^{2 p}(X, p)$ is a Green object for the class of $y$, then

$$
-2 \partial \bar{\partial}[g]_{X}=[\omega]-\delta_{y} .
$$

We recall that in the above proposition $[g]_{X}$ stands for $[g]_{X_{\infty}}$, and $\delta_{y}$ for $\delta_{y_{\mathbb{R}}}$. 
Multiplicative properties

By Theorem 7.19 , the complex $\mathcal{D}_{\text {pre }}$ satisfies all the properties required to apply Theorem 4.24 .

Theorem 7.46. The abelian group

$$
\widehat{\mathrm{CH}}^{*}\left(X, \mathcal{D}_{\text {pre }}\right)_{\mathbb{Q}}=\bigoplus_{p \geqslant 0} \widehat{\mathrm{CH}}^{p}\left(X, \mathcal{D}_{\text {pre }}\right) \otimes \mathbb{Q}
$$

is a commutative and associative $\mathbb{Q}$-algebra with unit.

Inverse images

Let $f: X \rightarrow Y$ be a morphism of arithmetic varieties over $A$. By Proposition 7.12, the complex $\mathcal{D}_{\text {pre }}$ satisfies all the properties required to apply Theorem 4.29.

Theorem 7.47. Let $f: X \rightarrow Y$ be a morphism of arithmetic varieties over $A$, let $D_{X}$, $D_{Y}$ be a normal crossing divisor on $X_{\mathbb{R}}, Y_{\mathbb{R}}$, respectively, satisfying $f^{-1}\left(D_{Y}\right) \subseteq D_{X}$. Put $\underline{X}=\left(X_{\mathbb{R}}, D_{X}\right)$ and $\underline{Y}=\left(Y_{\mathbb{R}}, D_{Y}\right)$. Then, there is an inverse image morphism

$$
f^{*}: \widehat{\mathrm{CH}}^{*}\left(Y, \mathcal{D}_{\text {pre }, \underline{Y}}\right) \rightarrow \widehat{\mathrm{CH}}^{*}\left(X, \mathcal{D}_{\text {pre }, \underline{X}}\right) .
$$

Moreover, this morphism is a morphism of rings after tensoring with $\mathbb{Q}$.

Push-forward

We will state only the consequence of Proposition 7.27.

Theorem 7.48. Let $X$ be a $d$-dimensional projective arithmetic variety over $A$, $\underline{X}=\left(X_{\mathbb{R}}, D\right)$ as above, and $\pi: X \rightarrow \operatorname{Spec}(A)$ the structural morphism. Then, there is a direct image morphism

$$
\pi_{*}: \widehat{\mathrm{CH}}^{d+1}\left(X, \mathcal{D}_{\text {pre }}\right) \rightarrow \widehat{\mathrm{CH}}^{1}(\operatorname{Spec}(A)) .
$$

Proof. Since $\operatorname{Spec}(A)_{\mathbb{R}}$ is a finite collection of points, the complex $\mathcal{D}_{\log , \operatorname{Spec}(A)}$ is constant. By Proposition 7.27, there is a covariant morphism of arithmetic varieties

$$
\pi:\left(X, \mathcal{D}_{\text {pre }}\right) \rightarrow\left(\operatorname{Spec}(A), \mathcal{D}_{\log }\right) .
$$

The claim now follows from Theorem 4.34.

Relationship with other arithmetic Chow groups

Let $X$ be an arithmetic variety over $A$, and $\underline{X}=\left(X_{\mathbb{R}}, D\right)$ as above. The structural morphism

$$
\mathcal{D}_{\log } \rightarrow \mathcal{D}_{\text {pre }}
$$

induces a morphism of arithmetic Chow groups

$$
\widehat{\mathrm{CH}}^{*}\left(X, \mathcal{D}_{\log }\right) \rightarrow \widehat{\mathrm{CH}}^{*}\left(X, \mathcal{D}_{\text {pre }}\right),
$$


which is compatible with inverse images, intersection products and arithmetic degrees. If $X$ is projective, the isomorphism between $\widehat{\mathrm{CH}}^{*}\left(X, \mathcal{D}_{\log }\right)$ and the arithmetic Chow groups defined by Gillet and Soulé (denoted by $\widehat{\mathrm{CH}}^{*}(X)$ ) induces a morphism

$$
\widehat{\mathrm{CH}}^{*}(X) \rightarrow \widehat{\mathrm{CH}}^{*}\left(X, \mathcal{D}_{\text {pre }}\right)
$$

which is also compatible with inverse images, intersection products and arithmetic degrees. Since we have not proven the purity of the cohomology of $\mathcal{D}_{\text {pre }}$, we cannot state that the above morphism is an isomorphism, when $D$ is trivial, except when the dimension of $X_{F}$ is zero (see Remarks 7.20 and 7.43).

\section{Arithmetic Picard group}

The theory of line bundles equipped with smooth hermitian metrics developed in $\S 6.1$ can be generalized to the case of good metrics. For this let $X$ be a projective arithmetic variety over $A, \underline{X}=\left(X_{\mathbb{R}}, D\right)$ as above, and $L$ a line bundle on $X$ equipped with a hermitian metric $h$ on the induced line bundle $L_{\infty}$ over $X_{\infty}$, which is good along $D$ and invariant under $F_{\infty}$. As usual, we write $\bar{L}=(L,\|\cdot\|)$, and refer to it as a good hermitian line bundle. Given a rational section $s$ of $L$, we write $\|s\|^{2}=h(s, s)$ for the point-wise norm of the induced section of $L_{\infty}$. We say that two good hermitian line bundles $\bar{L}_{1}$ and $\bar{L}_{2}$ are isometric, if $\bar{L}_{1} \otimes \bar{L}_{2}^{-1} \cong\left(\mathcal{O}_{X},|\cdot|\right)$, where $|\cdot|$ is the standard absolute value. The arithmetic Picard group $\widehat{\operatorname{Pic}}(\underline{X})$ is the group of isometry classes of good hermitian line bundles with group structure given by the tensor product.

Proposition 7.50. Let $X$ be a projective arithmetic variety over $A, \underline{X}=\left(X_{\mathbb{R}}, D\right)$ as above, and $\bar{L}=(L,\|\cdot\|)$ a good hermitian line bundle on $X$. Then, there is a map

$$
\widehat{\mathrm{c}}_{1}: \widehat{\operatorname{Pic}}(\underline{X}) \rightarrow \widehat{\mathrm{CH}}^{1}\left(X, \mathcal{D}_{\text {pre }}\right)
$$

given by sending the class of $\bar{L}$ to the class $\left[\operatorname{div}(s),\left(\omega_{s}, \widetilde{g}_{s}\right)\right] \in \widehat{\mathrm{CH}}^{1}\left(X, \mathcal{D}_{\text {pre }}\right)$, where $s$, $\omega_{s}$, and $g_{s}$ are as in Proposition 7.25.

Proof. We have to show that the map $\widehat{c}_{1}$ is well defined. By Proposition 7.25, the pair $\left(\omega_{s}, g_{s}\right)$ represents a Green object for the class of $\operatorname{div}(s)$. If $s^{\prime}$ is another non-vanishing, rational section, then $s^{\prime}=f \cdot s$ with a rational function $f$, and we have

$$
\left(\operatorname{div}\left(s^{\prime}\right),\left(\omega_{s^{\prime}}, \widetilde{g}_{s^{\prime}}\right)\right)=\left(\operatorname{div}(s),\left(\omega_{s}, \widetilde{g}_{s}\right)\right)+\widehat{\operatorname{div}}(f) .
$$

Hence, $\left(\operatorname{div}(s),\left(\omega_{s}, \widetilde{g}_{s}\right)\right)$ and $\left(\operatorname{div}\left(s^{\prime}\right),\left(\omega_{s^{\prime}}, \widetilde{g}_{s^{\prime}}\right)\right)$ represent the same class in the arithmetic Chow group $\widehat{\mathrm{CH}}^{1}\left(X, \mathcal{D}_{\text {pre }}\right)$. Therefore, the map $\widehat{\mathrm{c}}_{1}$ is well defined.

Definition 7.51. Let $X$ be a projective arithmetic variety over $A, \underline{X}=\left(X_{\mathbb{R}}, D\right)$ as above, and $\bar{L}$ a good hermitian line bundle on $X$. Then $\widehat{c}_{1}(\bar{L}) \in \widehat{\mathrm{CH}}^{1}\left(X, \mathcal{D}_{\text {pre }}\right)$ is called the first arithmetic Chern class of $\bar{L}$. 


\section{Arithmetic degree}

Let $K$ be a number field, $\mathcal{O}_{K}$ its ring of integers, and $S=\operatorname{Spec}\left(\mathcal{O}_{K}\right)$. From $\S 6.1$, we recall the arithmetic degree map

$$
\widehat{\operatorname{deg}}: \widehat{\mathrm{CH}}^{1}\left(S, \mathcal{D}_{\log }\right) \rightarrow \mathbb{R} \text {. }
$$

Definition 7.52. Let $X$ be a $d$-dimensional projective arithmetic variety over $\mathcal{O}_{K}$ with structural morphism $\pi: X \rightarrow S, \underline{X}=\left(X_{\mathbb{R}}, D\right)$ as above, and $\bar{L}$ a good hermitian line bundle on $X$. The real number

$$
\bar{L}^{d+1}=\widehat{\operatorname{deg}}\left(\pi_{*} \widehat{\mathrm{c}}_{1}(\bar{L})^{d+1}\right)
$$

is called the arithmetic degree of $\bar{L}$, or the arithmetic self-intersection number of $\bar{L}$.

Remark 7.53. Logarithmically singular line bundles on arithmetic surfaces as defined in $[\mathbf{5 8}]$ are good hermitian line bundles. It is straightforward to show that the intersection number at the infinite places given in [58] (see [58, Lemma 3.9]), agrees with the explicit formula for the $*$-product given in Theorem 7.33 . We also note that by $[\mathbf{5 8}$, Proposition 7.4] the arithmetic self-intersection number of a good hermitian line bundle calculated by either of the formulae in $[\mathbf{9}],[\mathbf{6 3}],[\mathbf{5 8}]$, or Theorem 7.33 agree.

\section{Height pairings}

Let $K$ be a number field, $\mathcal{O}_{K}$ its ring of integers, and $S=\operatorname{Spec}\left(\mathcal{O}_{K}\right)$. Let $X$ be a $d$ dimensional projective arithmetic variety over $\mathcal{O}_{K}$ with structural morphism $\pi: X \rightarrow S$. Furthermore, fix a normal crossing divisor $D$ of $X_{\mathbb{R}}$.

We want to generalize the height pairing given in Definition 6.39 to the arithmetic Chow groups with values in $\mathcal{D}_{\text {pre }}$. This generalization will in particular include the logarithmic heights for points considered by Faltings in $[\mathbf{2 8}, \mathbf{3 0}]$. Since the height of a cycle, whose generic part is supported in $D$, might be infinite, one cannot expect that the height pairing (6.39) unconditionally generalizes to a height pairing between the arithmetic Chow groups $\widehat{\mathrm{CH}}^{p}\left(X, \mathcal{D}_{\text {pre }}\right)$ and the whole group of cycles $\mathrm{Z}^{q}(X)$. Therefore, we restrict ourselves to considering a subgroup of cycles, for which a height pairing with respect to $\widehat{\mathrm{CH}}^{p}\left(X, \mathcal{D}_{\text {pre }}\right)$ can always be defined.

Putting $U=X_{\mathbb{R}} \backslash D$, we write $\mathrm{Z}_{U}^{q}(X)$ for the group of the $q$-codimensional cycles $z$ of $X$ such that $z_{\mathbb{R}}$ intersects $D$ properly. A case of particular interest is given when the normal crossing divisor $D$ is defined over $K$; we then introduce $U_{K}=X_{K} \backslash D_{K}$, and observe that there is a natural injective map

$$
\mathrm{Z}^{q}\left(U_{K}\right) \rightarrow \mathrm{Z}_{U}^{q}(X)
$$

Definition 7.54. We want to define a bilinear pairing

$$
(\cdot \mid \cdot): \widehat{\mathrm{CH}}^{p}\left(X, \mathcal{D}_{\text {pre }}\right) \otimes \mathrm{Z}_{U}^{q}(X) \rightarrow \widehat{\mathrm{CH}}^{p+q-d}\left(S, \mathcal{D}_{\log }\right)_{\mathbb{Q}} .
$$

Let $z \in \mathrm{Z}_{U}^{q}(X)$ be an irreducible, reduced cycle and $\alpha \in \widehat{\mathrm{CH}}^{p}\left(X, \mathcal{D}_{\text {pre }}\right)$. We represent $\alpha$ by the class of an arithmetic cycle $\left(y, \mathfrak{g}_{y}\right)$, where $y$ is a $p$-codimensional cycle such that 
$y_{K}$ intersects $z_{K}$ properly and where $\mathfrak{g}_{y}=\left(\omega_{y}, \widetilde{g_{y}}\right)$ is a pre-log-log Green object for $y$. If $p+q<d$, we put $(\alpha \mid z)=0$. If $p+q=d$, we define

$$
(\alpha \mid z)=\left[\pi_{*}\left(y_{K} \cdot z_{K}\right),(0,0)\right] \in \widehat{\mathrm{CH}}^{0}\left(S, \mathcal{D}_{\log }\right)_{\mathbb{Q}}=\mathrm{CH}^{0}(S)_{\mathbb{Q}} .
$$

Finally, if $p+q=d+1$, we define

$$
\left.(\alpha \mid z)=\left[\pi_{*}\left([y \cdot z]_{\text {fin }}\right),\left(0, \pi_{\#} \widehat{\left(g_{y} \wedge\right.} \delta_{z}\right)\right)\right] \in \widehat{\mathrm{CH}}^{1}\left(S, \mathcal{D}_{\log }\right)_{\mathbb{Q}} .
$$

Here, the quantity $\left.\pi_{\#} \widetilde{\left(g_{y} \wedge\right.} \delta_{z}\right)$ has to be understood as follows: Let $Z=\operatorname{supp} z_{\mathbb{R}}$ and $\imath: \widetilde{Z} \rightarrow Z$ be a resolution of singularities of $Z$ adapted to $D$. Since $y_{K} \cap z_{K}=\emptyset$, the functoriality of pre-log-log forms shows that $\imath^{*}\left(g_{y}\right)$ is a pre-log-log form on $\widetilde{Z}$, hence it is locally integrable on $\widetilde{Z}$, and we have

$$
\left.\pi_{\#} \widetilde{\left(g_{y} \wedge\right.} \delta_{z}\right)=\frac{1}{(2 \pi \mathrm{i})^{p-1}} \int_{\widetilde{Z}} \imath^{*}\left(g_{y}\right) .
$$

The pairing (7.54) is now obtained by linearly extending the above definitions.

Note that this definition a priori depends on the choice of the representative $\left(y, \mathfrak{g}_{y}\right)$ of $\alpha$.

Proposition 7.56. With the above notation and assumptions, let $\alpha \in \widehat{\mathrm{CH}}^{p}\left(X, \mathcal{D}_{\text {pre }}\right)$, $z \in \mathrm{Z}_{U}^{q}(X), Z=\operatorname{supp} z_{\mathbb{R}}$, and $p+q=d+1$. If we choose a basic pre-log-log Green form $g_{z}$ for $z$ and put $\mathfrak{g}_{z}=\left(-2 \partial \bar{\partial} g_{z}, \widetilde{g}_{z}\right)$, the height pairing (7.54) satisfies the equality

$$
(\alpha \mid z)=\pi_{*}\left(\alpha \cdot\left[z, \mathfrak{g}_{z}\right]\right)+\mathrm{a}\left(\pi_{\#}\left(\left[\omega\left(\widetilde{\alpha) \wedge g_{z}}\right]_{X}\right)\right)\right.
$$

in $\widehat{\mathrm{CH}}^{1}\left(S, \mathcal{D}_{\log }\right)_{\mathbb{Q}}$.

Proof. By Lemma 7.35, the pre-log form $\omega(\alpha) \wedge g_{z}$ is integrable. Therefore, the righthand side of the claimed formula is well defined. We now represent $\alpha$ by the arithmetic cycle $\left(y, \mathfrak{g}_{y}\right)$, where $y$ is a $p$-codimensional cycle such that $y_{K}$ intersects $z_{K}$ properly and where $\mathfrak{g}_{y}=\left(\omega(\alpha), \widetilde{g_{y}}\right)$ is a pre-log-log Green object for the cycle $y$. Then, the first summand of the right-hand side of equation (7.56) equals

$$
\pi_{*}\left(\alpha \cdot\left[z, \mathfrak{g}_{z}\right]\right)=\left[\pi_{*}\left([y \cdot z]_{\mathrm{fin}}\right),\left(0, \frac{1}{(2 \pi \mathrm{i})^{d}} \int_{X} \mathfrak{g}_{y} * \mathfrak{g}_{z}\right)\right] .
$$

Using Theorem 7.33 (with the roles of $y$ and $z$ interchanged), i.e. the equality

$$
\frac{1}{(2 \pi \mathrm{i})^{d}} \int_{X} \mathfrak{g}_{y} * \mathfrak{g}_{z}=\frac{1}{(2 \pi \mathrm{i})^{p-1}} \int_{Z} g_{y}+\frac{1}{(2 \pi \mathrm{i})^{d}} \int_{X} \omega(\alpha) \wedge g_{z},
$$

we find

$$
\begin{aligned}
\pi_{*}\left(\alpha \cdot\left[z, \mathfrak{g}_{z}\right]\right. & )+\mathrm{a}\left(\pi_{\#}\left(\left[\omega\left(\widetilde{\alpha) \wedge g_{z}}\right]_{X}\right)\right)\right. \\
= & {\left[\pi_{*}\left([y \cdot z]_{\text {fin }}\right),\left(0, \widetilde{\pi_{\#}} \widetilde{\left(\widetilde{g_{y} \wedge}\right.} \delta_{z}\right)+\pi_{\#}\left(\left[\omega\left(\widetilde{\alpha) \wedge g_{z}}\right]_{X}\right)\right)\right]+\mathrm{a}\left(\pi_{\#}\left(\left[\omega\left(\widetilde{\alpha) \wedge g_{z}}\right]_{X}\right)\right)\right.} \\
= & {\left.\left[\pi_{*}\left([y \cdot z]_{\text {fin }}\right),\left(0, \pi_{\#} \widetilde{\left(g_{y} \wedge\right.} \delta_{z}\right)\right)\right] } \\
= & (\alpha \mid z),
\end{aligned}
$$

which proves the claim. 
In the following theorem, we will show that the pairing (7.54) is well defined.

Theorem 7.58. The bilinear pairing (7.54) is well defined. Furthermore, there is a commutative diagram

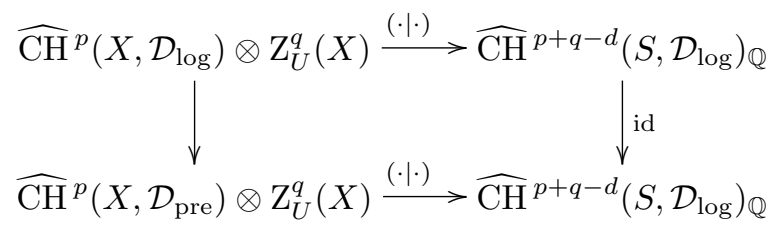

where horizontal maps are given by the pairings (6.39) and (7.54), respectively.

Proof. We have to show that the height pairing $(\alpha \mid z)$ does not depend on the choice of a representative $\left(y, \mathfrak{g}_{y}\right)$ for $\alpha$. Since the height pairing $(7.54)$ coincides with the height pairing (6.39) for $p, q$ satisfying $p+q \leqslant d$, it suffices to treat the case $p+q=d+1$. In order to prove the well-definedness in the latter case, we recall that the height pairing satisfies formula (7.56). By the well-definedness of the arithmetic intersection product and the map $\omega$, the right-hand side of (7.56) turns out to be independent of the representative $\left(y, \mathfrak{g}_{y}\right)$, which proves the well-definedness of $(\alpha \mid z)$.

The commutativity of the diagram follows from Lemma 6.42 observing the formula

$$
\mathrm{a}\left(\left[g_{z}\right]_{X}(\omega(\alpha))\right)=\mathrm{a}\left(\pi_{\#}\left(\left[\omega\left(\widetilde{\alpha) \wedge} g_{z}\right]_{X}\right)\right) .\right.
$$

\section{Faltings heights}

We keep the notation of the previous section. In particular, we let $X, D, U$ be as before and $p, q$ integers satisfying $p+q=d+1$. The height pairing given by Definition 7.54 is of particular interest, when $\alpha=\widehat{\mathrm{c}}_{1}(\bar{L})^{p}$ for some good hermitian line bundle $\bar{L}$ on $X$. If $z \in \mathrm{Z}_{U}^{q}(X)$, we call the real number

$$
\mathrm{ht}_{\bar{L}}(z)=\widehat{\operatorname{deg}}\left(\widehat{\mathrm{c}}_{1}(\bar{L})^{p} \mid z\right)
$$

the Faltings height of $z$ (with respect to $\bar{L}$ ). This name is justified by the following result due to Faltings.

Proposition 7.59. In addition to the data fixed above, assume that $L$ is an ample line bundle. Then, for any $c \in \mathbb{R}$, the cardinality of the set

$$
\left\{z \in \mathrm{Z}_{U}^{d}(X) \mid \mathrm{ht}_{\bar{L}}(z)<c\right\}
$$

is finite.

Proof. Since the metric of a good hermitian line bundle is logarithmically singular as defined by Faltings, the proof is standard (see, for example, [69]). 


\subsection{Application to products of modular curves}

As an example, we show in this final section how the arithmetic intersection theory with pre-log-log forms developed in this section can be applied to compute the arithmetic self-intersection number of the line bundle of modular forms equipped with the natural invariant metric on the product of two modular curves. We point out that because of the singularities of the Petersson metric, these numbers are only well defined in the setting of our newly developed extended arithmetic intersection theory. Related but more elaborate results for the case of Hilbert modular surfaces have recently been obtained in [13]. Further calculations of other naturally metrized automorphic line bundles have been carried out in [12]. The general theory of arithmetic characteristic classes of automorphic vector bundles of arbitrary rank is developed in [19].

\section{Modular curves}

Let $\mathbb{H}$ denote the upper half-plane with complex coordinate $z=x+\mathrm{i} y$, and

$$
X(1)=\mathrm{SL}_{2}(\mathbb{Z}) \backslash \mathbb{H} \cup\left\{S_{\infty}\right\}
$$

the modular curve with the cusp $S_{\infty}$. For more details on the subsequent facts we refer to $[58]$.

Let $\mathcal{X}(1)=\mathbb{P}_{\mathbb{Z}}^{1}$ be the regular model for the modular curve $X(1)$. With $s_{\infty}$ denoting the Zariski closure of (the normal crossing divisor) $S_{\infty} \subseteq X(1)$ and $k$ a positive integer satisfying $12 \mid k$, we define the line bundle of modular forms of weight $k$ by $\mathcal{M}_{k}=$ $\mathcal{O}\left(s_{\infty}\right)^{\otimes k / 12}$. The line bundle $\mathcal{M}_{k}$ is equipped with the Petersson metric $\|\cdot\|$, which is a good hermitian metric along $S_{\infty}$; hence, $\overline{\mathcal{M}}_{k}=\left(\mathcal{M}_{k},\|\cdot\|\right)$ is a good hermitian line bundle, and we have

$$
\widehat{\mathrm{c}}_{1}\left(\overline{\mathcal{M}}_{k}\right) \in \widehat{\mathrm{CH}}^{1}\left(\mathcal{X}(1), \mathcal{D}_{\text {pre }}\right) .
$$

For the first Chern form $c_{1}\left(\overline{\mathcal{M}}_{k}\right)$ of $\overline{\mathcal{M}}_{k}$, we recall the formulae

$$
\mathrm{c}_{1}\left(\overline{\mathcal{M}}_{k}\right)=k \frac{\mathrm{d} z \wedge \mathrm{d} \bar{z}}{(z-\bar{z})^{2}}=-\frac{k}{2 \mathrm{i}} \frac{\mathrm{d} x \wedge \mathrm{d} y}{y^{2}}, \quad \int_{X(1)} \mathrm{c}_{1}\left(\overline{\mathcal{M}}_{k}\right)=-\frac{\pi k}{6 \mathrm{i}} .
$$

Fixing a $(1,1)$-form $\omega$ satisfying $\int_{X(1)} \omega=-2 \pi \mathrm{i}$, the main result of [58] shows

$$
\widehat{\mathrm{c}}_{1}\left(\overline{\mathcal{M}}_{k}\right)^{2}=\left[0, \overline{\mathcal{M}}_{k}^{2} \cdot \mathrm{a}(\omega)\right] \in \widehat{\mathrm{CH}}^{2}\left(\mathcal{X}(1), \mathcal{D}_{\text {pre }}\right)_{\mathbb{Q}}
$$

where the arithmetic self-intersection number is given by

$$
\overline{\mathcal{M}}_{k}^{2}=k^{2}\left(\frac{1}{2} \zeta_{\mathbb{Q}}(-1)+\zeta_{\mathbb{Q}}^{\prime}(-1)\right)
$$

with the Riemann zeta function $\zeta_{\mathbb{Q}}(s)$. 
Products of modular curves

In this example we consider the arithmetic threefold $\mathcal{H}=\mathcal{X}(1) \times_{\mathbb{Z}} \mathcal{X}(1)$; we let $p_{1}$, respectively $p_{2}$, denote the projection onto the first, respectively second, factor. The divisor

$$
D=p_{1}^{*} \mathcal{X}(1) \times p_{2}^{*} s_{\infty}+p_{1}^{*} s_{\infty} \times p_{2}^{*} \mathcal{X}(1)
$$

induces a normal crossing divisor $D_{\mathbb{R}}$ on $\mathcal{H}_{\mathbb{R}}$. For $k, l \in \mathbb{N}, 12|k, 12| l$, we define the hermitian line bundle

$$
\overline{\mathcal{L}}(k, l)=p_{1}^{*} \overline{\mathcal{M}}_{k} \otimes p_{2}^{*} \overline{\mathcal{M}}_{l} .
$$

It can be easily checked that the hermitian metric of $\overline{\mathcal{L}}(k, l)$ is good along $D_{\mathbb{R}}$.

Theorem 7.61. Let $\mathcal{H}$ and $\overline{\mathcal{L}}(k, l)$ be as above. Then, the arithmetic self-intersection number of $\overline{\mathcal{L}}(k, l)$ is well defined, and given by the formula

$$
\overline{\mathcal{L}}(k, l)^{3}=\frac{1}{4}\left(k^{2} l+l^{2} k\right)\left(\frac{1}{2} \zeta_{\mathbb{Q}}(-1)+\zeta_{\mathbb{Q}}^{\prime}(-1)\right) .
$$

Proof. By Theorem 7.47, we have

$$
\widehat{\mathrm{c}}_{1}(\overline{\mathcal{L}}(k, l)) \in \widehat{\mathrm{CH}}^{1}\left(\mathcal{H}, \mathcal{D}_{\text {pre }}\right) ;
$$

this can also be checked directly. Letting $\pi: \mathcal{H} \rightarrow \operatorname{Spec}(\mathbb{Z})$ denote the structural morphism, the arithmetic self-intersection number of $\overline{\mathcal{L}}(k, l)$ is given by

$$
\overline{\mathcal{L}}(k, l)^{3}=\widehat{\operatorname{deg}}\left(\pi_{*} \widehat{c}_{1}(\overline{\mathcal{L}}(k, l))^{3}\right),
$$

which is well defined by Theorem 7.48. Using the properties of arithmetic Chow groups, we derive the following identities in $\widehat{\mathrm{CH}}^{3}\left(\mathcal{H}, \mathcal{D}_{\text {pre }}\right)_{\mathbb{Q}}$ :

$$
\begin{aligned}
\widehat{\mathrm{c}}_{1}(\overline{\mathcal{L}}(k, l))^{3} & =\left(p_{1}^{*} \widehat{\mathrm{c}}_{1}\left(\overline{\mathcal{M}}_{k}\right)+p_{2}^{*} \widehat{\mathrm{c}}_{1}\left(\overline{\mathcal{M}}_{l}\right)\right)^{3} \\
& =\sum_{j=0}^{3}\left(\begin{array}{l}
3 \\
j
\end{array}\right) p_{1}^{*} \widehat{\mathrm{c}}_{1}\left(\overline{\mathcal{M}}_{k}\right)^{j} \cdot p_{2}^{*} \widehat{\mathrm{c}}_{1}\left(\overline{\mathcal{M}}_{l}\right)^{3-j} \\
& =\sum_{j=1}^{2}\left(\begin{array}{l}
3 \\
j
\end{array}\right) p_{1}^{*} \widehat{\mathrm{c}}_{1}\left(\overline{\mathcal{M}}_{k}\right)^{j} \cdot p_{2}^{*} \widehat{\mathrm{c}}_{1}\left(\overline{\mathcal{M}}_{l}\right)^{3-j} .
\end{aligned}
$$

By means of formula (7.59), we find

$$
\widehat{\mathrm{c}}_{1}(\overline{\mathcal{L}}(k, l))^{3}=\left(\begin{array}{l}
3 \\
2
\end{array}\right)\left(\left[0, \overline{\mathcal{M}}_{k}^{2} \cdot \mathrm{a}\left(p_{1}^{*} \omega\right)\right] \cdot p_{2}^{*} \widehat{\mathrm{c}}_{1}\left(\overline{\mathcal{M}}_{l}\right)+p_{1}^{*} \widehat{\mathrm{c}}_{1}\left(\overline{\mathcal{M}}_{k}\right) \cdot\left[0, \overline{\mathcal{M}}_{l}^{2} \cdot \mathrm{a}\left(p_{2}^{*} \omega\right)\right]\right) .
$$

Applying Proposition 6.10, we obtain

$$
\widehat{\mathrm{c}}_{1}(\overline{\mathcal{L}}(k, l))^{3}=3\left(\left[0, \overline{\mathcal{M}}_{k}^{2} \cdot \mathrm{a}\left(p_{1}^{*} \omega \wedge p_{2}^{*} \mathrm{c}_{1}\left(\overline{\mathcal{M}}_{l}\right)\right)\right]+\left[0, \overline{\mathcal{M}}_{l}^{2} \cdot \mathrm{a}\left(p_{1}^{*} \mathrm{c}_{1}\left(\overline{\mathcal{M}}_{k}\right) \wedge p_{2}^{*} \omega\right)\right]\right)
$$


Taking the push-forward of the latter quantity by $\pi$ and applying the arithmetic degree map, we finally obtain

$$
\begin{aligned}
\overline{\mathcal{L}}(k, l)^{3}=\frac{3}{(2 \pi \mathrm{i})^{2}} & \left(\frac{1}{2} \zeta_{\mathbb{Q}}(-1)+\zeta_{\mathbb{Q}}^{\prime}(-1)\right) \\
& \times\left(k^{2} \int_{X(1)}(-\omega) \int_{X(1)} \mathrm{c}_{1}\left(\overline{\mathcal{M}}_{l}\right)+l^{2} \int_{X(1)} \mathrm{c}_{1}\left(\overline{\mathcal{M}}_{k}\right) \int_{X(1)}(-\omega)\right),
\end{aligned}
$$

from which the claimed formula follows.

Faltings heights of Hecke correspondences

Let $N$ be a positive integer, and $M_{N}$ the set of integral $(2 \times 2)$-matrices of determinant $N$. Recall that the group $\mathrm{SL}_{2}(\mathbb{Z})$ acts form the right on the set $M_{N}$ and that a complete set of representatives for this action is given by the set

$$
R_{N}=\left\{\gamma=\left(\begin{array}{ll}
a & b \\
0 & d
\end{array}\right) \mid a, b, d \in \mathbb{Z} ; a d=N ; d>0 ; 0 \leqslant b<d\right\}
$$

The cardinality $\sigma(N)$ of $R_{N}$ is given by

$$
\sigma(N)=\sum_{d \mid N} d
$$

where the sum is taken over all positive divisors $d$ of $N$. Furthermore, we put

$$
T_{N}=\left\{\left(z_{1}, z_{2}\right) \in \mathbb{H} \times \mathbb{H} \mid \exists \gamma \in M_{N}: z_{1}=\gamma z_{2}\right\}
$$

This defines a divisor on $X(1) \times X(1)$, whose normalization is a finite sum of modular curves $X_{0}(m)$ associated to the congruence subgroups $\Gamma_{0}(m)$ for $m$ dividing $N$. These curves are also referred to as the graphs of the Hecke correspondences. Denoting the discriminant by $\Delta(z)$ and the $j$-function by $j(z)$, the Hilbert modular form

$$
s_{N}\left(z_{1}, z_{2}\right)=\Delta\left(z_{1}\right)^{\sigma(N)} \Delta\left(z_{2}\right)^{\sigma(N)} \prod_{\gamma \in R_{N}}\left(j\left(\gamma z_{1}\right)-j\left(z_{2}\right)\right)
$$

has divisor $T_{N}$. Since the Fourier coefficients of $s_{N}\left(z_{1}, z_{2}\right)$ are integral, it defines a section of $\mathcal{L}(12 \sigma(N), 12 \sigma(N))$; we put

$$
\mathcal{T}_{N}=\operatorname{div}\left(s_{N}\right) \subseteq \mathcal{H}
$$

Theorem 7.62. The Faltings height of the Hecke correspondence $\mathcal{T}_{N}$ with respect to $\overline{\mathcal{L}}(k, k)$ is given by the formula

$$
\mathrm{ht}_{\overline{\mathcal{L}}(k, k)}\left(\mathcal{T}_{N}\right)=(2 k)^{2}\left(\sigma(N)\left(\frac{1}{2} \zeta_{\mathbb{Q}}(-1)+\zeta_{\mathbb{Q}}^{\prime}(-1)\right)+\sum_{d \mid N} \frac{d \log (d)}{24}-\frac{\sigma(N) \log (N)}{48}\right) .
$$


Proof. Using Rohrlich's modular Jensen's formula, we obtain for any fixed $z_{0} \in \mathbb{H}$

$$
\begin{aligned}
& \int_{X(1)} \log \left\|s_{N}\left(z, z_{0}\right)\right\| \frac{\mathrm{d} x}{4 \pi y^{2}} \\
&=-12 \sigma(N)\left(\frac{1}{2} \zeta_{\mathbb{Q}}(-1)+\zeta_{\mathbb{Q}}^{\prime}(-1)\right)+\frac{1}{12} \sum_{\gamma \in R_{N}} \log \left(\frac{\|\Delta(z)\|}{\|\Delta(\gamma z)\|}\right) .
\end{aligned}
$$

Since we have

$$
\prod_{\gamma \in R_{N}} \frac{\Delta(z)}{\Delta(\gamma z)}=1, \quad \operatorname{Im}\left(\frac{a z+b}{d}\right)=\frac{N}{d^{2}} \operatorname{Im} z \quad\left(\left(\begin{array}{ll}
a & b \\
0 & d
\end{array}\right) \in R_{N}\right),
$$

we obtain for the sum on the right-hand side

$$
\begin{aligned}
\frac{1}{12} \sum_{\gamma \in R_{N}} \log \left(\frac{\|\Delta(z)\|}{\|\Delta(\gamma z)\|}\right) & =\frac{1}{12} \log \left|\prod_{\gamma \in R_{N}} \frac{\Delta(z)}{\Delta(\gamma z)}\right|+\frac{1}{2} \sum_{\gamma \in R_{N}} \log \left(\frac{d^{2}}{N}\right) \\
& =\sum_{d \mid N} d \log (d)-\frac{1}{2} \sigma(N) \log (N) .
\end{aligned}
$$

Writing $z_{1}=x_{1}+\mathrm{i} y_{1}, z_{2}=x_{2}+\mathrm{i} y_{2}$, and observing

$$
\mathrm{c}_{1}(\overline{\mathcal{L}}(k, k))^{2}=-\frac{k^{2}}{2} \frac{\mathrm{d} x_{1} \wedge \mathrm{d} y_{1}}{y_{1}^{2}} \wedge \frac{\mathrm{d} x_{2} \wedge \mathrm{d} y_{2}}{y_{2}^{2}},
$$

we find by means of Rohrlich's formula

$$
\begin{aligned}
& \frac{1}{(2 \pi \mathrm{i})^{2}} \int_{X(1) \times X(1)} \log \left\|s_{N}\left(z_{1}, z_{2}\right)\right\| \cdot \mathrm{c}_{1}(\overline{\mathcal{L}}(k, k))^{2} \\
& \quad=k^{2}\left(-2 \sigma(N)\left(\frac{1}{2} \zeta_{\mathbb{Q}}(-1)+\zeta_{\mathbb{Q}}^{\prime}(-1)\right)+\frac{1}{6} \sum_{d \mid N} d \log (d)-\frac{1}{12} \sigma(N) \log (N)\right) .
\end{aligned}
$$

Since $s_{N}$ defines a section of $\mathcal{L}(12 \sigma(N), 12 \sigma(N))$ and $\log \left\|s_{N}\left(z_{1}, z_{2}\right)\right\|$ is a basic pre-log-log form, we obtain by Proposition 7.56 and the previous considerations

$$
\begin{aligned}
\mathrm{ht}_{\overline{\mathcal{L}}(k, k)}\left(\mathcal{T}_{N}\right)= & \widehat{\operatorname{deg}}\left(\widehat{\mathrm{c}}_{1}(\overline{\mathcal{L}}(k, k))^{2} \mid \mathcal{T}_{N}\right) \\
= & \widehat{\operatorname{deg}}\left(\pi_{*}\left(\widehat{\mathrm{c}}_{1}(\overline{\mathcal{L}}(k, k))^{2} \cdot\left[\mathcal{T}_{N},\left(\mathrm{c}_{1}(\overline{\mathcal{L}}(12 \sigma(N), 12 \sigma(N))),-\log \left\|s_{N}\left(z_{1}, z_{2}\right)\right\|\right)\right]\right)\right. \\
& \left.+\mathrm{a}\left(\pi_{\#}\left(\left[-\log \left\|s_{N}\left(z_{1}, z_{2}\right)\right\| \wedge \mathrm{c}_{1}(\overline{\mathcal{L}}(k, k))^{2}\right] X(1) \times X(1)\right)\right)\right) \\
= & \widehat{\operatorname{deg}}\left(\pi_{*}\left(\widehat{\mathrm{c}}_{1}(\overline{\mathcal{L}}(k, k))^{2} \cdot \widehat{\mathrm{c}}_{1}(\overline{\mathcal{L}}(12 \sigma(N), 12 \sigma(N)))\right)\right) \\
& +\frac{1}{(2 \pi \mathrm{i})^{2}} \int_{X(1) \times X(1)} \log \left\|s_{N}\left(z_{1}, z_{2}\right)\right\| \cdot \mathrm{c}_{1}(\overline{\mathcal{L}}(k, k))^{2} \\
= & 6 k^{2} \sigma(N)\left(\frac{1}{2} \zeta_{\mathbb{Q}}(-1)+\zeta_{\mathbb{Q}}^{\prime}(-1)\right) \\
& +k^{2}\left(-2 \sigma(N)\left(\frac{1}{2} \zeta_{\mathbb{Q}}(-1)+\zeta_{\mathbb{Q}}^{\prime}(-1)\right)+\frac{1}{6} \sum_{d \mid N} d \log (d)-\frac{1}{12} \sigma(N) \log (N)\right) .
\end{aligned}
$$


Remark 7.63. We point out that the same formula has been obtained independently by Autissier [3]. His calculations, which are based on results of Kühn [58] and use Bost's theory of $L_{1}^{2}$-singular metrics on arithmetic surfaces [9], are compatible with definition (7.54). We also note that the arithmetic intersection numbers obtained via Bost's $L_{1}^{2}$ theory applied to logarithmically singular metrics equal those in [58] and coincide with those obtained by applying the theory developed here in the two-dimensional setting.

Arithmetic generating series

We put

$$
\widehat{T(N)}= \begin{cases}0, & \text { if } N<0 \\ {\left[\mathcal{T}_{N}, \mathfrak{g}_{N}\right],} & \text { if } N>0\end{cases}
$$

where

$$
\left.\mathfrak{g}_{N}(z)=\left(2 \partial \bar{\partial} \log \left\|s_{N}\left(z_{1}, z_{2}\right)\right\|,-\log \widetilde{\| s_{N}\left(z_{1}\right.}, z_{2}\right) \|\right) .
$$

With the divisor $D \subseteq \mathcal{H}$, we additionally put

$$
\widehat{T(0)}=-\frac{1}{24} \widehat{\mathrm{c}}_{1}(\overline{\mathcal{L}}(12,12))+\frac{1}{8 \pi y}\left[D, \mathfrak{g}_{D}(z)\right] \in \widehat{\mathrm{CH}}^{1}\left(\mathcal{H}, \mathcal{D}_{\text {pre }}\right)_{\mathbb{R}}
$$

where

$$
\mathfrak{g}_{D}(z)=\left(2 \partial \bar{\partial} \log \left\|\Delta\left(z_{1}\right) \Delta\left(z_{2}\right)\right\|,-\log \left\|\widetilde{\Delta\left(z_{1}\right)} \Delta\left(z_{2}\right)\right\|\right) .
$$

With these ingredients we define the following generating series of arithmetic cycles

$$
\widehat{\phi}_{\text {can }}(q)=\sum_{N \in \mathbb{Z}} \widehat{T(N)} q^{N} \in \widehat{\mathrm{CH}}^{1}\left(\mathcal{H}, \mathcal{D}_{\text {pre }}\right)_{\mathbb{R}} \otimes \mathbb{C}((q)) ;
$$

here $q=\mathrm{e}^{2 \pi \mathrm{i} z}$.

Theorem 7.64. The generating series $\widehat{\phi}_{\text {can }}$ is a non-holomorphic modular form with values in $\widehat{\mathrm{CH}}^{1}\left(\mathcal{H}, \mathcal{D}_{\text {pre }}\right)_{\mathbb{R}}$ of weight 2 for $\mathrm{SL}_{2}(\mathbb{Z})$.

Proof. We observe that

$$
\widehat{T(N)}=\widehat{\mathrm{c}}_{1}(\overline{\mathcal{L}}(12,12)) \cdot \begin{cases}0, & \text { if } N<0 \\ \left(-\frac{1}{24}+\frac{1}{8 \pi y}\right), & \text { if } N=0 \\ \sigma(N), & \text { if } N>0 .\end{cases}
$$

Recall now that there is the non-holomorphic Eisenstein series $E_{2}(z)$ of weight 2 obtained by means of Hecke's convergence trick from the non-holomorphic Eisenstein series $E_{2}(z, s)$ at $s=1$; it has Fourier expansion

$$
E_{2}(z, 1)=-\frac{1}{24}+\frac{1}{8 \pi y}+\sum_{N>0} \sigma(N) q^{N}
$$


Therefore, we obtain

$$
\widehat{\phi}_{\text {can }}(q)=\widehat{\mathrm{c}}_{1}(\overline{\mathcal{L}}(12,12)) \otimes E_{2}(z) \in \widehat{\mathrm{CH}}^{1}\left(\mathcal{H}, \mathcal{D}_{\text {pre }}\right)_{\mathbb{R}} \otimes \mathcal{A}_{2}\left(\mathrm{SL}_{2}(\mathbb{Z})\right),
$$

where $\mathcal{A}_{2}\left(\mathrm{SL}_{2}(\mathbb{Z})\right)$ denotes the space of non-holomorphic modular forms of weight 2 with respect to $\mathrm{SL}_{2}(\mathbb{Z})$.

By Theorem 7.64 any linear functional on $\widehat{\mathrm{CH}}^{1}\left(\mathcal{H}, \mathcal{D}_{\text {pre }}\right)_{\mathbb{R}}$ applied to $\widehat{\phi}_{\text {can }}$ gives rise to a modular form of weight 2 . As possible applications we present an integral version of Borcherds's generating series

$$
\zeta\left(\widehat{\phi}_{\text {can }}\right)=-\frac{1}{24} c_{1}(\mathcal{L}(12,12))+\frac{1}{8 \pi y} D+\sum_{N>0} \mathcal{T}_{N} q^{N} \in \mathrm{CH}(\mathcal{H})_{\mathbb{R}} \otimes \mathcal{A}_{2}\left(\mathrm{SL}_{2}(\mathbb{Z})\right),
$$

or the Hirzebruch-Zagier formula, which states that for any $(1,1)$-form $\eta$, we have

$$
[\eta]_{\mathcal{H}_{\infty}}\left(\operatorname{cl}\left(\zeta\left(\widehat{\phi}_{\text {can }}\right)\right)\right)=\sum_{N \geqslant 0}\left(\frac{1}{2 \pi \mathrm{i}} \int_{T_{N}} \eta\right) q^{N} \in \mathcal{A}_{2}\left(\mathrm{SL}_{2}(\mathbb{Z})\right) .
$$

In particular, we emphasize that choosing $\eta=c_{1}(\overline{\mathcal{L}}(12,12))$ yields a multiple of the Eisenstein series $E_{2}(z, 1)$.

We conclude by noting that according to Kudla's conjectures there should also exist a generating series related to the derivative of $E_{2}(z, s)$ at $s=1$.

Acknowledgements. This work was partially supported by grants DGI BFM20000799-C02-01 and BFM2003-02914.

In the course of preparing this manuscript, we had many stimulating discussions with many colleagues. We would like to thank them all. In particular, we express our gratitude to J.-B. Bost, W. Gubler, M. Harris, S. Kudla, V. Maillot, D. Roessler and C. Soulé. We would also like to thank G. Freixas for his careful proof reading of the manuscript. Furthermore, we would like to thank to EAGER, Arithmetic Geometry Network, Newton Institute, and the Institut Henri Poincaré for partial support of our work.

\section{References}

1. S. J. Arakelov, An intersection theory for divisors on an arithmetic surface, Math. USSR Izv. 8 (1974), 1167-1180.

2. M. Artin, A. Grothendieck and J. L. Verdier, Théorie des topos et cohomologie étale des schémas, Lecture Notes in Mathematics, Volumes 269, 270, 305 (Springer, 19721973).

3. P. Autissier, Hauteur des correspondances de Hecke, Bull. Soc. Math. France 131(3) (2003), 421-433.

4. A. A. Beilinson, Higher regulators and values of $L$-functions, J. Sov. Math. 30 (1985), 2036-2070.

5. P. Berthelot, A. Grothendieck And L. Illusie, Théorie des intersections et théorème de Riemann-Roch, Lecture Notes in Mathematics, Volume 225 (Springer, 1971).

6. S. Bloch And A. Ogus, Gersten's conjecture and the homology of schemes, Annls Scient. Éc. Norm. Sup. 7 (1974), 181-202. 
7. S. Bloch, H. Gillet And C. Soulé, Non archimedean Arakelov theory, J. Alg. Geom. 5 (1995), 427-485.

8. T. Bloom And M. Herrera, De Rham cohomology of an analytic space, Invent. Math. 7 (1969), 275-296.

9. J.-B. Bost, Potential theory and Lefschetz theorems for arithmetic surfaces, Annls Scient. Éc. Norm. Sup. 32 (1999), 241-312.

10. J.-B. Bost, H. Gillet And C. Soulé, Heights of projective varieties and positive Green forms, J. Am. Math. Soc. 7 (1994), 903-1027.

11. K. S. Brown And S. M. Gersten, Algebraic $K$-theory as generalized sheaf cohomology, in Algebraic K-theory I (ed. H. Bass), Lecture Notes in Mathematics, Volume 341, pp. 266-292 (Springer, 1973).

12. J. BRUinier AND U. KüHn, Integrals of automorphic Green's functions associated to Heegner divisors, Int. Math. Res. Not. 31 (2003), 1687-1729.

13. J. Bruinier, J. I. Burgos GIL ANd U. KÜHN, Borcherds products and arithmetic intersection theory on Hilbert modular surfaces, preprint math.NT/0310201 (2003).

14. J. I. Burgos Gil, Arithmetic Chow rings, PhD thesis, University of Barcelona (1994).

15. J. I. Burgos Gil, A $C^{\infty}$-logarithmic Dolbeault complex, Compositio Math. 92 (1994), $61-86$.

16. J. I. Burgos GiL, Green forms and their product, Duke Math. J. 75 (1994), 529-574.

17. J. I. Burgos Gil, Arithmetic Chow rings and Deligne-Beilinson cohomology, J. Alg. Geom. 6 (1997), 335-377.

18. J. I. Burgos Gil ANd S. WANG, Higher Bott-Chern forms and Beilinson's regulator, Invent. Math. 132 (1998), 261-305.

19. J. I. Burgos Gil, J. KRAmer AND U. KüHN, Arithmetic characteristic classes of automorphic vector bundles, Documenta Math. 10 (2005), 619-716.

20. N. DAN, Prolongement méromorphe des courants de Green, Math. Ann. 323 (2002), 175199.

21. R. DE JEU, Zagier's conjecture and wedge complexes in algebraic $K$-theory, Compositio Math. 96 (1995), 197-247.

22. J. A. DE Jong, Smoothness, semi-stability and alterations, Publ. Math. IHES 83 (1996), 51-93.

23. P. Deligne, Théorie de Hodge, II, Publ. Math. IHES 40 (1972), 5-57.

24. P. Deligne, Théorie de Hodge, III, Publ. Math. IHES 44 (1975), 5-77.

25. P. Deligne, Le déterminant de la cohomologie, in Current trends in arithmetic algebraic geometry (ed. K. A. Ribet), Contemporary Mathematics, Volume 67, pp. 93-177 (American Mathematical Society, Providence, RI, 1985).

26. P. Deligne, J. F. Boutot, A. Grothendieck, L. Illusie and J. L. Verdier, Cohomologie étale, Lecture Notes in Mathematics, Volume 569 (Springer, 1977).

27. H. Esnault and E. Viehweg, Deligne-Beilinson cohomology, in Beilinson's conjectures on special values of L-functions (ed. M. Rapoport, N. Schappacher and P. Schneider), Perspectives in Mathematics, Volume 4, pp. 43-91 (Academic, 1988).

28. G. Faltings, Endlichkeitssätze für abelsche Varietäten über Zahlkörpern, Invent. Math. 73 (1983), 349-366.

29. G. Faltings, Calculus on arithmetic surfaces, Ann. Math. 119 (1984), 387-424.

30. G. Faltings, Finiteness theorems for abelian varieties over number fields, in Arithmetic geometry (ed. G. Cornell and J. H. Silverman), pp. 9-27 (Springer, 1986).

31. A. FuJIKI, Duality of mixed Hodge structures of algebraic varieties, Publ. RIMS Kyoto 16 (1980), 635-667.

32. D. FulEA, Höhere arithmetische $K$-Theorie, $\mathrm{PhD}$ thesis, University of Mannheim (1998).

33. W. Fulton, Intersection theory, Ergebnisse der Mathematik und ihrer Grenzgebiete, Volume 3 (Springer, 1984). 
34. H. Gillet, Riemann-Roch theorems for higher algebraic K-theory, Adv. Math. 40 (1981), 203-289.

35. H. Gillet And C. Soulé, Intersection theory using Adams operations, Invent. Math. 90 (1987), 243-277.

36. H. Gillet And C. Soulé, Arithmetic intersection theory, Publ. Math. IHES 72 (1990), 94-174.

37. H. Gillet and C. Soulé, Filtrations in higher algebraic $K$-theory, in Algebraic $K$ Theory, Seattle, WA, 1997, Proceedings of Symposia in Pure Mathematics, Volume 67, pp. 89-148 (American Mathematical Society, Providence, RI, 1999).

38. R. Godement, Théorie des faisceaux, Actualités Scientifiques et Industrielles, Volume 1252 (Hermann, Paris, 1964).

39. P. Griffiths And J. Harris, Principles of algebraic geometry (Wiley, 1994).

40. W. Gubler, Moving lemma for $K_{1}$-chains, J. Reine Angew. Math. 548 (2002), 1-19.

41. B. Harris AND B. WANG, Arithmetic height pairing of intersecting cycles, Int. Math. Res. Not. 4 (1993), 107-111.

42. R. HARTshorne, Algebraic geometry, Graduate Texts in Mathematics, Volume 52 (Springer, 1977).

43. M. Herrera AND D. Lieberman, Residues and principal values on complex spaces, Math. Ann. 194 (1971), 259-294.

44. H. HiRonakA, Resolution of singularities of an algebraic variety over a field of characteristic zero, Ann. Math. 79 (1964), 109-326.

45. J. Hu, Specialization of Green forms and arithmetic intersection theory, Arakelov Geometry Preprint Archive (available at www.institut.math.jussieu.fr/Arakelov) (2002).

46. A. Huber And J. Wildeshaus, Classical motivic polylogarithm according to Beilinson and Deligne, Documenta Math. 3 (1998), 27-133.

47. B. Iversen, Cohomology of sheaves, Universitext, Number 31 (Springer, 1986).

48. U. Jannsen, Deligne homology, Hodge- $D$-conjecture and motives, in Beilinson's conjectures on special values of L-functions (ed. M. Rapoport, N. Schappacher and P. Schneider), Perspectives in Mathematics, Volume 4, pp. 305-372 (Academic, 1988).

49. J. F. Jardine, Simplicial presheaves, J. Pure Appl. Alg. 47 (1987), 35-87.

50. J. F. Jardine, Generalized étale cohomology theories, Progress in Mathematics, Volume 146 (Birkhäuser, 1997).

51. J. R. KING, Log complexes of currents and functorial properties of the Abel-Jacobi map, Duke Math. J. 50 (1983), 1-53.

52. K. KöHLER, A Hirzebruch proportionality principle in Arakelov geometry, in Number fields and function fields-two parallel worlds, pp. 237-268, Progress in Mathematics, Volume 239 (Birkhäuser, 2005).

53. J. Kramer, An arithmetic theory of Siegel-Jacobi forms, Habilitationsschrift, ETH Zürich (1992).

54. Ch. Kratzer, $\lambda$-structure en $K$-théorie algébrique, Comment. Math. Helv. 55 (1980), 233-254.

55. S. S. Kudla, Derivatives of Eisenstein series and generating functions for arithmetic cycles, Séminaire Bourbaki 1999/2000, Exposés 865-879, Astérisque, Volume 276, pp. 341368 (Société Mathématique de France, Paris, 2002).

56. S. S. Kudla, Special cycles and derivatives of Eisenstein series, in Heegner points and Rankin L-series, pp. 243-270, Mathematical Sciences Research Institute Publications, Volume 49 (Cambridge University Press, 2004).

57. U. KüHN, Generalized arithmetic intersection numbers, C. R. Acad. Sci. Paris Sér. I 327 (1998), 283-288.

58. U. KüHN, Generalized arithmetic intersection numbers, J. Reine Angew. Math. 534 (2001), 209-236. 
59. J.-L. LodAy, $K$-théorie algébrique et représentations de groupes, Annls Scient. Éc. Norm. Sup. 9 (1976), 309-377.

60. V. Maillot, Géométrie d'Arakelov des variétés toriques et fibrés en droites intégrables, Mémoires de la Société Mathématique de France, Volume 80 (Société Mathématique de France, Paris, 2000).

61. V. Maillot and D. Roessler, Conjectures sur les dérivées logarithmiques des fonctions $L$ d'Artin aux entiers négatifs, Math. Res. Lett. 9 (2002), 715-724.

62. B. Malgrange, Ideals of differentiable functions (Oxford University Press, 1966).

63. A. MORIWAKI, Intersection pairing for arithmetic cycles with degenerate Green currents, E-print AG/9803054.

64. D. Mumford, Hirzebruch's proportionality theorem in the non-compact case, Invent. Math. 42 (1977), 239-272.

65. A. Ogus, The formal Hodge filtration, Invent. Math. 31 (1976), 193-228.

66. J. P. POLY, Sur l'homologie des courants à support dans un ensemble semi-analytique, Mémoires de la Société Mathématique de France, Volume 38, pp. 35-43 (Société Mathématique de France, Paris, 1974).

67. D. Quillen, Homotopical algebra, Lecture Notes in Mathematics, Volume 43 (Springer, 1967).

68. D. Quillen, Higher algebraic K-theory, I, in Algebraic K-theory I (ed. H. Bass), Lecture Notes in Mathematics, Volume 341, pp. 85-147 (Springer, 1973).

69. J. H. Silverman, The theory of height functions, in Arithmetic geometry (ed. G. Cornell and J. H. Silverman), pp. 151-166 (Springer, 1986).

70. C. Soulé, Opérations en $K$-théorie algébrique, Can. J. Math. 37 (1985), 488-550.

71. C. Soulé, D. Abramovich, J.-F. Burnol And J. Kramer, Lectures on Arakelov geometry, Cambridge Studies in Advanced Mathematics, Volume 33 (Cambridge University Press, 1992).

72. V. SRINIVAS, Algebraic K-theory, Progress in Mathematics, Volume 90 (Birkhäuser, 1991).

73. J. C. Tougeron, Idéaux de fonctions différentiables (Springer, 1972).

74. P. Vojta, Siegel's theorem in the compact case, Ann. Math. 133 (1991), 509-548.

75. S. Zhang, Small points and adelic metrics, J. Alg. Geom. 4 (1995), 281-300. 